F. F. Mende, A. S. Dubrovin

\title{
ALTERNATIVE IDEOLOGY OF ELECTRODYNAMICS
}

Moscow - 2016 
PACS 11.10.-Z

\section{Mende F. F., Dubrovin A. S.}

Alternative ideology of electrodynamics. Monograph. M.: Пepo, 2016. - 216 p., Fig. 62, ref. 78.

The role of the vector potential of magnetic field in the equations of induction is refined. The concept of the vector potential of electric field is introduced. It is shown what concept is, as the kinetic inductance of charges plays in the electrodynamics not less important role, than dielectric and magnetic constant. Is developed the mathematical model of the dispersion of electromagnetic waves in the conductors and the dielectrics with the use of a physical quantity of dielectric constant, which does not depend on frequency. It is shown that in the confined plasma the transverse plasma resonance can exist. They are introduced the concept of kinetic capacity. Provides a new way to display the wave equation. The way of writing of the equations of induction with the use by the substantional derivative obtained their symmetrical form. Is introduced the scalar-vector potential, in which the scalar potential of charge and its field they depend on speed.. Because of the laws of symmetric induction in the framework of the laws Galileo transformations obtained conversion fields in the transition from one inertial reference system to another, called Mende transformation. The introduction of this potential made it possible within the framework the Galileo conversions to explain the phase aberration and the transverse Doppler effect, and also power interaction of the current carrying systems without the use of a postulate about the Lorentz force. Are examined the bases of trans-coordinate electrodynamics in time-spatial giperkontinuum, in which it is improved the apparatus for differential calculus of field functions it is introduced the Dubrovin operator. The results of the experimental studies, which showed, are given that the charge is not the invariant of speed. Received in return the law of conservation of 4-pulse of the new law of conservation of angular balance.

For the specialists in the region of electrodynamics, radio physics, electronics, radio engineering, technical protection of information, theoretical and mathematical physics, and also students and graduate students of the corresponding specialties.

\section{Reviewers}

A. A. Rukhadze - doctor of physical and mathematical sciences, professor, laureate of state prizes and prize of M. V. Lomonosov.

N. N. Gorobez - doctor of physical and mathematical sciences, professor, head of department of applied electrodynamics of Kharkov national university V. N. Karazin

\section{ISBN}




\section{INTRODUCTION}

Past century is marked by the most great crisis in physics, when for the change to a fundamental understanding of the physical sense of natural phenomena and technical processes arrived new scientific orientators. Physicist P. Dirac proclaimed mathematical beauty by sole criterion for the selection of the way of development in theoretical physics. But mathematician M. Atya, realizing risk to be that lulled by the elegance, which is been based on unsteady soil, warned that the subordination of physics to mathematics conceals danger, since can bring into the region of the fabrications, which personify the mathematical perfection, but too distant from the physical reality or even having with it nothing in common.

The special feature of contemporary physics is its comparatively high financing with the fact that the realization of transparent and effective state and public control of the appropriate financial flows runs into the formidable difficulties. The situation, when physicists control themselves, creates favorable circumstances for all possible abuses of the hypertrophied authorities. Especially complex state of affairs occurs in the sphere of basic physical research, which includes, in particular, the theoretical bases of electrodynamics. The extremely high level of the mathematization of scientific works in this sphere leads to the fact that even the highly skilled specialists of adjacent regions or altogether only belonging to different scientific schools begin to speak "in the different languages" and they cease to understand each other.

The scientific results of the individual scientists (such as Einstein and Khoking) proclaim immutable truth similarly to religious dogmas. But open and secret prohibitions to the critical analysis of the works of the acknowledged coryphaei are always disastrous for the scientific progress and unavoidably they lead to the stagnation. However, any criticism must be objective and design. At the basis of physics was always it must remain physical experiment, and the correspondence to physical experiment must always be the principal criterion of the truth of physical theories. The mathematical rigor of physical theories is also important, but is not less important that, so that the physical sense of phenomena and processes would not be hidden, obscured by mathematical formalizations. In this connection, is fruitful the adoption in the theoretical electrodynamics of methodological approaches from electrical engineering (in particular, the method of equivalent diagrams) in view of the fact that they make it possible to develop mathematical models with a comparatively simple, transparent physical sense. This "electrotechnical" approach to the electrodynamics is successfully realized in the number of the work of Mende F.F. Specifically, support on the physical experiment and the comprehensive disclosure of the physical sense of models they are assumed as the basis in this work alternative of the ideology of electrodynamics stated.

Finally, one additional brake of the development of science - its excessive popularization in the commercial interests. The science, chained into the shackles of the yellow press, when on the covers of popular periodicals for the larger psychological effect are depicted allegedly the brilliant persons with the limited physical possibilities, tendentiously praised by journalists, who do not absolutely examine sci- 
ence itself on themselves, causes bewilderment. The high mathematization of physical theories only helps yellow press to give to physics the halo of mysticity, taking away the reader from the truth. Is preferable the qualified popularization of physics by scientists themselves, but furthermore, it must rest on the objective information about the results of physical experiments and the comprehensive disclosure of the physical sense of theoretical models.

All this gave birth to the most severe crisis in contemporary physics. But this state of affairs cannot continue eternally. Now situation in physics greatly resembles that, which preceded the fall of the system of Ptolemy. For the change to decrepit dogmas prepare to arrive new progressive ideas and views. So that it is better to understand, what renovation contemporary physics requires, necessary critically to analyze as why arose this deep and prolonged crisis.

Passage from comparatively simple and intuitively intelligible classical ideas about the space and time to the relativistic was critical moment. But after connecting relativity with the quantization of action, complete geometrization of gravity and propagation of the principle of geometrization on other physical interactions the imperfection of the prevailing ideas and views became obvious. The root of this imperfection consists in the fundamental disagreement between physics and mathematics, when the mathematical apparatus for physics increasingly more degenerates into polumisticheskuyu scholasticism, within the framework by which the objective physical sense of phenomena increasingly more slips off, and the role of the subjective consciousness of observer and unknown "magic" force of abstract mathematical formulas becomes of ever of more fundamental. One should recognize that the noted disagreement of physics and mathematics began to increase long before the victorious procession of the theory of relativity. Probably, by historically first especially "solid nut" for mathematical physics proved to be electrodynamics. Passage from the mechanics of material points and their final totalities to the formal description of continuous in the space and the time of electromagnetic field required the attraction of more powerful mathematical apparatus, but the development of mathematics, which goes in many respects according to its own internal laws, it did not chronically answer the demands of vigorously developing physics.

In the initial form the system of equations of classical electrodynamics was recorded by Maxwell in his famous treatise [1] with the use of quaternion calculation within the framework of classical ideas about the space and time, that allow the conversions of Galileo upon transfer from the examination of electromagnetic field in one inertial reference system to the examination of the same field in another inertial reference system. However, it was immediately explained that the apparatus for quaternion calculation in mathematics was developed not so well so that physics they could it successfully apply to the wide circle of the tasks of electrodynamics. In order to draw into the electrodynamics the simpler and more effective means of mathematical physicists, Hertz and Heaviside reformulated Maxwell's equations from the language of quaternion calculation to the language of vector analysis. 
At that time it seemed that the formulation of Hertz-Heaviside is equivalent to the initial formulation of Maxwell, but now already it is possible to establish that the equations, obtained by Hertz and Heaviside, are essential simplification in Maxwell's equations in the quaternions, moreover this simplification relates not only to their mathematical form, but also (that most important!) to their physical content, since in this case equations were deprived naturally Galileo- invariance of inherent in them. Nevertheless for the concretely undertaken inertial reference system (but not their totality) the equivalence of formulations occurred, by virtue of which the formulation of Hertz-Heaviside it obtained the deserved acknowledgement of scientific association it extruded in the theoretical and applied research the formulation of Maxwell himself.

The comprehensive analysis of Hertz-Heaviside equations by many scientists led to the development by Lorenz and Poincare the bases of the mathematical apparatus of the special theory of relativity. This was major step forward in comparison with the nonrelativistic theory of electromagnetic field, since it was possible to reveal the dependence of electromagnetic field on the relative speed of observer. But those leading of physics and mathematics of their time could not propose to the clear physical interpretation of their formulas. This is what writes in regard to this well-known specialist in the region of tensor analysis Rachewsky [2]: "The theory of relativity arose as a result the prolonged accumulation of the experimental material, which led to the deep conversion of our physical ideas about the forms of material and motion. And other physical quantities to the newly open experimental facts it was revealed after the whole series of the attempts to adapt previous ideas about the space, time that for these purposes it is necessary to reconstruct all these concepts radically. This task was executed in basic a. By Einstein in 1905. (special theory of relativity) and in 1915. (general theory of relativity). In other the task was executed was only in the sense that given the ordered formal mathematical description of new state of affairs. The task of the deep, really physical substantiation of this mathematical diagram still stands before physics".

At this determining moment physics proved to be on crossroads. One of the ways lay at the direction of further searches for the suitable mathematical apparatus for electrodynamics (to what, judging by everything, were inclined they themselves Lorenz and Poincare), but the physicist following Einstein it was banal along another way, who consisted in the decisive and uncompromising failure of the classical ideas about the space and the time with the passage to the relativistic ideas. Subsequently Logunov A.A. it convincingly showed that essence SR consists of the identification of the natural geometry of the electromagnetic field, described by Maxwell's equations in the formulation of Hertz-Heaviside, with the geometry of world physical space-time.

By the way of introduction into physics of known postulates, the theory of relativity in Einstein's version explained several important experimental results and in connection with this was obtained the acknowledgement of the wide circles of physicists. Relativistic ideology supported such those leading of mathematics of that time as Minkowski, Gilbert and Born. The principle of geometrization, which reflects secret dreams and expectations of many thinkers, was and remains espe- 
cially attractive for the mathematicians in this ideology, beginning from the idealistic views of great Ancient Greek philosopher Plato, to reduce all fundamental laws of universe to the geometric properties of the certain idealized mathematical objects.

Thus, mathematics, after yielding to temptation to subordinate to itself physics by means of the principle of geometrization so desired for it, proved to be unable to rise higher than the geometric means of thinking and it is worthy to satisfy the increasing needs of physics. Physics used that apparatus, which mathematics was ready to propose to it, and this unavoidably conducted to the creation of special, and then general theory of relativity and, further, to all to the increasing celebration of the principle of geometrization.

In accordance with them, the dependence of electromagnetic field on the speed of the motion of observer is not caused by the fundamental factors of physical nature of field itself, but it is defined by example through the dependence on it of the intervals of time and spatial distance (conversion of Lorenz) under the assumption of the relativistic invariance of electric charge. However, specialists (first of all, by experimenters) discovered, that the classical electrodynamics and SR, in spite of already the more centenary myth, are located in the contradiction to each other. However, contemporary experiences on the measurement of the speed of light in one direction (but the not averaged speed "back and forth" as, for example, in Fizeau's experiments and to them analogous) [3] contradict postulate SR about the constancy of the speed of light and is brought into question the physical validity of the conversions of Lorenz.

If we nevertheless adhere to ourselves the point of view of the fundamental direct dependence of field on the speed, then this dependence must be extended even to the absolute value of electric charge. Until recently this not invariance of charge was confirmed only by indirect empirical data, which were being consisted in the appearance of an electric potential on the superconductive windings and the tori during the introduction in them of direct current, or in the observation of the electric pulse of nuclear explosions. However, 2015 year was marked by the already direct experimental confirmation of this phenomenon as a result of detection and study of the pulse of the electric field, which appears with the warming-up of the plasma as a result of the discharge through the dischargers of the capacitors of great capacity. It turned out that in the process of the warming-up of plasma with an equal quantity in it of electrons and positive ions in it the unitary negative charge of free electrons, not compensated by slower positive ions, is formed. This fact contradicts not only the classical, but also relativistic conversions of electromagnetic field upon transfer from one ISO to another, testifying about the imperfection not only of classical, but also relativistic ideas about the space and the time.

Idea about the fact that the promising electrodynamics must assume existence of the dependence of electromagnetic field on the speed of the motion of observer, caused not by the geometry of space-time, and by physical nature of field, which does not assume the invariance of electric charge, was developed in a number of the work of Mende F.F. In these works, in is given the substantiation of introduc- 
tion into the electrodynamics instead of the classical and relativistic new conversions of electromagnetic field, which was called the conversions of Mende.

However, the sequential development of this radical idea, as not the invariance of charge, requires the deep revision of the mathematical apparatus for electrodynamics, called to the creation of the mathematical means of the more adequate description of passage from one ISO to another. This approach to the development of the mathematical apparatus for electrodynamics was proposed by Dubrovin A.S. This approach lies within the framework the sequential revision of ideas about the space and the time with the failure of the relativistic and the passage to the new ideas, which we call giperkontinualnymi and consider it their kind neoclassical in the sense that they they return fundamental role in the physicist to the conversions of Galileo. At the same time they allow the great possibilities of the invariance of various physical processes relative to various groups of the pseudo-orthogonal transformations of coordinates, which realize hyperbolic turnings with the invariance of the pseudo-Euclidean certificate of effective (by suitable means of that imagined) space-time. But the natural geometry of physical field does not identify in this case with the geometry of world physical space-time.

The concept of time-spatial giperkontinuuma is introduced as a result the joint study of the algebraic and geometric structures of the commutative algebras with one, elements of which are the functions of sine waves. The hypothesis of giperkontinuuma (about the hierarchical giperkontinualnoy structure of world physical space-time) serves as starting point of scientific studies in the direction of the generalization of ideas about the structure of space and time in the course of passage from the contemporary quantum scientific paradigm to the new system, that simultaneously structurally connecting up its framework continuity and discretion, dynamicity and static character, and also globality and locality. The hierarchical quality of giperkontinuuma limits the applicability of the conventional principle of geometrization in physics and the connected with it ideas of symmetry in the geometry due to the introduction into physics of the ideas of hierarchical quality, whose effectiveness have approved we with the creation of the standard model of the protected automated system (EMZAS) and the mathematical apparatus of EMZASnetworks.

So what does not be sufficient in the apparatus for contemporary mathematical physics? We consider that the contemporary mathematical analysis of field functions operates with differential calculus with the inadmissibly limited possibilities. The derivatives of field functions and the differential equations of field always relate to the concrete frame of reference. The corresponding conversions of differential equations are produced upon transfer from one frame of reference to another. If they preserve their form, then they speak about their invariance. But the vehicle for differential calculus of field functions prevailing at the present time does not assume the examination of the differential equations of field, which mutually connect two different frame of references. Specifically, this does not make it possible to adequately describe passage from one frame of reference to another with the examination of electromagnetic field. And precisely this makes urgent the development of new differential calculus, which we call trans-coordinate. This approach re- 
quires the reformation of the equations of electrodynamics to the new idea in comparison with the idea of Hertz-Heaviside, which we also call trans-coordinate (for greater detail, see in $\S 17)$. Trans-coordinate differential calculus and formulation of electrodynamics were developed by Dubrovin A.S.

Traditionally the development of mathematical analysis and mathematical physics was located under the effect of the geometry and the special geometric viewpoint connected with it, that also led, in the final analysis, to the victorious procession of the principle of geometrization in physics. Now situation changes radically. The development of mathematics increasingly more influences the information theory, which is developed in a stormiest, swiftest manner on the wave of revolutionary computerization and informatizatsii of society. Contemporary information theory uses a completely high level of abstraction in the idea of information, in comparison with which geometric viewpoint proves to be primitive. The development of giperkontinualnykh ideas about the space and the time together with the ideas of trans-coordinateawn they lie at the course of precisely this trend in development of physico-mathematical sciences under the effect of the ideas, borrowed from the information theory.

In this monograph it is shown that following the failure of the formulation of Hertz- Heaviside of Maxwell's equations it is necessary to generally forego Maxwell's equations, as such, together with the concept of electromagnetic field lying at their basis. Is exponential quotation from [3]: "in what does consist the basic initial reason for the discrepancy of the built by Maxwell electrodynamics? For the single-valued answer to this question... it should be noted that even in its time of amperes, Grossman, Gauss, Lentz, Neumann, Veber, Riemann and other they stood on the point of view, that, without being turned to the concept "of magnetic field", any magnetic interactions can be reduced to usual interactions of current elements or moving charges... in the electrodynamics repossessed then the point of view of Faraday and Maxwell, that the electrical and "magnetic" fields are the independent physical essences, although connected together. In the prevailing then historical situation given, erroneous from a physical point of view, assumptions predetermined by themselves entire further motion of the development of electrodynamics with the deliberately placed into it insoluble contradictions and the paradoxes". And further there: "for the noncontradictory reflection of the physical essence of the laws of electromagnetism necessary to completely forego any concepts "magnetic field" as certain independent physical essence... for determining the forces of interaction of moving in the physical vacuum of real space electric charges completely sufficient to consider the deformation of electrical pour on these charges, caused by the trivial effects of the being late potentials... To there remains only be surprised at the sagacity of the ampere, which warned that if we in the electrodynamics do not forego ourselves the concept "magnet", then subsequently this threatens by incredible confusion in the theory".

In the monograph are given the results of the experiments, which confirm the concept of the dependence of electric charge on the speed, and it is represented the extensive material, which is concerned the most important questions of classical electrodynamics, in particular: the conversion of electromagnetic field upon trans- 
fer from one IRS to of another, the phenomenon phase aberration, the transverse Doppler effect, power interaction of the current carrying systems, dipole emission. In this case were not used not only the postulates of the theory of relativity, but also postulate about the Lorentz force.

The dependence of electric charge on the speed must not be introduced as the sequential physical postulate, since it occurs the direct consequence Dubrovin A.S. proposed. the new dynamic law of conservation - of the law of conservation of kinetic balance. It is similar to the laws of the conservation of energy, pulse and moment of momentum, it follows from the variation least-action principle under the assumption of the general properties of space and time (this law it answers the property of the isotropy of giperkontinuum), substituting the law of conservation of 4-momentom of the theory of relativity, which reflects the property of the Lorentz invariance of the certificate of space-time continuum. But the role of this law is by no means limited only to this. The replacement of the law of conservation of 4momentom to the law of conservation of kinetic balance requires radical reconstruction of mechanics. In particular, proves to be possible mechanical motion with the faster-than-light speed, is revealed the essential specific character of rotary motion. Similarly, as the relativistic ideology of electrodynamics it led to the construction of relativistic physics, the alternative ideology of electrodynamics proposed in the monograph pretends to the role of the initiator of the process of creating new, giperkontinual physics. 


\section{PART I \\ REFINEMENT OF THE CONCEPTS OF THE CLASSICAL ELECTRODYNAMICS}

\section{CHAPTER 1 \\ THE LAWS OF INDUCTION AND THEIR ROLE IN THE CLASSICAL ELECTRODYNAMICS}

\section{§ 1. Maxwell equations and Lorentz force}

The laws of classical electrodynamics are phenomenological and reflect the collection of experimental facts and their physical nature is not known to us unfortunately, contemporary classical electrodynamics is not deprived of the contradictions, which did not up to now obtain their explanation. In order to understand these contradictions, and to also understand those purposes and tasks, which are placed in this work, let us briefly describe the existing situation.

The fundamental equations of contemporary classical electrodynamics are Maxwell equations [4]. They are written as follows for the vacuum:

$$
\begin{aligned}
\operatorname{rot} \mathbf{E} & =-\frac{\partial \mathbf{B}}{\partial t}, \\
\operatorname{rot} \mathbf{H} & =\frac{\partial \mathbf{D}}{\partial t}, \\
\operatorname{div} \mathbf{D} & =0, \\
\operatorname{div} \mathbf{B} & =0,
\end{aligned}
$$

where $\mathbf{E}, \mathbf{H}$ - tension of electrical and magnetic field, $\mathbf{D}=\varepsilon_{0} \mathbf{E}, \mathbf{B}=\mu_{0} \mathbf{H}$ - electrical and magnetic induction, $\mu_{0}, \varepsilon_{0}$ - magnetic and dielectric constant of vacuum. From these equations follow wave equations for the electrical and magnetic fields on

$$
\begin{aligned}
& \nabla^{2} \mathbf{E}=\mu_{0} \varepsilon_{0} \frac{\partial^{2} \mathbf{E}}{\partial t^{2}}, \\
& \nabla^{2} \mathbf{H}=\mu_{0} \varepsilon_{0} \frac{\partial^{2} \mathbf{H}}{\partial t^{2}} .
\end{aligned}
$$

These equations show that in the vacuum can be extended the plane electromagnetic waves, the velocity of propagation of which is equal to the speed of light

$$
c=\frac{1}{\sqrt{\mu_{0} \varepsilon_{0}}} .
$$

For the material media of Maxwell equations they take the following form:

$$
\operatorname{rot} \mathbf{E}=-\tilde{\mu} \mu_{0} \frac{\partial \mathbf{H}}{\partial t}=-\frac{\partial \mathbf{B}}{\partial t},
$$




$$
\begin{gathered}
r o t \mathbf{H}=n e \mathbf{v}+\tilde{\varepsilon} \varepsilon_{0} \frac{\partial \mathbf{E}}{\partial t}=n e \mathbf{v}+\frac{\partial \mathbf{D}}{\partial t}, \\
\operatorname{div} \mathbf{D}=n e, \\
\operatorname{div} \mathbf{B}=0,
\end{gathered}
$$

where, $\tilde{\mu}, \tilde{\varepsilon}$ - the relative magnetic and dielectric constants of the medium and $n$, $e, \mathbf{v}$ - density, value and charge rate.

The equation (1.1)-(1.11) are written in the assigned inertial measuring system (IMS), and in them there are no rules of passage of one IMS to another. The given equations also assume that the properties of charge do not depend on their speed, since in first term of the right side of equation (1.9) as the charge its static value is taken. The given equations also assume that the current can flow as in the electrically neutral medium, where there is an equal quantity of charges of both signs, so also to represent the self-contained flow of the charged particles, moreover both situations are considered equivalent.

In Maxwell equations are not contained indication that is the reason for power interaction of the current carrying systems; therefore to be introduced the experimental postulate about the force, which acts on the moving charge in the magnetic field. This the so-called magnetic part of the Lorentz force [5]

$$
\mathbf{F}_{L}=e\left[\mathbf{v} \times \mu_{0} \mathbf{H}\right] .
$$

However, in this axiomatic is an essential deficiency. If force acts on the moving charge, then in accordance with third Newton law the reacting force, which balances the force, which acts on the charge, must occur and to us must be known the place of the application of this force. In this case the magnetic field comes out as a certain independent substance and comes out in the role of the mediator between the moving charges, and if we want to find the force of their interaction, then we must come running to the services of this mediator. In other words, we do not have law of direct action, which would give immediately answer to the presented question, passing the procedure examined, i.e., we cannot give answer to the question, where are located the forces, the compensating action of magnetic field to the charge.

The relationship (1.12) from the physical point sight causes bewilderment. The forces, which act on the body in the absence of losses, must be connected either with its acceleration, if it accomplishes forward motion, or with the centrifugal forces, if body accomplishes rotary motion. Finally, static forces appear when there is the gradient of the scalar potential of potential field, in which is located the body. But in relationship (1.12) there is nothing of this. Usual rectilinear motion causes the force, which is normal to the direction motion. What some new law of nature? To this question in the classical electrodynamics there is no answer also.

Is certain, magnetic field is one of the important concepts of contemporary electrodynamics. Its concept consists in the fact that around any moving charge appears the magnetic field (Ampere law), whose circulation is determined by the relationship

$$
\text { f] } \mathbf{H} d \mathbf{l}=I,
$$


where $I$ - conduction current.

Equation (1.9) is the consequence of relationship (1.13), if we to the conduction current add bias current. As is known, Maxwell for the first time introduced bias current.

Let us especially note that the introduction of the concept of magnetic field does not be founded upon any physical basis, but it is the statement of the collection of some experimental facts. Using this concept, it is possible with the aid of the specific mathematical procedures to obtain correct answer with the solution of practical problems. But, unfortunately, there are a number of the physical questions, during solution of which within the framework the concepts of magnetic field, are obtained paradoxical results. Here one of them.

Using relationships (1.12) and (1.13) not difficult to show that with the unidirectional parallel motion of two like charges, or flows of charges, between them must appear the additional attraction. However, if we pass into the inertial system, which moves together with the charges, then there magnetic field is absent, and there is no additional attraction. This paradox does not have an explanation.

The force with power interaction of material structures, along which flows the current, are applied not only to the moving charges, but to the lattice, but in the concept of magnetic field to this question there is no answer also, since. in equations (1.1)-(1.13) the presence of lattice is not considered. At the same time, with the flow of the current through the plasma its compression (the so-called pinch effect), occurs, in this case forces of compression act not only on the moving electrons, but also on the positively charged ions. And, again, the concept of magnetic field cannot explain this fact, since in this concept there are no forces, which can act on the ions of plasma.

As the fundamental law of induction in the electrodynamics is considered Faraday law, consequence of who is the first Maxwell equation. However, here are problems. It is considered Until now that the unipolar generator is an exception to the rule of flow.

Let us give the still one exception, to which thus far no one turned attention. Faraday law indicates that the magnetic flux when through some section changes, then in the outline, which surrounds this section, vortex electric field appears. And if conductor is this outline, then in it currents are induced. Thus, in accordance with the law of the induction of Faraday the necessary condition of the appearance of currents in this outline is a change in the magnetic flux through the area, included by outline. If we insert the conducting outline into the magnetic field, then for the appearance of current in accordance with the Faraday law, the lines of force of magnetic field must intersect outline it. But it is known that the magnetic lines of force do not penetrate the superconductor and therefore they cannot intersect it. Therefore, if we take the superconductive ring, then magnetic flux through its section will be always equal to zero and as long as superconductor is superconductor, it cannot under any circumstances change.

Let us introduce the superconductive ring into the magnetic field. Naturally so that the magnetic flux through the section of ring would remain zero, it is necessary to compensate for external magnetic field in such a way that the magnetic flux 
through the section of ring would not change. This can be made an only method, after exciting in the ring the persistent currents, whose magnetic fields compensate for external magnetic field. But in order to excite such currents, it is necessary to the wire of the superconductive ring to apply electric field. But arises question, as such fields can arise, if summary magnetic flux through the section of ring did not change, and in accordance with the Faraday law vortex electrical fields on there must not be.

All these examples be evidence the fact that the law of the induction of Faraday is inaccurate or not complete and does not reflect all possible versions of the appearance of electrical fields on with a change of the magnetic field or during the motion in the Ger.

Let us give one additional statement of the work [4]: "The observations of Faraday led to the discovery of new law about the connection of electrical and magnetic fields on: in the field, where magnetic field changes in the course of time, is generated electric field". But from this law also there is an exception. Actually, the magnetic fields be absent out of the long solenoid; however, electric fields are generated with a change of the current in this solenoid around the solenoid. Is explained this fact thereby that around the long solenoid there is a circulation of vector potential [4]. When the flow of the magnetic induction of solenoid changes, then a circulation control of vector potential appears. With this interpretation of this phenomenon these changes lead to the appearance of electrical fields on out of the solenoid. In the work [4] even it is indicated that into 1956. Bohm and Aron experimentally detected this potential. But the point of view about existence of vector potential out of the long solenoid, where magnetic fields are absent, also runs into a number of the fundamental difficulties, which we will discuss with the examination of the law of the induction of Faraday.

In the classical electrodynamics does not find its explanation this wellknown physical phenomenon, as phase aberration of light, when with the observation of stars from moving IMS, telescope must be inclined to a certain angle in the direction of motion.

From entire aforesaid it is possible to conclude that in the classical electrodynamics there is number of the problems, which still await their solution. But, before passing to the solution of these problems and outlining the methods of their solution, let us trace that way, which is past the classical electrodynamics from the day of its base to the present.

\section{$\S 2$. Laws of the magnetoelectric induction}

The important task of electrodynamics is the presence of laws governing the appearance of electrical fields on, and, therefore, also the forces of those acting on the charge, at the particular point spaces, since only electric fields, generated other one or method or another, exert power influences on the charge. Such fields can be obtained, changing the arrangement of other charges around this point of space or accelerating these charges. If around the point in question is some static configuration of charges, then the tension of electric field will be at the particular point de- 
termined by the relationship $\mathbf{E}=-\operatorname{grad} \varphi$, where $\varphi$ is scalar potential at the assigned point, determined by the assigned configuration of charges. If we change the arrangement of charges, then this new configuration will correspond other values of scalar potential, and, therefore, also other values of the tension of electric field. But, making this, it is necessary to move charges in the space, and this displacement in the required order is combined with their acceleration and subsequent retarding. Acceleration or retarding of charges also can lead to the appearance in the surrounding space of induction electrical fields on. Can arise another stationary situation, when after their acceleration charges move in the environment of the considered point with the constant velocity along circular or other locked trajectories. In this case due to the presence of the three-dimensional velocity gradients in the flows of the moving charges configurative electric fields can appear.

In the electrodynamics the fundamental law of induction is Faraday law [6, 7]. In the contemporary electrodynamics it is written as follows:

$$
\text { ff } \mathbf{E} d \vec{l}=-\frac{\partial \Phi_{B}}{\partial t}=-\mu \int \frac{\partial \mathbf{H}}{\partial t} d \mathbf{s}=-\int \frac{\partial \mathbf{B}}{\partial t} d \mathbf{s},
$$

where $\mathbf{B}=\mu \mathbf{H}$ - magnetic induction vector, $\Phi_{B}=\mu \int \mathbf{H} d \mathbf{s}$ - flow of magnetic induction, and $\mu=\tilde{\mu} \mu_{0}$ - magnetic permeability of medium. It follows from this law that the circulation integral of the vector of electric field is equal to a change in the flow of magnetic induction through the area, which this outline covers. It is immediately necessary to emphasize the circumstance that the law in question presents the processes of mutual induction, since. for obtaining the circulation integral of the vector $\mathbf{E}$ we take the strange magnetic field, formed by strange source. From relationship (2.1) obtain the Maxwell first equation

$$
\operatorname{rot} \mathbf{E}=-\frac{\partial \mathbf{B}}{\partial t} .
$$

Let us immediately point out to the terminological error. Faraday law should be called not the law of electromagnetic, as is customary in the existing literature, but by the law of magneto electric induction, since. a change in the magnetic fields on it leads to the appearance of electrical fields on, but not vice versa.

Let us introduce the vector potential of the magnetic field $\mathbf{A}_{H}$, which satisfies the equality

$$
\mu \llbracket \mathbf{A}_{H} d \mathbf{l}=\Phi_{B},
$$

where the outline of the integration coincides with the outline of integration in relationship (2.1), and the vector $\mathbf{A}_{H}$ is determined in all sections of this outline, then

$$
\mathbf{E}=-\mu \frac{\partial \mathbf{A}_{H}}{\partial t} .
$$

Introduced thus vector $\mathbf{A}_{H}$ determines the local connection between it and by electric field, and also between the gradients this vector and the magnetic field. It is not difficult to show that introduced thus vector $\mathbf{A}_{H}$, is connected with the magnetic field with the following relationship:

$$
\operatorname{rot} \mathbf{A}_{H}=\mathbf{H} \text {. }
$$


Thus, if is determined vector $\mathbf{A}_{H}$, its local time derivative at any point of space, and also its gradients, then there is a possibility to determine immediately both vector $\mathbf{E}$, and vector $\mathbf{H}$.

The vector potential of magnetic field can be directly obtained also from the Ampere law, which was known long before Maxwell equations. The Ampere law, expressed in the vector form, determines magnetic field at the point $x, y, z$

$$
\mathbf{H}=\frac{1}{4 \pi} \int \frac{I d \mathbf{l} \times \mathbf{r}}{r^{3}},
$$

where $I$ - current in the element $d \mathbf{l}, \mathbf{r}$ - vector, directed from $d \mathbf{l}$ to the point $x, y, z$.

It is possible to show that

$$
\frac{[\mathbf{r} d \mathbf{l}]}{r^{3}}=\operatorname{grad}\left(\frac{1}{r}\right) \times d \mathbf{l}
$$

and besides the fact that

$$
\operatorname{grad}\left(\frac{1}{r}\right) \times d \mathbf{l}=\operatorname{rot}\left(\frac{d \mathbf{l}}{r}\right)-\frac{1}{r} \operatorname{rot} d \mathbf{l} .
$$

But the rotor $d \mathbf{l}$ is equal to zero and therefore is final

$$
\mathbf{H}=\operatorname{rot} \int\left(\frac{d \mathbf{l}}{4 \pi r}\right)=\operatorname{rot} \mathbf{A}_{H},
$$

where

$$
\mathbf{A}_{H}=\int I\left(\frac{d \mathbf{l}}{4 \pi r}\right) .
$$

In this case vector potential is determined no longer through the magnetic flux, but through the current, which flows through the specific section of conductor. the remarkable property of this expression is that that the vector potential depends from the distance to the observation point as . Specifically, this property makes it possible to obtain emission laws.

Since $I=g v$, where $g$ the quantity of charges, which falls per unit of the length of conductor, we obtain:

$$
\mathbf{A}_{H}=\int \frac{g v d \mathbf{l}}{4 \pi r} .
$$

For the single charge $e$ this relationship takes the form:

$$
\mathbf{A}_{H}=\frac{e \mathbf{v}}{4 \pi r},
$$

and since is fulfilled relationship (2.3), that

$$
\mathbf{E}=-\mu \int \frac{g \frac{\partial v}{\partial t} d \mathbf{l}}{4 \pi r}=-\mu \int \frac{g a d \mathbf{l}}{4 \pi r},
$$

where $a$ - acceleration of charge.

This relationship appears as follows for the single charge:

$$
\mathbf{E}=-\frac{\mu e \mathbf{a}}{4 \pi r} \text {. }
$$

This is the law of induction, which connects the appearance of electrical fields on directly with the acceleration of charge. 
If the discussion deals with the motion in the field of the three-dimensional changing vector potential, then for finding the induced electrical fields on should be used total derivative.

$$
\mathbf{E}^{\prime}=-\mu \frac{d \mathbf{A}_{H}}{d t}
$$

The prime near the vector $\mathbf{E}$ means that we determine this field in the moving coordinate system. This means that the vector potential can have not only local, but also convection derivative, i.e., it can change both due to the change in the time and due to the motion in the three-dimensional changing field of this potential. The relationship (2.5) can be rewritten as follows:

$$
\mathbf{E}^{\prime}=-\mu \frac{\partial \mathbf{A}_{H}}{\partial t}-\mu(\mathbf{v} \nabla) \mathbf{A}_{H},
$$

where $\mathbf{v}$ - speed of the prime system. The convective part of the force, which acts on the charge in the moving system will be written down

$$
\mathbf{F}_{v, 1}^{\prime}=-\mu e(\mathbf{v} \nabla) \mathbf{A}_{H} .
$$

This force depends only on the gradients of vector potential and charge rate.

The charge, which moves in the field of the vector potential $\mathbf{A}_{H}$ with the speed $\mathbf{v}$, possesses potential energy [3]

$$
W=-e \mu\left(\mathbf{v} \mathbf{A}_{H}\right) \text {. }
$$

Therefore must exist one additional force, which acts on the charge in the moving coordinate system, namely:

$$
\mathbf{F}_{v, 2}^{\prime}=-\operatorname{grad} W=e \mu \operatorname{grad}\left(\mathbf{v} \mathbf{A}_{H}\right) .
$$

Thus, the value $\left(\mathbf{v} \mathbf{A}_{H}\right)$ plays the same role, as the scalar potential $\varphi$, whose gradient also gives force. Consequently, the composite force, which acts on the charge, which moves in the field of vector potential, can have three components and will be written down as

$$
\mathbf{F}^{\prime}=-e \mu \frac{\partial \mathbf{A}_{H}}{\partial t}-e \mu(\mathbf{v} \nabla) \mathbf{A}_{H}+e \mu \operatorname{grad}\left(\mathbf{v} \mathbf{A}_{H}\right) .
$$

The first of the components of this force acts on the fixed charge, when vector potential changes in the time and has local time derivative. Second component is connected with the motion of charge in the three-dimensional changing field of this potential. Entirely different nature in force, which is determined by last term of relationship (2.6). It is connected with the fact that the charge, which moves in the field of vector potential, it possesses potential energy, whose gradient gives force. From relationship (2.6) follows

$$
\mathbf{E}^{\prime}=-\mu \frac{\partial \mathbf{A}_{H}}{\partial t}-\mu(\mathbf{v} \nabla) \mathbf{A}_{H}+\mu \operatorname{grad}\left(\mathbf{v} \mathbf{A}_{H}\right)
$$

This is a complete law of mutual induction. It defines all electric fields, which can appear at the assigned point of space, this point can be both the fixed and that moving. This united law includes and Faraday law and that part of the Lorentz force, which is connected with the motion of charge in the magnetic field, and without any exceptions gives answer to all questions, which are concerned mutual 
magneto electric induction. It is significant, that, if we take rotor from both parts of equality (2.7), attempting to obtain the Maxwell first equation, then it will be immediately lost the essential part of the information, since. rotor from the gradient is identically equal to zero.

If we isolate those forces, which are connected with the motion of charge in the three-dimensional changing field of vector potential, and to consider that

$$
\mu \operatorname{grad}\left(\mathbf{v} \mathbf{A}_{H}\right)-\mu(\mathbf{v} \nabla) \mathbf{A}_{H}=\mu\left[\mathbf{v} \times \operatorname{rot} \mathbf{A}_{H}\right],
$$

that from (2.6) we will obtain

$$
\mathbf{F}_{v}^{\prime}=e \mu\left[\mathbf{v} \times \operatorname{rot} \mathbf{A}_{H}\right],
$$

and taking into account (2.4), let us write down

$$
\mathbf{F}_{v}^{\prime}=e \mu[\mathbf{v} \times \mathbf{H}] .
$$

This is a magnetic part of the Lorentz force.

Electrical regiment, that acts on the charge in this case will be written down

$$
\mathbf{E}_{v}^{\prime}=\mu[\mathbf{v} \times \mathbf{H}],
$$

and it is final

$$
\mathbf{F}^{\prime}=e \mathbf{E}+e \mathbf{E}_{v}^{\prime}=-e \frac{\partial \mathbf{A}_{H}}{\partial t}+e \mu[\mathbf{v} \times \mathbf{H}] .
$$

Can seem that relationship (2.11) presents Lorentz force; however, this not thus. In this relationship the field $\mathbf{E}$, and the field $\mathbf{E}_{v}^{\prime}$ are induction: the first is connected with a change of the vector potential with time, the second is obliged to the motion of charge in the three-dimensional changing field of this potential. In order to obtain the total force, which acts on the charge, necessary to the right side of relationship (2.11) to add the term $-e \operatorname{grad} \varphi$

$$
\mathbf{F}_{\Sigma}^{\prime}=-e \operatorname{grad} \varphi-e \frac{\partial \mathbf{A}_{H}}{\partial t}+e \mu[\mathbf{v} \times \mathbf{H}],
$$

where $\varphi$ - scalar potential at the observation point. In this case relationship (2.7) can be rewritten as follows:

$$
\mathbf{E}^{\prime}=-\mu \frac{\partial \mathbf{A}_{H}}{\partial t}-\mu(\mathbf{v} \nabla) \mathbf{A}_{H}+\mu \operatorname{grad}\left(\mathbf{v} \mathbf{A}_{H}\right)-\operatorname{grad} \varphi,
$$

or after writing down the first two members of the right side of relationship (2.12) as the derivative of vector potential on the time, and also, after introducing under the sign of gradient two last terms, we will obtain

$$
\mathbf{E}^{\prime}=-\mu \frac{d \mathbf{A}_{H}}{d t}+\operatorname{grad}(\mu(\mathbf{v A})-\varphi) .
$$

If both parts of relationship (2.12) are multiplied by the magnitude of the charge, then will come out the total force, which acts on the charge. From Lorentz force it will differ in terms of the force $-e \mu \frac{\partial \mathbf{A}_{H}}{\partial t}$. From relationship (2.13) it is evident that the value $\mu(\mathbf{v A})-\varphi$ plays the role of the generalized scalar potential. After taking rotor from both parts of relationship (2.13) and taking into account that rot $\operatorname{grad}=0$, we will obtain 


$$
\operatorname{rot} \mathbf{E}^{\prime}=-\mu \frac{d \mathbf{H}}{d t}
$$

If we in this relationship replace total derivative by the quotient, then we will obtain the Maxwell first equation.

This examination maximally explained the physical picture of mutual induction. We specially looked to this question from another point of view, in order to permit those contradictory judgments, which occur in the fundamental works according to the theory of electricity.

Previously Lorentz force was considered as the fundamental experimental postulate, not connected with the law of induction. By calculation to obtain last term of the right side of relationship (2.11) was only within the framework special relativity (SR), after introducing two postulates of this theory. In this case all terms of relationship (2.11) are obtained from the law of induction, using the Galileo conversions. Moreover relationship (2.11) this is a complete law of mutual induction, if it are written down in the terms of vector potential. And this is the very thing rule, which gives possibility, knowing fields in one IMS, to calculate fields in another.

The structure of the forces, which act on the moving charge, is easy to understand based on the example of the case, when the charge moves between two parallel planes, along which flows the current (Fig. 1). Let us select for the coordinate axis in such a way that the axis $z$ would be directed normal to planes, and the axis $y$ was parallel to planes.

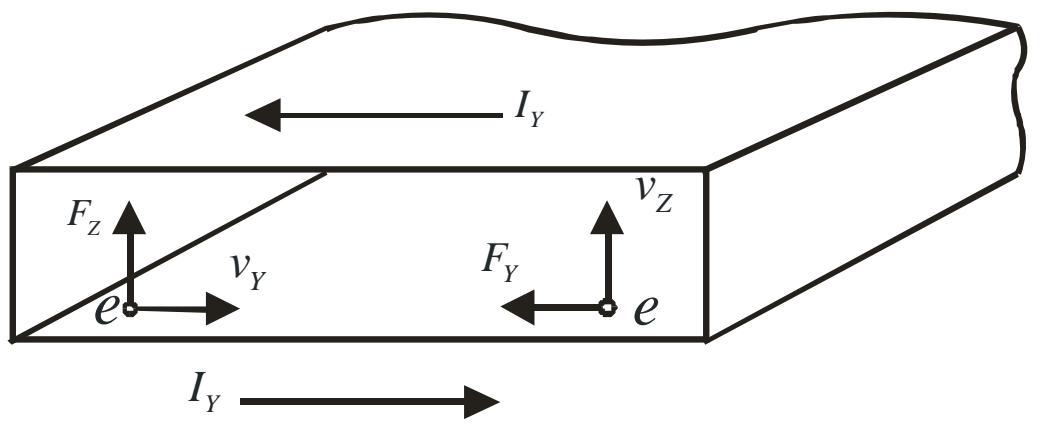

Fig. 1. Forces, which act on the charge, which moves in the field of vector potential

Then for the case, when the distance between the plates considerably less than their sizes (in this case on the picture this relationship not observed), the magnetic field $H_{x}$ between them will be equal to the specific current $I_{y}$, which flows along the plates. If consider, that the vector potential on the lower plate is equal to zero, then it's $y$ - the component, calculated off the lower plate, will grow according to the law

$$
A_{y}=I_{y} z
$$

If charge moves in the direction of the axis $y$ near the lower plate with the speed $v_{y}$, then the force $F_{z}$, which acts on the charge, is determined by last term of relationship (2.6) and it is equal 


$$
F_{z}=e \mu v_{y} I_{y}
$$

is directed this force from the lower plate toward the upper.

If charge moves along the axis $z$ from the lower plate to the upper with the speed $v_{z}=v_{y}$, then for finding the force should be used already second term of the right side of relationship (2.6). This force in the absolute value is again equal to the force, determined by relationship (2.14), and is directed to the side opposite to axis $y$. With any other directions of motion the composite force will be the vector sum of two forces, been last terms of relationship (2.6). However, the summary amount of this force will be determined by relationship (2.11), and this force will be always normal to the direction of the motion of charge. Earlier was considered the presence of this force as the action of the Lorentz force, whose nature was obscure, and it was introduced as experimental postulate. It is now understandable that it is the consequence of the combined action of two forces, different in their nature, whose physical sense is now clear.

Understanding the structure of forces gives to us the possibility to look to the already known phenomena from other side. With which is connected existence of the forces, which do extend loop with the current? In this case this circumstance can be interpreted not as the action of Lorentz force, but from an energy point of view. The current, which flows through the element of annular turn is located in the field of the vector potential, created by the remaining elements of this turn, and, therefore, it has it stored up potential energy. The force, which acts on this element, is caused by the presence of the potential gradient energy of this element and is proportional to the gradient to the scalar product of the current strength to the vector potential at the particular point. Thus, it is possible to explain the origin of ponder motive (mechanical) forces. If current broken into the separate current threads, then they all will separately create the field of vector potential. Summary field will act on each thread individually, and, in accordance with last term of the right side of relationship (2.6), this will lead to the mutual attraction. Both in the first and in the second case in accordance with the general principles system is approached the minimum of potential energy.

One should emphasize that in relationship (2.8) and (2.9) all fields have induction origin, and they are connected first with of the local derivative of vector potential, then by the motion of charge in the three-dimensional changing field of this potential. If fields in the time do not change, then in the right side of relationships (2.8) and (2.9) remain only last terms, and they explain the work of all existing electric generators with moving mechanical parts, including the work of unipolar generator. Relationship (2.7) gives the possibility to physically explain all composing tensions electric fields, which appears in the fixed and that moving the coordinate systems. In the case of unipolar generator in the formation of the force, which acts on the charge, two last addend right sides of equality (2.7) participate, introducing identical contributions.

With conducting of experiments Faraday established that in the outline is induced the current, when in the adjacent outline direct current is switched on or is 
turned off or adjacent outline with the direct current moves relative to the first outline. Therefore in general form Faraday law is written as follows:

$$
\text { ff } \mathbf{E}^{\prime} d \mathbf{l}^{\prime}=-\frac{d \Phi_{B}}{d t} \text {. }
$$

This writing of law indicates that with the determination of the circulation $\mathbf{E}$ in the moving prime coordinate system, near $\mathbf{E}$ and $d \mathbf{l}$ must stand primes and should be taken total derivative. But if circulation is determined in the fixed coordinate system, then primes near $\mathbf{E}$ and $d \mathbf{l}$ be absent, but in this case to the right in expression (2.15) must stand particular time derivative. Usually in the existing literature during the record the law of magneto electric induction in this fact attention they do not accentuate.

The complete time derivative in relationship (2.15) indicates the independence of the eventual result of appearance emf in the outline from the method of changing the flow. Flow can change both due to the change $\mathbf{B}$ with time and because the system, in which is measured the circulation $f \mathbf{E}^{\prime} d \mathbf{l}^{\prime}$, it moves in the threedimensional changing field $\mathbf{B}$. The value of magnetic flux in relationship (2.15) is given by the expression

$$
\Phi_{B}=\int \mathbf{B} d \mathbf{s}^{\prime},
$$

where the magnetic induction $\mathbf{B}=\mu \mathbf{H}$ is determined in the fixed coordinate system, and the element $d \mathbf{s}^{\prime}$ is determined in the moving system.

Taking into account (2.15), we obtain from (2.16)

$$
\text { ๆf } \mathbf{E}^{\prime} d \mathbf{l}^{\prime}=-\frac{d}{d t} \int \mathbf{B} d \mathbf{s}^{\prime}
$$

and further, since $\frac{d}{d t}=\frac{\partial}{\partial t}+\mathbf{v}$ grad, let us write down

$$
\text { โf } \mathbf{E} d \mathbf{l}^{\prime}=-\int \frac{\partial \mathbf{B}}{\partial t} d \mathbf{s}^{\prime}-\int[\mathbf{B} \times \mathbf{v}] d \mathbf{l}^{\prime}-\int \mathbf{v} \operatorname{div} \mathbf{B} d \mathbf{s}^{\prime} .
$$

In this case contour integral is taken on the outline $d l^{\prime}$, which covers the area $d \mathbf{s}^{\prime}$. Let us immediately note that entire following presentation will be conducted under the assumption the validity of the Galileo conversions, i.e., $d \mathbf{l}^{\prime}=d \mathbf{l}$ and $d \mathbf{s}^{\prime}=d \mathbf{s}$. From (2.17) follows the well-known result

$$
\mathbf{E}^{\prime}=\mathbf{E}+[\mathbf{v} \times \mathbf{B}],
$$

from which follows that during the motion in the magnetic field the additional electric field, determined by last term of relationship appears (2.18). Let us note that this relationship is obtained not by the introduction of postulate about the Lorentz force, or from the conversions of Lorenz, but directly from the Faraday law, moreover within the framework the Galileo conversions. Thus, Lorentz force is the direct consequence of the law of magneto electric induction.

The relationship follows from the Ampere law

$$
\mathbf{H}=\operatorname{rot} \mathbf{A}_{H} \text {. }
$$

Then fields on relationship (2.17) for those induced it is possible to rewrite

$$
\mathbf{E}^{\prime}=-\mu \frac{\partial \mathbf{A}_{H}}{\partial t}+\mu[\mathbf{v} \times \operatorname{rot} \mathbf{A}],
$$

and further 


$$
\mathbf{E}^{\prime}=-\mu \frac{\partial \mathbf{A}_{H}}{\partial t}-\mu(\mathbf{v} \nabla) \mathbf{A}_{H}+\mu \operatorname{grad}\left(\mathbf{v} \mathbf{A}_{H}\right) .
$$

Again came out relationship (2.7), but it is obtained directly from the Faraday law. True, and this way thus far not shedding light on physical nature of the origin of Lorentz force, since the true physical causes for appearance and magnetic field and vector potential to us nevertheless are not thus far clear.

With the examination of the forces, which act on the charge, we limited to the case, when the time lag, necessary for the passage of signal from the source, which generates vector potential, to the charge itself was considerably less than the period of current variations in the conductors. Now let us remove this limitation.

The Maxwell second equation in the terms of vector potential can be written down as follows:

$$
\operatorname{rot} \operatorname{rot} \mathbf{A}_{H}=\mathbf{j}\left(\mathbf{A}_{H}\right),
$$

where $\mathbf{j}\left(\mathbf{A}_{H}\right)$ - certain functional from $\mathbf{A}_{H}$, depending on the properties of the medium in question. If is carried out $\mathrm{Ohm}$ law $\mathbf{j}=\sigma \mathbf{E}$, then

$$
\left(\mathbf{j} \mathbf{A}_{H}\right)=-\sigma \mu \frac{\partial \mathbf{A}_{H}}{\partial t} \text {. }
$$

For the free space relationship (2.20) takes the form:

$$
\mathbf{j}\left(\mathbf{A}_{H}\right)=-\mu \varepsilon \frac{\partial^{2} \mathbf{A}_{H}}{\partial t^{2}} .
$$

For the free charges, which can move without the friction, functional will take the form

$$
\mathbf{j}\left(\mathbf{A}_{H}\right)=-\frac{\mu}{L_{k}} \mathbf{A}_{H},
$$

where $L_{k}=\frac{m}{n e^{2}}$ - kinetic inductance of charges [8]. In this relationship $m, e$ and $n$ - mass of charge, its value and density respectively.

The relationship (2.21)-(2.23) reflect well-known fact about existence of three forms of the electric current: active and two reactive. Each of them has characteristic dependence on the vector potential. This dependence determines the rules of the propagation of vector potential in different media. Here one should emphasize that the relationships (2.21)-(2.23) assume not only the presence of current, but also the presence of those material media, in which such currents can leak. The conduction current, determined by relationships (2.21) and (2.23), can the leak through the conductors, in which there are free current carriers. Permittance current, or bias current, can the leak through the free space or the dielectrics. For the free space relationship (2.20) takes the form:

$$
\operatorname{rot} \operatorname{rot} \mathbf{A}_{H}=-\mu \varepsilon \frac{\partial^{2} \mathbf{A}_{H}}{\partial t^{2}} .
$$

This wave equation, which attests to the fact that the vector potential can be extended in the free space in the form of plane waves, and it on its informativeness does not be inferior to the wave equations, obtained from Maxwell equations. 
From the aforesaid it follows that the writing of the law of induction in the terms of vector potential makes deeper physical sense than the writing of this law in the formulation of Faraday. Its use shedding light on many physical phenomena, which previously were not intelligible. Problem consists only in the fact that to us is not thus far clear physical nature of the vector potential of magnetic field. And, if it will be possible to explain physical nature of this potential, then is solved the very important problem both of theoretical and applied nature.

Let us examine the still one previously not known physical phenomenon [8]. Current density in the surface layer of superconductor has the following dependence [7]:

$$
j(z)=j_{0} e^{-\frac{z}{\lambda_{L}}}
$$

where $z$ - the coordinate, directed to the depth of superconductor and

$$
\lambda_{L}=\sqrt{\frac{m}{\mu_{0} n e^{2}}}
$$

is London depth of penetration.

Since $j(z)=n e v(z)$, we obtain charge rate

$$
v(z)=v_{0} e^{-\frac{z}{\lambda_{L}}}
$$

If at the assigned point of superconductor the speed of current carriers and vector potential is known, then, as follows from the formula (2.12), electric field it is determined by the relationship

$$
\mathbf{E}_{k}=\mu \operatorname{grad}\left(\mathbf{v} \mathbf{A}_{H}\right) .
$$

This field directed along the normal toward the surface of superconductor can be named electro kinetic field, since. it is connected with the motion of current carriers.

The magnetic field on its surface of superconductor, equal to specific current, can be determined from the relationship

$$
H_{0}=n e v_{0} \lambda_{L} \text {. }
$$

Using (2.23)-(2.27), we obtain the absolute value of the electro kinetic field:

$$
E_{k}=\frac{2 m H_{0}^{2}}{n^{2} e^{3} \lambda^{3}}
$$

With the zero of temperatures the critical magnetic field of niobium is equal $H_{0}=1.5 \times 10^{5} \mathrm{~A} / \mathrm{m}$, and $n=5.4 \times 10^{28} 1 / \mathrm{m}^{3}$. After substituting the values of these values in the relationship (2.28), we obtain $E_{k}=240 \mathrm{~V} / \mathrm{m}$.

\section{§ 3. Laws of the electromagnetic induction}

The Faraday law shows, how a change in the magnetic fields on it leads to the appearance of electrical fields on. However, does arise the question about that, it does bring a change in the electrical fields on to the appearance of any others fields on and, in particular, magnetic? Maxwell gave answer to this question, after intro- 
ducing bias current into its second equation. In the case of the absence of conduction currents the Maxwell second equation appears as follows:

where $\mathbf{D}=\varepsilon \mathbf{E}$ - electrical induction.

$$
\operatorname{rot} \mathbf{H}=\varepsilon \frac{\partial \mathbf{E}}{\partial t}=\frac{\partial \mathbf{D}}{\partial t},
$$

From this relationship it is not difficult to switch over to the expression

$$
\text { โ] } \mathbf{H} d \mathbf{l}=\frac{\partial \Phi_{E}}{\partial t},
$$

where $\Phi_{E}=\int \mathbf{D} d \mathbf{s}$ the flow of electrical induction.

However, for the complete description of the processes of the mutual electrical induction of relationship (3.1) is insufficient. As in the case Faraday law, should be considered the circumstance that the flow of electrical induction can change not only due to the local derivative of electric field on the time, but also because the outline, along which is produced the integration, it can move in the threedimensional changing electric field. This means that in relationship (3.1), as in the case Faraday law, should be replaced the partial derivative by the complete. Designating by the primes of field and circuit elements in moving IMS, we will obtain:

$$
\text { † } \mathbf{H}^{\prime} d \mathbf{l}^{\prime}=\frac{d \Phi_{E}}{d t},
$$

and further

$$
\text { [f } \mathbf{H}^{\prime} d \mathbf{l}^{\prime}=\int \frac{\partial \mathbf{D}}{\partial t} d \mathbf{s}^{\prime}+\oint\left[[\mathbf{D} \times \mathbf{v}] d \mathbf{l}^{\prime}+\int \mathbf{v} \operatorname{div} \mathbf{D} d \mathbf{s}^{\prime} .\right.
$$

For the electrically neutral medium $\operatorname{div} \mathbf{E}=0$; therefore the last member of right side in this expression will be absent. For this case relationship (3.2) will take the form:

$$
\int \mathbf{H}^{\prime} d \mathbf{l}^{\prime}=\int \frac{\partial \mathbf{D}}{\partial t} d \mathbf{s}^{\prime}+\oint\left[[\mathbf{D} \times \mathbf{v}] d \mathbf{l}^{\prime} .\right.
$$

If we in this relationship pass from the contour integration to the integration for the surface, then we will obtain:

$$
\operatorname{rot} \mathbf{H}^{\prime}=\frac{\partial \mathbf{D}}{\partial t}+\operatorname{rot}[\mathbf{D} \times \mathbf{v}] .
$$

If we, based on this relationship, write down fields in this inertial system, then prime near $\mathbf{H}$ and second member of right side will disappear, and we will obtain the bias current, introduced by Maxwell. But Maxwell introduced this parameter, without resorting to the law of electromagnetic induction (3.2). If his law of magneto electric induction Faraday derived on the basis experiments with the magnetic fields, then experiments on the establishment of the validity of relationship (3.2) cannot be at that time conducted was, since. for conducting this experiment sensitivity of existing at that time meters did not be sufficient.

Fields on from (3.3) we obtain for the case of constant electrical:

$$
\mathbf{H}_{v}=-\varepsilon[\mathbf{v} \times \mathbf{E}] .
$$

Now electric fields can be expressed through the rotor of electrical vector potential, after assuming [8]

$$
\mathbf{E}=\operatorname{rot} \mathbf{A}_{E} .
$$

Relationship (3.4) taking into account (3.6) will be written down 


$$
\mathbf{H}^{\prime}=\varepsilon \frac{\partial \mathbf{A}_{E}}{\partial t}-\varepsilon\left[\mathbf{v} \times \operatorname{rot} \mathbf{A}_{E}\right] .
$$

Further it is possible to repeat all those procedures, which has already been conducted with the magnetic vector potential, and to write down the following relationships:

$$
\begin{gathered}
\mathbf{H}^{\prime}=\varepsilon \frac{\partial \mathbf{A}_{E}}{\partial t}+\varepsilon(\mathbf{v} \nabla) \mathbf{A}_{E}-\varepsilon \operatorname{grad}\left(\mathbf{v} \mathbf{A}_{E}\right), \\
\mathbf{H}^{\prime}=\varepsilon \frac{\partial \mathbf{A}_{E}}{\partial t}-\varepsilon\left[\mathbf{v} \times \operatorname{rot} \mathbf{A}_{E}\right], \\
\mathbf{H}^{\prime}=\varepsilon \frac{d A_{E}}{d t}-\varepsilon \operatorname{grad}\left(\mathbf{v} \mathbf{A}_{E}\right) .
\end{gathered}
$$

Is certain, the study of this problem it would be possible, as in the case the law of magneto electric induction, to begin from the introduction of the vector $\mathbf{A}_{E}$, but this way is specially passed traditionally, beginning from the integral law in order to show the identity of processes for two different laws, and the logical sequence of the introduction of the electrical vector of potentials.

The introduction of total derivatives in the laws of induction substantially explains physics of these processes and gives the possibility to isolate the force components, which act on the charge. This method gives also the possibility to obtain transformation laws fields on upon transfer of one IMS to another. Of this consists the modernization of old electrodynamics, although the physical essence of the introduced potentials remains, as earlier, it is not clear $[9,10]$.

\section{$\S 4$. Plurality of the forms of the writing of the electrodynamic laws}

In the previous paragraph it is shown that the magnetic and electric fields can be expressed through their vector potentials

$$
\begin{aligned}
& \mathbf{H}=\operatorname{rot} \mathbf{A}_{H}, \\
& \mathbf{E}=\operatorname{rot} \mathbf{A}_{E} .
\end{aligned}
$$

Consequently, Maxwell equations can be written down with the aid of these potentials:

$$
\begin{aligned}
& \operatorname{rot} \mathbf{A}_{E}=-\mu \frac{\partial \mathbf{A}_{H}}{\partial t}, \\
& \operatorname{rot} \mathbf{A}_{H}=\varepsilon \frac{\partial \mathbf{A}_{E}}{\partial t} .
\end{aligned}
$$

For each of these potentials it is possible to obtain wave equation, in particular

$$
\operatorname{rot} \operatorname{rot} \mathbf{A}_{E}=-\varepsilon \mu \frac{\partial^{2} \mathbf{A}_{E}}{\partial t^{2}}
$$

and to consider that in the space are extended not the magnetic and electric fields, but the field of electrical vector potential.

In this case, as can easily be seen of the relationships (4.1)-(4.4), magnetic and electric field they will be determined through this potential by the relationships: 


$$
\begin{aligned}
& \mathbf{H}=\varepsilon \frac{\partial \mathbf{A}_{E}}{\partial t} \\
& \mathbf{E}=\operatorname{rot} \mathbf{A}_{E}
\end{aligned}
$$

The gradient $\operatorname{rot} \mathbf{A}_{E}$ and local time derivative $\frac{\partial \mathbf{A}_{E}}{\partial t}$ are connected with wave equation (4.5).

Thus, the use only of one electrical vector potential makes it possible to completely solve the task about the propagation of electrical and magnetic fields on. Taking into account (4.6), Pointing vector can be written down only through the vector $\operatorname{rot} \mathbf{A}_{E}$ :

$$
\mathbf{P}=\varepsilon\left[\frac{\partial \mathbf{A}_{E}}{\partial t} \times \operatorname{rot} \mathbf{A}_{E}\right]
$$

Characteristic is the fact that with this method of examination necessary condition is the presence at the particular point of space both the time derivatives, and the gradients of one and the same potential.

This task can be solved by another method, after writing down wave equation for the magnetic vector potential:

$$
\operatorname{rot} \operatorname{rot} \mathbf{A}_{H}=-\varepsilon \mu \frac{\partial^{2} \mathbf{A}_{H}}{\partial t^{2}} .
$$

In this case magnetic and electric fields will be determined by the relationships

$$
\begin{aligned}
& \mathbf{H}=\operatorname{rot} \mathbf{A}_{H} \\
& \mathbf{E}=-\mu \frac{\partial \mathbf{A}_{H}}{\partial t} .
\end{aligned}
$$

The Pointing vector in this case can be found from the following relationship:

$$
\vec{P}=-\mu\left[\frac{\partial \mathbf{A}_{H}}{\partial t} \times \operatorname{rot} \mathbf{A}_{H}\right] \text {. }
$$

The gradient $\operatorname{rot} \mathbf{A}_{H}$ and local time derivative $\frac{\partial \mathbf{A}_{H}}{\partial t}$ are connected with wave equation (4.5).

But it is possible to enter and differently, after introducing, for example, the electrical and magnetic currents

$$
\begin{aligned}
& \mathbf{j}_{E}=\operatorname{rot} \mathbf{H}, \\
& \mathbf{j}_{H}=\operatorname{rot} \mathbf{E} .
\end{aligned}
$$

The equations also can be recorded for these currents:

$$
\begin{aligned}
& \operatorname{rot} \mathbf{j}_{H}=-\mu \frac{\partial \mathbf{j}_{E}}{\partial t}, \\
& \operatorname{rot} \mathbf{j}_{E}=\varepsilon \frac{\partial \mathbf{j}_{H}}{\partial t} .
\end{aligned}
$$

This system in its form and information concluded in it differs in no way from Maxwell equations, and it is possible to consider that in the space the magnetic or electric currents are extended. $[9,10]$. 


\section{CHAPTER 2}

\section{THE ROLE AND THE PLACE FOR THE KINETIC INDUCTANCE OF CHARGES IN THE CONTEMPORARY ELECTRODYNAMICS}

The classical electrodynamics of material media is one of the most important branches of physics not only on its theoretical, but also, in not smaller measure, to practical significance. Nevertheless, the traditional study even of this basic for it problem, as the frequency dispersion of electromagnetic waves [11-14], it does not manage without essential omissions and weak places.

\section{$\S 5$. Who and as introduced the frequency dispersion of the dielectric constant}

It is widely-known that physics is the quantitative science, based on the physical experiment, which is rested on the measurements, i.e., the comparison of the characteristics of the phenomena with the specific standards being investigated. For this in physics are introduced physical quantities, physical units of their measurement and meters. The experimentally obtained quantitative dependences make it possible to use mathematical methods for their working and to build the theoretical, i.e., mathematical models of the studied phenomena. Fundamental component of mathematical model are the functional dependences, which mutually connect different variables of the model accepted.

Such variables can be not only the physical quantities, but also the mathematical model parameters (briefly - the mathematical parameters), which play in the model auxiliary role. Mathematical models allow, among entire other, to quantitatively formulate (i.e., to formulate in the language of mathematics) physical laws, but in this case it is important that during the writing of physical law it is possible to use only physical quantities as the variables. This makes it possible to examine the physical sense of laws, since the mathematical parameters, in contrast to the physical quantities, are not allotted by physical sense. In particular, the mathematical parameter can be expressed creason they long time they were considered as the constants). It is considerably later, already at the beginning of this century with the explanation of the optical dispersion phenomena (in particular rainbow phenomenon). Heaviside and Wood showed that the dielectric and magnetic constants are the functions of frequency. But very recently, in the middle of the 50's, physics they came to the conclusion that these values depend not only on frequency, but also on the wave vector. On the essence, this was the existing ideas radical breaking. It was how a serious, is characterized the case, which occurred at the seminar L.D. Landau into 1954. During the report of A.I. Akhiezer on this theme Landau suddenly exclaimed, after smashing the speaker: "This is delirium, since the refractive index cannot be the function of refractive index". Note that this said L.D. Landau - one of the outstanding physicists of our time" (end of the quotation). 
It is incomprehensible from the given quotation, that precisely had in the form Landau. However, its subsequent publications speak, that it accepted this concept [12]. And again for some reason, following Heaviside and Wood, Landau did not introduce new name for the new mathematical parameter. Hardly this outstanding physicist XX of century could not understand this obvious thing, that the discussion deals precisely with the new mathematical parameter. It is faster, so it considered that misunderstandings it will not be.

However, a similar examination occurred in a number of fundamental works on electrodynamics [11-16], as a result what in physics solidly it was fastened this concept as the frequency dispersion of the dielectric constant of material media and, in particular, plasma. The propagation of this concept to the dielectrics led to the ideas about the fact that their dielectric constant also depends on frequency. There is the publications of such well-known scholars as the Drude, Wood, Heaviside, Landau, Ginsburg, Akhiezer, Tamm [11-16], where it is indicated that the dielectric constant of plasma and dielectrics depends on frequency.

Unfortunately, this caused many misunderstandings. Thus, many specialists cannot believe in the fact that the physical quantity of the relative dielectric constant of plasma is equal to the relative dielectric constant of vacuum, but the dispersion of the physical quantity of the dielectric constant of dielectrics is absent. However, main negative moment here lies in the fact that is not accentuated the attention of researchers in the urgency of the improvement of the mathematical models of the dispersion of electromagnetic waves in the direction of passage from the examination of the mathematical parameter by the name of dielectric constant to the examination of the physical quantity of relative dielectric constant.

The construction of such models of dispersion is possible only on the basis of a fundamental understanding of the physical sense of the proceeding processes. But precisely such models can describe those aspects of the phenomena, which previously proved to be inaccessible for the theoretical studies. Further we will show how the proper determination of the role and position for the kinetic inductance of charges in the electrodynamics it allows with the examination of the phenomenon of the dispersion of electromagnetic waves to limit to the use only of physical quantity of the relative dielectric constant of medium without the attraction of the corresponding mathematical parameters.

\section{§ 6. Plasma-like media}

By plasma media we will understand such, in which the charges can move without the losses. To such media in the first approximation, can be related the superconductors, free electrons or ions in the vacuum (subsequently conductors). In the absence magnetic field in the media indicated equation of motion for the electrons takes the form:

$$
m \frac{d \mathbf{v}}{d t}=e \mathbf{E},
$$

where $m$ - mass electron, $e$ - electron charge, $\mathbf{E}$ - tension of electric field, $\mathbf{v}$ speed of the motion of charge. 
In this equation is considered that the electron charge is negative. In [16] it is shown that this equation can be disseminated to the case of electron motion in the hot plasma.

Using an interrelation of the current densities and electrons

$$
\mathbf{j}=n e \mathbf{v} \text {, }
$$

from (6.1) we obtain the current density of the conductivity

$$
\mathbf{j}_{L}=\frac{n e^{2}}{m} \int \mathbf{E} d t
$$

After introducing the accordingly [ 8,9,17-19] specific kinetic inductance of charge carriers, whose existence is connected with the inertia properties of massive charge carriers,

$$
L_{k}=\frac{m}{n e^{2}},
$$

let us write down equality (6.3) in the form

$$
\mathbf{j}_{L}=\frac{1}{L_{k}} \int \mathbf{E} d t
$$

Fields on relationship (6.5) it will be written down for the case of harmonics:

$$
\mathbf{j}_{L}=-\frac{1}{\omega L_{k}} \mathbf{E}_{0} \cos \omega t \text {. }
$$

Here and throughout, as a rule, is used not the complex, but actual form of the record of electrodynamic formulas because of its clarity for the reflection of the phase relationships between the vectors, which represent electric fields and current densities.

From relationship (6.5) and (6.6) is evident that $\mathbf{j}_{L}$ presents inductive current, since. its phase is late with respect to the tension of electric field to the angle $\pi / 2$. If charges are located in the vacuum, then during the presence of summed current it is necessary to consider bias current

$$
\mathbf{j}_{\varepsilon}=\varepsilon_{0} \frac{\partial \mathbf{E}}{\partial t}=\omega \varepsilon_{0} \mathbf{E}_{0} \cos \omega t
$$

Is evident that this current bears capacitive nature, since. its phase anticipates the phase of the tension of electrical to the angle $\pi / 2$. Thus, summary current density will compose [17-22]

$$
\mathbf{j}_{\Sigma}=\varepsilon_{0} \frac{\partial \mathbf{E}}{\partial t}+\frac{1}{L_{k}} \int \mathbf{E} d t,
$$

or fields on for the case of harmonics

$$
\mathbf{j}_{\Sigma}=\left(\omega \varepsilon_{0}-\frac{1}{\omega L_{k}}\right) \mathbf{E}_{0} \cos \omega t .
$$

If electrons are located in the material medium, then in the general case should be still considered the presence of the positively charged ions, but rapidly changing in the particular case fields on their presence it is possible not to consider in connection with the significant exceeding of the mass of the ions above the mass of electrons. 
In (6.7) value in the brackets is summary susceptance of medium $\sigma_{\Sigma}$, that folding from the capacitive $\sigma_{C}$ and $\sigma_{L}$ inductive susceptance

$$
\sigma_{\Sigma}=\sigma_{C}+\sigma_{L}=\omega \varepsilon_{0}-\frac{1}{\omega L_{k}} .
$$

Relationship (6.7) can be rewritten and differently:

$$
\mathbf{j}_{\Sigma}=\omega \varepsilon_{0}\left(1-\frac{\omega_{0}^{2}}{\omega^{2}}\right) \mathbf{E}_{0} \cos \omega t,
$$

where $\omega_{0}=\sqrt{\frac{1}{L_{k} \varepsilon_{0}}}$ - plasma frequency of Langmuir vibrations.

The scalar quantity thus came out

$$
\varepsilon^{*}(\omega)=\varepsilon_{0}\left(1-\frac{\omega_{0}^{2}}{\omega^{2}}\right)=\varepsilon_{0}-\frac{1}{\omega^{2} L_{k}},
$$

which in the scientific literature, in particular, in the works on physics of plasma [11-16], is named the dielectric constant of plasma. If we treat this value, as the absolute dielectric constant of plasma in the sense that its relation to the electrical constant gives the physical quantity of the relative dielectric constant of plasma, then it will come out that the physical quantity of relative dielectric of the permeability of plasma depends on frequency. In the previous paragraph it was noted, that this is erroneous, and the obtained value is the certain mathematical parameter, which must be distinguished from the absolute and relative dielectric constant. In contrast to the absolute dielectric constant, which is conveniently called also in the more expanded version of designation physical absolute dielectric constant, the introduced value let us name effective absolute dielectric constant. It is analogous, in contrast to the relative dielectric constant, which is conveniently called also in the more expanded version of designation physical relative dielectric constant, let us name the ratio of the introduced value to the electrical constant effective relative dielectric constant. If the physical absolute and relative dielectric constants of medium do not depend on frequency, then the effective absolute and relative dielectric constants of medium on frequency depend.

It is important to note that the effective absolute dielectric constant of plasma proved to be the composite mathematical parameter, into which simultaneously enters electrical constant and specific kinetic inductance of the charges [22-24].

For further concrete definition of the examination of the dispersion of electromagnetic waves let us determine the concepts of the physical dielectric constants of medium (absolute and relative) for the case of variables fields on. Entering the second Maxwell equation summary current density (subsequently for the brevity we will use word "current" instead of "current density") in any medium is added only from following three components, which depend on the electric field:

1) the current of resistance losses there will be in-phase to electric field. 
2) the permittance current, called bias current (is determined by first-order derivative of electric field by the time and anticipates the tension of electric field on the phase on $\pi / 2$ ).

3 ) the conduction current, determined by integral of the electric field from the time, will lag behind the electric field on the phase to $\pi / 2$.

All these components must be present in any nonmagnetic regions with the heat losses. Therefore it is completely natural, the dielectric constant of any medium to define as the coefficient, confronting that term, which is determined by the derivative of electric field by the time in the second equation of Maxwell. In this case one should consider that this dielectric constant cannot be negative in connection with the fact that through it is determined energy of electrical fields on, but energy is always non-negative. Accordingly, physical relative dielectric constant is equal to the ratio of physical absolute dielectric constant to the electrical constant. Let us generally note that both for the effective and for the physical dielectric constant acts the trivial general rule - the relative permeability is always equal to the ratio of absolute permeability to the electrical constant, so that word "absolute" or "relative" we will for the brevity as far as possible omit.

The proposed mathematical model of the dispersion of electromagnetic waves in the plasma is differed from the previously known the fact that not the effective, but physical dielectric constant of plasma is used. This becomes possible due to the calculation of the kinetic inductance of charges on the basis of the deep understanding of the physical sense of dispersion. As a result, the proposed model makes it possible to consider initial conditions during the solution of integrodifferential equation for the current by means of the introduction to the appropriate integration constant.

However, the physical dielectric constant of plasma in the ac fields is not determined with the traditional examination and even current is not spread to the bias current and the conduction current, one of which is determined electrical constant and derivative of electric field, but another is determined by specific kinetic inductance and integral of the electric field. To a certain degree this "dumping of currents into the total heap" is justified, since derivative and integral of the function of harmonic oscillation are distinguished only by sign. Let us emphasize that from a mathematical point of view to reach in the manner that it entered to Landau, it is possible, but in this case is lost the integration constant, which is necessary to account for initial conditions during the solution of the equation, which determines current density in the material medium.

The separation of currents in the proposed model makes it possible to better understand physics of phenomenon. One of these two antiphase competing currents depends on frequency linearly, another - it is inversely proportional to frequency. The conduction current predominates with the low frequencies, the bias current, on the contrary, predominates with the high. At the plasma current frequency are equal and enter into the resonance with each other.

Analogous with introduction to effective dielectric constant it is possible to introduce the effective (different from the physical) kinetic inductance depending on the frequency 


$$
L_{k}^{*}(\omega)=\frac{L_{k}}{\left(1-\frac{\omega^{2}}{\omega_{0}^{2}}\right)}=\frac{L_{k}}{1-\varepsilon_{0} \omega^{2} L_{k}},
$$

after writing down relationship (6.7) in the form:

$$
\mathbf{j}_{\Sigma}=-\frac{\left(1-\frac{\omega^{2}}{\omega_{0}^{2}}\right)}{\omega L_{k}} \mathbf{E}_{0} \cos \omega t .
$$

The parameters $\varepsilon^{*}(\omega), L_{k}^{*}(\omega)$ make it possible to write down (6.7) in two equivalent forms:

$$
\begin{aligned}
& \mathbf{j}_{\Sigma}=\omega \varepsilon^{*}(\omega) \mathbf{E}_{0} \cos \omega t, \\
& \mathbf{j}_{\Sigma}=-\frac{1}{\omega L_{k}^{*}(\omega)} \mathbf{E}_{0} \cos \omega t .
\end{aligned}
$$

The first of these parameters is equal to the ratio of summary susceptance of medium to the frequency, and the second is equal with the sign "minus" to the reciprocal value of the work of frequency and of susceptance of the medium:

$$
\varepsilon^{*}(\omega)=\frac{\sigma_{\Sigma}}{\omega}, L_{k}^{*}(\omega)=-\frac{1}{\omega \sigma_{\Sigma}} .
$$

Natural to substitute these values in the formulas, which determine energy of electrical fields on

$$
W_{E}=\frac{1}{2} \varepsilon_{0} E_{0}^{2},
$$

and kinetic energy of charge carriers

$$
W_{j}=\frac{1}{2} L_{k} j_{0}^{2}
$$

it is simple because in these formulas not the effective, but corresponding physical quantities figure. It is not difficult to show that in this case the total specific energy can be obtained from the relationship

$$
W_{\Sigma}=\frac{1}{2} \cdot \frac{d\left(\omega \varepsilon^{*}(\omega)\right)}{d \omega} E_{0}^{2},
$$

from which we obtain

$$
W_{\Sigma}=\frac{1}{2} \varepsilon_{0} E_{0}^{2}+\frac{1}{2} \frac{1}{\omega^{2} L_{k}} E_{0}^{2}=\frac{1}{2} \varepsilon_{0} E_{0}^{2}+\frac{1}{2} L_{k} j_{0}^{2} .
$$

We will obtain the same result, after using the formula

$$
W=\frac{1}{2} \frac{d\left[\frac{1}{\omega L_{k}^{*}(\omega)}\right]}{d \omega} E_{0}^{2} .
$$

The given relationships show that the specific energy consists of potential energy of electrical fields on and to kinetic energy of charge carriers.

Wave equation follows from the appropriate system of Maxwell equations, which completely describes the electrodynamics of the no dissipative conductors: 


$$
\begin{aligned}
& \operatorname{rot} \mathbf{E}=-\mu_{0} \frac{\partial \mathbf{H}}{\partial t}, \\
& \operatorname{rot} \mathbf{H}=\varepsilon_{0} \frac{\partial \mathbf{E}}{\partial t}+\frac{1}{L_{k}} \int \mathbf{E} d t
\end{aligned}
$$

where $\varepsilon_{0}, \mu_{0}$ - electrical and magnetic constants.

From (6.10) we obtain

$$
\operatorname{rot} \operatorname{rot} \mathbf{H}+\mu_{0} \varepsilon_{0} \frac{\partial^{2} \mathbf{H}}{\partial t^{2}}+\frac{\mu_{0}}{L_{k}} \mathbf{H}=0 .
$$

For the case fields on, time-independent, equation (6.11) passes into the equation of London

$$
\operatorname{rot} \operatorname{rot} \mathbf{H}+\frac{\mu_{0}}{L_{k}} \mathbf{H}=0,
$$

where $\lambda_{L}^{2}=\frac{L_{k}}{\mu_{0}}$ - London depth of penetration.

Thus, it is possible to conclude that the London equations [25] being a special case of equation (6.11), and do not consider bias currents on medium. Therefore they do not give the possibility to obtain the wave equations, which describe the processes of the propagation of electromagnetic waves in the superconductors.

For the electrical fields on the wave equation of signs the form:

$$
\operatorname{rot} \operatorname{rot} \mathbf{E}+\mu_{0} \varepsilon_{0} \frac{\partial^{2} \mathbf{E}}{\partial t^{2}}+\frac{\mu_{0}}{L_{k}} \mathbf{E}=0 .
$$

For the variable electrical fields we have

$$
\operatorname{rot} \operatorname{rot} \mathbf{E}+\frac{\mu_{0}}{L_{k}} \mathbf{E}=0 .
$$

Consequently, dc fields penetrate the superconductor in the same manner as for magnetic, diminishing exponentially. However, the density of current in this case grows according to the linear law

$$
\mathbf{j}_{L}=\frac{1}{L_{k}} \int \mathbf{E} d t .
$$

It is evident from the developed mathematical model of dispersion that the physical absolute dielectric constant of this medium is connected with the accumulation of potential energy, it does not depend on frequency and it is equal to the physical absolute dielectric constant of vacuum, i.e., by electrical constant. Furthermore, this medium is characterized still and the kinetic inductance of charge carriers and this parameter determines the kinetic energy, accumulated on medium.

Thus, in contrast to the conventional procedure [12-13] of the examination of the process of the propagation of electromagnetic waves in no dissipative conducting media, the proposed procedure does not require the introduction of polarization vector, but equation of motion is assumed as the basis of examination in it, and in this case in the second equation of Maxwell are extracted all components of current densities explicitly. 
For further understanding of physical nature of the phenomenon of dispersion we will use the simple radio-technical method of equivalent diagrams, which makes it possible to clearly present in the form such diagrams not only radio-technical elements with the concentrated and distributed parameters, but also material media. As it will be shown below, according to this method, the single volume of conductor or plasma according to its electrodynamic characteristics is equivalent to parallel resonant circuit with the lumped parameters. Let us examine parallel resonant circuit with the parallel connection of capacity $C$ and inductance $L$. The connection between the voltage $U$, applied to the outline, and the summed current $I_{\Sigma}$, which flows through this chain, takes the form

$$
I_{\Sigma}=I_{C}+I_{L}=C \frac{d U}{d t}+\frac{1}{L} \int U d t,
$$

where $I_{C}=C \frac{d U}{d t}, I_{L}=\frac{1}{L} \int U d t-$ the currents, which flow through the capacity and the inductance respectively.

We obtain for the alternating voltage according to the harmonic $U=U_{0} \sin \omega t$ law

$$
I_{\Sigma}=\left(\omega C-\frac{1}{\omega L}\right) U_{0} \cos \omega t .
$$

In (6.12) value in the brackets there is summary susceptance $\sigma_{\Sigma}$ of chain, which consists of the capacitive $\sigma_{C}$ and $\sigma_{L}$ inductive susceptance

$$
\sigma_{\Sigma}=\sigma_{C}+\sigma_{L}=\omega C-\frac{1}{\omega L} \text {. }
$$

In this case relationship (6.12) can be rewritten as follows:

$$
I_{\Sigma}=\omega C\left(1-\frac{\omega_{0}^{2}}{\omega^{2}}\right) U_{0} \cos \omega t,
$$

where $\omega_{0}^{2}=\frac{1}{L C}$ - the resonance frequency of parallel circuit.

As in the case conductors, it is possible to introduce the new mathematical parameter of the effective capacity

$$
C^{*}(\omega)=C\left(1-\frac{\omega_{0}^{2}}{\omega^{2}}\right)=C-\frac{1}{\omega^{2} L}
$$

depending on the frequency, capacity and even inductance and susceptance of chain to the frequency equal to relation. And it is again necessary this mathematical parameter to distinguish from the physical capacity, which is conventionally designated as simply the capacity, and which is not the mathematical parameter, but physical quantity.

Relationship (6.12) can be rewritten and differently:

$$
I_{\Sigma}=-\frac{\left(1-\frac{\omega^{2}}{\omega_{0}^{2}}\right)}{\omega L} U_{0} \cos \omega t,
$$

after introducing the new mathematical parameter of the effective inductance 


$$
L^{*}(\omega)=\frac{L}{\left(1-\frac{\omega^{2}}{\omega_{0}^{2}}\right)}=\frac{L}{1-\omega^{2} L C} .
$$

It is the reciprocal value of the work of summary susceptance and frequency with the sign "minus".

Using expressions (6.13) and (6.14), let us write down:

$$
\begin{gathered}
I_{\Sigma}=\omega C^{*}(\omega) U_{0} \cos \omega t, \\
I_{\Sigma}=-\left(1 /\left(\omega L^{*}(\omega)\right)\right) U_{0} \cos \omega t .
\end{gathered}
$$

Relationships (6.15) and (6.16), using the different parameters $C^{*}(\omega)$ and $L^{*}(\omega)$, they are equivalent, and each of them completely characterizes chain.

Accumulated in the capacity and the inductance energy, is determined from the relationships

$$
\begin{aligned}
W_{C} & =\frac{1}{2} C U_{0}^{2}, \\
W_{L} & =\frac{1}{2} L I_{0}{ }^{2} .
\end{aligned}
$$

It is interesting that if we into the formulas (6.17), (6.18) instead of the physical of capacity and inductance substitute the appropriate effective values (6.13), (6.14), that it will come out that energy can be negative. The so-called problem of negative energy, which is inherent in a whole series of the mathematical models of frequency dispersion, including to Klein-Gordon equations for the scalar massive particles and Dirac for the fermions in quantum physics, appears. However, in the case of parallel resonant circuit it is obvious that the problem indicated is obliged to its appearance to the incorrect replacement of physical quantities to the appropriate effective mathematical parameters. This gives the specific orientators for the more in-depth research of the problem of negative energy in the different models of the frequency dispersion, including of quantum, but these questions already they exceed the scope of the thematic of this monograph.

It is easy to see that the summary energy, accumulated in the outline, can be expressed by the mutually equivalent equalities:

$$
\begin{aligned}
& W_{\Sigma}=\frac{1}{2} \frac{d \sigma_{X}}{d \omega} U_{0}^{2}, \\
& W_{\Sigma}=\frac{1}{2} \frac{d\left[\omega C^{*}(\omega)\right]}{d \omega} U_{0}^{2}, \\
& W_{\Sigma}=\frac{1}{2} \frac{d\left(\frac{1}{\omega L^{*}(\omega)}\right)}{d \omega} U_{0}{ }^{2} .
\end{aligned}
$$

Any of the equalities (6.19)-(6.21) gives the identical result:

$$
W_{\Sigma}=C U_{0}^{2} / 2+L I_{0}^{2} / 2
$$

where $U_{0}$ - amplitude of voltage on the capacity, and $I_{0}$ - amplitude of the current, which flows through the inductance.

Thus, parallel resonant circuit can be mathematically simulated from three mutually equivalent points of view: 
1) physical capacity and physical inductance form outline.

2) outline is described by the frequency-dependent effective capacity.

3) outline is described by the frequency-dependent effective inductance.

In the quasi-static regime electrodynamic processes in the conductors are similar to processes in the parallel resonant circuit with the lumped parameters. Relationships for the parallel resonant circuit are identical to relationships for the conductors during the replacement: $\quad E_{0} \rightarrow U_{0}, j_{0} \rightarrow I_{0}, \varepsilon_{0} \rightarrow C, L_{k} \rightarrow L$. Thus, the single volume of conductor, with the uniform distribution of electrical fields on and current densities in it, it is equivalent to parallel resonant circuit with the lumped parameters indicated. In this case the capacity of this outline is numerically equal to the dielectric constant of vacuum, and inductance is equal to the specific kinetic inductance of charges.

This approach does not require introduction into the examination of polarization vector in the conductors in contrast to the conventional procedure [12-15]. In particular, the paragraph 59 of work [12] begins with the words: "We pass now to the study of the most important question about the rapidly changing electric fields, whose frequencies are unconfined by the condition of smallness in comparison with the frequencies, characteristic for establishing the electrical and magnetic polarization of substance" (end of the quotation). These words mean that that region of the frequencies, where, in connection with the presence of the inertia properties of charge carriers, the polarization of substance will not reach its static values, is examined. With the further consideration of a question is done the conclusion that "in any variable field, including with the presence of dispersion, the polarization vector $\mathbf{P}=\mathbf{D}-\varepsilon_{0} \mathbf{E}$ (here and throughout all formulas cited they are written in the system SI) preserves its physical sense of the electric moment of the unit volume of substance" (end of the quotation). Let us give the still one quotation [12]: "It proves to be possible to establish (unimportantly - metals or dielectrics) maximum form of the function $\varepsilon(\omega)$ with the high frequencies valid for any bodies. Specifically, the field frequency must be great in comparison with "the frequencies" of the motion of all (or, at least, majority) electrons in the atoms of this substance. With the observance of this condition it is possible with the calculation of the polarization of substance to consider electrons as free, disregarding their interaction with each other and with the atomic nuclei" (end of the quotation).

Further, as this is done and in this work, is written the equation of motion of free electron in the ac field [12]:

$$
m \frac{d \mathbf{v}}{d t}=e \mathbf{E},
$$

from where its displacement is located

$$
\mathbf{r}=-\frac{e \mathbf{E}}{m \omega^{2}} .
$$

Then is indicated that the polarization $\mathbf{P}$ is a dipole moment of unit volume and the obtained displacement is put into the polarization.

$$
\mathbf{P}=n e \mathbf{r}=-\frac{n e^{2} \mathbf{E}}{m \omega^{2}} .
$$


In this case point charge is examined, and this operation indicates the introduction of electrical dipole moment for two point charges with the opposite signs, located at a distance $\mathbf{r}$

$$
\mathbf{p}_{e}=-e \mathbf{r},
$$

where the vector $\mathbf{r}$ is directed from the negative charge toward the positive charge. This step causes bewilderment, since the point electron is examined, and in order to speak about the electrical dipole moment, it is necessary to have in this medium for each electron another charge of opposite sign, referred from it to the distance of In this case is examined the gas of free electrons, in which there are no charges of opposite signs. Further follows the standard procedure, when introduced thus illegal polarization vector is introduced into the dielectric constant

$$
\mathbf{D}=\varepsilon_{0} \mathbf{E}+\mathbf{P}=\varepsilon_{0} \vec{E}-\frac{n e^{2} \mathbf{E}}{m \omega^{2}}=\varepsilon_{0}\left(1-\frac{1}{\varepsilon_{0} L_{k} \omega^{2}}\right) \mathbf{E} .
$$

And since plasma frequency is determined by the relationship

$$
\omega_{p}^{2}=\frac{1}{\varepsilon_{0} L_{k}} .
$$

The vector of the induction immediately is written

$$
\mathbf{D}=\varepsilon_{0}\left(1-\frac{\omega_{p}^{2}}{\omega^{2}}\right) \mathbf{E} .
$$

With this approach it turns out that constant of proportionality

$$
\varepsilon(\omega)=\varepsilon_{0}\left(1-\frac{\omega_{p}^{2}}{\omega^{2}}\right),
$$

between the electric field and the electrical induction, named dielectric constant, depends on frequency, and following it and electrical induction was declared depending on the frequency [12-16]. But, as it was shown above, this mathematical parameter is not physical absolute dielectric constant, but ratio of summary susceptance of medium to the frequency.

Further into $\S 61$ of work [12] is examined a question about the energy of electrical and magnetic field in the media, which possess by the so-called dispersion. In this case is done the conclusion that relationship for the energy of such fields

$$
W=\frac{1}{2}\left(\varepsilon E_{0}^{2}+\mu H_{0}^{2}\right) \text {, }
$$

that making precise thermodynamic sense in the usual media, with the presence of dispersion so interpreted be cannot. These words mean that the knowledge of real electrical and magnetic fields on medium with the dispersion insufficiently for determining the difference in the internal energy per unit of volume of substance in the presence fields on in their absence. After this assertion is given the formula, which gives the same result for enumerating the specific energy of electrical and magnetic fields on with the presence of dispersion, that also the proposed in this monograph approac 


$$
W=\frac{1}{2} \frac{d(\omega \varepsilon(\omega))}{d \omega} E_{0}^{2}+\frac{1}{2} \frac{d(\omega \mu(\omega))}{d \omega} H_{0}^{2}
$$

First term in the right side (6.23) corresponds (6.9), and it means it is the total energy, which includes not only potential energy of electrical fields on, but also kinetic energy of the moving charges. This confirms conclusion about the impossibility of the interpretation precisely of formula (6.22), as the internal energy of electrical and magnetic fields on in the dispersive media, although this interpretation in the media in principle examined is possible. It consists in the fact that for the definition of the value of specific energy as the thermodynamic parameter in this case is necessary to correctly calculate this energy, taking into account not only electric field, which accumulates potential energy, but also current of the conduction electrons, which accumulate the kinetic kinetic energy of charges (6.8).

\section{$\S 7$. Transverse plasma resonance}

Development of mathematical models of dispersion of electromagnetic waves in conducting media, use physical permittivity of the plasma, allows to put theoretically substantiated the hypothesis of the existence of a new physical phenomenon. It can be called a cross-plasma resonance in non-magnetized plasma. This phenomenon is not only of great theoretical interest, but also may have important technical applications [26, 27].

Is known that the plasma resonance is longitudinal. But longitudinal resonance cannot emit transverse electromagnetic waves. However, with the explosions of nuclear charges, as a result of which is formed very hot plasma, occurs electromagnetic radiation in the very wide frequency band, up to the long-wave radiofrequency band. Today are not known those of the physical mechanisms, which could explain the appearance of this emission. On existence in the no magnetized plasma of any other resonances, except Langmuir, earlier known it was not, but it occurs that in the confined plasma the transverse resonance can exist, and the frequency of this resonance coincides with the frequency of Langmuir resonance, i.e. these resonances are degenerate. Specifically, this resonance can be the reason for the emission of electromagnetic waves with the explosions of nuclear charges. For explaining the conditions for the excitation of this resonance let us examine the long line, which consists of two ideally conducting planes, as shown in Fig.2.

Linear capacity and inductance of this line without taking into account edge effects they are determined by the relationships:

$$
C_{0}=\varepsilon_{0} \frac{b}{a}, L_{0}=\mu_{0} \frac{a}{b}
$$

Therefore with an increase in the length of line its total capacitance $C_{\Sigma}=\varepsilon_{0} \frac{b}{a} z$ and summary inductance $L_{\Sigma}=\mu_{0} \frac{a}{b} z$ increase proportional to its length. 


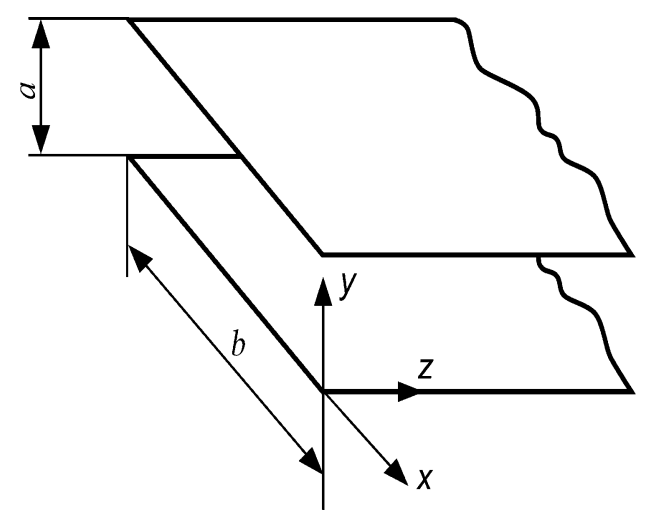

Fig. 2. The two-wire circuit, which consists of two ideally conducting planes

If we into the extended line place the plasma, charge carriers in which can move without the losses, and in the transverse direction pass through the plasma the current $I$, then charges, moving with the definite speed, will accumulate kinetic energy. Let us note that here are not examined technical questions, as and it is possible confined plasma between the planes of line how. In this case only fundamental questions, which are concerned transverse plasma resonance in the nonmagnetic plasma, are examined.

Since the transverse current density in this line is determined by the relationship

$$
j=\frac{I}{b z}=n e v .
$$

The summary kinetic energy of the moving charges can be written down

$$
W_{k \Sigma}=\frac{1}{2} \frac{m}{n e^{2}} a b z j^{2}=\frac{1}{2} \frac{m}{n e^{2}} \frac{a}{b z} I^{2} .
$$

Relationship (7.1) connects the kinetic energy, accumulated in the line, with the square of current; therefore the coefficient, which stands in the right side of this relationship before the square of current, is the summary kinetic inductance of line.

$$
L_{k \Sigma}=\frac{m}{n e^{2}} \cdot \frac{a}{b z} \text {. }
$$

Thus, the value

$$
L_{k}=\frac{m}{n e^{2}}
$$

presents the specific kinetic inductance of charges. This value was already previously introduced by another method (see relationship (6.4)). Relationship (7.3) is obtained for the case of the direct current, when current distribution is uniform.

Subsequently for the larger clarity of the obtained results, together with their mathematical idea, we will use the method of equivalent diagrams. The section, the lines examined, long $d z$ can be represented in the form the equivalent diagram, shown in Fig. 3 (a).

From relationship (7.2) is evident that in contrast to $C_{\Sigma}, L_{\Sigma}$ the value $L_{k \Sigma}$ with an increase in $z$ does not increase, but it decreases. Connected this with the fact that with an increase in $z$ a quantity of parallel-connected inductive elements grows. 


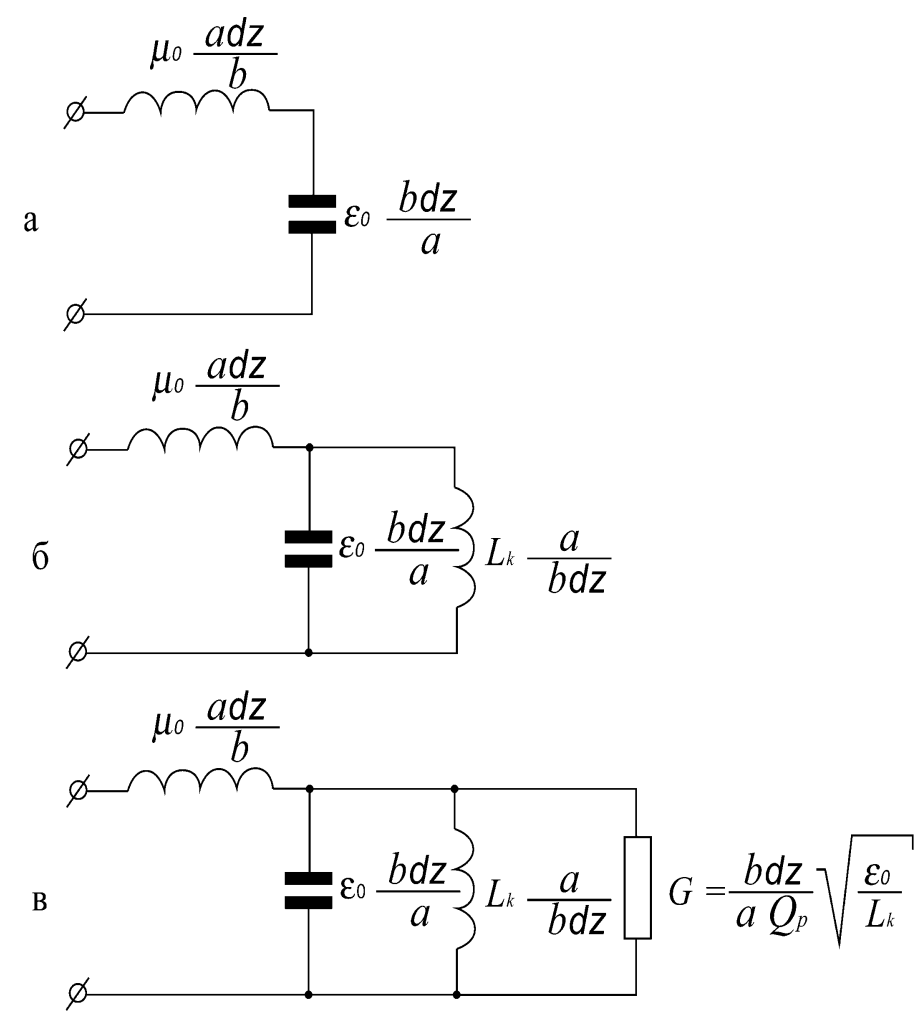

Fig. 3. a - the equivalent the schematic of the section of the two-wire circuit,

$\sigma$ - the equivalent the schematic of the section of the two-wire circuit, filled with no dissipative plasma,

в - the equivalent the schematic of the section of the two-wire circuit, filled with dissipative plasma

The equivalent the schematic of the section of the line, filled with no dissipative plasma, it is shown in Fig. 3 (б). Line itself in this case will be equivalent to parallel circuit with the lumped parameters:

$$
\begin{aligned}
& C=\frac{\varepsilon_{0} b z}{a}, \\
& L=\frac{L_{k} a}{b z}
\end{aligned}
$$

in series with which is connected the inductance

$$
\mu_{0} \frac{a d z}{b} \text {. }
$$

But if we calculate the resonance frequency of this outline, then it will seem that this frequency generally not on what sizes depends, actually:

$$
\omega_{\rho}^{2}=\frac{1}{C L}=\frac{1}{\varepsilon_{0} L_{k}}=\frac{n e^{2}}{\varepsilon_{0} m} .
$$

Is obtained the very interesting result, which speaks, that the resonance frequency macroscopic of the resonator examined does not depend on its sizes. Impression can be created, that this is plasma resonance, since. the obtained value of resonance frequency exactly corresponds to the value of this resonance. But it is known that 
the plasma resonance characterizes longitudinal waves in the long line they, while occur transverse waves. In the case examined the value of the phase speed in the direction of $z$ is equal to infinity and the wave vector $\vec{k}=0$.

This result corresponds to the solution of system of equations (6.10) for the line with the assigned configuration. In this case the wave number is determined by the relationship:

$$
k_{z}^{2}=\frac{\omega^{2}}{c^{2}}\left(1-\frac{\omega_{\rho}^{2}}{\omega^{2}}\right)
$$

and the group and phase speeds

$$
\begin{aligned}
& v_{g}^{2}=c^{2}\left(1-\frac{\omega_{\rho}^{2}}{\omega^{2}}\right), \\
& v_{F}^{2}=\frac{c^{2}}{\left(1-\frac{\omega_{\rho}^{2}}{\omega^{2}}\right)},
\end{aligned}
$$

where $c=\left(\frac{1}{\mu_{0} \varepsilon_{0}}\right)^{1 / 2}$ - speed of light in the vacuum.

For the present instance the phase speed of electromagnetic wave is equal to infinity, which corresponds to transverse resonance at the plasma frequency. Consequently, at each moment of time fields on distribution and currents in this line uniform and it does not depend on the coordinate $z$, but current in the planes of line in the direction of is absent. This, from one side, it means that the inductance $L_{\Sigma}$ will not have effects on electrodynamic processes in this line, but instead of the conducting planes can be used any planes or devices, which limit plasma on top and from below.

From relationships (7.4), (7.5) and (7.6) is evident that at the point $\omega=\omega_{p}$ occurs the transverse resonance with the infinite quality. With the presence of losses in the resonator will occur the damping, and in the long line in this case $k_{z} \neq 0$, and in the line will be extended the damped transverse wave, the direction of propagation of which will be normal to the direction of the motion of charges. It should be noted that the fact of existence of this resonance is not described by other authors.

Before to pass to the more detailed study of this problem, let us pause at the energy processes, which occur in the line in the case of the absence of losses examined.

Fields on the characteristic impedance of plasma, which gives the relation of the transverse components of electrical and magnetic, let us determine from the relationship

$$
Z=\frac{E_{y}}{H_{x}}=\frac{\mu_{0} \omega}{k_{z}}=Z_{0}\left(1-\frac{\omega_{\rho}^{2}}{\omega^{2}}\right)^{-1 / 2},
$$


where $Z_{0}=\sqrt{\frac{\mu_{0}}{\varepsilon_{0}}}$ - characteristic impedance of vacuum.

The obtained value $Z$ is characteristic for the transverse electrical waves in the waveguides. It is evident that when $\omega \rightarrow \omega_{p}$, then $Z \rightarrow \infty$, and $H_{x} \rightarrow 0$. When $\omega$ $>\omega_{p}$ in the plasma there is electrical and magnetic component of field. The energy densityof these fields on it will be written down:

$$
W_{E, H}=\frac{1}{2} \varepsilon_{0} E_{0 y}^{2}+\frac{1}{2} \mu_{0} H_{0 x}^{2} \text {. }
$$

Thus, the energy density, concluded in the magnetic field, in $\left(1-\frac{\omega_{\rho}^{2}}{\omega^{2}}\right)$ of times is less than the energy density, concluded in the electric field. Let us note that this examination, which is traditional in the electrodynamics, is not complete, since. in this case is not taken into account one additional form of energy, namely kinetic energy of charge carriers. Occurs that fields on besides the waves of electrical and magnetic, that carry electrical and magnetic energy, in the plasma there exists even and the third - kinetic wave, which carries kinetic energy of current carriers. The specific energy of this wave is written:

$$
W_{k}=\frac{1}{2} L_{k} j_{0}^{2}=\frac{1}{2} \cdot \frac{1}{\omega^{2} L_{k}} E_{0}^{2}=\frac{1}{2} \varepsilon_{0} \frac{\omega_{\rho}^{2}}{\omega^{2}} E_{0}^{2} .
$$

Consequently, the total energy density of wave is written as

$$
W_{E, H, j}=\frac{1}{2} \varepsilon_{0} E_{0 y}^{2}+\frac{1}{2} \mu_{0} H_{0 x}^{2}+\frac{1}{2} L_{k} j_{0}^{2} .
$$

Thus, for finding the total energy density, by the prisoner per unit of volume of plasma, calculation only fields on $E$ and $H$ it is insufficient.

At the point $\omega=\omega_{p}$ are carried out the relationship:

$$
\begin{aligned}
& W_{H}=0 \\
& W_{E}=W_{k}
\end{aligned},
$$

and magnetic field in the plasma is absent, and plasma presents macroscopic electromechanical resonator with the infinite quality, resounding at the frequency $\omega_{p}$.

Since with the frequencies $\omega>\omega_{p}$ the wave, which is extended in the plasma, it bears on itself three forms of the energy: electrical, magnetic and kinetic, then this wave can be named electricmagnetickinetic wave/ Kinetic wave is the wave of the current density $\mathbf{j}=\frac{1}{L_{k}} \int \mathbf{E} d t$. This wave is moved with respect to the electrical wave the angle $\pi / 2$.

Until now considered unrealizable case where the loss of plasma available, which corresponds to the infinite resonator Q-factor plasma.

Until now considered unrealizable case where the loss of plasma available, which corresponds to the infinite quality factor of plasma resonator. If losses are located, moreover completely it does not have value, by what physical processes such loss- 
es are caused, then the quality of plasma resonator will be finite quantity. For this case Maxwell equations they will take the form:

$$
\begin{aligned}
& \operatorname{rot} \mathbf{E}=-\mu_{0} \frac{\partial \mathbf{H}}{\partial t}, \\
& \operatorname{rot} \vec{H}=\sigma_{p . e f} \mathbf{E}+\varepsilon_{0} \frac{\partial \mathbf{E}}{\partial t}+\frac{1}{L_{k}} \int \mathbf{E} d t .
\end{aligned}
$$

The presence of losses is considered by the term $\mathbf{E}$, and, using near the conductivity of the index ef, it is thus emphasized that us does not interest very mechanism of losses, but only very fact of their existence interests. The value $\sigma_{e f}$ determines the quality factor of plasma resonator. For measuring $\sigma_{e f}$ should be selected the section of line by the length $z_{0}$, whose value is considerably lower than the wavelength in the plasma. This section will be equivalent to outline with the lumped parameters:

$$
\begin{aligned}
C & =\varepsilon_{0} \frac{b z_{0}}{a}, \\
L & =L_{k} \frac{a}{b z_{0}}, \\
G & =\sigma_{\rho . e f} \frac{b z_{0}}{a},
\end{aligned}
$$

where $G$ - conductivity, connected in parallel $C$ and $L$.

Conductivity and Q-factor in this outline enter into the relationship:

$$
G=\frac{1}{Q_{\rho}} \sqrt{\frac{C}{L}},
$$

from where, taking into account (7.8)-(7.10), we obtain

$$
\sigma_{\rho . e f}=\frac{1}{Q_{\rho}} \sqrt{\frac{\varepsilon_{0}}{L_{k}}} .
$$

Thus, measuring its own quality plasma of the resonator examined, it is possible to determine $\sigma_{p . e f}$. Using (7.2) and (7.11) we obtain:

$$
\begin{aligned}
& \operatorname{rot} \mathbf{E}=-\mu_{0} \frac{\partial \mathbf{H}}{\partial t}, \\
& \operatorname{rot} \mathbf{H}=\frac{1}{Q_{\rho}} \sqrt{\frac{\varepsilon_{0}}{L_{k}}} \mathbf{E}+\varepsilon_{0} \frac{\partial \mathbf{E}}{\partial t}+\frac{1}{L_{k}} \int \mathbf{E} d t .
\end{aligned}
$$

The equivalent the schematic of this line, filled with dissipative plasma, is represented in Fig. 3 (в).

Let us examine the solution of system of equations (7.12) at the point $\omega=\omega_{p}$, in this case, since

$$
\varepsilon_{0} \frac{\partial \mathbf{E}}{\partial t}+\frac{1}{L_{k}} \int \mathbf{E} d t=0
$$

we obtain 


$$
\begin{aligned}
& \operatorname{rot} \mathbf{E}=-\mu_{0} \frac{\partial \mathbf{H}}{\partial t}, \\
& \operatorname{rot} \mathbf{H}=\frac{1}{Q_{P}} \sqrt{\frac{\varepsilon_{0}}{L_{k}}} \mathbf{E} .
\end{aligned}
$$

These relationships determine wave processes at the point of resonance.

If losses in the plasma, which fills line are small, and strange current source is connected to the line, then it is possible to assume:

$$
\begin{aligned}
& \operatorname{rot} \mathbf{E} \cong 0, \\
& \frac{1}{Q_{p}} \sqrt{\frac{\varepsilon_{0}}{L_{k}}} \mathbf{E}+\varepsilon_{0} \frac{\partial \mathbf{E}}{\partial t}+\frac{1}{L_{k}} \int \mathbf{E} d t=\mathbf{j}_{C T},
\end{aligned}
$$

where $\mathbf{j}_{C T}$ - density of strange currents.

After integrating (7.13) with respect to the time and after dividing both parts to $\varepsilon_{0}$, we will obtain

$$
\omega_{p}^{2} \mathbf{E}+\frac{\omega_{p}}{Q_{p}} \cdot \frac{\partial \mathbf{E}}{\partial t}+\frac{\partial^{2} \mathbf{E}}{\partial t^{2}}=\frac{1}{\varepsilon_{0}} \cdot \frac{\partial \mathbf{j}_{C T}}{\partial t} .
$$

If we relationship (7.14) integrates over the surface of normal to the vector $\mathbf{E}$ and to introduce the electric flux $\Phi_{E}=\int \mathbf{E} d \mathbf{s}$ we will obtain:

$$
\omega_{p}^{2} \Phi_{E}+\frac{\omega_{p}}{Q_{p}} \cdot \frac{\partial \Phi_{E}}{\partial t}+\frac{\partial^{2} \Phi_{E}}{\partial t^{2}}=\frac{1}{\varepsilon_{0}} \cdot \frac{\partial I_{C T}}{\partial t}
$$

where $I_{C T}$ - strange current.

The equation (7.15) is the equation of harmonic oscillator with the right side, characteristic for the two-level laser [28]. If the source of excitation was opened, then relationship (7.14) presents "cold" laser resonator, in which the fluctuations will attenuate exponentially

$$
\Phi_{E}(t)=\Phi_{E}(0) e^{i \omega_{P} t} \cdot e^{-\frac{\omega_{P}}{2 Q_{P}} t},
$$

and the macroscopic electric flux $\Phi_{E}(t)$ will oscillate with the frequency $\omega_{p}$, relaxation time in this case is determined by the relationship:

$$
\tau=\frac{2 Q_{P}}{\omega_{P}} .
$$

The problem of developing of laser consists to now only in the skill excite this resonator.

If resonator is excited by strange currents, then this resonator presents band-pass filter with the resonance frequency to equal plasma frequency and the passband

$$
\Delta \omega=\frac{\omega_{p}}{2 Q_{p}} .
$$

Another important practical application of transverse plasma resonance is possibility its use for warming-up and diagnostics of plasma. If the quality of plasma resonator is great, then can be obtained the high levels of electrical fields on, and it means high energies of charge carriers. 


\section{$\S 8$. Kinetic capacity}

If we consider all components of current density in the conductor, then the Maxwell second equation can be written down:

$$
\operatorname{rot} \mathbf{H}=\sigma_{E} \mathbf{E}+\varepsilon \frac{\partial \mathbf{E}}{\partial t}+\frac{1}{L_{k}} \int \mathbf{E} d t,
$$

where $\sigma_{E}$ - conductivity of metal.

At the same time, the Maxwell first equation can be written down as follows:

$$
\operatorname{rot} \mathbf{E}=-\mu \frac{\partial \mathbf{H}}{\partial t}
$$

where $\mu$-magnetic permeability of medium. It is evident that equations (8.1) and (8.2) are asymmetrical.

To somewhat improve the symmetry of these equations are possible, introducing into equation (8.2) term linear for the magnetic field, that considers heat losses in the magnetic materials in the variable fields:

$$
\operatorname{rot} \mathbf{E}=-\sigma_{H} \mathbf{H}-\mu \frac{\partial \mathbf{H}}{\partial t}
$$

where $\sigma_{H}$ - conductivity of magnetic currents. But here there is no integral of such type, which is located in the right side of equation (8.1), in this equation. At the same time to us it is known that the atom, which possesses the magnetic moment $\mathrm{m}$, placed into the magnetic field, and which accomplishes in it precessional motion, has potential energy $U_{m}=-\mu \mathbf{m} \mathbf{H}$. Therefore potential energy can be accumulated not only in the electric fields, but also in the precessional motion of magnetic moments, which does not possess inertia. Similar case is located also in the mechanics, when the gyroscope, which precesses in the field of external gravitational forces, accumulates potential energy. Regarding mechanical precessional motion is also no inertial and immediately ceases after the removal of external forces. For example, if we from under the precessing gyroscope, which revolves in the field of the earth's gravity, rapidly remove support, thus it will begin to fall, preserving in the space the direction of its axis, which was at the moment, when support was removed. The same situation occurs also for the case of the precessing magnetic moment. Its precession is no inertial and ceases at the moment of removing the magnetic field.

Therefore it is possible to expect that with the description of the precessional motion of magnetic moment in the external magnetic field in the right side of relationship (8.3) can appear a term of the same type as in relationship (8.1). It will only stand $L_{k}$, i.e., instead $C_{k}$ the kinetic capacity, which characterizes that potential energy, which has the precessing magnetic moment in the magnetic field $[9,29,30]$ :

$$
\operatorname{rot} \mathbf{E}=-\sigma_{H} \mathbf{H}-\mu \frac{\partial \mathbf{H}}{\partial t}-\frac{1}{C_{k}} \int \mathbf{H} d t .
$$

For the first time this idea of the Maxwell first equation taking into account kinetic capacity was given in the work [10] 
Let us explain, can realize this case in practice, and that such in this case kinetic capacity. Resonance processes in the plasma and the dielectrics are characterized by the fact that in the process of fluctuations occurs the alternating conversion of electrostatic energy into the kinetic kinetic energy of charges and vice versa. This process can be named electro kinetic and all devices: lasers, masers, filters, etc. which use this process, can be named electrokinetic. At the same time there is another type of resonance - magnetic. If we use ourselves the existing ideas about the dependence of magnetic permeability on the frequency, then it is not difficult to show that this dependence is connected with the presence of magnetic resonance. In order to show this, let us examine the concrete example of ferromagnetic resonance. If we magnetize ferrite, after applying the stationary field $H_{0}$ in parallel to the axis $z$, the like to relation to the external variable field medium will come out as anisotropic magnetic material with the complex permeability in the form of tensor [31]

$$
\mu=\left(\begin{array}{lll}
\mu_{T}^{*}(\omega) & -i \alpha & 0 \\
i \alpha & \mu_{T}^{*}(\omega) & 0 \\
0 & 0 & \mu_{L}
\end{array}\right),
$$

where

$$
\mu_{T}^{*}(\omega)=1-\frac{\Omega|\gamma| M_{0}}{\mu_{0}\left(\omega^{2}-\Omega^{2}\right)}, \quad \alpha=\frac{\omega|\gamma| M_{0}}{\mu_{0}\left(\omega^{2}-\Omega^{2}\right)}, \quad \mu_{L}=1,
$$

moreover

$$
\Omega=\gamma H_{0}
$$

is natural frequency of precession and

$$
M_{0}=\mu_{0}(\mu-1) H_{0}
$$

is a magnetization of medium. Taking into account (4.4) and (4.5) for $\mu_{T} *(\omega)$, it is possible to write down

$$
\mu_{T}^{*}(\omega)=1-\frac{\Omega^{2}(\mu-1)}{\omega^{2}-\Omega^{2}} .
$$

That magnetic permeability of magnetic material depends on frequency, and can arise suspicions, that, as in the case with the plasma, here is some misunderstanding.

If we consider that the electromagnetic wave is propagated along the axis $x$ and there are components fields on $H_{y}$ and $H_{z}$, then in this case the Maxwell first equation will be written down:

$$
\operatorname{rot} \mathbf{E}=\frac{\partial \mathbf{E}_{Z}}{\partial x}=\mu_{0} \mu_{T} \frac{\partial \mathbf{H}_{y}}{\partial t} .
$$

Taking into account (8.6), we will obtain

$$
\operatorname{rot} \mathbf{E}=\mu_{0}\left[1-\frac{\Omega^{2}(\mu-1)}{\omega^{2}-\Omega^{2}}\right] \frac{\partial \mathbf{H}_{y}}{\partial t} .
$$

for the case $\omega>>\Omega$ we have 


$$
\operatorname{rot} \mathbf{E}=\mu_{0}\left[1-\frac{\Omega^{2}(\mu-1)}{\omega^{2}}\right] \frac{\partial \mathbf{H}_{y}}{\partial t}
$$

assuming $H_{y}=H_{y 0} \sin \omega t$ and taking into account that in this case

$$
\frac{\partial \mathbf{H}_{y}}{\partial t}=-\omega^{2} \int \mathbf{H}_{y} d t,
$$

we will obtain from $(8.7)$

$$
\operatorname{rot} \mathbf{E}=\mu_{0} \frac{\partial \mathbf{H}_{y}}{\partial t}+\mu_{0} \Omega^{2}(\mu-1) \int \mathbf{H}_{y} d t,
$$

or

$$
\operatorname{rot} \mathbf{E}=\mu_{0} \frac{\partial \mathbf{H}_{y}}{\partial t}+\frac{1}{C_{k}} \int \mathbf{H}_{y} d t .
$$

For the case $\omega<<\Omega$ we find

$$
\operatorname{rot} \mathbf{E}=\mu_{0} \mu \frac{\partial \mathbf{H}_{y}}{\partial t} .
$$

Value

$$
C_{k}=\frac{1}{\mu_{0} \Omega^{2}(\mu-1)},
$$

which is introduced in relationship (8.8), let us name kinetic capacity.

With which is connected existence of this parameter, and it's what physical sense? If the direction of magnetic moment does not coincide with the direction of external magnetic field, then the vector of this moment begins to precess around the vector of magnetic field with the frequency $\Omega$. The magnetic moment $\mathbf{m}$ possesses in this case potential energy $U_{m}=-\mathbf{m} \cdot \mathbf{B}$. This energy similar to energy of the charged capacitor is potential, because precessional motion, although is mechanical, however, it not inertia and instantly it does cease during the removal of magnetic field. However, with the presence of magnetic field precessional motion continues until the accumulated potential energy is spent, and the vector of magnetic moment will not become parallel to the vector of magnetic field.

The equivalent diagram of the case examined is given in Fig. 4. At the point $\omega$ $=\Omega$ occurs magnetic resonance, in this case $\mu_{\mathrm{T}}^{*}(\omega) \rightarrow-\infty$. The resonance frequency of macroscopic magnetic resonator, as can easily be seen of the equivalent diagram, also does not depend on the dimensions of line and is equal $\Omega$. Thus, the parameter

$$
\mu_{H} *(\omega)=\mu_{0}\left[1-\frac{\Omega^{2}(\mu-1)}{\omega^{2}-\Omega^{2}}\right]
$$

is not the frequency dependent magnetic permeability, but it is the combined parameter, including $\mu_{0}, \mu$ and $C_{k}$, which are included on in accordance with the equivalent diagram, depicted in Fig. 4.

Is not difficult to show that in this case there are three waves: electrical, magnetic and the wave, which carries potential energy, which is connected with the precession of magnetic moments around the vector $H_{0}$. For this reason such waves can be named electricmagneticpotentsial waves. Before the appearance of a work [9] 
in the electrodynamics this concept, as kinetic capacity it was not used, although this the real parameter has very intelligible physical interpretation.

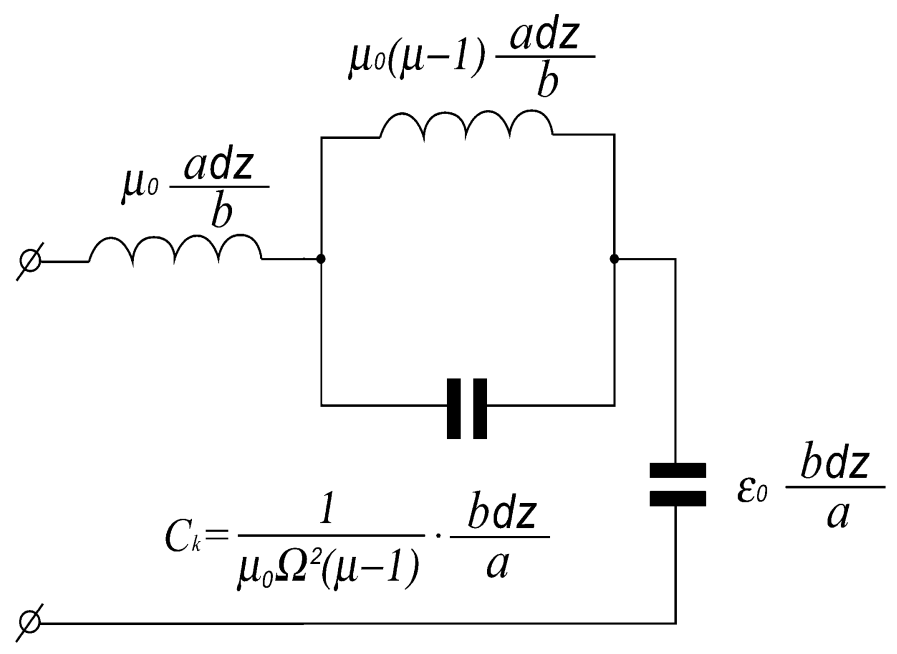

Fig. 4. Equivalent the schematic of the two-wire circuit of that filled with magnetic material

\section{§ 9. Dielectrics}

In the existing literature there are no indications that the kinetic inductance of charge carriers plays some role in the electrodynamic processes in the dielectrics. This not thus $[32,33]$. This parameter in the electrodynamics of dielectrics plays not less important role, than in the electrodynamics of conductors. Let us examine the simplest case, when oscillating processes in atoms or molecules of dielectric obey the law of mechanical oscillator [34]. Let us write down the equation of motion

$$
\left(\frac{\beta}{m}-\omega^{2}\right) \mathbf{r}_{m}=\frac{e}{m} \mathbf{E},
$$

where $\mathbf{r}_{m}$-deviation of charges from the position of equilibrium, $\beta$ - coefficient of elasticity, which characterizes the elastic electrical binding forces of charges in the atoms and the molecules. Introducing the resonance frequency of the bound charges

we obtain from $(9.1)$

$$
\omega_{0}=\frac{\beta}{m},
$$

$$
\mathbf{r}_{m}=-\frac{e \mathbf{E}}{m\left(\omega^{2}-\omega_{o}^{2}\right)} .
$$

Is evident that in relationship (9.2) as the parameter is present the natural vibration frequency, into which enters the mass of charge. This speaks, that the inertia properties of the being varied charges will influence oscillating processes in the atoms and the molecules.

Since the general current density on medium consists of the bias current and conduction current 


$$
\operatorname{rot} \mathbf{H}=\mathbf{j}_{\Sigma}=\varepsilon_{0} \frac{\partial \mathbf{E}}{\partial t}+n e \mathbf{v},
$$

that, finding the speed of charge carriers in the dielectric as the derivative of their displacement through the coordinate

$$
\mathbf{v}=\frac{\partial \mathbf{r}_{m}}{\partial t}=-\frac{e}{m\left(\omega^{2}-\omega_{o}^{2}\right)} \frac{\partial \mathbf{E}}{\partial t},
$$

from relationship (9.2) we find

$$
\operatorname{rot} \mathbf{H}=\mathbf{j}_{\Sigma}=\varepsilon_{0} \frac{\partial \mathbf{E}}{\partial t}-\frac{1}{L_{k d}\left(\omega^{2}-\omega_{0}^{2}\right)} \frac{\partial \mathbf{E}}{\partial t} .
$$

Let us note that the value

$$
L_{k d}=\frac{m}{n e^{2}}
$$

presents the kinetic inductance of the charges, entering the constitution of atom or molecules of dielectrics, when to consider charges free. Therefore relationship (9.3) it is possible to rewrite

$$
\operatorname{rot} \mathbf{H}=\mathbf{j}_{\Sigma}=\varepsilon_{0}\left(1-\frac{1}{\varepsilon_{0} L_{k d}\left(\omega^{2}-\omega_{0}^{2}\right)}\right) \frac{\partial \mathbf{E}}{\partial t} .
$$

Since the value

$$
\frac{1}{\varepsilon_{0} L_{k d}}=\omega_{p d}^{2}
$$

it represents the plasma frequency of charges in atoms and molecules of dielectric, if we consider these charges free, then relationship (9.4) takes the form:

$$
\operatorname{rot} \mathbf{H}=\mathbf{j}_{\Sigma}=\varepsilon_{0}\left(1-\frac{\omega^{2}{ }_{p d}}{\left(\omega^{2}-\omega_{0}^{2}\right)}\right) \frac{\partial \mathbf{E}}{\partial t} .
$$

To appears temptation to name the value

$$
\varepsilon^{*}(\omega)=\varepsilon_{0}\left(1-\frac{\omega^{2}{ }_{p d}}{\left(\omega^{2}-\omega_{0}^{2}\right)}\right)
$$

by the depending on the frequency dielectric constant of dielectric. But this, as in the case conductors, cannot be made, since this is the composite parameter, which includes now those not already three depending on the frequency of the parameter: the dielectric constant of vacuum, the natural frequency of atoms or molecules and plasma frequency for the charge carriers, entering their composition.

Let us examine two limiting cases:

1. If $\omega<<\omega_{0}$ then from (9.5) we obtain

$$
\operatorname{rot} \mathbf{H}=\mathbf{j}_{\Sigma}=\varepsilon_{0}\left(1+\frac{\omega_{p d}^{2}}{\omega_{0}^{2}}\right) \frac{\partial \mathbf{E}}{\partial t} .
$$


In this case the coefficient, confronting the derivative, does not depend on frequency, and it presents the static dielectric constant of dielectric. As we see, it depends on the natural frequency of oscillation of atoms or molecules and on plasma frequency. This result is intelligible. Frequency in this case proves to be such low that the charges manage to follow the field and their inertia properties do not influence electrodynamic processes. In this case the bracketed expression in the right side of relationship (9.7) presents the static dielectric constant of dielectric. As we see, it depends on the natural frequency of oscillation of atoms or molecules and on plasma frequency. Hence immediately we have a prescription for creating the dielectrics with the high dielectric constant. In order to reach this, should be in the assigned volume of space packed a maximum quantity of molecules with maximally soft connections between the charges inside molecule itself.

2. The case, when $\omega>>\omega_{0}$ is exponential. In this case

$$
\operatorname{rot} \mathbf{H}=\mathbf{j}_{\Sigma}=\varepsilon_{0}\left(1-\frac{\omega_{p d}^{2}}{\omega^{2}}\right) \frac{\partial \mathbf{E}}{\partial t},
$$

and dielectric became conductor (plasma), since the obtained relationship exactly coincides with the equation, which describes plasma.

One cannot fail to note the circumstance that in this case again nowhere was used this concept as polarization vector, but examination is carried out by the way of finding the real currents in the dielectrics on the basis of the equation of motion of charges in these media. In this case as the parameters are used the electrical characteristics of the media, which do not depend on frequency.

From relationship (9.5) is evident that in the case of fulfilling the equality $\omega=\omega_{0}$, the amplitude of fluctuations is equal to infinity. This indicates the presence of resonance at this point. The infinite amplitude of fluctuations occurs because of the fact that they were not considered losses in the resonance system, in this case its quality was equal to infinity. In a certain approximation it is possible to consider that lower than the point indicated we deal concerning the dielectric, whose dielectric constant is equal to its static value. Higher than this point we deal already actually concerning the metal, whose density of current carriers is equal to the density of atoms or molecules in the dielectric.

Now it is possible to examine the question of why dielectric prism decomposes polychromatic light into monochromatic components or why rainbow is formed. So that this phenomenon would occur, it is necessary to have the frequency dispersion of the phase speed of electromagnetic waves in the medium in question. If we to relationship (9.5) add the Maxwell first equation, then we will obtain:

$$
\begin{aligned}
& \operatorname{rot} \mathbf{E}=-\mu_{0} \frac{\partial \mathbf{H}}{\partial t} \\
& \operatorname{rot} \mathbf{H}=\varepsilon_{0}\left(1-\frac{\omega_{p d}^{2}}{\left(\omega^{2}-\omega_{0}^{2}\right)}\right) \frac{\partial \mathbf{E}}{\partial t},
\end{aligned}
$$

from where we immediately find the wave equation: 


$$
\nabla^{2} \mathbf{E}=\mu_{0} \varepsilon_{0}\left(1-\frac{\omega_{p d}^{2}}{\omega^{2}-\omega_{0}^{2}}\right) \frac{\partial^{2} \mathbf{E}}{\partial t^{2}}
$$

If one considers that

$$
\mu_{0} \varepsilon_{0}=\frac{1}{c^{2}},
$$

where $c$ - speed of light, then no longer will remain doubts about the fact that with the propagation of electromagnetic waves in the dielectrics the frequency dispersion of phase speed will be observed. In the formation of this dispersion it will participate immediately three, which do not depend on the frequency, physical quantities: the self-resonant frequency of atoms themselves or molecules, the plasma frequency of charges, if we consider it their free, and the dielectric constant of vacuum.

Now let us show, where it is possible to be mistaken, if with the solution of the examined problem of using a concept of polarization vector. Let us introduce this polarization vector

$$
\mathbf{P}=-\frac{n e^{2}}{m} \cdot \frac{1}{\left(\omega^{2}-\omega_{0}^{2}\right)} \mathbf{E} .
$$

Its dependence on the frequency, is connected with the presence of mass in the charges, entering the constitution of atom and molecules of dielectrics. The inertness of charges is not allowed for this vector, following the electric field, to reach that value, which it would have in the permanent fields. Since the electrical induction is determined by the relationship:

$$
\mathbf{D}=\varepsilon_{0} \mathbf{E}+\mathbf{P E}=\varepsilon_{0} \mathbf{E}-\frac{n e^{2}}{m} \cdot \frac{1}{\left(\omega^{2}-\omega_{0}^{2}\right)} \mathbf{E},
$$

that, introduced thus, it depends on frequency.

If this induction was introduced into the Maxwell second equation, then it signs the form:

$$
\operatorname{rot} \mathbf{H}=\mathbf{j}_{\Sigma}=\varepsilon_{0} \frac{\partial \mathbf{E}}{\partial t}+\frac{\partial \mathbf{P}}{\partial t}
$$

or

$$
\operatorname{rot} \mathbf{H}=\mathbf{j}_{\Sigma}=\varepsilon_{0} \frac{\partial \mathbf{E}}{\partial t}-\frac{n e^{2}}{m} \frac{1}{\left(\omega^{2}-\omega_{0}^{2}\right)} \frac{\partial \mathbf{E}}{\partial t},
$$

where $\mathbf{j}_{\Sigma}$ - the summed current, which flows through the model. In expression (9.9) the first member of right side presents bias current in the vacuum, and the second current, connected with the presence of bound charges in atoms or molecules of dielectric. In this expression again appeared the specific kinetic inductance of the charges, which participate in the oscillating process

$$
L_{k d}=\frac{m}{n e^{2}} .
$$

This kinetic inductance determines the inductance of bound charges. Taking into account this relationship (9.9) it is possible to rewrite 


$$
\operatorname{rot} \mathbf{H}=\mathbf{j}_{\Sigma}=\varepsilon_{0} \frac{\partial \mathbf{E}}{\partial t}-\frac{1}{L_{k d}} \frac{1}{\left(\omega^{2}-\omega_{0}^{2}\right)} \frac{\partial \mathbf{E}}{\partial t} .
$$

Obtained expression exactly coincides with relationship (9.3). Consequently, the eventual result of examination by both methods coincides, and there are no claims to the method from a mathematical point of view. But from a physical point of view, and especially in the part of the awarding to the parameter, introduced in accordance with relationship (9.8) of the designation of electrical induction, are large claims, which we discussed. Is certain, this not electrical induction, but the certain composite parameter. But, without having been dismantled at the essence of a question, all, until now, consider that the dielectric constant of dielectrics depends on frequency. In the essence, physically substantiated is the introduction to electrical induction in the dielectrics only in the static electric fields.

Let us show that the equivalent the schematic of dielectric presents the sequential resonant circuit, whose inductance is the kinetic inductance $L_{k d}$, and capacity is equal to the static dielectric constant of dielectric minus the capacity of the equal dielectric constant of vacuum. In this case outline itself proves to be that shunted by the capacity, equal to the specific dielectric constant of vacuum. For the proof of this let us examine the sequential oscillatory circuit, when the inductance $L$ and the capacity $C$ are connected in series.

The connection between the current $I_{C}$, which flows through the capacity $C$, and the voltage $U_{C}$, applied to it, is determined by the relationships:

$$
U_{C}=\frac{1}{C} \int I_{C} d t
$$

and

$$
I_{C}=C \frac{d U_{C}}{d t}
$$

This connection will be written down for the inductance:

and

$$
I_{L}=\frac{1}{L} \int U_{L} d t
$$

$$
U_{L}=L \frac{d I_{L}}{d t}
$$

If the current, which flows through the series circuit, changes according to the law $I=I_{0} \sin \omega t$, then a voltage drop across inductance and capacity they are determined by the relationships

$$
U_{L}=\omega L I_{0} \cos \omega t
$$

and

$$
U_{C}=-\frac{1}{\omega C} I_{0} \cos \omega t,
$$

and total voltage applied to the outline is equal 


$$
U_{\Sigma}=\left(\omega L-\frac{1}{\omega C}\right) I_{0} \cos \omega t .
$$

In this relationship the value, which stands in the brackets, presents the reactance of sequential resonant circuit, which depends on frequency. The voltages, generated on the capacity and the inductance, are located in the reversed phase, and, depending on frequency, outline can have the inductive, the weather capacitive reactance. At the point of resonance the summary reactance of outline is equal to zero.

It is obvious that the connection between the total voltage applied to the outline and the current, which flows through the outline, will be determined by the relationship

$$
I=-\frac{1}{\omega\left(\omega L-\frac{1}{\omega C}\right)} \frac{\partial U_{\Sigma}}{\partial t} .
$$

The resonance frequency of outline is determined by the relationship

$$
\omega_{0}=\frac{1}{\sqrt{L C}},
$$

therefore let us write down

$$
I=\frac{C}{\left(1-\frac{\omega^{2}}{\omega_{0}^{2}}\right)} \frac{\partial U_{\Sigma}}{\partial t} .
$$

Comparing this expression with relationship (9.10) it is not difficult to see that the sequential resonant circuit, which consists of the inductance $L$ and capacity $C$, it is possible to present to the capacity of in the form dependent on the frequency

$$
C(\omega)=\frac{C}{\left(1-\frac{\omega^{2}}{\omega_{0}^{2}}\right)} .
$$

This idea does not completely mean that the inductance is somewhere lost. Simply it enters into the resonance frequency of the outline $\omega_{0}$. Relationship (9.12) this altogether only the mathematical form of the record of relationship (9.11). Consequently, this is $C(\omega)$ the certain composite mathematical parameter, which is not the capacity of outline.

Relationship (9.11) can be rewritten and differently:

$$
I=-\frac{1}{L\left(\omega^{2}-\omega_{0}^{2}\right)} \frac{\partial U_{\Sigma}}{\partial t},
$$

and to consider that

$$
C(\omega)=-\frac{1}{L\left(\omega^{2}-\omega_{0}^{2}\right)} .
$$

Is certain, the parameter $C(\omega)$, introduced in accordance with relationships (9.13) and (9.14) no to capacity refers.

Let us examine relationship (9.12) for two limiting cases: 
1. When $\omega<<\omega_{0}$ we have

$$
I=C \frac{\partial U_{\Sigma}}{\partial t} .
$$

This result is intelligible, since at the low frequencies the reactance of the inductance, connected in series with the capacity, is considerably lower than the capacitive and it is possible not to consider it.

The equivalent the schematic of the dielectric, located between the planes of long line is shown in Fig. 5.

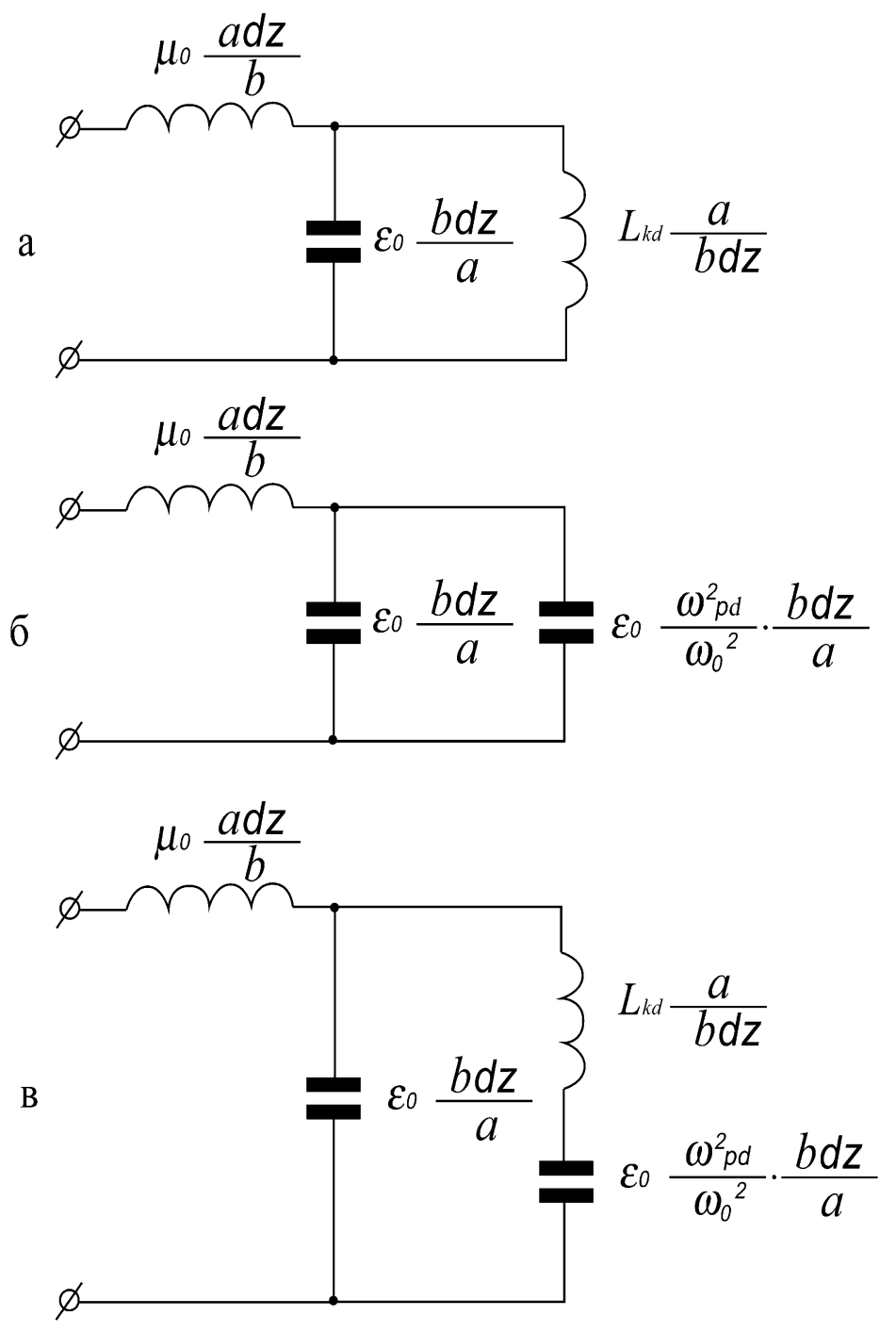

Fig. 5. a - the equivalent the schematic of the section of the line, filled with dielectric, for the case $\omega \gg>\omega_{0}$,

$\sigma$ - the equivalent the schematic of the section of line for the case $\omega<<\omega_{0}$,

В - the equivalent the schematic of the section of line for entire frequent cy band

2. For the case, when $\omega>>\omega_{0}$, we have 


$$
I=-\frac{1}{\omega^{2} L} \frac{\partial U_{\Sigma}}{\partial t}
$$

Taking into account that for the harmonic signal

we obtain from (9.15)

$$
\frac{\partial U_{\Sigma}}{\partial t}=-\omega^{2} \int U_{\Sigma} d t
$$

$$
I_{L}=\frac{1}{L} \int U_{\Sigma} d t
$$

In this case the reactance of capacity is considerably less than in inductance and chain has inductive reactance.

The carried out analysis speaks, that is in practice very difficult to distinguish the behavior of resonant circuits of the inductance or of the capacity. In order to understand the true composition of the chain being investigated it is necessary to remove the amplitude and phase response of this chain in the range of frequencies. In the case of resonant circuit this dependence will have the typical resonance nature, when on both sides resonance the nature of reactance is different. However, this does not mean that real circuit elements: capacity or inductance depend on frequency.

In Fig. 5 (a) and 5 (б) are shown two limiting cases. In the first case, when $\omega>>$ $\omega_{0}$, dielectric according to its properties corresponds to conductor, in the second case, when $\omega<<\omega_{0}$, it corresponds to the dielectric, which possesses the static dielectric constant $\varepsilon=\varepsilon_{0}\left(1+\frac{\omega_{p d}^{2}}{\omega_{0}^{2}}\right)$.

Thus, it is possible to make the conclusion that the introduction, the depending on the frequency dielectric constants of dielectrics, are physical and terminological error. If the discussion deals with the dielectric constant of dielectrics, with which the accumulation of potential energy is connected, then the discussion can deal only with the static permeability. And precisely this parameter as the constant, which does not depend on the frequency, enters into all relationships, which characterize the electrodynamic characteristics of dielectrics.

The most interesting results of applying such new approaches occur precisely for the dielectrics. In this case each connected pair of charges presents the separate unitary unit with its individual characteristics and its participation in the processes of interaction with the electromagnetic field (if we do not consider the connection between the separate pairs) strictly individually. Certainly, in the dielectrics not all dipoles have different characteristics, but there are different groups with similar characteristics, and each group of bound charges with the identical characteristics will resound at its frequency. Moreover the intensity of absorption, and in the excited state and emission, at this frequency will depend on a relative quantity of pairs of this type. Therefore the partial coefficients, which consider their statistical weight in this process, can be introduced. Furthermore, these processes will influence the anisotropy of the dielectric properties of molecules themselves, which have the specific electrical orientation in crystal lattice. By these circumstances is 
determined the variety of resonances and their intensities, which is observed in the dielectric media. The lines of absorption or emission, when there is an electric coupling between the separate groups of emitters, acquire even more complex structure. In this case the lines can be converted into the strips. Such individual approach to each separate type of the connected pairs of charges could not be realized within the framework earlier than the existing approaches.

Should be still one important circumstance, which did not up to now obtain proper estimation. With the examination of processes in the material media, which they are both conductors and dielectrics in all relationships together with the dielectric and magnetic constant figures the kinetic inductance of charges . This speaks, that the role of this parameter with the examination of processes in the material media has not less important role, than dielectric and magnetic constant. This is for the first time noted in a number the already mentioned sources, including in the recently published article [23]. 


\section{CHAPTER 3}

\section{NEW PROCEDURES AND CONCEPTS}

\section{$\S 10$. Surface kinetic inductance}

Until now, was considered that the kinetic inductance most effectively can appear only in the superconductors, and it was introduced by phenomenological method. But in the electrodynamics of conducting media, besides volumetric kinetic inductance, it is possible to introduce still and the concept of surface kinetic inductance, after enlarging by such means of the limit of the applicability of this term.

If there is a material medium, to boundary of which the plane electromagnetic wave will give, then some part of the energy of this wave passes into the material medium, and some is reflected. The process of the propagation of wave in medium itself is connected with its properties. For the introduction to surface kinetic inductance let us examine the case, when the frequency of the incident wave is considerably lower than the plasma [7].

Maxwell equations for the complex amplitudes fields on in this case they will be written down as follows:

$$
\begin{aligned}
& \operatorname{rot} \mathbf{E}=-i \omega \mu_{0} \mathbf{H}, \quad \operatorname{rot} \mathbf{H}=\mathbf{j} \\
& \operatorname{div} \mathbf{E}=0, \quad \operatorname{div} \mathbf{H}=0
\end{aligned}
$$

here and throughout the law of variation in the electromagnetic field is undertaken in the form $e^{i \omega t}$.

The surface resistance $R$ and the surface reactance $X$ are the numerical characteristics, which establish the connection between the tangential components of electrical and magnetic field on the surface, and those also determining the energy characteristics of interaction of surface with the electromagnetic field. The complex amplitudes of tangential components fields on the surface they are connected with the relationship

$$
E_{T}=\mathrm{ZH}_{T},
$$

from which it is not difficult to obtain the connection between the real fields on the surface

$$
\left|\tilde{E}_{T}\right|=\left|Z \| \tilde{H}_{T}\right| \cos (\omega t+\varphi)
$$

where

$$
Z=\frac{E_{x}(0)}{H_{y}(0)}=i \omega \mu_{0} \frac{1}{H_{y}(0)} \int_{0}^{\infty} H_{y}(z) d z
$$

the surface impedance of surface. It from this relation evident, that the module of surface impedance gives the ratio of the amplitudes of the tangential components of electrical and magnetic fields on the surface, and phase - phase displacement between them.

For establishing the connection $R$ and $X$ with the energy characteristics of surface layer let us take the single section of surface, for which are valid the Leonto- 
vich boundary conditions. Let us multiply first equation (10.4) by $\mathbf{H}^{*}$, and the second to $\mathbf{E}$ and let us piecemeal deduct one of another. After simple conversions we will obtain

$$
\operatorname{div} \mathbf{P}=-\frac{1}{2} \mathbf{j}^{*} \mathbf{E}-i \omega \frac{\mu_{0}|\mathbf{H}|^{2}}{2},
$$

where $\mathbf{P}=\frac{1}{2}\left[\mathbf{E} \times \mathbf{H}^{*}\right]$ - complex Pointing vector. Integrating (10.2) by the volume, which lies under the single area, after the conversion of left side from the formula of gauss let us find

$$
\int_{S} \mathbf{P} d \mathbf{S}=-\frac{1}{2} \int_{V} \mathbf{j}^{*} \mathbf{E} d S d z-i 2 \omega \int_{V} \frac{\mu_{0}|\mathbf{H}|^{2}}{4} d S d z
$$

where the integration is conducted according to the surface of the chosen area, and element of volume is recorded in the form $d S d z$.

We will consider that in the limits of the chosen area are small changes fields on in the tangential direction, and also that these fields become zero with $z \rightarrow \infty$.

In the surface integral in equation (10.3) is accepted $\mathbf{P} d \mathbf{S}=-\mathbf{P n} d S=-P_{n} d S$, where the vector $\vec{n}$ is directed into the depths of the medium in question. In relationship (10.3) are essential only tangential components $\mathbf{E}$ and $\mathbf{H}$, taking into account that

$$
\left[\mathbf{E}_{T} \times \mathbf{H}_{T}{ }^{*}\right]=Z\left|\mathbf{H}_{T}\right|^{2} \vec{n},
$$

this equation is reduced to the form:

$$
\frac{1}{2} Z\left|\mathbf{H}_{T}(0)\right|^{2}=\frac{1}{2} \int_{0}^{\infty} \mathbf{j}^{*} \mathbf{E} d z+i 2 \omega \int_{0}^{\infty} \frac{\mu_{0}|\mathbf{H}|^{2}}{4} d z
$$

After isolating the real part of this equality, we will obtain:

$$
P_{R}=\frac{1}{2} R\left|H_{T}(0)\right|^{2}=\operatorname{Re} \frac{1}{2} \int_{0}^{\infty} \mathbf{j}^{*} \mathbf{E} d z,
$$

where $P_{R}$ - average power of losses to the single square of surface.

Separating the imaginary part of equation (10.4), we find:

$$
P_{X}=\frac{1}{2} X\left|\mathbf{H}_{T}(0)\right|^{2}=\operatorname{Im} \frac{1}{2} \int_{0}^{\infty} \mathbf{j}^{*} \mathbf{E} d z+2 \omega \int_{0}^{\infty} \frac{\mu_{0}|\mathbf{H}|^{2}}{4} d z,
$$

where $P_{X}$ - average reactive power, which falls to the single square of surface.

Is evident that the reactive power consists of two members. The first of them represents the reactive power, connected with the kinetic energy of current carriers, and the second - gives the reactive power, connected with the presence on medium of magnetic field.

Boundary conditions:

$$
\mathbf{E}_{T}(0)=Z\left[\mathbf{H}_{T}(0) \times \mathbf{n}\right],
$$

where $Z=R+i X$, in connection with to real values fields $\tilde{E}_{T}$ and $\tilde{H}_{T}$ it is possible to write down in the form:

$$
\tilde{E}_{T}=R \tilde{H}_{T}+L \frac{d \tilde{H}}{d t}
$$


where $L=\frac{X}{\omega}$ - there is surface inductance of surface.

Now it is possible to introduce still and such new concepts as the kinetic and field surface of the inductance

$$
\begin{gathered}
L_{K}=\frac{1}{\omega\left|\mathbf{H}_{T}(0)\right|^{2}} \operatorname{Im} \int_{0}^{\infty} \mathbf{j}^{*} \mathbf{E} d z, \\
L_{\Pi}=\frac{1}{\left|\mathbf{H}_{T}(0)\right|^{2}} \int_{0}^{\infty}\left|\mathbf{H}_{T}\right|^{2} d z .
\end{gathered}
$$

These relationships are valid for the case of the arbitrary connection between the current and the field both in the normal metals and in the superconductors.

The examination of kinetic processes in the conductors and the dielectrics revealed one interesting special feature. If the charges are free, then in this system only collective fluctuations can exist, with which all charges, which participate in the oscillating process, are completely equal. They all are found in one and the same energy state and, if we do not consider loss, then the sum of kinetic and potential energy at any moment of time in them is identical. This conclusion is completely valid for the case of superconductors and cold plasma.

\section{$\S 11$. Electrical self-induction}

To the laws of self-induction should be carried those laws, which describe the reaction of such elements of radio-technical chains as capacity, inductance and resistance with the galvanic connection to them of the sources of current or voltage. These laws are the basis of the theory of electrical chains. The results of this theory can be postponed also by the electrodynamics of material media, since. such media can be represented in the form equivalent diagrams with the use of such elements.

The motion of charges in any chain, which force them to change their position, is connected with the energy consumption from the power sources. The processes of interaction of the power sources with such structures are regulated by the laws of self-induction.

Again let us refine very concept of self-induction. By self-induction we will understand the reaction of material structures with the constant parameters to the connection to them of the sources of voltage or current. to the self-induction let us carry also that case, when its parameters can change with the presence of the connected power source or the energy accumulated in the system. This self-induction we will call parametric. Subsequently we will use these concepts: as current generator and the voltage generator. By ideal voltage generator we will understand such source, which ensures on any load the lumped voltage, internal resistance in this generator equal to zero. By ideal current generator we will understand such source, which ensures in any load the assigned current, internal resistance in this generator equally to infinity. The ideal current generators and voltage in nature there does not exist, since both the current generators and the voltage generators have their internal resistance, which limits their possibilities. 
If we to one or the other network element connect the current generator or voltage, then opposition to a change in its initial state is the response reaction of this element and this opposition is always equal to the applied action, which corresponds to third Newton's law.

If at our disposal is located the capacity $C$, and this capacity is charged to a potential difference $U$, then the charge $Q$, accumulated in the capacity, is determined by the relationship:

$$
Q_{C, U}=C U \text {. }
$$

The charge $Q_{C, U}$, depending on the capacitance values of capacitor and from a voltage drop across it, we will call still the flow of electrical self-induction.

When the discussion deals with a change in the charge, determined by relationship (11.1), then this value can change with the method of changing the potential difference with a constant capacity, either with a change in capacity itself with a constant potential difference, or and that and other parameter simultaneously.

If capacitance value or voltage drop across it depend on time, then the current strength is determined by the relationship:

$$
I=\frac{d Q_{C, U}}{d t}=C \frac{\partial U}{\partial t}+U \frac{\partial C}{\partial t} .
$$

This expression determines the law of electrical self-induction. Thus, current in the circuit, which contains capacitor, can be obtained by two methods, changing voltage across capacitor with its constant capacity either changing capacity itself with constant voltage across capacitor, or to produce change in both parameters simultaneously.

For the case, when the capacity $C_{1}$ is constant, we obtain known expression for the current, which flows through the capacity:

$$
I=C_{1} \frac{\partial U}{\partial t},
$$

when changes capacity, and at it is supported the constant voltage $U_{1}$, we have:

$$
I=U_{1} \frac{\partial C}{\partial t} .
$$

This case to relate to the parametric electrical self-induction, since the presence of current is connected with a change in this parameter as capacity.

Let us examine the consequences, which escape from relationship (11.2).

If we to the capacity connect the dc generator $I_{0}$, then voltage on it will change according to the law:

$$
U=I_{0} t / C_{1} .
$$

Thus, the capacity, connected to the source of direct current, presents for it the effective resistance $[34,35]$

which linearly depends on time.

$$
R=t / C_{1},
$$

This is understandable from a physical point of view, since in order to charge capacity, source must expend energy. 
The power, output by current source, is determined in this case by the relationship:

$$
P(t)=\frac{I_{0}^{2} t}{C_{1}} .
$$

The energy, accumulated by capacity in the time $t$, we will obtain, after integrating relationship (11.6) with respect to the time:

$$
W_{C}=\frac{I_{0}^{2} t^{2}}{2 C_{1}} .
$$

Substituting here the value of current from relationship (11.4), we obtain the dependence of the value of the accumulated in the capacity energy from the instantaneous value of voltage on it:

$$
W_{C}=\frac{1}{2} C_{1} U^{2} .
$$

Using for the case examined a concept of the flow of the electrical induction

$$
\Phi_{U}=C_{1} U=Q(U)
$$

and using relationship (11.2), we obtain

$$
I_{0}=\frac{d \Phi_{U}}{d t}=\frac{\partial Q(U)}{\partial t}
$$

i.e., if we to a constant capacity connect the source of direct current, then the current strength will be equal to the derivative of the flow of capacitive induction on the time.

Now we will support at the capacity constant voltage $U_{1}$, and change capacity itself, then

$$
I=U_{1} \frac{\partial C}{\partial t}
$$

It is evident that the value

$$
R_{C}=\left(\frac{\partial C}{\partial t}\right)^{-1}
$$

plays the role of the effective resistance [34,35]. This result is also physically intelligible, since. with an increase in the capacitance increases the energy accumulated in it, and thus, capacity extracts in the voltage source energy, presenting for it resistive load. The power, expended in this case by source, is determined by the relationship:

$$
P(t)=\frac{\partial C}{\partial t} U_{1}^{2} .
$$

From relationship (11.11) is evident that depending on the sign of derivative the expendable power can have different signs. When the derived positive, expendable power goes for the accomplishment of external work. If derived negative, then external source accomplishes work, charging capacity.

Again, introducing concept the flow of the electrical induction

$$
\Phi_{C}=C U_{1}=Q(C)
$$


we obtain

$$
I=\frac{\partial \Phi_{C}}{\partial t} .
$$

Relationships (11.8) and (11.12) indicate that regardless of the fact, how changes the flow of electrical self-induction (charge), its time derivative is always equal to current.

Let us examine one additional process, which earlier the laws of induction did not include, however, it falls under for our extended determination of this concept. From relationship (11.7) it is evident that if the charge, left constant (we will call this regime the regime of the frozen electric flux), then voltage on the capacity can be changed by its change. In this case the relationship will be carried out:

$$
C U=C_{0} U_{0}=\text { const }
$$

where $C, U$ - instantaneous values, $C_{0}, U_{0}$ - initial values of these parameters, which occur with turning off from the capacity of the power source.

The voltage on the capacity and the energy, accumulated in it, will be in this case determined by the relationships:

$$
\begin{gathered}
U=\frac{C_{0} U_{0}}{C}, \\
W_{C}=\frac{1}{2} \frac{\left(C_{0} U_{0}\right)^{2}}{C} .
\end{gathered}
$$

It is natural that this process of self-induction can be connected only with a change in capacity itself, and therefore it falls under for the determination of parametric self-induction.

Thus, are located three relationships (11.8), (11.12) and (11.13), which determine the processes of electrical self-induction. We will call their rules of the electric flux. Relationship (11.8) determines the electrical self-induction, during which there are no changes in the capacity, and therefore this self-induction can be named simply electrical self-induction. Relationships (11.3) and (11.9)-(11.11) assume the presence of changes in the capacity; therefore the processes, which correspond by these relationships, we will call electrical parametric self-induction.

\section{$\S 12$. Current self-induction}

Let us now move on to the examination of the processes, proceeding in the inductance. Let us introduce the concept of the flow of the current self-induction

$$
\Phi_{L, I}=L I \text {. }
$$

If inductance is shortened outed, and made from the material, which does not have effective resistance, for example from the superconductor, then

$$
\Phi_{L, I}=L_{1} I_{1}=\text { const },
$$

where $L_{1}, I_{1}$ - initial values of these parameters, which are located at the moment of the short circuit of inductance with the presence in it of current. This regime we will call the regime of the frozen flow $[34,35]]$. In this case the relationship is fulfilled: 


$$
I=\frac{I_{1} L_{1}}{L}
$$

where $I, L$ - instantaneous values of the corresponding parameters.

In flow regime examined of current induction remains constant, however, in connection with the fact that current in the inductance it can change with its change, this process falls under for the determination of parametric self-induction. The energy, accumulated in the inductance, in this case will be determined by the relationship

$$
W_{L}=\frac{1}{2} \frac{\left(L_{1} I_{1}\right)^{2}}{L}=\frac{1}{2} \frac{(\text { const })^{2}}{L} .
$$

Voltage on the inductance is equal to the derivative of the flow of current induction on the time:

$$
U=\frac{d \Phi_{L, I}}{d t}=L \frac{\partial I}{\partial t}+I \frac{\partial L}{\partial t} .
$$

Let us examine the case, when the inductance is constant.

$$
U=L_{1} \frac{\partial I}{\partial t}
$$

designating $\Phi_{I}=L_{1} I$, we obtain $U=\frac{d \Phi_{I}}{d t}$. After integrating expression (12.2) on the time, we will obtain:

$$
I=\frac{U t}{L_{1}} .
$$

Thus, the capacity, connected to the source of direct current, presents for it the effective resistance $[34,35]$

$$
R=\frac{L_{1}}{t},
$$

which decreases inversely proportional to time.

The power, expended in this case by source, is determined by the relationship:

$$
P(t)=\frac{U^{2} t}{L_{1}} .
$$

This power linearly depends on time. After integrating relationship (12.5) on the time, we will obtain the energy, accumulated in the inductance

$$
W_{L}=\frac{1}{2} \frac{U^{2} t^{2}}{L_{1}} \text {. }
$$

After substituting into expression (12.6) the value of voltage from relationship (12.3), we obtain:

$$
W_{L}=\frac{1}{2} L_{1} I^{2}
$$

This energy can be returned from the inductance into the external circuit, if we open inductance from the power source and to connect effective resistance to it.

Now let us examine the case, when the current $I_{1}$, which flows through the inductance, is constant, and inductance itself can change. In this case we obtain the relationship 
Thus, the value

$$
U=I_{1} \frac{\partial L}{\partial t} .
$$

$$
R(t)=\frac{\partial L}{\partial t}
$$

plays the role of the effective resistance $[34,35]$. As in the case the electric flux, effective resistance can be (depending on the sign of derivative) both positive and negative. This means that the inductance can how derive energy from without, so also return it into the external circuits. Introducing the designation $\Phi_{L}=L I_{1}$ and, taking into account (12.7), we obtain

$$
U=\frac{d \Phi_{L}}{d t} .
$$

The relationship (12.1), (12.6), (12.9) we will call the rules of current selfinduction, or the flow rules of current self-induction. From relationships (12.6) and (12.9) it is evident that, as in the case with the electric flux, the method of changing the flow does not influence eventual result, and its time derivative is always equal to the applied potential difference. Relationship (12.6) determines the current selfinduction, during which there are no changes in the inductance, and therefore it can be named simply current self-induction. Relationships (12.7), (12.8) assume the presence of changes in the inductance; therefore we will call such processes current parametric self-induction.

The law of the frozen current (12.1) leads to phenomenon of magnetic potential pit on superconductive rings unknown earlier.

Assume that in two coaxially located superconductive rings the currents are frozen; moreover current in the lower ring is considerably more than in the upper. In accordance with Biot-Savart law the magnetic induction of lower ring on the axis in the plane of upper ring takes the form:

$$
B=\frac{\mu_{0} I_{1} R_{1}^{2}}{2\left(R_{1}^{2}+z_{0}^{2}\right)^{3 / 2}},
$$

where $\mu_{0}$ - the magnetic constant, $z_{0}$ - the distance between the rings, $R_{1}$ - the diameter of lower ring, $I_{1}$ - the current, frozen in the lower ring.

If a radius of upper ring composes $R_{2}$, its diameter considerably smaller than a radius of lower ring, then the magnetic flux, created by lower ring and which penetrates upper ring, will comprise

$$
\Phi_{2} \cong \frac{\mu_{0} I_{1} R_{1}^{2} R_{2}^{2}}{2\left(R_{1}^{2}+z_{0}^{2}\right)^{3 / 2}} .
$$

We will consider that the distance between the rings is considerably more than the diameter of lower ring, then

$$
\Phi_{2} \cong \mu_{0} I_{1} R_{1}^{2} R_{2}^{2} /\left(2 z_{0}^{3}\right) .
$$

If in the upper ring current is frozen $I_{2}$, that the flow is connected with it

$$
\Phi_{2}=L_{2} I_{2},
$$

where $L_{2}$ - the inductance of upper ring. 
Let us assume that in the initial position of rings direction of flow in them coincides, and rings are attracted. During lowering of lower ring the currents of induction will compensate currents in the upper ring, and current in it will reach the zero value, when the equality will be carried out

$$
\Phi_{2} \cong \frac{\mu_{0} I_{1} R_{1}^{2} R_{2}^{2}}{2 z_{0}^{3}} .
$$

In this case the distance between the rings will comprise

$$
z_{0}=\left(\frac{\mu_{0} I_{1} R_{1}^{2} R_{2}^{2}}{2 \Phi_{2}}\right)^{1 / 3}=\left(\frac{\mu_{0} I_{1} R_{1}^{2} R_{2}^{2}}{2 L_{2} I_{2}}\right)^{1 / 3} .
$$

With obtaining of this relationship the mutual inductance of rings is not taken into account, since. the distance between the rings is great, and it was also considered that the current $I_{1}$ practically does not change with the approximation of upper ring to lower.

At point $z_{0}$ the currents of induction completely compensate the current, frozen in the upper ring, therefore, this ring will no longer interact with the lower ring. With further rapprochement of rings the current of induction in the upper ring changes its initial direction and it will be opposite in the direction of flow in the lower ring, and, therefore, it will begin from it to be repulsed. But if rings will be moved away from each other, then in the upper ring they will arise current, the coinciding in the direction with the currents in the lower ring, and rings will be attracted. Thus, the position of upper ring at point $z_{0}$ is potential well. Such properties of the superconductive suspensions in the literary sources are not described.

This analysis of the possibility in principle of obtaining potential well on the superconductive rings with the frozen currents does not consider the mutual inductance $M(z)$ of rings, which for two circular outlines of a radius $R_{1}$ and $R_{2}$ with the common axis and by the distance $z$ between them is expressed as the complete elliptic integrals of the 1 st $K(k)$ and 2 nd $E(k)$ kind:

$$
\begin{gathered}
M(z)=\mu_{0} f(k(z)) \sqrt{R_{1} R_{2}}, \\
f(k)=\left(\frac{2}{k}-k\right) K(k)-\frac{2}{k} E(k)=\frac{2}{k}\left[\left(1-\frac{k^{2}}{2}\right) K(k)-E(k)\right], k^{2}=\frac{4 R_{1} R_{2}}{z^{2}+\left(R_{1}+R_{2}\right)^{2}}, \\
K(k)=\int_{0}^{\frac{\pi}{2}} \frac{d \theta}{\sqrt{1-k^{2} \sin ^{2} \theta}}, E(k)=\int_{0}^{\frac{\pi}{2}} \sqrt{1-k^{2} \sin ^{2} \theta} d \theta .
\end{gathered}
$$

Subsequently for the calculations their own inductances of rings with radii of the section of wires will be used also $r_{1}$ and $r_{2}$ :

$$
L_{10}=\mu_{0} R_{1}\left(\ln \frac{8 R_{1}}{r_{1}}-1.75\right), L_{20}=\mu_{0} R_{2}\left(\ln \frac{8 R_{2}}{r_{2}}-1.75\right) .
$$

Let us freeze currents $I_{10}, I_{20}$ in two secluded superconductive rings with its own inductances $L_{10}, L_{20}$. It will arrange these rings coaxially at a great distance so that the directions of flow in them again would coincide and let us begin 
to draw together them. Then we have relationships, which express the law of conservation of the frozen flows in the superconductive rings:

$$
L_{10} I_{1}(z)+M(z) I_{2}(z)=L_{10} I_{10} ; \quad L_{20} I_{2}(z)+M(z) I_{1}(z)=L_{20} I_{20},
$$

From them we will obtain relationships for the currents:

$$
I_{1}(z)=\frac{I_{10} L_{10} L_{20}-I_{20} L_{20} M(z)}{L_{10} L_{20}-M^{2}(z)} ; \quad I_{2}(z)=\frac{I_{20} L_{10} L_{20}-I_{10} L_{10} M(z)}{L_{10} L_{20}-M^{2}(z)}
$$

From these relationships we will obtain the value of the mutual inductance, with which the current in the lower and upper rings is equal to zero:

$$
M_{1}\left(z_{1}\right)=L_{1} \frac{I_{10}}{I_{20}} ; \quad M_{2}\left(z_{2}\right)=L_{2} \frac{I_{20}}{I_{10}} .
$$

Thus, assigning different initial values of inductances and currents frozen in them, it is possible to assign current zeros in that or other ring with different distances between them.

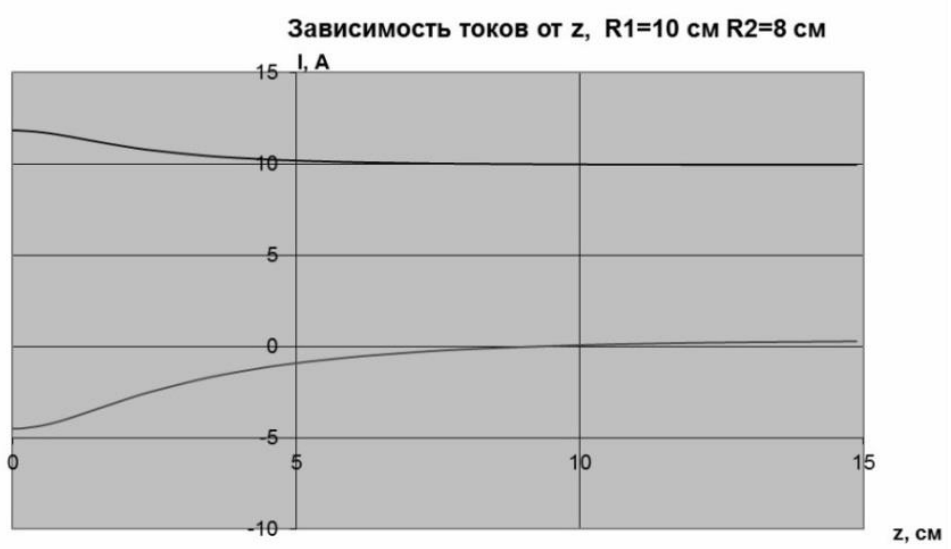

Fig. 6. Graphs of currents in the lower ring (upper curve) and in the upper ring (lower curve) with the initial values of current strength in the lower ring - $10 \mathrm{~A}$ and the value of current strength in the upper ring -2 a.

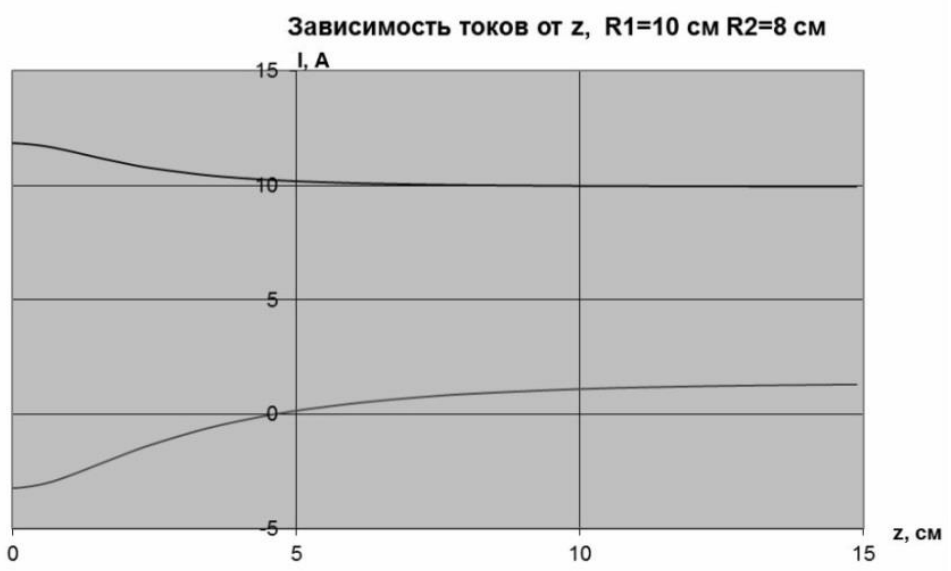

Fig. 7. Graphs of currents in the lower ring (upper curve) and in the upper ring (lower curve) with the initial values of current strength in the lower ring - $10 \mathrm{~A}$ and the value of current strength in the upper ring -5 a. 
In Fig. 6 and Fig. 7 are depicted the drawings calculated by (12.11) formulas $I_{1}(z)$, also, $I_{2}(z)$ with different initial values of currents in the rings of radii $R_{1}=10 \mathrm{~cm}, R_{2}=8 \mathrm{~cm}$ (radii of the section of wires $a_{1}=a_{2}=0,1 \mathrm{~cm}$ ). It is evident that at the specific distance from the lower ring the current in the upper reverses the sign. They coincide at the great distance of direction of flow in the rings, and they are attracted. They are opposite on - smaller than the direction, and they are repulsed. Consequently, the point, at which the current in the upper ring reverses the sign, is a coordinate of potential well.

The idea of the creation of potential well on the superconductive rings belongs to the authors of monograph, and (12.11) are obtained by Ivan Fedorovich Gorin - by a participant in the scientific forum movement fors the revival of the domestic science ( http://www.forum.za-nauku.ru/index.php/board,6.0.html ).

A participant in the same forum N. A. Drobyshev represented the solution of specific objectives, which we give below without the notes.

Task: The axes of two rings from the superconductive circular conductors coincide and are directed lengthwise $O z$, in this case reference point $O$ combined with the center of the first ring. To find currents in the rings and force of interaction of rings depending on the coordinate of the center of the second ring $z$, if with $z=z_{0}$ current in the second ring is absent, and in the first it is equal $I$. Radii of rings $r_{1} \quad r_{2}$ are considerably more than radii $a_{1}$ and $a_{2}$ the section of wires.

Answer: Current in the first ring

$$
I_{1}(z)=I \frac{b_{1} b_{2}-4 f\left(\kappa_{0}\right) f(\kappa)}{b_{1} b_{2}-4[f(\kappa)]^{2}}
$$

and the secondly -

$$
I_{2}(z)=2 I b_{1} \sqrt{\frac{r_{1}}{r_{2}}} \frac{f\left(\kappa_{0}\right)-f(\kappa)}{b_{1} b_{2}-4[f(\kappa)]^{2}}
$$

the force of interaction of the rings

$$
F(z)=\mu_{0} I^{2} g(\kappa) \frac{\left[b_{1} b_{2}-4 f\left(\kappa_{0}\right) f(\kappa)\right]\left[f\left(\kappa_{0}\right)-f(\kappa)\right]}{\left[b_{1} b_{2}-4[f(\kappa)]^{2}\right]^{2}} \frac{b_{1}|z|}{r_{2}}
$$

Here $\mu_{0}$ - magnetic constant, $b_{1}=\ln \left(8 r_{1} / a_{1}\right)-2$ and $b_{2}=\ln \left(8 r_{2} / a_{2}\right)-2$, A $\kappa=\kappa(z)=2 \sqrt{\frac{r_{1} r_{2}}{\left(r_{1}+r_{2}\right)^{2}+z^{2}}}, \quad \kappa_{0}=\kappa\left(z_{0}\right)=2 \sqrt{\frac{r_{1} r_{2}}{\left(r_{1}+r_{2}\right)^{2}+z_{0}^{2}}}$.

In the expressions (1)-(3) the functions figure

$$
f(\kappa)=\frac{1}{\kappa}\left[\left(1-\kappa^{2} / 2\right) K(\kappa)-E(\kappa)\right], \quad g(\kappa)=\kappa\left[K(\kappa)-\frac{1-\kappa^{2} / 2}{1-\kappa^{2}} E(\kappa)\right],
$$

where $K(\kappa)$ and $E(\kappa)$ - the complete elliptic integrals of the 1st and 2nd kind:

$K(\kappa)=\int_{0}^{\pi / 2} \frac{d \theta}{\sqrt{1-\kappa^{2} \sin ^{2} \theta}}, \quad E(\kappa)=\int_{0}^{\pi / 2} \sqrt{1-\kappa^{2} \sin ^{2} \theta} d \theta$.

As an example let us take the following radii of rings: $r_{1}=10 \mathrm{~cm}$ and $r_{2}=8$ $\mathrm{cm}$, and radii of the section of wires $-a_{1}=a_{2}=0,1 \mathrm{~cm}$. Let with the distance 
$z_{0}=5 \mathrm{~cm}$ between the centers of rings the current strength in the first ring compose $I=10 \mathrm{~A}$, and the secondly current is absent. Dependences $I_{1}(z), I_{2}(z)$ and $F(z)$, calculated by formulas (1)-(3), are depicted on the graphs. Current in the first ring $I_{1}$ is equal $I=10$ A with $z= \pm z_{0}$ and $z \rightarrow \pm \infty$. Current in the second ring $I_{2}$ is turned into zero with $z= \pm z_{0}$.
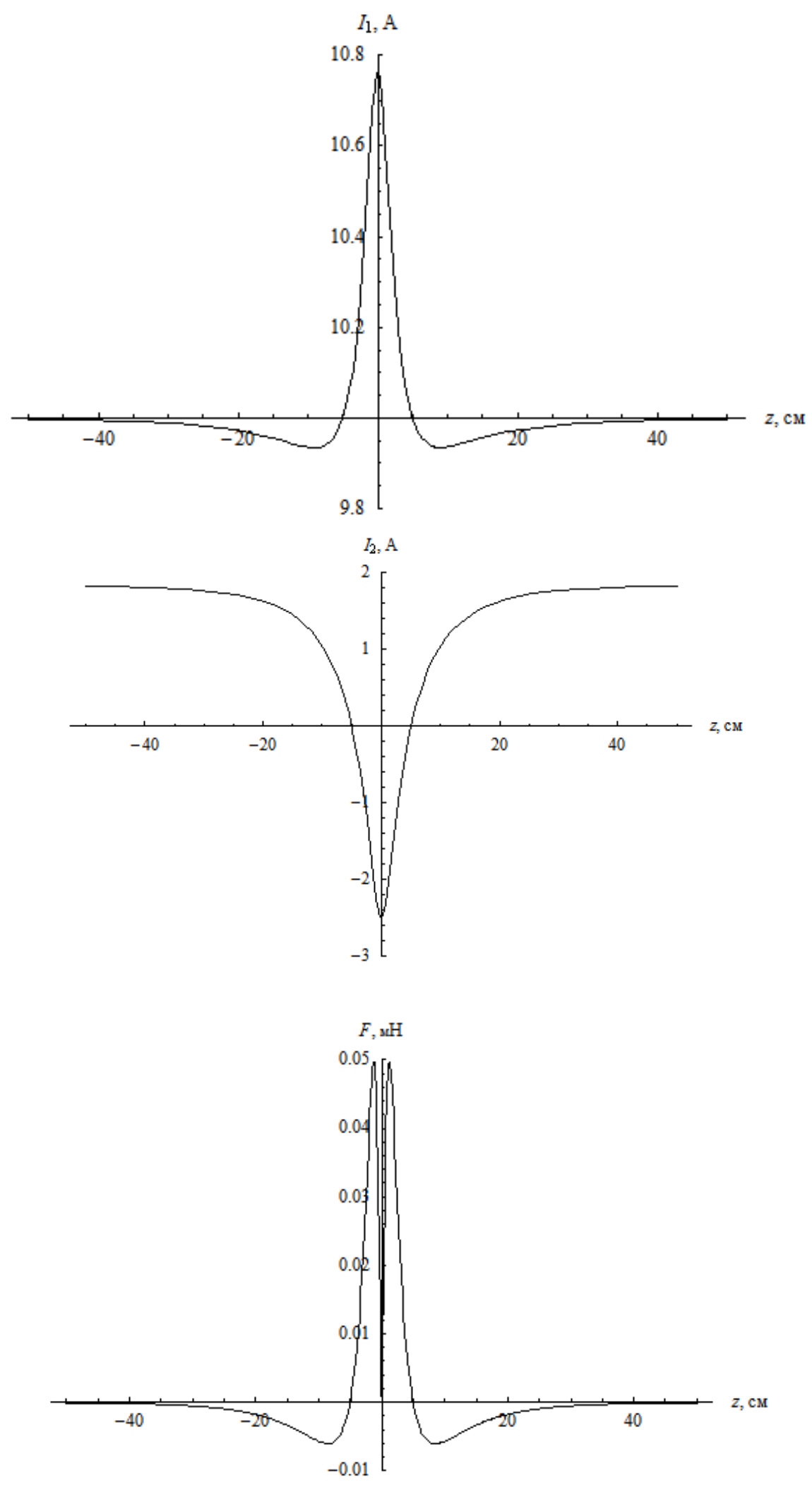
Positive values $I_{2}$ correspond to identical directions of flow in the rings, and negative- opposite. Finally, the positive values of force $F$ correspond to the repulsion of rings, and negative - to attraction. It is obvious that with $z= \pm z_{0}$ it becomes zero, in this case the rings are located in the position of stable equilibrium. Force $F$ is absent also with $z=0$ (when the planes of rings coincide), but in this case equilibrium - is unstable.

This fact testifies about the large usefulness of the collaboration of scientists on scientific the forum indicated, when some propose idea, and others find its mathematical embodiment.

\section{$\S 13$. New method of obtaining the wave equation, the potential and kinetic flows of charges}

The processes, examined in two previous paragraphs, concern chains with the lumped parameters, when the distribution of potential differences and currents in the elements examined can be considered uniform. However, there are chains, for example the long lines, into which potential differences and currents are not threedimensional uniform. These processes are described by the wave equations, which can be obtained from Maxwell equations or with the aid of the telegraphic equations, but physics of phenomenon itself in these processes to us is not clear [36].

Let us assume that linear capacity and inductance of this line are equal $C_{0}, L_{0}$. If we to this line connect the dc power supply $U_{1}$, then its front will be extended in the line some by the speed $v$, and the moving coordinate of this front will be determined by the relationship $z=v t$. In this case the total quantity of the charged capacity and the value of the summary inductance, along which it flows current, calculated from the beginning lines to the location of the front of voltage, will change according to the law [36]:

$$
\begin{aligned}
& C(t)=z C_{0}=v t C_{0}, \\
& L(t)=z L_{0}=v t L_{0} .
\end{aligned}
$$

The source of voltage $U_{1}$ will in this case charge the being increased capacity of line, for which from the source to the charged line in accordance with relationship (11.9) must leak the current:

$$
I_{1}=U_{1} \frac{\partial C(t)}{\partial t}=v U_{1} C_{0}
$$

This current there will be the leak through the conductors of line, that possess inductance. But, since the inductance of line in connection with the motion of the front of voltage, also increases, in accordance with relationship (12.7), on it will be observed a voltage drop:

$$
U=I_{1} \frac{\partial L(t)}{\partial t}=v I_{1} L_{0}=v^{2} U_{1} C_{0} L_{0} .
$$

But a voltage drop across the conductors of line in the absolute value is equal to the voltage, applied to its entrance; therefore in the last expression should be placed $U=U_{1}$. We immediately find taking this into account that the rate of the 
motion of the front of voltage with the assigned linear parameters and when, on, the incoming line of constant voltage $U_{1}$ is present, must compose

$$
v=\frac{1}{\sqrt{L_{0} C_{0}}}
$$

this expression corresponds to the signal velocity in line itself. Consequently, if we to the infinitely long line connect the voltage source, then in it will occur the expansion of electrical fields on and the currents, which fill line with energy, and the speed of the front of constant voltage and current will be equal to the velocity of propagation of electromagnetic vibrations in this line. This wave we will call electriccurent wave. It is interesting to note that the obtained result does not depend on the form of the function $U$, i.e., to the line can be connected both the dc power supply and the source, whose voltage changes according to any law. In all these cases the value of the local value of voltage on incoming line will be extended along it with the speed, which follows from relationship (13.2). This result could be, until now, obtained only by the method of solution of wave equation, but in this case he indicates the physical cause for this propagation, and it gives the physical picture of process itself. Examination shows that very process of propagation is connected with the energy processes of the filling of line with electrical and current energy. This process occurs in such a way that the wave front, being extended with the speed $v$, leaves after itself the line, charged to a potential difference $U_{1}$, which corresponds to the filling of line with electrostatic electric field energy. However, in the section of line from the voltage source also to the wave front flows the current $I_{1}$, which corresponds to the filling of line in this section with energy, which is connected with the motion of the charges along the conductors of line, which possess inductance.

The current strength in the line can be obtained, after substituting the values of the velocity of propagation of the wave front, determined by relationship (13.2), into relationship (13.1). After making this substitution, we will obtain

$$
I_{1}=U_{1} \sqrt{\frac{C_{0}}{L_{0}}}
$$

where $Z=\sqrt{\frac{L_{0}}{C_{0}}}$ - line characteristic.

In this case

So accurately

$$
U_{1}=I \frac{\partial L}{\partial t}=\frac{d \Phi_{L}}{d t}
$$

$$
I_{1}=U_{1} \frac{\partial C}{\partial t}=\frac{d \Phi_{C}}{d t} .
$$

It is evident that the flow rules both for the electrical and for the current selfinduction are observed also in this case.

Thus, the processes of the propagation of a potential difference along the conductors of long line and current in it are connected and mutually supplementing each 
other, and to exist without each other they do not can. This process can be called electriccurent spontaneous parametric self-induction. This name connected with the fact that flow expansion they occur arbitrarily and characterizes the rate of the process of the filling of line with energy. From the aforesaid the connection between the energy processes and the velocity of propagation of the wave fronts in the long lines becomes clear. Since with the emission of electromagnetic waves the free space is also transmission line, similar laws must characterize propagation in this space.

That will be, if we in the considered case as one of the conductors of long line take spiral, or to as is customary call, long solenoid. Obviously, in this case the velocity of propagation of the front of voltage in this line will decrease, since the linear inductance of line will increase. This propagation will accompany the process of the propagation not only of external with respect to the solenoid fields on and currents, but both the process of the propagation of magnetic flux inside the solenoid itself and the velocity of propagation of this flow will be equal to the velocity of propagation of electromagnetic wave in line itself.

Knowing current and voltage in the line, it is possible to calculate the specific energy, concluded in the linear capacity and the inductance of line. These energies will be determined by the relationships:

$$
\begin{gathered}
W_{C}=\frac{1}{2} C_{0} U_{1}^{2}, \\
W_{L}=\frac{1}{2} L_{0} I_{1}^{2} .
\end{gathered}
$$

It is not difficult to see that $W_{C}=W_{L}$.

Now let us discuss a question about the duration of the front of electriccurent wave and about which space will occupy this front in line itself. Answer to the first question is determined by the properties of the very voltage source, since. local derivative $\frac{\partial U}{\partial t}$ at incoming line depends on transient processes in the source itself and in that device, with the aid of which this source is connected to the line. If the process of establishing the voltage on incoming line will last some time $\Delta t$, then in the line it will engage section with the length $v \Delta t$. If we to the line exert the voltage, which is changed with the time according to the law $U(t)$, then the same value of function will be observed at any point of the line at a distance $z$ beginning with the delay $t=\frac{z}{v}$. Thus, the function

$$
U(t, z)=U\left(t-\frac{z}{v}\right)
$$

can be named propagation function, since. it establishes the connection between the local temporary and three-dimensional values of function in the line. Long line is the device, which converts local derivative voltage on the time on incoming line into the gradients in line itself. On the basis propagation function (13.5) it is possible to establish the connection between the local and gradients in the long line. It is obvious that 


$$
\frac{\partial U(z)}{\partial z}=\frac{1}{v} \frac{\partial U(t)}{\partial t} .
$$

Is important to note that very process of propagation in this case is obliged to the natural expansion of electric field and current in the line, and it is subordinated to the rules of parametric self-induction. In the second place, for solving the wave equations of the long lines

$$
\begin{aligned}
& \frac{\partial^{2} U}{\partial z^{2}}=\frac{1}{v^{2}} \frac{\partial^{2} U}{\partial t^{2}} \\
& \frac{\partial^{2} I}{\partial z^{2}}=\frac{1}{v^{2}} \frac{\partial^{2} I}{\partial t^{2}}
\end{aligned},
$$

obtained from the telegraphic equations

$$
\begin{aligned}
& \frac{\partial U}{\partial z}=-L \frac{\partial I}{\partial t} \\
& \frac{\partial I}{\partial z}=-C \frac{\partial U}{\partial t},
\end{aligned}
$$

the knowledge second derivative voltages and currents is required.

But what is to be done, if to incoming line is supplied voltage, whose second derivative is equal to zero (case, when the voltage of source it does change according to the linear law)? Answer to this question equation (13.6) they do not give. The utilized method gives answer also to this question.

With the examination of processes in the long line figured such concepts as linear capacity and inductance, and also currents and voltage in the line. However, in the electrodynamics, based on Maxwell equations, there are no such concepts as capacity and inductance, and there are concepts of the electrical and magnetic permeability of medium. In the carried out examination such concepts as electrical and magnetic fields also was absent. Let us show how to pass from such categories as linear inductance and capacity, current and voltage in the line to such concepts as dielectric and magnetic constant, and also electrical and magnetic field. For this let us take the simplest construction of line, located in the vacuum, as shown in Fig. 2 We will consider that $b>>a$ and edge effects it is possible not to consider. Then the following connection will exist between the linear parameters of line and the magnetic and dielectric constants:

$$
\begin{aligned}
L_{0} & =\mu_{0} \frac{a}{b}, \\
C_{0} & =\varepsilon_{0} \frac{b}{a},
\end{aligned}
$$

where $\mu_{0}, \varepsilon_{0}$ - dielectric and magnetic constant of vacuum.

The phase speed in this line will be determined by the relationship:

$$
v=\frac{1}{\sqrt{L_{0} C_{0}}}=\frac{1}{\sqrt{\mu_{0} \varepsilon_{0}}}=c,
$$

where $c$-velocity of propagation of light in the vacuum.

The wave drag of the line examined will be equal 


$$
Z=\frac{a}{b} \sqrt{\frac{\mu_{0}}{\varepsilon_{0}}}=\frac{a}{b} Z_{0},
$$

where $Z_{0}=\sqrt{\frac{\mu_{0}}{\varepsilon_{0}}}$ - wave drag of free space.

With the observance of the condition $a=b$ we obtain the equality $L_{0}=\mu_{0}$. This means that magnetic permeability plays the role of the longitudinal specific inductance of vacuum. In this case is observed also the equality $C_{0}=\varepsilon_{0}$. This means that the electric constant $\varepsilon_{0}$ plays the role of the transverse specific capacity of vacuum. In this interpretation both $\mu_{0}$ and $\varepsilon_{0}$ acquire clear physical sense and, just as in the long line, ensure the process of the propagation of electromagnetic wave in the free space.

The examination of electromagnetic wave in the long line can be considered as filling of space, which is been located between its conductors, special form of material, which present the electrical and magnetic fields. Mathematically it is possible to consider that these fields themselves possess specific energy and with their aid it is possible to transfer energy by the transmission lines. But if we examine the processes, which take place with the emission of electromagnetic waves with the aid of any antenna, then it possible to examine also as the filling of free space with this form of material. However, fields on geometric form and currents in this case it will be more complexly, since they will always be present both transverse and longitudinal component fields on. This approach excludes the need for application, for describing the propagation of electromagnetic waves, this substance as ether.

If we to the examined line of infinite length, or of line of that loaded with wave drag, connect the dc power supply $U$, then the field strength in the line will comprise:

$$
E_{y}=\frac{U}{a},
$$

and the current, which flows into the line from the power source, will be determined by the relationship:

$$
I=\frac{U}{Z}=\frac{a E_{y}}{Z} .
$$

Magnetic field in the line will be equal to the specific current, flowing in the line

$$
H_{x}=\frac{I}{b}=\frac{a E_{y}}{b Z} .
$$

substituting here the value $Z$, we obtain

$$
H_{x}=\frac{E_{y}}{Z_{0}} .
$$

The same connection between the electrical and magnetic field exists also for the case of the transverse electromagnetic waves, which are extended in the free space.

Comparing expressions for the energies, it is easy to see that the specific energy can be expressed through the electrical and magnetic fields

$$
\frac{1}{2} \mu_{0} H_{x}^{2}=\frac{1}{2} \varepsilon_{0} E_{y}^{2} \text {. }
$$


This means that the specific energy, accumulated in the magnetic and electric field in this line is identical. If the values of these energies are multiplied by the volumes, occupied by fields, then the obtained values coincide with expressions (13.3), (13.4).

Thus, it is possible to make the conclusion that in the line examined are propagated the same transverse plane waves, as in the free space. Moreover this conclusion is obtained not by the method of solution of Maxwell equations, but by the way of examining the dynamic processes, which are related to the discharge of parametric self-induction. The special feature of this line will be the fact that in it, in contrast to the free space, the stationary magnetic and electric fields can be extended, but this case cannot be examined by the method of solution of Maxwell equations.

Consequently, conditionally it is possible to consider that the long line is the device, which with the connection to it of dc power supply is filled up with two forms of the energy: electrical and magnetic. The specific densities of these energies are equal, and since and electrical and magnetic energy fill identical volumes, the general energy, accumulated in these fields is identical. The special feature of this line is the fact that with the flow in the line of direct current the distribution of electrical and magnetic fields on in it is uniform. It is not difficult to show that the force, which acts on the conductors of this line, is equal to zero. This follows from relationship (13.11), in which its right and leftist of part present the force gradients, applied to the planes of line. But electrical and magnetic forces have different signs; therefore they compensate each other. This conclusion concerns the transmission lines of any other configuration.

If we to the line exert the voltage, which is changed in the course of time according to any law $U(t)=a E_{y}(t)$, the like of analogy (13.5) it is possible to write down

$$
E_{y}(z)=E_{y}\left(t-\frac{z}{c}\right) \text {. }
$$

Analogous relationship will be also fields on for the magnetic.

Is obvious that the work $I(t) U(t)$ represents the power $P$, transferred through the cross section of line in the direction $z$. If in this relationship current and voltage was replaced through the tensions of magnetic and electrical fields on, then we will obtain $P=a b E_{y} H_{x}$. The work $E_{y} H_{x}$ represents the absolute value of Pointing vector, which represents the specific power, transferred through the cross section of the line of single area. Certainly, all these relationships can be written down also in the vector form.

Thus, all conclusions, obtained on the basis of the examination of processes in the long line by two methods, coincide. Therefore subsequently, without risking to commit the errors of fundamental nature, it is possible for describing the processes in the long lines successfully to use such parameters as the distributed inductance and capacity. Certainly, in this case one should understand that $C_{0}$ and this $L_{0}$ some integral characteristics, which do not consider structure fields on. It should be noted that from a practical point of view, the application of the parameters $C_{0}$ and $L_{0}$ has important significance, since. can be approximately solved the tasks, 
which with the aid of Maxwell equations cannot be solved. This, for example, the case, when spirals are the conductors of transmission line.

The importance of the obtained results consists in the fact that it is possible, without resorting to Maxwell equations, to solve the problems of propagation, is also shown that in the long lines and in the free space the electromagnetic processes are extended with the final speed.

The regularities indicated apply to all forms of transmission lines. For different types of lines the linear parameters depend on their sizes. For an example let us examine the coaxial line, whose linear capacity and inductance are expressed by the relationships:

$$
C_{0}=\frac{2 \pi \varepsilon_{0}}{\ln \left(\frac{D}{d}\right)}, \quad L_{0}=\frac{\mu_{0}}{2 \pi} \ln \left(\frac{D}{d}\right),
$$

where $D, d$ the inside diameter of the cylindrical part of the coaxial and the outer diameter of central core respectively. The introduced linear parameters, can be named field, since the discussion deals with that energy, which is stored up in the electrical and magnetic fields. However, the circumstance is not considered with this approach that besides field inductance there is still a kinetic inductance, which is obliged to kinetic energy of the moving charges. In the real transmission lines kinetic inductance is not calculated on the basis of that reason, that their speed is small in view of the very high density of current carriers in the conductors and therefore field inductance always is considerably greater than kinetic.

With the current $I$, which flows along the center conductor of coaxial line, energy accumulated in the specific inductance and linear inductance are connected with the relationship

$$
W_{L}=\frac{1}{2} L_{0} I^{2}=\frac{\mu_{0}}{4 \pi} \ln \left(\frac{D}{d}\right) I^{2} .
$$

We will consider that the current is evenly distributed over the section of center conductor. Then kinetic energy of charges in the conductor of unit length composes

$$
W_{k}=\frac{\pi d^{2} n m v^{2}}{8},
$$

where $n, m, v$ - electron density, their mass and speed respectively.

If one considers that $I=\frac{n e v \pi d^{2}}{4}$, then it is possible to write down

$$
W_{L}=\frac{1}{2} L_{0} I^{2}=\frac{\mu_{0}}{4 \pi} \ln \left(\frac{D}{d}\right) \frac{n^{2} e^{2} v^{2} \pi^{2} d^{4}}{16} .
$$

From these relationships we obtain, that to the case, when

$$
W_{k} \geq W_{L},
$$

the condition corresponds

$$
L_{k}=\frac{m}{n e^{2}} \geq \frac{\mu_{0}}{8} \ln \left(\frac{D}{d}\right) d^{2}
$$


where $L_{k}=\frac{m}{n e^{2}}$ - specific kinetic inductance of charges.

Hence we find that the fulfillment of conditions is necessary for the kinetic beams

$$
n \leq \frac{8 m}{d^{2} e^{2} \mu_{0}}
$$

in such a way that the flow would be kinetic, is necessary that the specific kinetic inductance would exceed linear inductance, which is carried out with the observance of the given condition. From this relationship it is possible to estimate, what electron density in the flow corresponds to this of the case.

Let us examine the concrete example: $d=1$ м, $\ln \left(\frac{D}{d}\right)=2$, then for the electron density in the beam must be satisfied the condition

$$
n \leq \frac{8 m}{e^{2} \mu_{0} \ln \left(\frac{D}{d}\right) d^{2}} \approx 10^{20} \frac{1}{M^{3}} .
$$

Such densities are characteristic to electron beams, and they are considerably lower than electron density in the conductors. Therefore electron beams should be carried to the kinetic flows, while electronic current in the conductors they relate to the potential flows. Therefore for calculating the energy, transferred by electromagnetic fields they use Pointing vector, and for calculating the energy, transferred by electron beams is used kinetic energy of separate charges. this all the more correctly, when the discussion deals with the calculation of the energy, transferred by ion beams, since. the mass of ions many times exceeds the mass of electrons.

Thus, the reckoning of the flows of charges to one or the other form depends not only on density and diameter of beam itself, but also on the diameter of that conducting tube, in which it is extended. It is obvious that in the case of potential beam, its front cannot be extended at a velocity, which exceeds the speed of light. It would seem that there are no such limitations for the purely kinetic beams. There is no clear answer to this question as yet. The mass of electron to usually connect with its electric fields and if we with the aid of the external conducting tube begin to limit these fields, then the mass of electron will begin to decrease, but the decrease of mass will lead to the decrease of kinetic inductance and beam will begin to lose its kinetic properties. And only when the part of the mass of electron does not have electrical origin, there is the hope to organize the purely kinetic electron beam, whose speed can exceed the speed of light. If we take the beam of protons, then picture will be the same. But here, if we take, for example, the nuclei of deuterium, which contain the neutron, whose mass is located, but electrical fields on then with the aid of such nuclei it is possible to organize purely kinetic beams, and it is possible to design for the fact that such beams can be driven away to the speeds of the large of the speed of light. If we let out this beam from the limiting tube into the free space, i.e. to attempt to convert it from the kinetic into the potential, then Cerenkov radiation of the type of that can be obtained, when electronic flux falls on Medium, where the phase speed of electromagnetic wave is lower than the speed of electron beam. 


\section{$\S 14$. Transient processes in the sections of the long lines}

Faraday established the law of induction, carrying out experiments on the solenoids, including turning off in them current, or moving with respect to the solenoids the turns of the wire, to which was connected the galvanometer. Its point of view, which is considered accurate and today, was reduced to the fact that with the connection to the solenoid of the dc power supply $U$, then current in all its turns increases according to the linear law

$$
I=\frac{U t}{L}
$$

where $L$ - inductance of solenoid.

Consequently, magnetic field with this interpretation for entire elongation of solenoid will increase synchronous. However, so whether this in reality. In order to be dismantled at this, let us examine a question about how swelling current in the shortened out section of long line will.

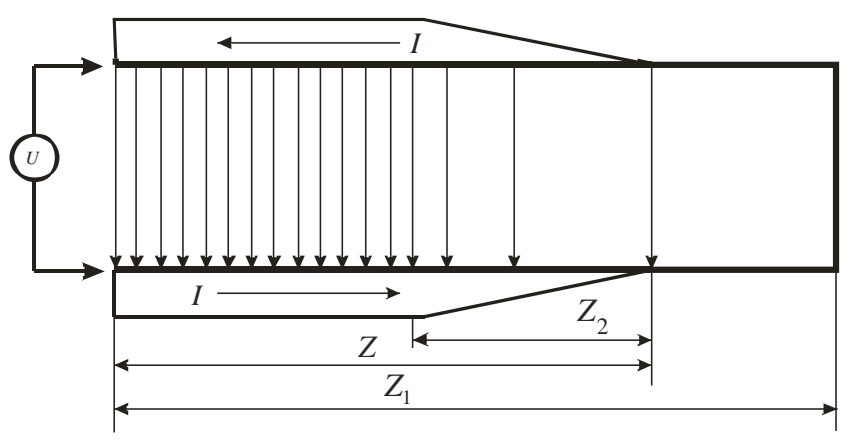

Fig. 8. Propagation of the current wave and voltage in the long line

If we the line, depicted to Fig. 2 , short out at a distance $z_{1}$, i.e. summary inductance will compose the value $L_{\Sigma}=z_{1} L_{0}=z_{1} \frac{a}{b} \mu_{0}$. If we connect to the line dc power supply, in it will begin to be extended the wave of the voltage $U$ and current $I=\frac{U}{Z}$ as shown in Fig. 8. The wave of voltage in its right part has the transition section $z_{2}$, which is named the front of the wave of voltage. This section corresponds to the transit time $\tau=\frac{z_{2}}{c}$, for which the voltage of the source, connected to the line, attains its nominal value.

Simultaneously with the wave of voltage in the line will move current wave. Specifically, in this transition section proceeds the acceleration of the charges from the zero speed in its beginning, to the values necessary for creating the rated current in the line, whose value is determined by the relationship $I=\frac{U}{Z}$. To this section is applied the voltage of the power source. In this case it is accepted that during the voltage transient increases according to the linear law (although in the general case it can increase according to any other law). It is accepted also that the 
time of this transient process is considerably less than the time, for which the front of voltage passes along the line to one side. Interval $z_{2}$ corresponds to the transient process, which is connected with the inertia properties of the device, which connects the voltage source to the line, or is created artificially by the way of increasing the voltage according to the specific law. It is assumed that $z_{1}$ is considerably greater than $z_{2}$. At the moment of the time, when on the cross connection in the end of the line appears the front of the voltage $U$, appears the wave with the voltage of reflected $-U$, which runs in the opposite direction. Since current in the wave reflected is equal to voltage with the negative sign and it moves in the opposite direction, then the summed current, created by this wave will be equal $-\left(-\frac{U}{Z}\right)=\frac{U}{Z}$, i.e., it there will be leak in the same direction as the current of the incident wave. Thus, the wave reflected, moving in the opposite direction, will leave after itself current, equal $\frac{2 U}{Z}$, and zero voltage. When the front of voltage to return at the beginning to line, it brings with itself the state of the doubled initial current and zero voltage. Source will again send into the front line of the voltage $U$ and current $\frac{U}{Z}$. This current will be formed with the current $\frac{2 U}{Z}$, and summed current in the line will compose $\frac{3 U}{Z}$. Current will further increase by steps, adding each sequential time to its previous value the value $\frac{2 U}{Z}$. If this process reflected in the time, thus will appear, as shown in Fig. 7. In this figure the time

$$
T=\frac{z_{1}}{c}=z_{1} \sqrt{L_{0} C_{0}}=z_{1} \sqrt{\mu_{0} \varepsilon_{0}} .
$$

It is equal to the time, for which the front of voltage passes along the line to one side beginning to the shortened out section.

The special feature of this process is that that the selection of energy from the voltage source will not be subordinated to linear law, but it will have spasmodic nature. The power, selected in the range of time from zero to $2 T$, will be equal $\frac{U^{2}}{Z}$ But in each subsequent interval of the time, equal $2 T$, it will grow already to the value $\frac{2 U^{2}}{Z}$. Thus, the growth of current bears completely not linear, but spasmodic nature. The process indicated occurs with any length of line. With the small length of gallop they follow through the small time intervals and the dependence of current on the time approximately it is possible to consider it linear that also is characteristic for the elements with the lumped parameters.

Should be focused attention on the fact that, the power, selected by the shortened out line in the source of voltage (Fig. 9), it is not linear function, but after the time 
equal $2 T$ abruptly it increases by $\frac{2 U^{2}}{Z}$, the first of gallops corresponding to the selected power $\frac{U^{2}}{Z}$. up to the moment $T$ it will reach the value $\Phi_{B}=\frac{z_{1}}{c} U$.

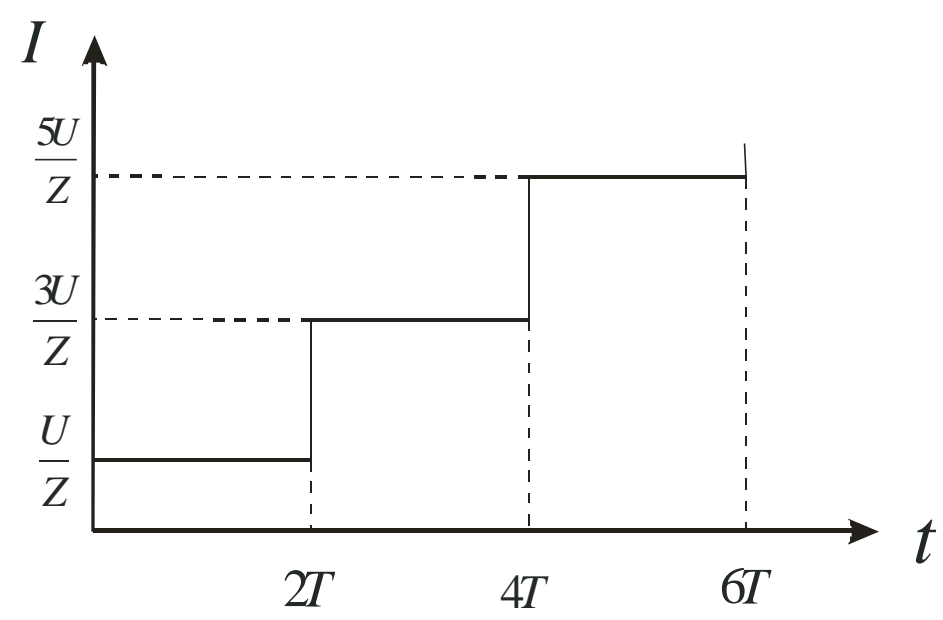

Fig. 9. Dependences of input current on the time in shortened line

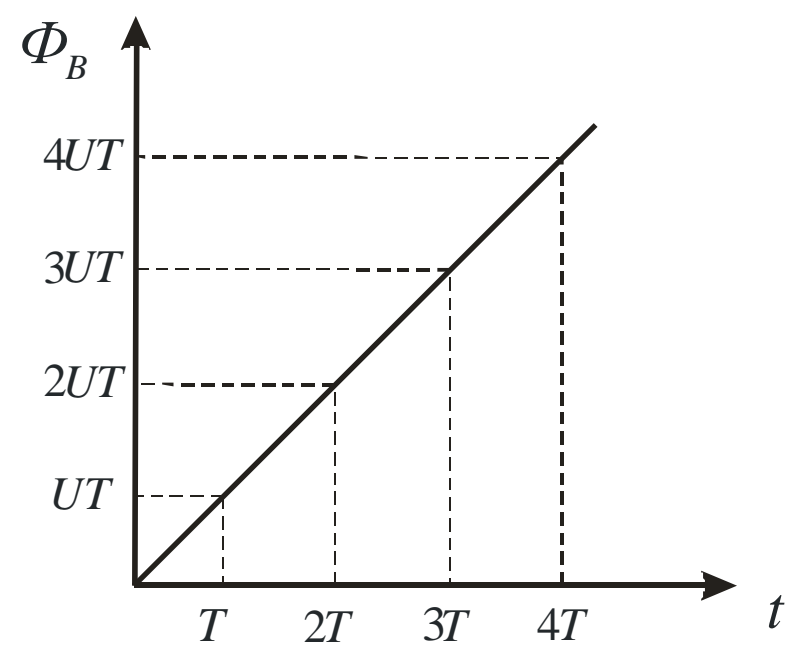

Fig. 10. Dependence of magnetic flux on the time in the shortened out line.

Is not difficult to show that the magnetic flux in this case changes according to the linear law (Fig. 10). Actually, during the forward stroke, to the moment of reaching by the wave of the shortened out section, flow will increase in the linear law, and

When, after being reflected from the shortened out section, the front of voltage will begin to move in the opposite direction, then flow will continue to grow according to the linear law, and up to the moment of the arrival of the front of voltage at the voltage source it will conversely reach the value $\Phi_{B}=\frac{2 z_{1}}{c} U$ Thus, with the connection of the shortened out line to the voltage source is carried out the law of induction $U=\frac{d \Phi_{B}}{d t}$. 
The electric flux in the line will also change, but according to another law (Fig. 11).

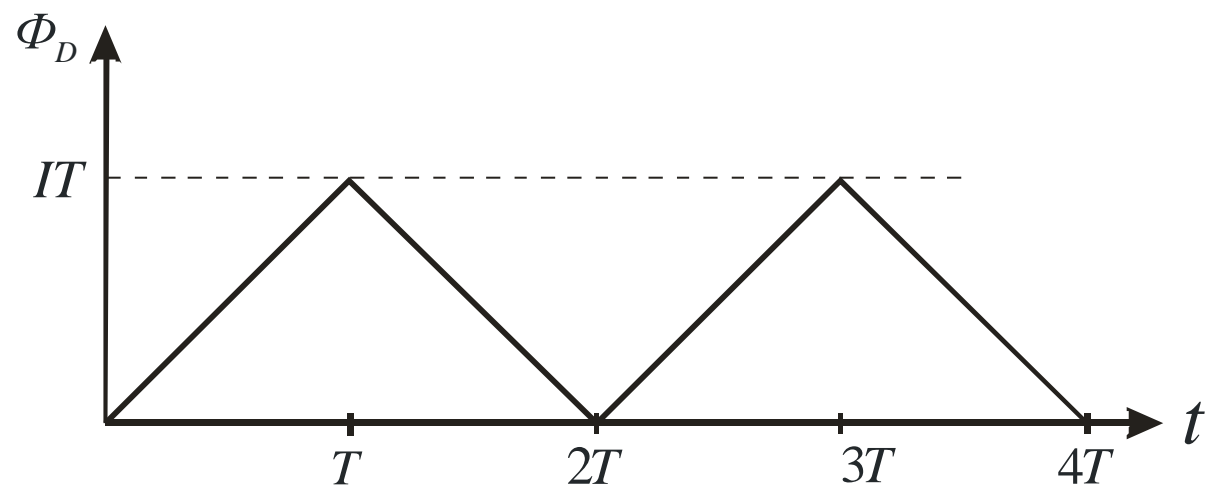

Fig. 11. Dependence of the electric flux on the time for the shortened out line

In contrast to the magnetic flux it will change periodically, first, growing, then, diminishing, according to the linear law. When wave moves in the positive direction, simultaneously grows magnetic and electric flux. In this case, both in the magnetic and in the electric field stored energy grows. When wave begins to move in the opposite direction, then electric field begins to disappear, and its energy passes into the magnetic energy of reverse current wave. After the front of the wave of voltage reaches incoming line, magnetic field and current in it doubles, and electric field disappears. Further cycle is repeated. Consequently, the process of the growth of magnetic flux in that shortened outed by long line, in the required order accompanies the process of an alternation in the flow of electrical induction, as a result of which between the planes of line periodically it appears and it disappears electric field.

Let us assume that line is made from superconductor and loss-free. Then after replacing at the specific moment the voltage generator with the superconductive cross connection, it is possible to freeze current in the line. The moment, when in the line electric field completely is absent is favorable moment for this procedure. Then in the line is frozen the flow $\Phi_{B}=\frac{2 N z_{1}}{c} U$, to which will correspond the current $I=\frac{2 N U}{Z}$. What will be, if the voltage source by the superconductive cross connection was replaced at that moment of the time, when in the line is found the front of voltage and what- t. e section it is filled with electric fields? In this case this section will move in the line, being alternately reflected thus one, thus another end of the shortened out line, until it spends its energy for the emission. Only integral (quantized) value of flow and current in accordance with the given relationships can be frozen for this reason in the line shortened out from both sides.

This phenomenon is an example of the macroscopic quantization of flow in the macroscopic structures, which have the specific sizes. The same quantization of flow occurs also in the microscopic structures, which the atoms are.

From the point of view of chains with the lumped parameters, growth of current in the solenoid with the connection to it of the voltage source occur according to the linear law, moreover in all its turns simultaneously. But so whether this? For ex- 
plaining this question let us replace the upper plane of the two-wire circuit (Fig. 12) by long solenoid. If we to this line connect the voltage source, then the process of the growth of current in it will in no way differ from that examined. The linear inductance of line will be now in essence determined by the linear inductance of solenoid and the velocity of propagation and current wave, and the wave of the voltage (voltage now it will be applied between the solenoid and the lower conductor of line) it will be less than in the preceding case.

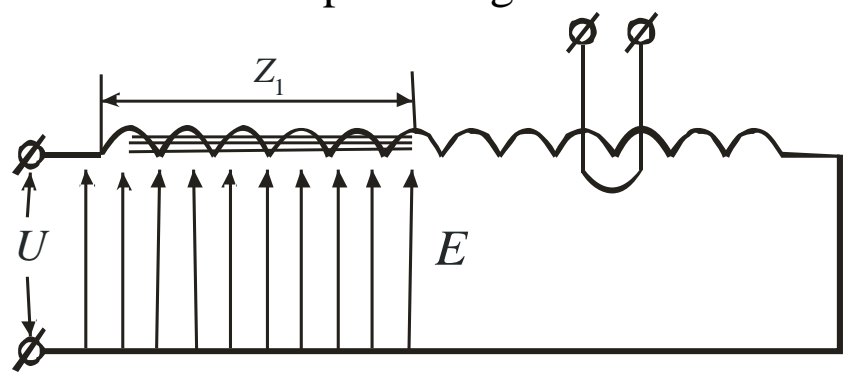

Fig. 12. The diagram of the propagation of fields in the long solenoid.

When in the line examined wave will reach the point with the coordinate $z_{1}$, then magnetic field fill only the part of the solenoid, located between the power source and the point $z_{1}$. When wave reaches its end, then magnetic field fill entire solenoid. The magnetic field in the solenoid will be doubled with the back stroke of wave, and process will begin first.

Let us place now that the solenoid in the specific place is enveloped by turn. This process is such to mechanical dressing of the covering turn to the end of the solenoid with the only difference that in this case magnetic flux, being moved inside the solenoid, itself it pierces its covering turn. Moreover the speed of the motion of the front of magnetic flux in this case is incommensurably more than during mechanical dressing. But both processes have identical nature. By these processes is explained the phenomenon of the mutual induction between the solenoid and the covering turn. The pulse separation is small with the small length of solenoid; therefore they, merging, is formed almost constant voltage. Voltage in the turn will be induced only at the moment of the intersection with the magnetic flux of the solenoid of the environments of the cross section, enveloped by turn. In the environments of the covering turn will at this moment appear both the vector potential and the magnetic field. And, precisely, the intersection of the covering turn with the magnetic fields of the scattering (the same, as at the end of the solenoid with the direct current) leads to the induction in it emf This moment will begin both with the straight line and with the back stroke of wave; moreover the polarity of the voltage pulses, induced in the turn, in both cases will be one and the same. The frequency of these pulses will depend on the length of solenoid, and it will be the greater, the shorter the solenoid. Consequently, the average value of the induced voltage will be grow with the decrease of the length of solenoid, i.e., its quantity of turns, that also determines the transformation ratio of this transformer.

After examining the process of the growth of currents fields on in the long solenoid, let us return to the problem of the presence of the circulation of vector poten- 
tial around the long solenoid. Let us give the existing point of view on this question, represented in the work [4]. The value of vector potential in the space, which surrounds solenoid, is found from the relationship

$$
A(r)=\frac{n I r_{0}{ }^{2}}{2 \varepsilon_{0} c^{2} r},
$$

where $n$ - quantity of turns, which falls per unit of the length of solenoid, $I$ - the current, which flows through the solenoid, $r_{0}$ - diameter of solenoid, $r$ - distance from the axis of solenoid to the observation point. It is assumed during the record of this relationship that $r \geq r_{0}$.

The inductance of solenoid is determined by the expression

$$
L=\frac{n^{2} \pi r_{0}^{2} z_{0}}{\varepsilon_{0} c^{2}},
$$

where $z_{0}$ - length of solenoid.

If we to the solenoid connect the dc power supply $U$, then taking into account relationships (14.1)-(14.3), we obtain

$$
A(r, t)=\frac{U t}{2 \pi N r},
$$

where $N$ - total number of turns in the solenoid, and since

$$
E=-\mu_{0} \frac{\partial A}{\partial t},
$$

that the tension of electric field in the environment of solenoid at the moment of the connection to it of dc power supply will comprise

$$
E(r)=\frac{\mu_{0} U}{2 \pi N r} .
$$

The tension of electric field in accordance with the version in question appears at the moment of connection to the solenoid of the power source instantly for entire its elongation. If the solenoid lacks resistance, then the tension of electric field will be constant during entire period of the time of connection to the solenoid of dc power supply. What here do appear contradictions? First, electric fields possess energy, and instantly they cannot appear. The second contradiction escapes from the first and consists in the fact that, since the electric fields possess energy, this energy must be included in the general energy, accumulated in the solenoid. But only magnetic fields inside the solenoid are considered with the calculation of this energy.

Thus, very process of inducting the electrical fields on around the long solenoid it occurs in no way in the manner that this represented in the existing literature [4], when it is considered that the circulation of magnetic vector potential for entire its elongation grows simultaneously, that also leads to the induction emf in the covering turn. 


\section{PART II \\ NEW IDEAS IN THE CLASSICAL ELECTRODYNAMICS}

\section{CHAPTER 4 \\ NEW APPROACHES AND DETERMINATIONS}

New ideas do not appear at the empty place, accumulation precedes their appearance and comprehension is previously the obtained results. The fact that classical electrodynamics consists in fact of several, the not connected together parts, it cannot but cause uneasiness. The fact that some electrodynamic effects cannot be explained within the framework to unified theory, also speaks, that classical electrodynamics as yet it is not possible to consider as the final physical theory. In the previous divisions it was shown, to what extent the role of magnetic vector potential in the electrodynamics was great, were examined the nonjoinings, which appear with the explanation of the appearance of electrical fields on around the long solenoid with an attempt at the use of this parameter. But this state of affairs is brought into question and very law of the Faraday induction, since and vector potential and magnetic field and magnetic flux in the solenoid the closely related values. In Faraday's times were still known neither equations of Maxwell nor that that the electromagnetic fields are extended with the final speed, and consequently these effects, which are also tightly connected with the processes of induction also they could not be taken into account. Even Maxwell, after writing down its famous equations it could not experimentally prove existence of electromagnetic waves. Hertz made this for the first time, after creating by very simple method the first in the world SHF generator [37], and with the aid of this procedure were obtained the salient results, after showing that the electromagnetic waves can be extended not only in the free space, but also can with the final speed be extended along the metallic conductors. As it was shown in the previous divisions, the use of the substantional derivative when writing the laws of induction provides a Lorentz force of these laws, and not to introduce it as a postulate. This way we will attempt to outline in this division, after building the bases of such noncontradictory electrodynamics, which will combine its odd parts and that united basis, which is its foundation, will be indicated.

\section{$\S 15$. Dynamic potentials and the field of the moving charges}

With the propagation of wave in the long line it is filled up with two forms of energy, which can be determined through the currents and the voltages or through the electrical and magnetic fields in the line. And only after wave will fill with electromagnetic energy all space between the generator and the load on it will begin to be separated energy. I.e. the time, by which stays this process, generator expended its power to the filling with energy of the section of line between the generator and the load. But if we begin to move away load from incoming line, then a quantity of energy being isolated on it will decrease, since. the part of the energy, expended 
by source, will leave to the filling with energy of the additional length of line, connected with the motion of load [38]. If load will approach a source, then it will obtain an additional quantity of energy due to the decrease of its length. But if effective resistance is the load of line, then an increase or the decrease of the power expendable in it can be connected only with a change in the voltage on this resistance. Therefore we come to the conclusion that during the motion of the observer of those of relatively already existing in the line fields on must lead to their change. The productivity of this approach with the application of Galileo conversions will be demonstrated in this chapter.

Being located in assigned IRS, us interest those fields, which are created in it by the fixed and moving charges, and also by the electromagnetic waves, which are generated by the fixed and moving sources of such waves. The fields, which are created in this IRS by moving charges and moving sources of electromagnetic waves, we will call dynamic. Can serve as an example of dynamic field the magnetic field, which appears around the moving charges.

As already mentioned, in the classical electrodynamics be absent the rule of the conversion of electrical and magnetic fields on upon transfer of one inertial system to another. This deficiency removes SR, basis of which are the covariant Lorenz conversions. With the entire mathematical validity of this approach the physical essence of such conversions up to now remains unexplained [39].

In this division will made attempt find the precisely physically substantiated ways of obtaining the conversions fields on upon transfer of one IRS to another, and to also explain what dynamic potentials and fields can generate the moving charges. The first step, demonstrated in the works [33, 40], was made in this direction a way of the introduction of the symmetrical laws of magnetoelectric and electromagnetic induction. These laws are written as follows [22, 40-43]:

$$
\begin{aligned}
& \int\left[\mathbf{E}^{\prime} d l^{\prime}=-\int \frac{\partial \mathbf{B}}{\partial t} d \mathbf{s}+\int\left[[\mathbf{v} \times \mathbf{B}] d l^{\prime}\right.\right. \\
& \int f \mathbf{H}^{\prime} d l^{\prime}=\int \frac{\partial \mathbf{D}}{\partial t} d \mathbf{s}-\int\left[\int[\mathbf{v} \times \mathbf{D}] d l^{\prime}\right.
\end{aligned}
$$

or

$$
\begin{aligned}
& \operatorname{rot} \mathbf{E}^{\prime}=-\frac{\partial \mathbf{B}}{\partial t}+\operatorname{rot}[\mathbf{v} \times \mathbf{B}] \\
& \operatorname{rot} \mathbf{H}^{\prime}=\frac{\partial \mathbf{D}}{d t}-\operatorname{rot}[\mathbf{v} \times \mathbf{D}]
\end{aligned}
$$

For the constants fields on these relationships they take the form:

$$
\begin{aligned}
& \mathbf{E}^{\prime}=[\mathbf{v} \times \mathbf{B}] \\
& \mathbf{H}^{\prime}=-[\mathbf{v} \times \mathbf{D}]
\end{aligned} .
$$

In relationships (15.1)-(15.3), which assume the validity of the Galileo conversions, prime and not prime values present fields and elements in moving and fixed IRS respectively. It must be noted, that conversions (16.3) earlier could be obtained only from the Lorenz conversions. 
The relationships (15.1)-(15.3), which present the laws of induction, do not give information about how arose fields in initial fixed IRS. They describe only laws governing the propagation and conversion fields on in the case of motion with respect to the already existing fields.

The relationship (15.3) attest to the fact that in the case of relative motion of frame of references, between the fields $\mathbf{E}$ and $\mathbf{H}$ there is a cross coupling, i.e., motion in the fields $\mathbf{H}$ leads to the appearance fields on $\mathbf{E}$ and vice versa. From these relationships escape the additional consequences, which were for the first time examined in the work [9]. If the charged rod has a linear charge $g$, its electric field $E=\frac{g}{2 \pi \varepsilon r}$ decreases according to the law $\frac{1}{r}$, where $r$ is distance from the central axis of the rod to the observation point.

If we in parallel to the axis of rod in the field $E$ begin to move with the speed $\Delta v$ another IRS, then in it will appear the additional magnetic field $\Delta H=\varepsilon E \Delta v$. If we now with respect to already moving IRS begin to move third frame of reference with the speed $\Delta v$, then already due to the motion in the field $\Delta H$ will appear additive to the electric field $\Delta E=\mu \varepsilon E(\Delta v)^{2}$. This process can be continued and further, as a result of which can be obtained the number, which gives the value of the electric field $E_{v}^{\prime}(r)$ in moving IRS with reaching of the speed $v=n \Delta v$, when $\Delta v \rightarrow 0$, and $n \rightarrow \infty$. In the final analysis in moving IRS the value of dynamic electric field will prove to be more than in the initial and to be determined by the relationship:

$$
E^{\prime}\left(r, v_{\perp}\right)=\frac{g \operatorname{ch} \frac{v_{\perp}}{c}}{2 \pi \varepsilon r}=E \operatorname{ch} \frac{v_{\perp}}{c} .
$$

If speech goes about the electric field of the single charge $e$, then its electric field will be determined by the relationship:

$$
E^{\prime}\left(r, v_{\perp}\right)=\frac{e c h \frac{v_{\perp}}{c}}{4 \pi \varepsilon r^{2}}
$$

where $v_{\perp}$ - normal component of charge rate to the vector, which connects the moving charge and observation point.

Expression for the scalar potential, created by the moving charge, for this case will be written down as follows:

$$
\varphi^{\prime}\left(r, v_{\perp}\right)=\frac{e c h \frac{v_{\perp}}{c}}{4 \pi \varepsilon r}=\varphi(r) \operatorname{ch} \frac{v_{\perp}}{c},
$$

where $\varphi(r)$ - scalar potential of fixed charge. The potential $\varphi^{\prime}\left(r, v_{\perp}\right)$ can be named scalar-vector, since it depends not only on the absolute value of charge, but also on speed and direction of its motion with respect to the observation point. Maximum value this potential has in the direction normal to the motion of charge itself. Moreover, if charge rate changes, which is connected with its acceleration, then can be calculated the electric fields, induced by the accelerated charge.

During the motion in the magnetic field, using the already examined method, we obtain: 


$$
H^{\prime}\left(v_{\perp}\right)=H \operatorname{ch} \frac{v_{\perp}}{c} .
$$

where $v_{\perp}$ - speed normal to the direction of the magnetic field. The same result can be obtained by another method.

Let us designate field variables in the fixed frame of reference without the prime, and in the mobile - with the prime. In the differential form let us write down the formulas of the mutual induction of electrical and magnetic fields on in the mobile frame of reference as follows:

$$
\begin{aligned}
d H^{\prime} & =\varepsilon E^{\prime} d v_{\perp}, \\
d E^{\prime} & =\mu H^{\prime} d v_{\perp} .
\end{aligned}
$$

Or otherwise,

$$
\begin{aligned}
& \frac{d H^{\prime}}{d v_{\perp}}=\varepsilon E^{\prime}, \\
& \frac{d E^{\prime}}{d v_{\perp}}=\mu H^{\prime},
\end{aligned}
$$

where (15.7) it corresponds (15.5), and (15.8) it corresponds (15.6).

After dividing equations (15.7) and (15.8) on $E$ and $H$, we will obtain respectively:

$$
\begin{aligned}
& \frac{d\left(H^{\prime} / E\right)}{d v_{\perp}}=\varepsilon \frac{E^{\prime}}{E}, \\
& \frac{d\left(E^{\prime} / E\right)}{d v_{\perp}}=\mu \frac{H^{\prime}}{H} .
\end{aligned}
$$

Differentiating both parts (15.10), we have:

$$
\frac{d^{2}\left(E^{\prime} / E\right)}{d^{2} v_{\perp}}=\mu \frac{d\left(H^{\prime} / E\right)}{d v_{\perp}} .
$$

After substituting (15.9) in (15.11), we will obtain:

$$
\frac{d^{2}\left(E^{\prime} / E\right)}{d^{2} v_{\perp}}=\mu \varepsilon \frac{E^{\prime}}{E} .
$$

The function is the general solution (15.12) of differential equation

$$
\frac{E^{\prime}}{E}=C_{2} c h\left(\frac{v_{\perp}}{c}\right)+C_{1} s h\left(\frac{v_{\perp}}{c}\right),
$$

where $c$ - the speed of light, $C_{1}, C_{2}-$ arbitrary constants.

Since with $v_{\perp}=0$ must be made $E^{\prime}=E$, that from (15.13) we will obtain:

$$
C_{2}=1 \text {. }
$$

After substituting (15.14) in (15.13), we finally have the general solution, into which enters one arbitrary constant $C_{1}$ :

$$
\frac{E^{\prime}}{E}=\operatorname{ch}\left(\frac{v_{\perp}}{c}\right)+C_{1} s h\left(\frac{v_{\perp}}{c}\right) .
$$

Selecting $C_{1}=0$, we obtain

$$
E^{\prime}=\operatorname{Ech}\left(\frac{v_{\perp}}{c}\right) .
$$


If we apply the obtained results to the electromagnetic wave and to designate components fields on parallel speeds IRS as $E_{\uparrow}, H_{\uparrow}$, and $E_{\perp}, H_{\perp}$ as components normal to it, then conversions fields on they will be written down [9]:

$$
\begin{aligned}
& \mathbf{E}_{\uparrow}^{\prime}=\mathbf{E}_{\uparrow}, \\
& \mathbf{E}_{\perp}^{\prime}=\mathbf{E}_{\perp} \operatorname{ch} \frac{v}{c}+\frac{Z_{0}}{v}\left[\mathbf{v} \times \mathbf{H}_{\perp}\right] \operatorname{sh} \frac{v}{c}, \\
& \mathbf{H}_{\uparrow}^{\prime}=\mathbf{H}_{\uparrow}, \\
& \mathbf{H}_{\perp}^{\prime}=\mathbf{H}_{\perp} \operatorname{ch} \frac{v}{c}-\frac{1}{v Z_{0}}\left[\mathbf{v} \times \mathbf{E}_{\perp}\right] \operatorname{sh} \frac{v}{c},
\end{aligned}
$$

where $Z_{0}=\sqrt{\frac{\mu_{0}}{\varepsilon_{0}}}$ - impedance of free space, $c=\sqrt{\frac{1}{\mu_{0} \varepsilon_{0}}}$ - speed of light. Let us name conversions (15.15) the Mende conversions.

Let us give a stricter conclusion in the matrix form even let us show that the form of conversions is wholly determined by the type of the utilized law of addition of velocities - classical or relativistic. This method is proposed by a participant in the Scientific forum of movement for the revivals of domestic science to N. A. Drobyshev $[44,45]$.

Let us examine the totality IRS of such, that IRS $\mathrm{K}_{1}$ moves with the speed $\Delta v$ relative to IRS K, IRS $K_{2}$ moves with the same speed $\Delta v$ relative to $K_{1}$, etc. If the module of the speed $\Delta v$ is small (in comparison with the speed of light $\mathrm{s}$ ), then for the transverse components fields on in ISO $K_{1} K_{2}, \ldots$ we have:

$$
\begin{array}{ll}
\mathbf{E}_{1 \perp}=\mathbf{E}_{\perp}+\Delta \mathbf{v} \times \mathbf{B}_{\perp} & \mathbf{B}_{1 \perp}=\mathbf{B}_{\perp}-\Delta \mathbf{v} \times \mathbf{E}_{\perp} / c^{2} \\
\mathbf{E}_{2 \perp}=\mathbf{E}_{1 \perp}+\Delta \mathbf{v} \times \mathbf{B}_{1 \perp} & \mathbf{B}_{2 \perp}=\mathbf{B}_{1 \perp}-\Delta \mathbf{v} \times \mathbf{E}_{1 \perp} / c^{2}
\end{array}
$$

Upon transfer to each following IRS of field are obtained increases in $\Delta \mathbf{E}$ and $\Delta \mathbf{B}$

$$
\Delta \mathbf{E}=\Delta \mathbf{v} \times \mathbf{B}_{\perp}, \quad \Delta \mathbf{B}=-\Delta \mathbf{v} \times \mathbf{E}_{\perp} / c^{2},
$$

where the fields $\mathbf{E}_{\perp}$ and $\mathbf{B}_{\perp}$ relate to current ISO. Directing the Cartesian axis $x$ along $\Delta \mathbf{v}$, let us rewrite (15.17) in the components of the vector

$$
\Delta E_{y}=-B_{z} \Delta v, \quad \Delta E=B_{y} \Delta v, \quad \Delta B_{y}=E_{z} \Delta v / c^{2} .
$$

Relationship (15.18) can be represented in the matrix form

$$
\Delta U=A U \Delta v \quad\left(\begin{array}{lccc}
0 & 0 & 0 & -1 \\
0 & 0 & 1 & 0 \\
0 & 1 / c^{2} & 0 & 1 \\
-1 / c^{2} & 0 & 0 & 0
\end{array}\right) \quad U=\left(\begin{array}{l}
E_{y} \\
E_{z} \\
B_{y} \\
B_{z}
\end{array}\right) .
$$

If one assumes that the speed of system is summarized for the classical law of addition of velocities, i.e., the speed of final IRS $K^{\prime}=K_{N}$ relative to the initial $K$ is $v=N \Delta v$, then we will obtain the matrix system of the differential equations

$$
\frac{d U(v)}{d v}=A U(v)
$$


with the matrix of the system $v$ independent of the speed $A$. The solution of system is expressed as the matrix exponential curve $\exp (v A)$ :

$$
U^{\prime} \equiv U(v)=\exp (v A) U, \quad U=U(0),
$$

here $U$ - matrix column fields on in the system $K$, and $U^{\prime}$ - matrix column fields on in the system $K^{\prime}$. Substituting (15.20) in the system (15.19), we are convinced, what $U^{\prime}$ is actually the solution of the system (15.19):

$$
\frac{d U(v)}{d v}=\frac{d[\exp (v A)]}{d v} U=A \exp (v A) U=A U(v) .
$$

It remains to find this exponential curve by its expansion in the series:

$$
\exp (v a)=E+v A+\frac{1}{2 !} v^{2} A^{2}+\frac{1}{3 !} v^{3} A^{3}+\frac{1}{4 !} v^{4} A^{4}+\ldots
$$

where $E$ - unit matrix with the size $4 \times 4$. For this it is convenient to write down the matrix $A$ in the unit type form

$$
A=\left(\begin{array}{lr}
0 & -\alpha \\
\alpha / c^{2} & 0
\end{array}\right), \quad \alpha=\left(\begin{array}{ll}
0 & 1 \\
-1 & 0
\end{array}\right), \quad 0=\left(\begin{array}{ll}
0 & 0 \\
0 & 0
\end{array}\right) .
$$

Then

$$
\begin{array}{rlrl}
A^{2} & =\left(\begin{array}{cc}
-\alpha^{2} / c^{2} & 0 \\
0 & -\alpha / c^{2}
\end{array}\right), & A^{3}=\left(\begin{array}{ccc}
0 & \alpha^{3} / c^{2} \\
-\alpha^{3} / c^{4} & 0
\end{array}\right), \\
A^{4}=\left(\begin{array}{cc}
\alpha^{4} / c^{4} & 0 \\
0 & \alpha^{4} / c^{4}
\end{array}\right), & A^{5}=\left(\begin{array}{ccc}
0 & -\alpha^{5} / c^{4} \\
\alpha^{5} / c^{6} & 0
\end{array}\right) \cdots .
\end{array}
$$

And the elements of matrix exponential curve take the form

$$
\begin{gathered}
{[\exp (v A)]_{11}=[\exp (v A)]_{22}=I-\frac{v^{2}}{2 ! c^{2}}+\frac{v^{4}}{4 ! c^{4}}-\ldots .} \\
{[\exp (v A)]_{21}=-c^{2}[\exp (v A)]_{12}=\frac{\alpha}{c}\left(\frac{v}{c} I-\frac{v^{3}}{3 ! c^{3}}+\frac{v^{5}}{5 ! c^{5}}-\ldots .\right),}
\end{gathered}
$$

where $I$ - the unit matrix $2 \times 2$. It is not difficult to see that $-\alpha^{2}=\alpha^{4}=-\alpha^{6}=\alpha^{8}=\ldots . .=I$; therefore we finally obtain

$$
\begin{gathered}
\exp (v A)=\left(\begin{array}{ccc}
I c h v / c & -c \alpha \operatorname{sh} v / c \\
(\alpha \operatorname{sh} v / c) / c & I \operatorname{ch} v / c
\end{array}\right)= \\
\left(\begin{array}{cccc}
\operatorname{ch} v / c & 0 & 0 & -c \operatorname{sh} v / c \\
0 & \operatorname{ch} v / c & \operatorname{csh} v / c & 0 \\
0 & (\operatorname{ch} v / c) / c & c h v / c & 0 \\
-(\operatorname{sh} v / c) / c 0 & 0 & \operatorname{ch} v / c
\end{array}\right) .
\end{gathered}
$$


Now we return to (15.20) and substituting there $\exp (v A)$, we find

$$
\begin{array}{ll}
E_{y}^{\prime}=E_{y} c h v / c-c B_{z} \operatorname{sh} v / c, & E_{z}^{\prime}=E_{z} \operatorname{ch} v / c+c B_{y} \operatorname{sh} v / c, \\
B_{y}^{\prime}=B_{y} c h v / c+\left(E_{z} / c\right) \operatorname{sh} v / c, & B_{z}^{\prime}=B_{z} \operatorname{ch} v / c-\left(E_{y} / c\right) s h v / c
\end{array} .
$$

Or in the vector record

$$
\begin{aligned}
& \mathbf{E}_{\perp}^{\prime}=\mathbf{E}_{\perp} \operatorname{ch} \frac{v}{c}+\frac{v}{c} \mathbf{v} \times \mathbf{B}_{\perp} \operatorname{sh} \frac{v}{c}, \\
& \mathbf{B}_{\perp}^{\prime}=\mathbf{B}_{\perp} \operatorname{ch} \frac{v}{c}-\frac{1}{v c} \mathbf{v} \times \mathbf{E}_{\perp} \operatorname{sh} \frac{v}{c}
\end{aligned}
$$

This is conversions (15.15)

Appears a regular question; therefore they are differed from the appropriate conversions fields on in the classical electrodynamics, indeed in it with the low speeds $\Delta \vec{v}$ occur initial relationships (15.16) and (15.17). The fact is that according to the relativistic law of addition of velocities, are added not speeds, but rapidities (https://ru.wikipedia.org/wiki/ Быстрота). According to definition the rapidity is introduced as

$$
\theta=\operatorname{arth} \frac{v}{c}
$$

Precisely, if the rapidity of the systems $K_{1}$ and $K, K_{2}$ and $K_{1}, K_{3}$ and $K_{2}$, etc., they are distinguished to $\Delta \theta$, then rapidity the rapidity IRS $K^{\prime}=K_{N}$ relative to $K$ is $\theta=N \Delta \theta$. With the low speeds $\Delta \theta \cong \Delta v$. Therefore formula (15.17) it is possible to write down so

$$
\Delta \mathbf{E}=\Delta \theta \times \mathbf{B}_{\perp}, \quad \Delta \mathbf{B}=-\Delta \boldsymbol{\theta} \times \mathbf{E}_{\perp} / c^{2},
$$

where $\boldsymbol{\theta}=\theta \frac{\mathbf{v}}{v}$. System (15.19) taking into account the additivity of rapidity, but not speed, it is substituted by the system of equations

$$
\frac{d U(\theta)}{d \theta}=A U(\theta)
$$

Thus, all computations will be analogous given above, only with the difference that in the expressions instead of the speeds will figure rapidity. In particular formulas (15.21) take the form

$$
\begin{aligned}
& \mathbf{E}_{\perp}^{\prime}=\mathbf{E}_{\perp} \operatorname{ch} \frac{\theta}{c}+\frac{\theta}{c} \boldsymbol{\theta} \times \mathbf{B}_{\perp} \operatorname{sh} \frac{\theta}{c}, \\
& \mathbf{B}_{\perp}^{\prime}=\mathbf{B}_{\perp} \operatorname{ch} \frac{\theta}{c}-\frac{1}{\theta c} \boldsymbol{\theta} \times \mathbf{E}_{\perp} \operatorname{sh} \frac{\theta}{c},
\end{aligned}
$$


or

$$
\begin{aligned}
& \mathbf{E}_{\perp}^{\prime}=\mathbf{E}_{\perp} \operatorname{ch} \frac{\theta}{c}+\frac{v}{c} \mathbf{v} \times \mathbf{B}_{\perp} \operatorname{sh} \frac{\theta}{c}, \\
& \mathbf{B}_{\perp}^{\prime}=\mathbf{B}_{\perp} \operatorname{ch} \frac{\theta}{c}-\frac{1}{v c} \mathbf{v} \times \mathbf{E}_{\perp} \operatorname{sh} \frac{\theta}{c},
\end{aligned}
$$

Since

$$
\operatorname{ch} \frac{\theta}{c}=\frac{1}{\sqrt{1-t^{2}(\theta / c)}}, \quad \operatorname{sh} \frac{\theta}{c}=\frac{t h(\theta / c)}{\sqrt{1-t^{2}(\theta / c)}},
$$

That substitution (15.22) in (15.23) leads to the well-known conversions fields on

$$
\begin{aligned}
& \mathbf{E}_{\perp}^{\prime}=\frac{1}{\sqrt{1-v^{2} / c^{2}}}\left(\mathbf{E}_{\perp}+\mathbf{v} \times \mathbf{B}_{\perp}\right) \\
& \mathbf{B}_{\perp}^{\prime}=\frac{1}{\sqrt{1-v^{2} / c^{2}}}\left(\mathbf{B}_{\perp}-\frac{1}{c^{2}} \mathbf{v} \times \mathbf{E}_{\perp}\right),
\end{aligned}
$$

with the small relative conversion rates (15.21) and (15.24) differ, beginning from the terms of the expansion of the order $v^{2} / c^{2}$.

We show how to use the relationships (15.15) it is possible to explain the phenomenon of phase aberration, which did not have within the framework existing classical electrodynamics of explanations. We will consider that there are components of the plane wave $H_{z}, E_{x}$, which is extended in the direction $y$, and primed system moves in the direction of the axis $x$ with the speed $v_{x}$. Then components fields on in the prime coordinate system in accordance with relationships (15.15) they will be written down:

$$
\begin{aligned}
& E_{x}^{\prime}=E_{x}, \\
& E_{y}^{\prime}=H_{z} \operatorname{sh} \frac{v_{x}}{c}, \\
& H_{z}^{\prime}=H_{z} \operatorname{ch} \frac{v_{x}}{c} .
\end{aligned}
$$

Thus, is a heterogeneous wave, which has in the direction of propagation the component $E_{v}^{\prime}$.

Let us write down the summary field $E^{\prime}$ in moving IRS:

$$
E^{\prime}=\left[\left(E_{x}^{\prime}\right)^{2}+\left(E_{y}^{\prime}\right)^{2}\right]^{\frac{1}{2}}=E_{x} \operatorname{ch} \frac{v_{x}}{c} .
$$

If the vector $\mathbf{H}^{\prime}$ is as before orthogonal the axis $y$, then the vector $\mathbf{E}^{\prime}$ is now inclined toward it to the angle $\alpha$, determined by the relationship: 


$$
\alpha \cong \operatorname{sh} \frac{v}{c} \cong \frac{v}{c}
$$

This is phase aberration. Specifically, to this angle to be necessary to incline telescope in the direction of the motion of the Earth around the sun in order to observe stars, which are located in the zenith.

The Pointing vector is now also directed no longer along the axis $y$, but being located in the plane $x y$, it is inclined toward the axis $y$ to the angle, determined by relationships (15.26). However, the relation of the absolute values of the vectors $\mathbf{E}^{\prime}, \mathbf{H}^{\prime}$ in both systems they remained identical. However, the absolute value of the very vector of Pointing increased. Thus, even transverse motion of inertial system with respect to the direction of propagation of wave increases its energy in the moving system. This phenomenon is understandable from a physical point of view. It is possible to give an example with the rain drops. When they fall vertically, then is energy in them one. But in the inertial system, which is moved normal to the vector of their of speed, to this speed the velocity vector of inertial system is added. In this case the absolute value of the speed of drops in the inertial system will be equal to square root of the sum of the squares of the speeds indicated. The same result gives to us relationship (15.25).

Is not difficult to show that, if we the polarization of electromagnetic wave change ourselves, then result will remain before. Conversions with respect to the vectors $\mathbf{E}, \mathbf{H}$ are completely symmetrical, only difference will be the fact that to now comes out the wave, which has to appear addition in the direction of propagation in the component $H_{v}^{\prime}$.

Such waves have in the direction of its propagation additional of the vector of electrical or magnetic field, and in this they are similar $E, H$ of the waves, which are extended in the waveguides. In this case appears the uncommon wave, whose phase front is inclined toward the Pointing vector to the angle, determined by relationship (15.26). In fact obtained wave is the superposition of plane wave with the phase speed $c=\sqrt{\frac{1}{\mu \varepsilon}}$ and additional wave of plane wave with the infinite phase speed orthogonal to the direction of propagation.

Let us examine one additional case, when the direction of the speed of the moving system coincides with the direction of propagation of electromagnetic wave. We will consider that there are components of the plane wave $E_{x}, H_{z}$, and also component of the speed $\pm v_{y}$. Taking into account that in this case is fulfilled the relationship $E_{x}= \pm Z_{0} H_{z}$, we obtain:

$$
\begin{aligned}
& E_{x}^{\prime}=E_{x}\left(\operatorname{ch} \frac{v_{y}}{c}-\operatorname{sh} \frac{v_{y}}{c}\right)=E_{x} \exp \left(\mp \frac{v_{y}}{c}\right), \\
& H_{z}^{\prime}=H_{z}\left(\operatorname{ch} \frac{v_{y}}{c}-\operatorname{sh} \frac{v_{y}}{c}\right)=H_{z} \exp \left(\mp \frac{v_{y}}{c}\right) .
\end{aligned}
$$

I.e. amplitudes fields on exponentially they diminish or they grow depending on direction of motion. 
The wave of the strength of an electrical (or magnetic) field of the type satisfies wave equation

$$
E(t, y)=E_{0} \sin (\omega t-k y),
$$

where $k=\frac{2 \pi}{\lambda}$ - wave number.

Upon transfer into the inertial system, which moves with the speed $\pm v_{y}$, is observed Doppler frequency shift.

The transverse Doppler effect, who long ago is discussed sufficiently, until now, did not find its confident experimental confirmation. For observing the star from moving IRS it is necessary to incline telescope on the motion of motion to the angle, determined by relationship (15.26). But in this case the star, observed with the aid of the telescope in the zenith, will be in actuality located several behind the visible position with respect to the direction of motion. Its angular displacement from the visible position in this case will be determined by relationship (15.26). But this means that this star with respect to the observer has radial it sped, determined by the relationship

$$
v_{r}=v \sin \alpha .
$$

Since for the low values of the angles $\sin \alpha \cong \alpha$, and $\alpha=\frac{v}{c}$, Doppler frequency shift will compose

$$
\omega_{d \perp}=\omega_{0} \frac{v^{2}}{c^{2}} .
$$

This result numerically coincides with results SR, but it is fundamentally different from it results in that the SR deemed that the transverse Doppler effect, defined by (15.27) actually exists, whereas in this case it is only an apparent effect.

\section{$\S 16$. The Vlasov equations and Lagrangian of the moving charge in the concept of the scalar-vector potential}

Vlasov's idea for the introduction of self-congruent field, consisted in the fact that the electromagnetic fields in the plasma and the fields, created by the charged particles must be self-consistent. This principle easily understanding based on the example of cavity resonator. Its resonance frequency determines the variable electromagnetic fields, which are subordinated to Maxwell's equations, to which are superimposed boundary conditions. And if inside the resonator appears any object, including free charges, then its resonance frequency changes in such a way that its electromagnetic fields would be coordinated with the fields by those induced by outside object. Being guided by this principle, Vlasov originally examined the system of the general equations of plasma, which include three components (electrons, ions and neutral atoms), and wrote Boltzmann equation for $s$-component of plasma in the form [11] 


$$
\frac{\partial f_{s}}{\partial t}+\operatorname{div}_{r} \vec{v} f_{s}+\frac{e_{s}}{m_{s}}(\vec{E}+[\vec{v} \times \vec{B}]) \operatorname{grad}_{v} f_{s}=\left[\frac{\partial f_{s}}{\partial t}\right]_{s 1}^{s t}+\left[\frac{\partial f_{s}}{\partial t}\right]_{s 2}^{s t}+\left[\frac{\partial f_{s}}{\partial t}\right]_{s 3}^{s t},
$$

where $f_{s}(\vec{r}, \vec{p}, t)$ - the distribution function.

The right side of the equation (16.1) represents the integrals of collisions. This system of equations included also Maxwell equations, and equation for the charge and the current, the expressed through the functions distributions. Since Vlasov was interested only in wave solutions, thus he disregarded the contributions of the integrals of collisions, since according to his estimations it left, that the frequencies of plasma waves are much more than the frequencies of the paired collisions of particles in the plasma. I.e., instead of the description of interaction of the charged particles in the plasma by means of the collisions, he proposed to use the selfcongruent field, created with the charged particles of plasma for describing the long-range potential. Instead of Boltzmann's equation Vlasov proposed to use the following system of equations for describing the charged components of plasma (electrons with the function of distributions $f_{e}(\vec{r}, \vec{p}, t)$ and positive ions with the distribution function $\left.f_{i}(\vec{r}, \vec{p}, t)\right)$ :

$$
\begin{aligned}
& \frac{\partial f_{e}}{\partial t}+\vec{v} \frac{\partial f_{e}}{\partial \vec{x}}-(e \vec{E}+e[\vec{v} \times \vec{B}]) \frac{\partial f_{e}}{\partial \vec{p}}=0 \\
& \frac{\partial f_{i}}{\partial t}+\vec{v} \frac{\partial f_{i}}{\partial \vec{x}}+(e \vec{E}+e[\vec{v} \times \vec{B}]) \frac{\partial f_{i}}{\partial \vec{p}}=0 \\
& \operatorname{rot} \vec{H}=\vec{j}+\varepsilon \frac{\partial \vec{E}}{\partial t}, \quad \operatorname{rot} \vec{E}=-\frac{\partial \vec{B}}{d t} \\
& \operatorname{div} \vec{E}=\rho, \quad \operatorname{div} \vec{B}=0 \\
& \rho=e \int\left(f_{i}-f_{e}\right) d^{3} \vec{p}, \quad \vec{j}=e \int\left(f_{i}-f_{e}\right) \vec{v} d^{3} \vec{p}
\end{aligned}
$$

In the relationship (16.2) in the first two equations in the brackets stands the force acting on the moving particle in the electrical and magnetic field, created by the surrounding charged particles. The fixed moving particles create electric field, and those moving - create magnetic field. But for writing of Vlasov equations it is possible to use the concept of scalar- vector potential, which assumes the dependence of the scalar potential of charge on the speed.

In Vlasov equations (16.2) in the first two equations, the members concluded in the brackets, present the force, which acts on the moving charge. But the concept of scalar vector potential gives the possibility to calculate this force in the system of coordinates of the moving charge, taking into account long-range forces, the surrounding charges, after excluding magnetic field [10, 18, 22]:

$$
\vec{F}=-e \sum_{j} \frac{1}{4 \pi \varepsilon} \frac{g_{j}}{r^{2}} \operatorname{ch} \frac{v_{j \perp}}{c},
$$

where $g_{j}$ - one of the external charges, which is been located at a distance $r_{j}$ from the charge $e, v_{j \perp}$ - normal component by relative charge rate with respect to the charge. 
Substituting the expression of force (16.3) in the relationships (16.2), we obtain writing of Vlasov equations in the concept of scalar- vector potential.

$$
\begin{aligned}
& \frac{\partial f_{e}}{\partial t}+\vec{v} \frac{\partial f_{e}}{\partial \vec{x}}-\left(e \sum_{j} \frac{1}{4 \pi \varepsilon} \frac{g_{j}}{r^{2}} \operatorname{ch} \frac{v_{j \perp}}{c}\right) \frac{\partial f_{e}}{\partial \vec{p}}=0 \\
& \frac{\partial f_{i}}{\partial t}+\vec{v} \frac{\partial f_{i}}{\partial \vec{x}}+\left(e \sum_{j} \frac{1}{4 \pi \varepsilon} \frac{g_{j}}{r^{2}}{ }_{j} c h \frac{v_{j \perp}}{c}\right) \frac{\partial f_{i}}{\partial \vec{p}}=0 \\
& \operatorname{rot} \vec{H}=\vec{j}+\varepsilon \frac{\partial \vec{E}}{\partial t}, \quad \operatorname{rot} \vec{E}=-\frac{\partial \vec{B}}{d t} \\
& \operatorname{div} \vec{E}=\rho, \operatorname{div} \vec{B}=0 \\
& \rho=e \int\left(f_{i}-f_{e}\right) d^{3} \vec{p}, \quad \vec{j}=e \int\left(f_{i}-f_{e}\right) \vec{v} d^{3} \vec{p}
\end{aligned} .
$$

At present Vlasov equations are the fundamental equations of the electrodynamics of the plasma, in which the electromagnetic fields are self-consistent with the fields of the charges, which present plasma. Into these equations enters the Lorentz force, which in the concept of scalar- vector potential can be expressed through the properties of the charged particles, which surround observation point. This approach, realized in this article, entirely realizes the idea of the long-range interaction of Coulomb pour on, which is the basis of Vlasov equations.

One of the methods of the solution of the problems of mechanics is Lagrange formalism. By Lagrange function or Lagrangian in the mechanics is understood the difference between the kinetic and potential energy of the system

$$
L=W_{k}(t)-W_{p}(t)
$$

If we integrate Lagrangian with respect to the time, then we will obtain the first main function Of [gamiltona], called action. Since in the general case kinetic energy depends on speeds, and potential - from the coordinates, action can be recorded as

$$
S=\int_{t_{1}}^{t_{2}} L\left(x_{i}, v_{i}\right) d t
$$

With the condition of the conservatism of this system Lagrange formalism assumes least-action principle, when system during its motion selects the way, with which the action is minimum.

In the electrodynamics Lagrangian of the charged particle, which is moved with the relativistic speed, is written as follows [4]:

$$
L=-m c^{2} \sqrt{1-\frac{v^{2}}{c^{2}}}-q\left(\varphi+\mu_{0}\left(\vec{v}_{H}\right)\right),
$$

This expression will be written down for the not relativistic speeds:

$$
L=\frac{m v^{2}}{2}-q\left(\varphi+\mu_{0}\left(\vec{v} \vec{A}_{H}\right)\right)
$$

where $q, m$ and $\vec{v}$ - charge mass and the velocity of particle, $c$ - the speed of light, $\mu_{0}$ - magnetic permeability of vacuum, $\varphi$ - the scalar potential of electric field, $\vec{A}_{H}$ - the vector potential of magnetic field, in which it moves with particle. However, the record of Lagrangian, accepted in the electrodynamics does not entirely 
satisfy the condition of the conservatism of system. The vector potential, in which moves the charge, create the strange moving charges, and the moving charge interacts not with the field of vector potential, but with the moving charges, influencing their motion. But this circumstance does not consider the existing model, since. vector potential comes out as the independent substance, with which interacts the moving charge.

In the work [15] on p. 279 the conclusion is done: "Therefore even in the relativistic approximation Lagrange's function in the electromagnetic field cannot be represented in the form differences in the kinetic and potential energy" (end of the quotation). In the relationship (16.4), of the author it confuses the term, that contains the scalar product of the charge rate and vector potential, and he does not know, to what form of energy it to carry. Landau in [14] actually also shares this point of view. The following observation is done during the introduction of the concepts of action and Lagrange function in the paragraph 16 of this work: "The following below assertions it is necessary to examine to a considerable degree as the result of experimental data. The form of action for the particle in the electromagnetic field cannot be established on the basis only of general considerations, such as the requirement of relativistic invariance. (latter it would allow in action also the member of form integral of Ads, where A scalar function)" (end of the quotation).

However, with the further consideration of this question not only are not given any experimental data, but also any theoretical substantiation of the introduction to function of Lagrange in the form (16.4). In this case the introduction of this Lagrangian leads to the theoretical problems. Without understanding the physical essence of Lagrangian, and in fact guess it's (see relationship (18.1) into [14]), the author immediately includes the potential part (the scalar product of speed and vector potential) in generalized momentum, and then for finding the force is differentiated on the coordinate of Lagrangian, calculating gradient from this value (see relationship after equality (18.1) into [14]). But, finding gradient from the work indicated, the author thus recognizes his potential status.

In the mechanics by pulse is understood the work of the mass of particle to its speed. Multiplying impulse on the speed, mechanical energy is derived. In the electrodynamics besides the appropriate angular impulse, caused by the massiveness of charge carriers, the generalized impulse is introduced

$$
\mathbf{P}=m \mathbf{v}+q \mathbf{A}
$$

in the form the sum of angular impulse and work of charge on the vector potential of magnetic field, in which moves the charge. Moreover even with the insignificant magnetic fields this additive considerably exceeds angular impulse. If generalized momentum scalar was multiplied by the speed

$$
\vec{v} \vec{P}=m(\vec{v})^{2}+q \vec{v} \vec{A}
$$

that kinetic impulse will give kinetic energy. Scalar product of speed and vector potential will also give energy, here only this energy proves to be not kinetic, but potential. Is obtained the contradictory mixing of kinetic and potential energies in 
the composition of energy of the moving charge, that also characterizes the appearing theoretical problem.

Entering (16.4) the vector potential is connected with the motion of the strange charges, with which interacts this charge. A change in the speed of the latter entails a change in the speed of strange charges, on what the energy of the moving charge is spent. As a result this record of Lagrangian does not satisfy the condition of the conservatism of system. In order to ensure the conservatism of system, it is necessary to know interaction energy of the moving charge with all strange charges, including with those, on which depends vector potential. This becomes possible with the use of a concept of the scalar- vector potential [39-40, 42, 43].

The scalar potential of the totality of strange charges at the point of the presence of this charge is equal to the sum of their scalar potentials:

$$
\varphi^{\prime}(r)=\sum_{j} \varphi\left(r_{j}\right) \operatorname{ch} \frac{v_{j \perp}}{c}=\sum_{j} \frac{1}{4 \pi \varepsilon} \frac{g_{j}}{r_{j}} \operatorname{ch} \frac{v_{j \perp}}{c} .
$$

Taking into account this circumstance Lagrangian of the charge $e$, which is found in the environment of the fixed and moving strange charges can be written down as follows:

$$
L=-e \sum_{j} \frac{1}{4 \pi \varepsilon} \frac{g_{j}}{r_{j}} \operatorname{ch} \frac{v_{j \perp}}{c} .
$$

If in selected IRS this charge $e$ moves with speed $v$, that the kinetic part, which coincides in the case of low speeds with the purely kinetic part of Lagrangian is added to this potential part, (16.5):

$$
L=\frac{m v^{2}}{2}-e \sum_{j} \frac{1}{4 \pi \varepsilon} \frac{g_{j}}{r_{j}} \operatorname{ch} \frac{v_{j \perp}}{c}
$$

In this case the values $v_{j \perp}$ make sense of the relative speeds of strange charges with respect to this charge.

The approach proposed satisfies the complete conservatism of system, since all interactions of this charge with its surrounding strange charges are considered. Relationships (16.4), (16.5) are accurate only for the case, when charge is so small that it does not influence the fields, in which it moves [13]. The record of Lagrangian (16.6) in the form excludes this limitation.

\section{$\S 17$. Transcoordinate electrodynamics in time-spatial gipercontinuum and the Mende conversion}

In the classical mechanics particle dynamics is described by the differential equations for its radius-vector, which use usual derivative of the second order on the time. Specifically, its use ensures the Galileo- invariance of equations. If we connect the set of massive material points by weightless elastic threads into the united string, i.e. fluctuation will be described by the Galileo-invariant system of differential equations. But if we complete passage to the limit, after fixing the number of 
material points to infinity, and their mass and the length of separate threads - to zero, then we will obtain the one-dimensional wave equation (equation of vibrations of string), not invariant relative to the Galileo conversions, but invariant relative to the group of pseudo-orthogonal conversions (hyperbolic turnings, which preserve pseudo-Euclidean certificate). The culprit of this strange and unexpected metamorphosis upon transfer from "material- point mechanics to continuous medium - this passage to the limit with the substitution by usual derivative to the quotient, which, generally speaking, is analytically legal [46], but it narrows the region of the physical applicability of equation. The real wave process of mechanical vibrations of string remains Galileo- invariant, but its equation is already deprived of the mathematical means of the description of passage from one inertial reference system to another, and completely ties process to one concrete frame of reference, attaching in it the ends of the string. So classics- field natural-science paradigm revealed fundamental contradiction between the continuity and the discretion [47, 48], irresistible until now, but led to the celebration in theoretical physics of the doubtful principle of the geometrization [49].

The discovery wave equation in the mechanics did not lead to the revision of ideas about the space and the time, but to this led the discovery the same equation in the electrodynamics. In the theory of relativity the corresponding group of pseudo-orthogonal conversions for the electromagnetic waves in the vacuum (conversion of Lorenz) obtained status of the subgroup of the motion of the certificate of united world physical space-time. But appears doubt about the justification of the use of traditional equations of electrodynamics, in particular, wave equation, for the adequate extraction of them of ideas about the space and the time. Easily to assume that these equations, using partial derivatives of field functions on the time, similar to the equation of mechanical fluctuations, are simply deprived of the mathematical means of the adequate description of passage from one inertial reference system to another and so completely they tie process to one concrete frame of reference. The question of the possibility of the suitable refinement or generalizing the equations of electrodynamics so arises, beginning from the equations of the induction of electric field by magnetic and magnetic=electrical. The thorough study of this problem in [10] led to the appearance of an idea about the fact that this improvement of electrodynamics must assume existence of the dependence of electromagnetic field on the speed of the motion of observer, caused not by the geometry of space-time, but by physical nature of field.

In the theory of relativity the electromagnetic field also depends on the speed of the motion of observer, but it is only defined by example through the dependence on it of the intervals of time and spatial distance (conversion of Lorenz), the relativistic invariance of electric charge occurs result of which. However, the more fundamental (direct) dependence of field on the speed is combined with the presence of this dependence even absolute value of electric charge. Until recently this not invariance of charge was confirmed only by indirect empirical data, which were being consisted in the appearance of an electric potential on the superconductive windings and the tori during the introduction in them of direct current, or in the observation of the electric pulse of the nuclear explosions [50]. 
In particular, 9 July 1962 with the explosion in space above Pacific Ocean of Hbomb with the TNT equivalent 1,4 Mt. according to the program of the USA «Starfish» the tension of electrical fields on she exceeded those forecast by Nobel laureate Bethe 1000 once. with the explosion of nuclear charge according to the program "Program $K$ ", which was realized into the USSR, the radio communication and the radar installations were also blocked at a distance to $1000 \mathrm{~km}$. It was discovered, that the registration of the consequences of space nuclear explosion was possible at the large (to 10 thousand kilometers) distances from the point of impact. The electric fields of pulse led to the large focusing to the power cable in the lead shell, buried at the depth about $1 \mathrm{~m}$, which connects power station in Akmola with Alma-Ata. Focusing was so great that the automation opened cable from the power station.

However, 2015 was marked by obtaining the already direct experimental confirmation of this phenomenon as a result of detection and study of the pulse of the electric field, which appears with the warming-up of the plasma as a result of the discharge through the dischargers of the capacitors of the great capacity [50]. It turned out that in the process of the warming-up of plasma with an equal quantity in it of electrons and positive ions in it the unitary negative charge of free electrons, not compensated by slower positive ions, is formed.

This fact contradicts not only the classical, but also relativistic conversions of electromagnetic field upon transfer from one inertial reference system to another, testifying about the imperfection not only of classical, but also relativistic ideas about the space and the time. Idea about the fact that the promising electrodynamics must assume existence of the dependence of electromagnetic field on the speed of the motion of observer, caused not by the geometry of space-time, and by physical nature of field, which does not assume the invariance of electric charge, was developed in a number of the work of F. F. Mende, beginning [10]. In these works, in particular, in $[10,50]$ is given the substantiation of introduction into the electrodynamics instead of the classical and relativistic new conversions of electromagnetic field, which was called the conversions of Mende.

However, the sequential development of this radical idea, as not the invariance of charge, requires the deep revision of the mathematical apparatus for electrodynamics, called to the creation of the mathematical means of the more adequate description of passage from one inertial reference system to another. Approach to precisely this development of the mathematical apparatus for electrodynamics was proposed by A.S. Dubrovin in [51]. This approach lies within the framework the sequential revision of ideas about the space and the time with the failure of the relativistic and the passage to the new ideas, which we call gipercontinual.

The concept of time-spatial gipercontinual is introduced in [52] as a result the joint study of the algebraic and geometric structures of the commutative algebras with one, elements of which are the functions of sine waves. The hypothesis of gipercontinual (about the hierarchical gipercontinual structure of world physical space-time) is starting point of scientific studies, directed toward the generalization of ideas about the structure of space and time in the course of passage from the contemporary quantum scientific paradigm to the new system, that simultaneously 
structurally connecting up its framework continuity and the discretion, dynamicity and static character, and also globality and the locality [47, 48, 53]. The hierarchical quality of gipercontinual limits the applicability of the conventional principle of geometrization in physics and the connected with it ideas of symmetry in the geometry due to the introduction into theoretical physics of the ideas of hierarchical quality $[49,54]$, effectiveness of which have approved we with the creation of the standard model of the protected automated system (EMZAS) and the mathematical apparatus of the EMZAS- networks [55].

In [51] is proposed new approach to the development of the mathematical apparatus for electrodynamics in the direction of the more adequate description of passage from one inertial reference system to another on the basis of gipercontinual ideas about the space and in the time due to the improvement of differential calculus of the field functions under the assumption of their dependence on the speed of the motion of observer. Let us describe this new approach in more detail in accordance with [51].

Two inertial reference systems with the time united for them will examine $t \in \mathbb{R}$. One of them (with the system of rectangular Cartesian space coordinates $O X Y Z$ ) let us name laboratory (not prime) and we will interpret it as relatively fixed. The second (with the system of rectangular Cartesian space coordinates $O^{\prime} X^{\prime} Y^{\prime} Z^{\prime}$ ) let us name substantive (prime) and we will interpret it as connected with the certain moving real or imaginary medium. Let us assume that with $t=0$ the system of space coordinates of both frames of references they coincide. Let us introduce the indices $\alpha=\overline{1,3}, \beta=\overline{1,3}$. Coordinates along the axes $O X, O Y, O Z, O^{\prime} X^{\prime}, O^{\prime} Y^{\prime}$, $O^{\prime} Z^{\prime}$ we will assign by variables $x^{\alpha}$ and $x^{\prime \alpha}$ respectively. Unit vectors along the axes $O X$ and $O^{\prime} X^{\prime}$, the axes $O Y$ and $O^{\prime} Y^{\prime}$, the axes $O Z, O^{\prime} Z^{\prime}$ let us designate through $\mathbf{e}_{\beta}=\left(e_{\beta}^{\alpha}\right)$, moreover $e_{\beta}^{\alpha}=\delta_{\alpha \beta}$, where $\delta_{\alpha \beta}-$ Kronecker symbol. Through $\mathbf{v}=\left(v^{\alpha}\right), v$ let us designate the velocity vector of the motion of substantive frame of reference relative to laboratory and the module of this vector. Directing a unit vector $\mathbf{e}_{1}$ lengthwise $\mathbf{v}$, we have: $\mathbf{v}=v \mathbf{e}_{1}=\left(v^{\alpha}\right), v^{\alpha}=v \delta_{\alpha 1}$. Event in the data two frame of references takes the form $\mathbf{x}=(\mathbf{r}, t)=\left(x^{\alpha}, t\right)$; $\mathbf{x}^{\prime}=\left(\mathbf{r}^{\prime}, t\right)=\left(x^{\prime \alpha}, t\right)$, where $\mathbf{r}=\left(x^{\alpha}\right), \mathbf{r}^{\prime}=\left(x^{\prime \alpha}\right)-$ the radius-vectors. We will consider that the physical equivalence of events $\mathbf{x}, \mathbf{x}^{\prime}$ indicates the validity of the conversion of Galileo

$$
\mathbf{r}=\mathbf{r}^{\prime}+t \mathbf{v}
$$

or, otherwise, substituting vector idea by the component,

$$
x^{\alpha}=x^{\prime \alpha}+t v \delta_{\alpha 1}
$$

Classical physical field is described in the laboratory and substantive frame of references by its field functions $\Phi(\mathbf{r}, t)$ and $\Phi^{\prime}\left(\mathbf{v}, \mathbf{r}^{\prime}, t\right)$, moreover $\Phi^{\prime}\left(\mathbf{0}, \mathbf{r}^{\prime}, t\right)=\Phi\left(\mathbf{r}^{\prime}, t\right)$, and equality $\mathbf{v}=0$ indicates $v^{\alpha}=0$. Their values are 
called field variables. For fields on different physical nature they can be suitable the different mathematical ideas of field functions, so that field variables can be, for example, scalar or vector with the material or complex values of their most variable or vector components. If in the role of this field electric field comes out, then in this role can come out the functions of its tension $\mathbf{E}=\Phi(\mathbf{r}, t), \mathbf{E}^{\prime}=\Phi^{\prime}\left(\mathbf{v}, \mathbf{r}^{\prime}, t\right)$ and in the case of magnetic field we have functions of the magnetic induction $\mathbf{B}=\Phi(\mathbf{r}, t), \quad \mathbf{B}^{\prime}=\Phi^{\prime}\left(\mathbf{v}, \mathbf{r}^{\prime}, t\right)$.

In the classical nonrelativistic field theory it is considered that the equality occurs

$$
\Phi\left(\mathbf{r}^{\prime}+t \mathbf{v}, t\right)=\Phi^{\prime}\left(\mathbf{v}, \mathbf{r}^{\prime}, t\right)
$$

mathematically expressing the physical concept of the invariance of field relative to the speed of the motion of observer.

In the theory of relativity (17.3) no longer it is carried out, but the Lorenz conversions are used instead of the Galileo conversions. But this not invariance of field does not have fundamental, that not connected with the geometry of the space-time of physical nature, but it occurs simply the consequence of the effects of the reduction of lengths and time dilation in the moving frame of references. The proposed by us giperkontinuum ideas about the space and the time [51] provide for the great possibilities of the invariance of various physical processes relative to various transformation groups of coordinates with the fact that special role in time-spatial giperkontinuum play the Galileo conversions (17.1), since they in this case they treat as the level Lorenz conversions of infinitely high level and, thus, they make it possible in a united manner to synchronize all events in all separate continua, hierarchically structuring into united giperkontinuum. Natural to consider that in giperkontinuum the field also not is invariant relative to the speed of the motion of observer, but to explain this by the already fundamental properties of field, not connected with the geometry of separate continua.

Arises the question about the possible versions of complete differentiation concerning the time of field function in the laboratory frame of reference $\Phi(\mathbf{r}, t)$, of that produced depending on substantive frame of reference. In fluid mechanics and classical mechanics widely is used the derivative of Lagrange (the substantional derivative), which has the same arguments as the initial field function:

$$
\frac{d \Phi(\mathbf{r}, t)}{d t}=\frac{d \Phi\left(\mathbf{r}^{\prime}+t \mathbf{v}, t\right)}{d t}=\lim _{\Delta t \rightarrow 0} \frac{\Phi\left(\mathbf{r}^{\prime}+(t+\Delta t) \mathbf{v}, t+\Delta t\right)-\Phi\left(\mathbf{r}^{\prime}+t \mathbf{v}, t\right)}{\Delta t} .
$$

But it is possible to examine also the derivative (let us name its derivative of Galileo), whose arguments will coincide with the arguments of field function no longer in the laboratory, but in the substantive frame of reference:

$$
\frac{\partial^{\prime} \Phi}{\partial t}\left(\mathbf{v}, \mathbf{r}^{\prime}, t\right)=\frac{d \Phi\left(\mathbf{r}^{\prime}+t \mathbf{v}, t\right)}{d t}=\lim _{\Delta t \rightarrow 0} \frac{\Phi\left(\mathbf{r}^{\prime}+(t+\Delta t) \mathbf{v}, t+\Delta t\right)-\Phi\left(\mathbf{r}^{\prime}+t \mathbf{v}, t\right)}{\Delta t},
$$

If the arguments of the derivatives of Lagrange and Galileo are connected with equality (17.1), that their corresponding values are equal and are decomposed into one and the same sum of quotient on the time and the convective derivative of field function in the laboratory frame of reference: 


$$
\frac{\partial^{\prime} \Phi}{\partial t}\left(\mathbf{v}, \mathbf{r}^{\prime}, t\right)=\frac{d \Phi(\mathbf{r}, t)}{d t}=\frac{\partial \Phi\left(\mathbf{r}^{\prime}+t \mathbf{v}, t\right)}{\partial t}+(\mathbf{v} \cdot \nabla) \Phi\left(\mathbf{r}^{\prime}+t \mathbf{v}, t\right)
$$

Let us explain a difference in the physical sense of the Lagrange and Galilean derivatives of field function. Lagrange's derivative (17.4) is complete time derivative of the function of field in the laboratory frame of reference, measured at the point of space, which in the laboratory frame of reference at the moment of time $t$ has a radius-vector $\mathbf{r}$, determined by the equality (17.1). But Galileo's derivative (17.5) is complete time derivative of the function of field in the laboratory frame of reference, measured at the point of space, which in the substantive frame of reference has a radius-vector $\mathbf{r}^{\prime}$. The concepts of Lagrange and Galilean derivatives (17.4)(17.6) naturally are generalized to the case derivative of higher order $(n=\overline{1, \infty})$ :

$$
\begin{gathered}
\frac{d^{1} \Phi(\mathbf{r}, t)}{d t^{1}}=\frac{d \Phi(\mathbf{r}, t)}{d t} ; \frac{d^{n+1} \Phi(\mathbf{r}, t)}{d t^{n+1}}=\frac{d}{d t} \frac{d^{n} \Phi(\mathbf{r}, t)}{d t^{n}} \\
\frac{\partial^{\prime 1} \Phi}{\partial t^{1}}\left(\mathbf{v}, \mathbf{r}^{\prime}, t\right)=\frac{\partial^{\prime} \Phi}{\partial t}\left(\mathbf{v}, \mathbf{r}^{\prime}, t\right) ; \frac{\partial^{\prime n} \Phi}{\partial t^{n}}\left(\mathbf{v}, \mathbf{r}^{\prime}, t\right)=\frac{d^{n} \Phi(\mathbf{r}, t)}{d t^{n}} .
\end{gathered}
$$

Within the framework concepts of the invariance of field relative to the speed of the motion of observer, i.e., with fulfillment condition (17.3), we have:

$$
\frac{\partial^{\prime} \Phi}{\partial t}\left(\mathbf{v}, \mathbf{r}^{\prime}, t\right)=\frac{d \Phi\left(\mathbf{r}^{\prime}+t \mathbf{v}, t\right)}{d t}=\frac{d \Phi^{\prime}\left(\mathbf{v}, \mathbf{r}^{\prime}, t\right)}{d t}=\frac{\partial \Phi^{\prime}\left(\mathbf{v}, \mathbf{r}^{\prime}, t\right)}{\partial t},
$$

i.e., Galilean the derivative of field in the laboratory frame of reference is not distinguished from the particular time derivative of the function of field in the substantive frame of reference. Therefore introduction within the framework to this concept of the derivative of Galileo as some new mathematical object with its independent physical sense, is superfluous. However, within the framework relativistic ideas examination by Galileo's derivative is empty because of the emptiness of very Galileo conversions (in contrast to the Lorenz conversions). But giperkontinual ideas about the space and the time make Galilean derived completely by that claimed, and equality (17.7) - to false.

This view on the space, the period and the electromagnetic field in conjunction with the application of Galileo's derivative leads to the new, trans-coordinate formulation of the electrodynamics [51]. It generalizes the conventional formulation of Hertz-Heaviside, which will be examined below.

Electromagnetic field in the isotropic homogeneous medium without the dispersion is described in the laboratory and substantive frame of references by its variables (tension of electric field $\mathbf{E}=\left(E^{\alpha}\right), \mathbf{E}^{\prime}=\left(E^{\prime \alpha}\right)$ and magnetic induction $\mathbf{B}=\left(B^{\alpha}\right)$, $\mathbf{B}^{\prime}=\left(B^{\prime \alpha}\right)$ ), by constants (electrical $\varepsilon_{0}$ and magnetic $\mu_{0}$, and also expressed as them speed of light in the vacuum $c=1 / \sqrt{\varepsilon_{0} \mu_{0}}$ ), by the parameters (dielectric and magnetic constant $\varepsilon$ and $\mu$, and also the density of strange electric charge $\rho, \rho^{\prime}$, the electric current density of conductivity $\mathbf{j}=\left(j^{\alpha}\right), \mathbf{j}^{\prime}=\left(j^{\prime \alpha}\right)$, electric charge $Q$, $Q^{\prime}$, electric current $\left.I, I^{\prime}\right)$, by field functions $\mathbf{E}=\mathbf{E}(\mathbf{r}, t)=\left(E^{\alpha}(\mathbf{r}, t)\right)$, 


$$
\begin{aligned}
& \mathbf{B}=\mathbf{B}(\mathbf{r}, t)=\left(B^{\alpha}(\mathbf{r}, t)\right), \mathbf{E}^{\prime}=\mathbf{E}^{\prime}\left(v, \mathbf{r}^{\prime}, t\right)=\left(E^{\prime \alpha}\left(v, \mathbf{r}^{\prime}, t\right)\right), \\
& \mathbf{B}^{\prime}=\mathbf{B}^{\prime}\left(v, \mathbf{r}^{\prime}, t\right)=\left(B^{\prime \alpha}\left(v, \mathbf{r}^{\prime}, t\right)\right), \text { moreover } \\
& \mathbf{E}^{\prime}\left(0, \mathbf{r}^{\prime}, t\right)=\mathbf{E}\left(\mathbf{r}^{\prime}, t\right) ; \mathbf{B}^{\prime}\left(0, \mathbf{r}^{\prime}, t\right)=\mathbf{B}\left(v, \mathbf{r}^{\prime}, t\right) .
\end{aligned}
$$

In the classical nonrelativistic electrodynamics it is relied:

$$
\mathbf{E}\left(\mathbf{r}^{\prime}+t v \mathbf{e}_{1}, t\right)=\mathbf{E}^{\prime}\left(v, \mathbf{r}^{\prime}, t\right) ; \mathbf{B}\left(\mathbf{r}^{\prime}+t v \mathbf{e}_{1}, t\right)=\mathbf{B}^{\prime}\left(v, \mathbf{r}^{\prime}, t\right),
$$

what is the application of a general formula (17.3) of the invariance of field relative to the speed of the motion of observer for the case of electromagnetic field. The proposed by us giperkontinual ideas about the space and the time [52] exceed the scope of this concept, but is explained nature of this not invariance not by the geometry of united space-time similar to the theory of relativity, but by the fundamental properties of field.

The integral form of Maxwell equations in the idea of Hertz- Heaviside with the above-indicated conditions (isotropy, the uniformity of medium, the absence in it of dispersion) is the following system of four integral equations of the electrodynamics:

$$
\begin{aligned}
\underset{s}{\int} \mathbf{E} \cdot d s=\mathrm{Q} /\left(\varepsilon \varepsilon_{0}\right) ; \underset{s}{ } \mathbf{B} \cdot d s=0 ; \underset{l}{\int} \mathbf{E} \cdot d l=-\frac{d}{d t} \int_{s} \mathbf{B} \cdot d s ; \\
\frac{c^{2}}{\varepsilon \mu} \int_{l} \mathbf{B} \cdot d l=\frac{\mathrm{I}}{\varepsilon \varepsilon_{0}}+\frac{d}{d t} \int_{s} \mathbf{E} \cdot d s,
\end{aligned}
$$

where $s, l-$ the arbitrary two-dimensional closed (for the first two equations) or open (for the second two equations) surface and its limiting locked outline, which not compulsorily coincides with the electric circuit.

If we on Medium put the even additional condition of the absence of free charges and currents, then last two equations (17.10) will take the form:

$$
\iint_{l} E \cdot d l=-\frac{d}{d t} \int_{s} B \cdot d s, \quad \int_{l} B \cdot d l=\frac{\varepsilon \mu}{c^{2}} \frac{d}{d t} \int_{s} E \cdot d s .
$$

They are the integral form of the law of the induction of Faraday and circulation theorem of magnetic field in the laboratory frame of reference for this special case of medium.

These two laws take the mutually symmetrical form with an accuracy to of scalar factor, by virtue of which their analysis it is identical. Let us examine the first law in more detail, for example. In Faraday's experiences it is experimentally established that in the outline the identical currents appear regardless of the fact, this outline relative to the current carrying outline does move or it rests, and the current carrying outline moves, provided their relative motion in both cases was identical (Galilean invariance of Faraday law). Therefore the flow through the outline can change as a result of a change of the magnetic field with time, and the position of its boundary also because with the displacement of outline changes [56]. The corresponding generalization of laws (17.11) to the case of the outline, which moves in the laboratory and which is rested in the substantive frame of reference, takes the form: 


$$
\iint_{l} E^{\prime} \cdot d l=-\frac{d}{d t} \int_{s} B \cdot d s, \int_{l} B^{\prime} \cdot d l=\frac{\varepsilon \mu}{c^{2}} \frac{d}{d t} \int_{s} E \cdot d s,
$$

where $E^{\prime}, B^{\prime}$ are described fields in the element $d l$ in the substantive frame of reference, i.e., in such inertial reference system, in which $d l$ it rests; specifically, such electric field causes the appearance of a current in the case of the presence of real electric circuit in this place. Equations (17.12) are completely interesting and uncommon from a mathematical point of view, since they mutually connect field variables in the different inertial reference systems (let us name such equations trans-coordinate). Specifically, the use of trans-coordinate equations makes it possible to adequately describe physical fields in giperkontinuum. At the same time in this case the discussion deals not simply about the trans-coordinate of equations (17.12), and with their global trans-coordinate, ensured by use by the Galilean derivative (connected by them inertial reference systems they can move relative to each other with the arbitrary speed, and not compulsorily with infinitely small).

Returning to the system of equations (17.10), it is possible to establish that the region of its applicability is limited by the requirement of the state of rest of outline $l$ in the laboratory frame of reference. If we remove this limitation, after requiring only the states of rest of outline $l$ in the substantive frame of reference, then will come out the known idea of Maxwell equations (we call his transcoordinate [51]), integral form of which will be in it the system of the generalizing (17.10) four integral equations of the electrodynamics of the moving media:

$$
\begin{gathered}
\int_{s} \mathbf{E} \cdot d s=\mathrm{Q} /\left(\varepsilon \varepsilon_{0}\right) ; \int_{s} \mathbf{B} \cdot d s=0 ; \underset{l}{\int_{l} \mathbf{E}^{\prime} \cdot d l=-\frac{d}{d t} \int_{s} \mathbf{B} \cdot d s ;} \\
\frac{c^{2}}{\varepsilon \mu} \int_{l} \mathbf{B}^{\prime} \cdot d l=\frac{\mathrm{I}^{\prime}}{\varepsilon \varepsilon_{0}}+\frac{d}{d t} \int_{s} \mathbf{E} \cdot d s .
\end{gathered}
$$

If the trans-coordinate idea of the Maxwell equations (both in that examined by integral and in that examined lower than the differential forms) to interpret in the context of the description of electromagnetic field in time-spatial giperkontinuum, then it is necessary to consider that the equalities (17.8) are always carried out, but (17.9) - in the general case no.

Equations (17.12), (17.13) are known in the classical electrodynamics [56, 57]. Arises question, as to pass from the equations in the integral form (17.12) and (17.13) to the corresponding to equations in the differential form adequate of physical reality by means.

The differential form of Maxwell equations in the idea of Hertz-Heaviside is following system of four of those corresponding to the integral equations (17.10) of the differential equations of electrodynamics, which relate to the laboratory frame of reference:

$$
\nabla \cdot \mathbf{E}=\rho /\left(\varepsilon \varepsilon_{0}\right) ; \quad \nabla \cdot \mathbf{B}=0 ; \quad \nabla \times \mathbf{E}=-\partial \mathbf{B} / \partial t ;
$$




$$
\nabla \times \mathbf{B}=\mu \mu_{0} \mathbf{j}+\left(\varepsilon \mu / c^{2}\right)(\partial \mathbf{E} / \partial t)
$$

Equations (17.14) traditionally successfully are used in the electrodynamics, but, as it will be shown below, in this case have essential deficiency - the field of their of applicability it is limited by the case of agreeing the laboratory and substantive frame of references $(v=0)$, i.e., these equations they are deprived of the mathematical means of the adequate description of passage from one inertial reference system to another, completely tying process to one (laboratory frame of reference).

In [56] based on the example of Farrday law is formulated the following approach to the passage from the integral to the differential form of equations electrodynamics: "Faraday law can be written down also in the differential form, if we use ourselves the Stokes' theorem and to consider outline as that being resting in the selected frame of reference (so that $E$ and $B$ they would be determined in one and the same frame of reference)". This approach answers the concept of the invariance of physical field relative to the speed of the motion of observer, assuming simple failure of the trans-coordinate of equations by means of the application (17.9). But if we reject this concept, then we must reject this approach. Thus, the differential form of the corresponding equations must be the same trans-coordinate as integral (17.12) and (17.13).

In accordance with the given traditional approach, in [57] is introduced the operation of differentiation with respect to time in the moving (substantive) frame of reference, designated there through $\partial^{\prime} / \partial t$. In this case it is secretly assumed that at the point of space, which in the substantive frame of reference has a radius-vector $\mathbf{r}^{\prime}$, measurement by field variable in the laboratory frame of reference equivalent to its measurement in the same substantive frame of reference. But these measurements are not equivalent out of the concept of the invariance of physical field relative to the speed of the motion of observer. Therefore measurement must be limited by laboratory frame of reference, not carry its results for the substantive. Thus, we come to the derivative of Galileo (17.5), of the electrodynamics in the differential form leaving equations globally trans-coordinate.

The unknown globally trans-coordinate differential equations of electrodynamics, which correspond to integral equations (17.12) and which use Galilean derivative, take the form:

$$
\nabla \times E^{\prime}=-\frac{\partial^{\prime} B}{\partial t}, \nabla \times B^{\prime}=\frac{\varepsilon \mu}{c^{2}} \frac{\partial^{\prime} E}{\partial t} .
$$

They are generalization to the case of the no coincidence of the laboratory and substantive frame of references $(\mathbf{v} \neq \mathbf{0})$ of the known differential Maxwell equations:

$$
\nabla \times E=-\frac{\partial B}{\partial t}, \nabla \times B=\frac{\varepsilon \mu}{c^{2}} \frac{\partial E}{\partial t} .
$$

The differential form of Maxwell equations in the trans-coordinate idea for the case of isotropic, homogeneous medium without the dispersion is the following system of four new globally trans-coordinate differential equations of the electrodynamics: 


$$
\begin{gathered}
\nabla \cdot \mathbf{E}(\mathbf{r}, t)=\frac{\rho(\mathbf{r}, t)}{\varepsilon \varepsilon_{0}} ; \nabla \cdot \mathbf{B}(\mathbf{r}, t)=0 ; \\
\nabla \times \mathbf{E}^{\prime}\left(v, \mathbf{r}^{\prime}, t\right)=-\frac{\partial^{\prime} \mathbf{B}}{\partial t}\left(v, \mathbf{r}^{\prime}, t\right) ; \nabla \times \mathbf{B}^{\prime}\left(v, \mathbf{r}^{\prime}, t\right)=\mu \mu_{0} \mathbf{j}^{\prime}\left(v, \mathbf{r}^{\prime}, t\right)+\frac{\varepsilon \mu}{c^{2}} \frac{\partial^{\prime} \mathbf{E}}{\partial t}\left(v, \mathbf{r}^{\prime}, t\right),
\end{gathered}
$$

where $\partial^{\prime} \mathbf{E} / \partial t, \partial^{\prime} \mathbf{B} / \partial t$ - the derivatives of Galileo of field functions, expressed as particular time derivatives and convective derivatives of the same field functions in the laboratory frame of reference by the following equalities:

$$
\begin{aligned}
& \frac{\partial^{\prime} \mathbf{E}}{\partial t}\left(v, \mathbf{r}^{\prime}, t\right)=\frac{\partial \mathbf{E}\left(\mathbf{r}^{\prime}+t v \mathbf{e}_{1}, t\right)}{\partial t}+\left(v \mathbf{e}_{1} \cdot \nabla\right) \mathbf{E}\left(\mathbf{r}^{\prime}+t v \mathbf{e}_{1}, t\right) ; \\
& \frac{\partial^{\prime} \mathbf{B}}{\partial t}\left(v, \mathbf{r}^{\prime}, t\right)=\frac{\partial \mathbf{B}\left(\mathbf{r}^{\prime}+t v \mathbf{e}_{1}, t\right)}{\partial t}+\left(v \mathbf{e}_{1} \cdot \nabla\right) \mathbf{B}\left(\mathbf{r}^{\prime}+t v \mathbf{e}_{1}, t\right) .
\end{aligned}
$$

With $v=0$ (17.17), (17.18) it passes in (17.14).

In the particular case the absences of free charges and currents of equation (17.17), (17.18) will take the form:

$$
\begin{gathered}
\nabla \cdot \mathbf{E}(\mathbf{r}, t)=0 ; \nabla \cdot \mathbf{B}(\mathbf{r}, t)=0 ; \\
\nabla \times \mathbf{E}^{\prime}\left(v, \mathbf{r}^{\prime}, t\right)=-\frac{\partial^{\prime} \mathbf{B}}{\partial t}\left(v, \mathbf{r}^{\prime}, t\right) ; \nabla \times \mathbf{B}^{\prime}\left(v, \mathbf{r}^{\prime}, t\right)=\frac{\varepsilon \mu}{c^{2}} \frac{\partial^{\prime} \mathbf{E}}{\partial t}\left(v, \mathbf{r}^{\prime}, t\right) .
\end{gathered}
$$

With $v=0$ (17.21), (17.22) it passes into the following well-known system of Maxwell equations:

$$
\begin{gathered}
\nabla \cdot \mathbf{E}(\mathbf{r}, t)=0 ; \nabla \cdot \mathbf{B}(\mathbf{r}, t)=0 ; \nabla \times \mathbf{E}(\mathbf{r}, t)=-\frac{\partial \mathbf{B}(\mathbf{r}, t)}{\partial t} ; \\
\nabla \times \mathbf{B}(\mathbf{r}, t)=\frac{\varepsilon \mu}{c^{2}} \frac{\partial \mathbf{E}(\mathbf{r}, t)}{\partial t} .
\end{gathered}
$$

By the vector product of nabla to both parts of the equations (17.16) with their mutual substitution into each other obtains the known wave differential equations

$$
c^{2} \nabla^{2} E=\varepsilon \mu \frac{\partial^{2} E}{\partial t^{2}}, c^{2} \nabla^{2} B=\varepsilon \mu \frac{\partial^{2} B}{\partial t^{2}} .
$$

The absence of trans-coordinate is their drawback, they are valid only in the case of agreeing the laboratory and substantive frame of references ( $\mathbf{v}=\mathbf{0})$. It is analogous, i.e., by the vector product of nabla to both parts of the equations (17.15) with their mutual substitution into each other, we will obtain the new equations of electrodynamics - the globally trans-coordinate wave differential equations, which use Galilean derivative of field functions and generalizing equations (17.24) in the case $\mathbf{v} \neq 0$ :

$$
c^{2} \nabla^{2} E^{\prime}=\varepsilon \mu \frac{\partial^{\prime 2} E}{\partial t^{2}}, c^{2} \nabla^{2} B^{\prime}=\varepsilon \mu \frac{\partial^{\prime 2} B}{\partial t^{2}} .
$$

We investigate in more detail the equation of form (17.25) in connection with to arbitrary field functions $\Phi(x, t)$, also, $\Phi^{\prime}\left(v, x^{\prime}, t\right)$ for the case of plane wave with the wave vector, collinear to vector $\mathbf{v}=(v, 0,0)$ and to axes $O X, O^{\prime} X^{\prime}$, coordi- 
nates along which are assigned by the variables $x, x^{\prime}$. In this case the equation proves to be one-dimensional, and field functions - scalar:

$$
c^{2} \frac{\partial^{2}}{\partial x^{\prime 2}} \Phi^{\prime}\left(v, x^{\prime}, t\right)=\varepsilon \mu \frac{\partial^{\prime 2} \Phi}{\partial t^{2}}\left(v, x^{\prime}, t\right)=\varepsilon \mu \frac{d^{2}}{d t^{2}} \Phi\left(x^{\prime}+v t, t\right) .
$$

If we differentiate in the right side (17.26), this equation of signs the form:

$$
\begin{aligned}
& \frac{c^{2}}{\varepsilon \mu} \frac{\partial^{2}}{\partial x^{\prime 2}} \Phi^{\prime}\left(v, x^{\prime}, t\right)=\left(\frac{\partial^{2}}{\partial t^{2}}+2 v \frac{\partial^{2}}{\partial t \partial x}+v^{2} \frac{\partial^{2}}{\partial x^{2}}\right) \Phi\left(x^{\prime}+v t, t\right)= \\
& \left(\frac{\partial}{\partial t}+v \frac{\partial}{\partial x}\right)^{2} \Phi\left(x^{\prime}+v t, t\right)
\end{aligned}
$$

With $v=0$ (17.26), (17.27) it degenerates into the one-dimensional version of the wave equation of the form (17.24):

$$
c^{2} \frac{\partial^{2}}{\partial x^{2}} \Phi(x, t)=\varepsilon \mu \frac{\partial^{2}}{\partial t^{2}} \Phi(x, t)
$$

Any solution (17.28) is determined by the proper superposition of the simple harmonic waves

$$
\Phi(x, t)=A \cos \left(\omega t-k_{x} x+\phi\right)
$$

with the approximate values of the parameters $A \geq 0, \omega>0, k_{x} \neq 0, \phi \in \mathbb{R}-$ amplitude, angular frequency, the projection of wave vector on the axis $O X$ and the initial phase of wave. In this case all waves (17.29) must have one and the same phase speed $\omega / k=c / \sqrt{\varepsilon \mu}$, where $k=\left|k_{x}\right|$ - wave number. We will search for function $\Phi^{\prime}\left(v, x^{\prime}, t\right)$, satisfying (17.26)-(17.29), also in the form of simple harmonic wave, but with those depending on $v$ by the parameters $A^{\prime}(v), \omega^{\prime}(v), k_{x}^{\prime}(v), \phi^{\prime}(v)$ :

$$
\Phi^{\prime}\left(v, x^{\prime}, t\right)=A^{\prime}(v) \cos \left(\omega^{\prime}(v) t-k_{x}^{\prime}(v) x^{\prime}+\phi^{\prime}(v)\right),
$$

$\Phi^{\prime}\left(0, x^{\prime}, t\right)=\Phi\left(x^{\prime}, t\right), A^{\prime}(0)=A, \omega^{\prime}(0)=\omega, k_{x}^{\prime}(0)=k_{x}, \phi^{\prime}(0)=\phi$. Let us substitute (17.29), (17.30) in (17.27):

$$
\begin{aligned}
& c^{2} k_{x}^{\prime 2}(v) A^{\prime}(v) \cos \left(\omega^{\prime}(v) t-k_{x}^{\prime}(v) x^{\prime}+\phi^{\prime}(v)\right)= \\
& \varepsilon \mu\left(\omega-k_{x} v\right)^{2} A \cos \left(\omega t-k_{x}\left(x^{\prime}+v t\right)+\phi\right)
\end{aligned}
$$

Equalizing the similar parameters of wave on the left side (17.31) and waves in the right, we have:

$$
\begin{gathered}
A^{\prime}(v)=\left(\operatorname{sgn} k_{x}-\frac{\sqrt{\varepsilon \mu}}{c} v\right)^{2} A, \omega^{\prime}(v)=\left|\omega-k_{x} v\right|=\left|1-\frac{\sqrt{\varepsilon \mu}}{c} v \operatorname{sgn} k_{x}\right| \omega, \quad \\
k_{x}^{\prime}(v)=k_{x} \operatorname{sgn}\left(\omega-k_{x} v\right), k^{\prime}(v)=\left|k_{x}^{\prime}(v)\right|=k, \phi^{\prime}(v)=\phi \operatorname{sgn}\left(\omega-k_{x} v\right),\left|\phi^{\prime}(v)\right|=|\phi| .
\end{gathered}
$$


Thus, upon transfer from the laboratory to the substantive frame of reference change amplitude and frequency (17.32) of simple harmonic wave, and its wave number and module of initial phase (17.33) remain constant. In this case the frequency changes in such a way that phase wave velocity in the substantive frame of reference is obtained according to the classical summation rule of speeds from its phase speed in the laboratory frame of reference and speed of substantive frame of reference relative to the laboratory:

$$
\begin{gathered}
\omega^{\prime}(v) / k_{x}^{\prime}(v)=\omega^{\prime}(v) / k_{x}=\omega / k_{x}-v, \\
\omega^{\prime}(v) / k^{\prime}(v)=\left|\omega / k-v \operatorname{sgn} k_{x}\right|=\left|c / \sqrt{\varepsilon \mu}-v \operatorname{sgn} k_{x}\right|
\end{gathered}
$$

From (17.32)-(17.34) it is evident that if the vector of phase wave velocity in the laboratory frame of reference coincides with the velocity vector of substantive frame of reference in it $\left(k_{x}>0, v=\omega / k\right)$, that in the substantive frame of reference wave generally disappears $\left(A^{\prime}(v)=0\right)$. Thus, in contrast to the theory of relativity, in the theory of gipercontinual this wave always can be destroyed by the suitable selection of frame of reference. But if relative to laboratory frame of reference substantial frame of reference outdistances wave, then upon transfer from the laboratory frame of reference to the substantive the direction of propagation of wave changes by the opposite. If in the laboratory frame of reference wave is propagated in the positive direction, then upon transfer into the substantive it will satisfy wave equation (17.35), while if in the negative, then to the equation (17.36):

$$
\begin{aligned}
& (c / \sqrt{\varepsilon \mu}-v)^{2} \partial^{2} \Phi^{\prime}\left(v, x^{\prime}, t\right) / \partial x^{\prime 2}=\partial^{2} \Phi^{\prime}\left(v, x^{\prime}, t\right) / \partial t^{2} ; \\
& (c / \sqrt{\varepsilon \mu}+v)^{2} \partial^{2} \Phi^{\prime}\left(v, x^{\prime}, t\right) / \partial x^{\prime 2}=\partial^{2} \Phi^{\prime}\left(v, x^{\prime}, t\right) / \partial t^{2} .
\end{aligned}
$$

The selection of inertial reference system to the role of laboratory is, generally speaking, conditional. Thus, substantial frame of reference it is possible in turn to accept for the laboratory, and in the role of substantial to examine certain by third (twice prime) inertial reference system with that directed to the same side, that also $O X, O^{\prime} X^{\prime}$, by attitude reference $O^{\prime \prime} X^{\prime \prime}$, the coordinate along which is assigned by the variable $x^{\prime \prime}$. Let, for example, the point $O^{\prime \prime}$ move in the positive direction of axis $O^{\prime} X^{\prime}$ with the speed $\Delta v$. Wave in the new laboratory and substantive frame of references will have an identical wave number and a module of initial phase and will be described by field functions $\Phi^{\prime}\left(v, x^{\prime}, t\right)$ and $\Phi^{\prime}\left(v+\Delta v, x^{\prime \prime}, t\right)$ respectively. The role of equation (17.28) will play (17.35) or (17.36), the role of the function of wave (17.29) - function (17.30), while the role of equations (17.35), (17.36) - the following wave equations:

$$
\begin{aligned}
& (c / \sqrt{\varepsilon \mu}-(v+\Delta v))^{2} \partial^{2} \Phi^{\prime}\left(v+\Delta v, x^{\prime \prime}, t\right) / \partial x^{\prime \prime 2}=\partial^{2} \Phi^{\prime}\left(v+\Delta v, x^{\prime \prime}, t\right) / \partial t^{2} \\
& (c / \sqrt{\varepsilon \mu}+(v+\Delta v))^{2} \partial^{2} \Phi^{\prime}\left(v+\Delta v, x^{\prime \prime}, t\right) / \partial x^{\prime \prime 2}=\partial^{2} \Phi^{\prime}\left(v+\Delta v, x^{\prime \prime}, t\right) / \partial t^{2} .
\end{aligned}
$$


For (17.37) the role of equalities (17.32), (17.33) they will play the following transformations of the parameters of the wave:

$$
\begin{gathered}
A^{\prime \prime}(v+\Delta v)=\left(\operatorname{sgn} k_{x}^{\prime}(v)-\frac{\sqrt{\varepsilon \mu} \cdot \Delta v}{c-\sqrt{\varepsilon \mu} \cdot v}\right)^{2} A^{\prime}(v), \omega^{\prime \prime}(v+\Delta v)=\left|\omega^{\prime}(v)-k_{x}^{\prime}(v)\right| \Delta v \\
k_{x}^{\prime \prime}(v+\Delta v)=k_{x}^{\prime}(v) \operatorname{sgn}\left(\omega^{\prime}(v)-k_{x}^{\prime}(v) \Delta v\right) \\
\phi^{\prime \prime}(v+\Delta v)=\phi^{\prime}(v) \operatorname{sgn}\left(\omega^{\prime}(v)-k_{x}^{\prime}(v) \Delta v\right) .
\end{gathered}
$$

For (17.38) the corresponding (17.39), (17.40) conversions of the parameters are determined analogously.

Sequential passage from not prime to prime and is further to the twice prime frame of reference equivalent to direct passage from not prime to twice prime. For example, with $\operatorname{sgn} k_{x}^{\prime}(v)=\operatorname{sgn} k_{x}=1$ from (17.32), (17.39) it is possible to obtain

$$
A^{\prime \prime}(v+\Delta v)=(1-\sqrt{\varepsilon \mu}(v+\Delta v) / c)^{2} A
$$

which is obtained also upon direct transfer to the twice prime frame of reference, since (17.41) it is obtained from (17.32) by replacement $v$ on $v+\Delta v$. In this case the role of equation (17.27) will play the following equation:

$$
\begin{aligned}
& \left(\frac{c}{\sqrt{\varepsilon \mu}}-v\right)^{2} \frac{\partial^{2} \Phi^{\prime}\left(v+\Delta v, x^{\prime \prime}, t\right)}{\partial x^{\prime \prime}}=\frac{\partial^{2} \Phi^{\prime}\left(v, x^{\prime \prime}+\Delta v t, t\right)}{\partial t^{2}}+ \\
& \left(2 \Delta v \frac{\partial^{2}}{\partial t \partial x^{\prime}}+\Delta v^{2} \frac{\partial^{2}}{\partial x^{\prime 2}}\right) \Phi^{\prime}\left(v, x^{\prime \prime}+\Delta v t, t\right)
\end{aligned}
$$

For the derivatives of arbitrary $\mathrm{n}$ - GO of order $\partial^{n} \Phi^{\prime}\left(v+\Delta v, x^{\prime \prime}, t\right) / \partial x^{\prime \prime n}$ $\partial^{n} \Phi^{\prime}\left(v, x^{\prime}, t\right) / \partial x^{\prime n}$ it is possible to use a united designation $\partial^{n} \Phi^{\prime}(v+\Delta v, x, t) / \partial x^{n}$ and $\partial^{n} \Phi^{\prime}(v, x, t) / \partial x^{n} \quad(n=\overline{1, \infty})$, respectively indicating simply derived on the second argument. In accordance with this after substitution (17.35) in (17.42) we will obtain:

$$
\left(\frac{c}{\sqrt{\varepsilon \mu}}-v\right)^{2} \frac{\partial^{2}}{\partial x^{2}}\left(\frac{\Phi^{\prime}(v+\Delta v, x, t)-\Phi^{\prime}(v, x+\Delta v t, t)}{\Delta v}\right)=\left(2 \frac{\partial^{2}}{\partial t \partial x}+\Delta v \frac{\partial^{2}}{\partial x^{2}}\right) \Phi^{\prime}(v, x+\Delta v t, t)
$$

Let us $\Delta v \rightarrow 0$. Let us introduce one additional new derivative, which let us name trans-coordinate and which in the case of the one-dimensional system of space coordinates takes the form:

$$
\frac{\partial^{\prime} \Phi^{\prime}(v, x, t)}{\partial^{\prime} v}=\lim _{\Delta v \rightarrow 0} \frac{\Phi^{\prime}(v+\Delta v, x, t)-\Phi^{\prime}(v, x+\Delta v t, t)}{\Delta v} .
$$

In the determination (17.44) of value $\Phi^{\prime}(v, x+\Delta v t, t), \Phi^{\prime}(v+\Delta v, x, t)$ is described physical field at one and the same point of space, but in the different frame of references (prime and moving relative to it with speed $\Delta v$ twice prime respectively). Within the framework they are equal to the concept of the invariance of field relative to the speed of the motion of observer: 


$$
\Phi^{\prime}(v, x+\Delta v t, t)=\Phi^{\prime}(v+\Delta v, x, t)
$$

the equalities (17.3), (17.45) making identical physical sense, but in connection with to the different pairs of frame of references. However, out of the framework of the indicated concept upon transfer from prime to the twice prime frame of reference the field function at the particular point of space experiences the increase, the limit of relation of which $\Delta v$ with $\Delta v \rightarrow 0$ gives the trans-coordinate derivative (17.44). It is possible to generalize it to the case of the higher orders $(n=\overline{1, \infty})$ :

$$
\begin{gathered}
\frac{\partial^{\prime 1} \Phi^{\prime}(v, x, t)}{\partial^{\prime} v^{1}}=\frac{\partial^{\prime} \Phi^{\prime}(v, x, t)}{\partial^{\prime} v} ; \\
\frac{\partial^{\prime n+1} \Phi^{\prime}(v, x, t)}{\partial^{\prime} v^{n+1}}=\lim _{\Delta v \rightarrow 0} \frac{\frac{\partial^{\prime n} \Phi^{\prime}(v+\Delta v, x, t)}{\partial^{\prime} v^{n}}-\frac{\partial^{\prime n} \Phi^{\prime}(v, x+\Delta v t, t)}{\partial^{\prime} v^{n}}}{\Delta v} .
\end{gathered}
$$

Using trans-coordinate derivatives of the first two orders (17.46), it is possible to represent increase in the field function of in the form corresponding partial summation of series of Taylor:

$$
\Phi^{\prime}(v+\Delta v, x, t)-\Phi^{\prime}(v, x+\Delta v t, t) \approx \frac{\partial^{\prime} \Phi^{\prime}(v, x, t)}{\partial^{\prime} v} \Delta v+\frac{1}{2} \frac{\partial^{\prime 2} \Phi^{\prime}(v, x, t)}{\partial^{\prime} v^{2}} \Delta v^{2} .
$$

Substituting (17.47) in (17.43), equalizing between themselves members with the identical degrees $\Delta v$ in the left and right sides of the received equality, fixing $\Delta v \rightarrow 0$, taking into account that the fact that in this case $\Phi^{\prime}(v, x+\Delta v t, t) \rightarrow \Phi^{\prime}(v, x, t)$ and by adding equality (17.35) in the new form of record (with the use by variable $x$ instead $x^{\prime}$, we will obtain the following system of three equations:

$$
\left\{\begin{array}{l}
\left(\frac{c}{\sqrt{\varepsilon \mu}}-v\right)^{2} \frac{\partial^{2} \Phi^{\prime}(v, x, t)}{\partial x^{2}}=\frac{\partial^{2} \Phi^{\prime}\left(v, x^{\prime}, t\right)}{\partial t^{2}} \\
\left(\frac{c}{\sqrt{\varepsilon \mu}}-v\right)^{2} \frac{\partial \partial^{\prime} \Phi^{\prime}(v, x, t)}{\partial x \partial^{\prime} v}=2 \frac{\partial \Phi^{\prime}\left(v, x^{\prime}, t\right)}{\partial t}, \\
\left(\frac{c}{\sqrt{\varepsilon \mu}}-v\right)^{2} \frac{\partial^{\prime 2} \Phi^{\prime}(v, x, t)}{\partial^{\prime} v^{2}}=2 \Phi^{\prime}\left(v, x^{\prime}, t\right)
\end{array}\right.
$$

System of equations (17.48) can be written down in following that indexed on $\alpha$ the form:

$$
\left(\left(\frac{c}{\sqrt{\varepsilon \mu}}-v\right)^{2} \frac{\partial^{2-\alpha} \partial^{\prime \alpha}}{\partial x^{2-\alpha} \partial^{\prime} v^{\alpha}}-2^{\operatorname{sgn} \alpha} \frac{\partial^{2-\alpha}}{\partial t^{2-\alpha}}\right) \Phi^{\prime}\left(v, x^{\prime}, t\right)=0, \alpha=\overline{0,2}
$$

or in the operator form

$$
\otimes \Phi^{\prime}\left(v, x^{\prime}, t\right)=0,
$$


where $\Leftrightarrow=(\Leftrightarrow \alpha) ; \Leftrightarrow=\left(\left(\frac{c}{\sqrt{\varepsilon \mu}}-v\right)^{\alpha} \frac{\partial^{2-\alpha} \partial^{\prime \alpha}}{\partial x^{2-\alpha} \partial^{\prime} v^{\alpha}}-2^{\operatorname{sgn} \alpha} \frac{\partial^{2-\alpha}}{\partial t^{2-\alpha}}\right)$

- the suitable version of the one-dimensional (case of one axis of space coordinates) differential operator of Dubrovin, which generalizes d'Alembert operator $\square$, who occurs one of his three (zero) components for the laboratory frame of reference, i.e., $\alpha=0, v=0$. Differential equation (17.49) or (17.50) is the gipercontinual one-dimensional homogeneous wave equation, which generalizes, similar to differential equation (17.26) or (17.27), the known one-dimensional homogeneous wave equation (17.28). The vital difference between them (17.26), (17.27) is lies in the fact that the globally trans-coordinate form of gipercontinual wave equation, and (17.49), (17.50) - by its locally trans-coordinate form. Local trans-coordinate means that the equation connects the inertial reference systems, which move relative to each other with the infinitely low speed.

The trans-coordinate of gipercontinual wave equations is ensured by the use in them of the suitable derived field functions. Namely, use by Galileo's derivative reports to equation global trans-coordinate, and by trans-coordinate derivative local.

Thus, is proposed the new approach to the development of the mathematical apparatus for electrodynamics in the direction of the more adequate description of passage from one inertial reference system to another on the basis of gipercontinual ideas about the space and in the time due to the introduction into the examination of the globally and locally trans-coordinate equations, which use new Galilean and trans-coordinate derivatives of field functions, and also the new differential operator of Dubrovin, which generalizes d'Alembert operator. This approach leads to the reformulation of electrodynamics with the passage from the traditional formulation of Hertz- Heaviside to the new trans-coordinate. In this case immediately arise the question about what form they have conversions of electromagnetic field upon transfer from one inertial reference system to another, and will be these conversions the conversions of Mende.

The article is dedicated to answer to this question [58]. Further based on materials the work [58] within the framework of the trans-coordinate formulation of electrodynamics and gipercontinual ideas about the space and the time let us derive the conversions of electromagnetic field upon transfer from one inertial reference system to another.

The convective derivatives of field functions in (17.19), (17.20) can be written down in the form:

$$
\begin{aligned}
& \left(v \mathbf{e}_{1} \cdot \nabla\right) \mathbf{E}\left(\mathbf{r}^{\prime}+t v \mathbf{e}_{1}, t\right)=v\left(\nabla \cdot \mathbf{E}\left(\mathbf{r}^{\prime}+t v \mathbf{e}_{1}, t\right)\right) \mathbf{e}_{1}-\nabla \times\left(v \mathbf{e}_{1} \times \mathbf{E}\left(\mathbf{r}^{\prime}+t v \mathbf{e}_{1}, t\right)\right) ; \\
& \left(v \mathbf{e}_{1} \cdot \nabla\right) \mathbf{B}\left(\mathbf{r}^{\prime}+t v \mathbf{e}_{1}, t\right)=v\left(\nabla \cdot \mathbf{B}\left(\mathbf{r}^{\prime}+t v \mathbf{e}_{1}, t\right)\right) \mathbf{e}_{1}-\nabla \times\left(v \mathbf{e}_{1} \times \mathbf{B}\left(\mathbf{r}^{\prime}+t v \mathbf{e}_{1}, t\right)\right) .
\end{aligned}
$$

We have in view of the first two (17.22) equations taking into account (17.1)(17.2):

$$
\nabla \cdot \mathbf{E}\left(\mathbf{r}^{\prime}+t v \mathbf{e}_{1}, t\right)=0 ; \nabla \cdot \mathbf{B}\left(\mathbf{r}^{\prime}+t v \mathbf{e}_{1}, t\right)=0
$$


After substituting (17.53) in (17.51), (17.52), we will obtain equalities for the convective derivatives:

$$
\begin{aligned}
& \left(v \mathbf{e}_{1} \cdot \nabla\right) \mathbf{E}\left(\mathbf{r}^{\prime}+t v \mathbf{e}_{1}, t\right)=-\nabla \times\left(v \mathbf{e}_{1} \times \mathbf{E}\left(\mathbf{r}^{\prime}+t v \mathbf{e}_{1}, t\right)\right) ; \\
& \left(v \mathbf{e}_{1} \cdot \nabla\right) \mathbf{B}\left(\mathbf{r}^{\prime}+t v \mathbf{e}_{1}, t\right)=-\nabla \times\left(v \mathbf{e}_{1} \times \mathbf{B}\left(\mathbf{r}^{\prime}+t v \mathbf{e}_{1}, t\right)\right) .
\end{aligned}
$$

After substitution (17.54), (17.55) in (17.19), (17.20) we take another form of the Galilean derivatives:

$$
\begin{aligned}
& \frac{\partial^{\prime} \mathbf{E}}{\partial t}\left(v, \mathbf{r}^{\prime}, t\right)=\frac{\partial \mathbf{E}\left(\mathbf{r}^{\prime}+t v \mathbf{e}_{1}, t\right)}{\partial t}-\nabla \times\left(v \mathbf{e}_{1} \times \mathbf{E}\left(\mathbf{r}^{\prime}+t v \mathbf{e}_{1}, t\right)\right) ; \\
& \frac{\partial^{\prime} \mathbf{B}}{\partial t}\left(v, \mathbf{r}^{\prime}, t\right)=\frac{\partial \mathbf{B}\left(\mathbf{r}^{\prime}+t v \mathbf{e}_{1}, t\right)}{\partial t}-\nabla \times\left(v \mathbf{e}_{1} \times \mathbf{B}\left(\mathbf{r}^{\prime}+t v \mathbf{e}_{1}, t\right)\right) .
\end{aligned}
$$

The substitution of Galilean derivatives (17.56), (17.57) into the last two equalities (17.22) gives:

$$
\begin{gathered}
\nabla \times \mathbf{E}^{\prime}\left(v, \mathbf{r}^{\prime}, t\right)=-\partial \mathbf{B}\left(\mathbf{r}^{\prime}+t v \mathbf{e}_{1}, t\right) / \partial t+\nabla \times\left(v \mathbf{e}_{1} \times \mathbf{B}\left(\mathbf{r}^{\prime}+t v \mathbf{e}_{1}, t\right)\right) ; \\
\nabla \times \mathbf{B}^{\prime}\left(v, \mathbf{r}^{\prime}, t\right)=\left(\varepsilon \mu / c^{2}\right)\left(\partial \mathbf{E}\left(\mathbf{r}^{\prime}+t v \mathbf{e}_{1}, t\right) / \partial t-\nabla \times\left(v \mathbf{e}_{1} \times \mathbf{E}\left(\mathbf{r}^{\prime}+t v \mathbf{e}_{1}, t\right)\right)\right) .
\end{gathered}
$$

After substituting last two equations (17.23) in (17.58)-(17.59), we will obtain:

$$
\begin{gathered}
\nabla \times \mathbf{E}^{\prime}\left(v, \mathbf{r}^{\prime}, t\right)=\nabla \times \mathbf{E}\left(\mathbf{r}^{\prime}+t v \mathbf{e}_{1}, t\right)+\nabla \times\left(v \mathbf{e}_{1} \times \mathbf{B}\left(\mathbf{r}^{\prime}+t v \mathbf{e}_{1}, t\right)\right) \\
\nabla \times \mathbf{B}^{\prime}\left(v, \mathbf{r}^{\prime}, t\right)=\nabla \times \mathbf{B}\left(\mathbf{r}^{\prime}+t v \mathbf{e}_{1}, t\right)-\left(\varepsilon \mu / c^{2}\right) \nabla \times\left(v \mathbf{e}_{1} \times \mathbf{E}\left(\mathbf{r}^{\prime}+t v \mathbf{e}_{1}, t\right)\right) .
\end{gathered}
$$

Let us omit the operation of rotor both parts of the equalities (17.60), (17.61):

$$
\begin{gathered}
\mathbf{E}^{\prime}\left(v, \mathbf{r}^{\prime}, t\right)=\mathbf{E}\left(\mathbf{r}^{\prime}+t v \mathbf{e}_{1}, t\right)+v \mathbf{e}_{1} \times \mathbf{B}\left(\mathbf{r}^{\prime}+t v \mathbf{e}_{1}, t\right) ; \\
\mathbf{B}^{\prime}\left(v, \mathbf{r}^{\prime}, t\right)=\mathbf{B}\left(\mathbf{r}^{\prime}+t v \mathbf{e}_{1}, t\right)-\left(\varepsilon \mu / c^{2}\right)\left(v \mathbf{e}_{1} \times \mathbf{E}\left(\mathbf{r}^{\prime}+t v \mathbf{e}_{1}, t\right)\right)
\end{gathered}
$$

Besides the prime frame of reference, which moves relative to laboratory with speed $v$ let us introduce also relatively mobile frame of reference - twice prime, that moves in the same direction with another speed $v+\Delta v$ relative to laboratory. Thus, the twice prime frame of reference moves with relatively prime with speed $\Delta v$, the so that prime frame of reference can be accepted for the new laboratory (relatively fixed), and twice prime - for the new substantive.

Equalities (17.62), (17.63) for them let us write down taking into account the replacement of radius-vector $\mathbf{r}^{\prime}$ on $\mathbf{r}^{\prime \prime}$ :

$$
\begin{gathered}
\mathbf{E}^{\prime}\left(v+\Delta v, \mathbf{r}^{\prime \prime}, t\right)=\mathbf{E}^{\prime}\left(v, \mathbf{r}^{\prime \prime}+t \Delta v \mathbf{e}_{1}, t\right)+\Delta v \mathbf{e}_{1} \times \mathbf{B}^{\prime}\left(v, \mathbf{r}^{\prime \prime}+t \Delta v \mathbf{e}_{1}, t\right) \\
\mathbf{B}^{\prime}\left(v+\Delta v, \mathbf{r}^{\prime \prime}, t\right)=\mathbf{B}^{\prime}\left(v, \mathbf{r}^{\prime \prime}+t \Delta v \mathbf{e}_{1}, t\right)-\left(\varepsilon \mu / c^{2}\right) \Delta v \mathbf{e}_{1} \times \mathbf{E}^{\prime}\left(v, \mathbf{r}^{\prime \prime}+t \Delta v \mathbf{e}_{1}, t\right),
\end{gathered}
$$

Let us write down equalities (17.64), (17.65) in the following form:

$$
\begin{gathered}
\frac{\mathbf{E}^{\prime}\left(v+\Delta v, \mathbf{r}^{\prime \prime}, t\right)-\mathbf{E}^{\prime}\left(v, \mathbf{r}^{\prime \prime}+t \Delta v \mathbf{e}_{1}, t\right)}{\Delta v}=\mathbf{e}_{1} \times \mathbf{B}^{\prime}\left(v, \mathbf{r}^{\prime \prime}+t \Delta v \mathbf{e}_{1}, t\right) \\
\frac{\mathbf{B}^{\prime}\left(v+\Delta v, \mathbf{r}^{\prime \prime}, t\right)-\mathbf{B}^{\prime}\left(v, \mathbf{r}^{\prime \prime}+t \Delta v \mathbf{e}_{1}, t\right)}{\Delta v}=-\frac{\varepsilon \mu}{c^{2}} \mathbf{e}_{1} \times \mathbf{E}^{\prime}\left(v, \mathbf{r}^{\prime \prime}+t \Delta v \mathbf{e}_{1}, t\right) .
\end{gathered}
$$

In (17.66), (17.67) the values $\quad \mathbf{E}^{\prime}\left(v, \mathbf{r}^{\prime \prime}+t \Delta v \mathbf{e}_{1}, t\right), \quad \mathbf{B}^{\prime}\left(v, \mathbf{r}^{\prime \prime}+t \Delta v \mathbf{e}_{1}, t\right)$, $\mathbf{E}^{\prime}\left(v+\Delta v, \mathbf{r}^{\prime \prime}, t\right), \mathbf{B}^{\prime}\left(v+\Delta v, \mathbf{r}^{\prime \prime}, t\right)$ is described the electromagnetic field at one and the same point of space (medium), but in the different frame of references (prime 
and by twice prime). Within the framework they are equal to the concept of the invariance of field relative to the speed of the motion of observer:

$$
\mathbf{E}^{\prime}\left(v, \mathbf{r}^{\prime \prime}+t \Delta v \mathbf{e}_{1}, t\right)=\mathbf{E}^{\prime}\left(v+\Delta v, \mathbf{r}^{\prime \prime}, t\right) ; \mathbf{B}^{\prime}\left(v, \mathbf{r}^{\prime \prime}+t \Delta v \mathbf{e}_{1}, t\right)=\mathbf{B}^{\prime}\left(v+\Delta v, \mathbf{r}^{\prime \prime}, t\right),
$$

The equalities (17.9), (17.68) making identical physical sense, but in connection with to the different pairs of frame of references. However, out of the framework of the indicated concept upon transfer from prime to the twice prime frame of reference the field function at the particular point of space experiences the increase, the limit of relation of which $\mathrm{k} \Delta v$ with $\Delta v \rightarrow 0$ gives that for the first time introduced into 7 the trans-coordinate derivative of the field function:

$$
\begin{aligned}
& \frac{\partial^{\prime} \mathbf{E}^{\prime}\left(v, \mathbf{r}^{\prime \prime}, t\right)}{\partial^{\prime} v}=\lim _{\Delta v \rightarrow 0} \frac{\mathbf{E}^{\prime}\left(v+\Delta v, \mathbf{r}^{\prime \prime}, t\right)-\mathbf{E}^{\prime}\left(v, \mathbf{r}^{\prime \prime}+t \Delta v \mathbf{e}_{1}, t\right)}{\Delta v} ; \\
& \frac{\partial^{\prime} \mathbf{B}^{\prime}\left(v, \mathbf{r}^{\prime \prime}, t\right)}{\partial^{\prime} v}=\lim _{\Delta v \rightarrow 0} \frac{\mathbf{B}^{\prime}\left(v+\Delta v, \mathbf{r}^{\prime \prime}, t\right)-\mathbf{B}^{\prime}\left(v, \mathbf{r}^{\prime \prime}+t \Delta v \mathbf{e}_{1}, t\right)}{\Delta v} .
\end{aligned}
$$

Equalities (17.66), (17.67) with $\Delta v \rightarrow 0$ taking into account (17.69), (17.70) after replacement $\mathbf{r}^{\prime \prime}$ on $\mathbf{r}$ take the form:

$$
\frac{\partial^{\prime} \mathbf{E}^{\prime}\left(v, \mathbf{r}^{\prime}, t\right)}{\partial^{\prime} v}=\mathbf{e}_{1} \times \mathbf{B}^{\prime}\left(v, \mathbf{r}^{\prime}, t\right) ; \frac{\partial^{\prime} \mathbf{B}^{\prime}\left(v, \mathbf{r}^{\prime}, t\right)}{\partial^{\prime} v}=-\frac{\varepsilon \mu}{c^{2}} \mathbf{e}_{1} \times \mathbf{E}^{\prime}\left(v, \mathbf{r}^{\prime}, t\right) .
$$

If equations (17.22) are the globally trans-coordinate differential equations of electrodynamics for the case of isotropic homogeneous medium without the dispersion in the absence of free charges and currents, then equations (17.71) are the locally trans-coordinate differential equations of electrodynamics for the same case. The locality of trans-coordinate, ensured by use by trans-coordinate derivative, means that the connected by differential equations inertial reference systems (conditionally speaking, prime and twice prime) they move relative to each other with the infinitely low speed $\Delta v$. Equations (17.71) form the system, by solving which, it is possible to obtain the conversions of electromagnetic field upon transfer of one inertial reference system into another.

Let us use system of equations (17.71) for obtaining the conversions of electromagnetic field upon transfer from the laboratory frame of reference to the substantive.

Lowering the arguments of functions, let us write down vector products in (17.71) in the form:

$$
\begin{aligned}
& \mathbf{e}_{1} \times \mathbf{B}^{\prime}=\mathbf{e}_{1} \times\left(B^{\prime 1} \mathbf{e}_{1}+B^{\prime 2} \mathbf{e}_{2}+B^{\prime 3} \mathbf{e}_{3}\right)=B^{\prime 2} \mathbf{e}_{3}-B^{\prime 3} \mathbf{e}_{2} ; \\
& \mathbf{e}_{1} \times \mathbf{E}^{\prime}=\mathbf{e}_{1} \times\left(E^{\prime 1} \mathbf{e}_{1}+E^{\prime 2} \mathbf{e}_{2}+E^{\prime 3} \mathbf{e}_{3}\right)=E^{\prime 2} \mathbf{e}_{3}-E^{\prime 3} \mathbf{e}_{2} .
\end{aligned}
$$

Taking into account (17.72), (17.73) the system of equations (17.71) is divided off into two independent systems of two equations each and two additional independent equations: 


$$
\left\{\begin{array} { l } 
{ \frac { \partial ^ { \prime } E ^ { \prime 2 } } { \partial ^ { \prime } v } = - B ^ { \prime 3 } , } \\
{ \frac { \partial ^ { \prime } B ^ { \prime 3 } } { \partial ^ { \prime } v } = - \frac { \varepsilon \mu } { c ^ { 2 } } E ^ { \prime 2 } ; }
\end{array} \left\{\begin{array}{l}
\frac{\partial^{\prime} E^{\prime 3}}{\partial^{\prime} v}=B^{\prime 2}, \quad \\
\frac{\partial^{\prime} B^{\prime 2}}{\partial^{\prime} v}=\frac{\varepsilon \mu}{c^{2}} E^{\prime 3} ;
\end{array} \quad \frac{\partial^{\prime} E^{\prime 1}}{\partial^{\prime} v}=0 ; \frac{\partial^{\prime} B^{\prime 1}}{\partial^{\prime} v}=0 .\right.\right.
$$

We differentiate the first equations of systems (17.74) and will substitute them into the second equations:

$$
\frac{\partial^{\prime 2} E^{\prime 2}}{\partial^{\prime} v^{2}}=\frac{\varepsilon \mu}{c^{2}} E^{\prime 2} ; \frac{\partial^{\prime 2} E^{\prime 3}}{\partial^{\prime} v^{2}}=\frac{\varepsilon \mu}{c^{2}} E^{\prime 3} ; \frac{\partial^{\prime 2} B^{\prime 2}}{\partial^{\prime} v^{2}}=\frac{\varepsilon \mu}{c^{2}} B^{\prime 2} ; \frac{\partial^{\prime 2} B^{\prime 3}}{\partial^{\prime} v^{2}}=\frac{\varepsilon \mu}{c^{2}} B^{\prime 3} .
$$

The general solution of equations (17.75) is expressed as the arbitrary constants $C_{1}, \ldots, C_{10}$ :

$$
E^{\prime 1}=C_{1} ; E^{\prime 2}=C_{2} \cosh \frac{\sqrt{\varepsilon \mu} \nu}{c}+C_{3} \sinh \frac{\sqrt{\varepsilon \mu} \nu}{c} ; E^{\prime 3}=C_{4} \cosh \frac{\sqrt{\varepsilon \mu} \nu}{c}+C_{5} \sinh \frac{\sqrt{\varepsilon \mu} \nu}{c},
$$

$$
B^{\prime 1}=C_{6} ; B^{\prime 2}=C_{7} \cosh \frac{\sqrt{\varepsilon \mu} \nu}{c}+C_{8} \sinh \frac{\sqrt{\varepsilon \mu} v}{c} ; B^{\prime 3}=C_{9} \cosh \frac{\sqrt{\varepsilon \mu \nu}}{c}+C_{10} \sinh \frac{\sqrt{\varepsilon \mu \nu}}{c}
$$

Since we search for the conversions of electromagnetic field upon transfer from the laboratory frame of reference, then the desired particular solutions of equations (17.75) must with $v=0$ describe electromagnetic field in the laboratory frame of reference, i.e., satisfy equalities (17.8) and (17.74), and the, which means, following totality of the equalities:

$$
\begin{gathered}
E^{\prime 1}\left(0, \mathbf{r}^{\prime}, t\right)=E^{1}\left(\mathbf{r}^{\prime}, t\right) ; E^{\prime 2}\left(0, \mathbf{r}^{\prime}, t\right)=E^{2}\left(\mathbf{r}^{\prime}, t\right) ; E^{\prime 3}\left(0, \mathbf{r}^{\prime}, t\right)=E^{3}\left(\mathbf{r}^{\prime}, t\right) ; \\
B^{\prime 1}\left(0, \mathbf{r}^{\prime}, t\right)=B^{1}\left(\mathbf{r}^{\prime}, t\right) ; B^{\prime 2}\left(0, \mathbf{r}^{\prime}, t\right)=B^{2}\left(\mathbf{r}^{\prime}, t\right) ; B^{\prime 3}\left(0, \mathbf{r}^{\prime}, t\right)=B^{3}\left(\mathbf{r}^{\prime}, t\right) ; \\
\frac{\partial^{\prime} E^{\prime 2}\left(0, \mathbf{r}^{\prime}, t\right)}{\partial^{\prime} v}=-B^{3}\left(\mathbf{r}^{\prime}, t\right) ; \frac{\partial^{\prime} E^{\prime 3}\left(0, \mathbf{r}^{\prime}, t\right)}{\partial^{\prime} v}=B^{2}\left(\mathbf{r}^{\prime}, t\right) ; \\
\frac{\partial^{\prime} B^{\prime 2}\left(0, \mathbf{r}^{\prime}, t\right)}{\partial^{\prime} v}=\frac{\varepsilon \mu}{c^{2}} E^{3}\left(\mathbf{r}^{\prime}, t\right) ; \frac{\partial^{\prime} B^{\prime 3}\left(0, \mathbf{r}^{\prime}, t\right)}{\partial^{\prime} v}=-\frac{\varepsilon \mu}{c^{2}} E^{2}\left(\mathbf{r}^{\prime}, t\right) .
\end{gathered}
$$

By substitution (17.76), (17.77) in (17.78)-(17.81) let us find the values of constants $C_{1}, \ldots, C_{10}$, as a result what after the substitution of these constants in (17.76), (17.77) we will obtain the resultant expression in the component form for the desired conversions of electromagnetic field upon transfer from the laboratory frame of reference to the substantive:

$$
E^{\prime 1}\left(v, \mathbf{r}^{\prime}, t\right)=E^{1}\left(\mathbf{r}^{\prime}, t\right) ; B^{\prime 1}\left(v, \mathbf{r}^{\prime}, t\right)=B^{1}\left(\mathbf{r}^{\prime}, t\right)
$$




$$
\begin{aligned}
& E^{\prime 2}\left(v, \mathbf{r}^{\prime}, t\right)=E^{2}\left(\mathbf{r}^{\prime}, t\right) \cosh \frac{\sqrt{\varepsilon \mu} v}{c}-\frac{c}{\sqrt{\varepsilon \mu}} B^{3}\left(\mathbf{r}^{\prime}, t\right) \sinh \frac{\sqrt{\varepsilon \mu \nu}}{c} \\
& E^{\prime 3}\left(v, \mathbf{r}^{\prime}, t\right)=E^{3}\left(\mathbf{r}^{\prime}, t\right) \cosh \frac{\sqrt{\varepsilon \mu} v}{c}+\frac{c}{\sqrt{\varepsilon \mu}} B^{2}\left(\mathbf{r}^{\prime}, t\right) \sinh \frac{\sqrt{\varepsilon \mu \nu}}{c} \\
& B^{\prime 2}\left(v, \mathbf{r}^{\prime}, t\right)=B^{2}\left(\mathbf{r}^{\prime}, t\right) \cosh \frac{\sqrt{\varepsilon \mu} v}{c}+\frac{\sqrt{\varepsilon \mu}}{c} E^{3}\left(\mathbf{r}^{\prime}, t\right) \sinh \frac{\sqrt{\varepsilon \mu \nu}}{c} \\
& B^{\prime 3}\left(v, \mathbf{r}^{\prime}, t\right)=B^{3}\left(\mathbf{r}^{\prime}, t\right) \cosh \frac{\sqrt{\varepsilon \mu \nu}}{c}-\frac{\sqrt{\varepsilon \mu}}{c} E^{2}\left(\mathbf{r}^{\prime}, t\right) \sinh \frac{\sqrt{\varepsilon \mu \nu}}{c}
\end{aligned}
$$

In the vector form the same conversions take the following form:

$$
\begin{gathered}
\mathbf{E}^{\prime}\left(v, \mathbf{r}^{\prime}, t\right)=\mathbf{E}\left(\mathbf{r}^{\prime}, t\right) \cosh \frac{\sqrt{\varepsilon \mu} \nu}{c}+\frac{c}{\sqrt{\varepsilon \mu}} \mathbf{e}_{1} \times \mathbf{B}\left(\mathbf{r}^{\prime}, t\right) \sinh \frac{\sqrt{\varepsilon \mu} v}{c} \\
\mathbf{B}^{\prime}\left(v, \mathbf{r}^{\prime}, t\right)=\mathbf{B}\left(\mathbf{r}^{\prime}, t\right) \cosh \frac{\sqrt{\varepsilon \mu} v}{c}-\frac{\sqrt{\varepsilon \mu}}{c} \mathbf{e}_{1} \times \mathbf{E}\left(\mathbf{r}^{\prime}, t\right) \sinh \frac{\sqrt{\varepsilon \mu \nu}}{c} .
\end{gathered}
$$

It is easy to see that the conversions (17.82)-(17.88) are known conversions of Mende [58].

Thus, the conversions of Mende obtain a sufficient theoretical substantiation within the framework of the trans-coordinate formulation of electrodynamics, connected with the gipercontinual ideas about the space and the time, and also with the concept not of the invariance of electric charge relative to the speed of the motion of observer. Together with that been in [50] direct experimental confirmation of the concept not of the invariance of electric charge, this is convincing evidence of their larger adequacy of physical reality on the comparison not only with the classical, but also with the relativistic conversions of electromagnetic field, or the convincing evidence of the justification of the transfer of electrodynamics from the traditional formulation of Hertz- Heaviside to the trans-coordinate. The sequential development of trans-coordinate electrodynamics is capable of not only deriving on the new qualitative level of idea about the space and the time, but also of opening the fundamentally new horizons of the development engineering and technologies due to the discovery and the mastery of new physical phenomena and effects.

\section{$\S 18$. Electric fields in the concept of the scalar-vector potential}

In the concept of scalar-vector potential it is possible to isolate, at least, three cases of the dependence of the tension of electric field on the charge rates, which characterize the different versions of this concept. 


\section{1) Nonpotential electric field}

If speech goes about the electric field of the single charge $e$, then its electric field will be determined by the relationship:

$$
\mathbf{E}=\frac{e}{4 \pi \varepsilon_{0} \mathbf{r}^{2}}\left(\mathbf{e}_{v}+\operatorname{ch} \frac{\sqrt{\mathbf{v}^{2}}}{c} \mathbf{e}_{\perp}\right)
$$

where $\mathbf{e}_{v}=\frac{\mathbf{r} \cdot \mathbf{v}}{\mathbf{v}^{2} \sqrt{\mathbf{r}^{2}}} \mathbf{v}-$ the projection on the direction of the vector $\mathbf{v}$ of the unit vector $\mathbf{e}_{r}=\mathbf{r} / \sqrt{\mathbf{r}^{2}}$, of collinear to the vector $\mathbf{r} ; \mathbf{e}_{\perp}=\mathbf{e}_{r}-\mathbf{e}_{v}=\frac{\mathbf{r}}{\sqrt{\mathbf{r}^{2}}}-\frac{\mathbf{r} \cdot \mathbf{v}}{\mathbf{v}^{2} \sqrt{\mathbf{r}^{2}}} \mathbf{v}-$ the projection of the unit vector $\mathbf{e}_{r}=\mathbf{r} / \sqrt{\mathbf{r}^{2}}$, of collinear to vector $\mathbf{r}$, on the direction of normal to the vector $\mathbf{v}$, of that lying at one plane $\mathbf{s} \mathbf{r}$.

Let us fix certain moment of time. Let us select the system of rectangular Cartesian space coordinates $O X Y Z$ so that at this moment the time the origin of coordinates would coincide with the moving point charge $e$, axis $O X$ would be directed posigrade of charge $\mathbf{v}$, and vector $\mathbf{r}$ it would lie in the plane $X O Y$. Then vector $\mathbf{r}$ can be assigned by two coordinates $x$, also, $y$ along the axes $O X$ and $O Y$ respectively. In this case longitudinal (along the axis $O X$ ) and transverse (along the axis $O Y$ ) of the component of the vector of the tension of electric field at point $\mathbf{r}$ they will be equal respectively

$$
E_{x}=\frac{e}{4 \pi \varepsilon_{0}} \cdot \frac{x}{\sqrt{\left(x^{2}+y^{2}\right)^{3}}} ; E_{y}=\frac{e}{4 \pi \varepsilon_{0}} \operatorname{ch} \frac{\sqrt{\mathbf{v}^{2}}}{c} \cdot \frac{y}{\sqrt{\left(x^{2}+y^{2}\right)^{3}}} .
$$

Integration of these components for the appropriate coordinates gives:

$$
\int E_{x} d x=-\frac{e}{4 \pi \varepsilon_{0}} \cdot \frac{1}{\sqrt{x^{2}+y^{2}}}+C_{1}(y) ; \int E_{y} d y=-\frac{e}{4 \pi \varepsilon_{0}} \operatorname{ch} \frac{\sqrt{\mathbf{v}^{2}}}{c} \cdot \frac{1}{\sqrt{x^{2}+y^{2}}}+C_{2}(x),
$$

where $C_{1}(y)$ and $C_{2}(x)$-integration constant.

With $\sqrt{\mathbf{v}^{2}}=0$ the electric field proves to be potential with the known potential

$$
\varphi(x, y)=\frac{e}{4 \pi \varepsilon_{0}} \cdot \frac{1}{\sqrt{x^{2}+y^{2}}},
$$

but with $\sqrt{\mathbf{v}^{2}} \neq 0$ the potentiality of field it is disrupted, i.e., there does not exist this function of potential, through which it would be possible to express the field strength according to the formula

$$
\mathbf{E}=-\nabla \varphi .
$$

Therefore such electric field of the moving charge must be examined as the superposition

$$
\mathbf{E}=\mathbf{E}_{v}+\mathbf{E}_{\perp}
$$

longitudinal electric field $\mathbf{E}_{v}$ with the potential (let us name its longitudinal potential) 


$$
\varphi_{v}(x, y)=\frac{e}{4 \pi \varepsilon_{0}} \cdot \frac{1}{\sqrt{x^{2}+y^{2}}}
$$

and transverse electric field $\mathbf{E}_{\perp}$ with the potential (let us name its transverse potential)

$$
\varphi_{\perp}(x, y)=\frac{e}{4 \pi \varepsilon_{0}} \operatorname{ch} \frac{\sqrt{\mathbf{v}^{2}}}{c} \cdot \frac{1}{\sqrt{x^{2}+y^{2}}},
$$

the fields $\mathbf{E}_{v} \quad \mathbf{E}_{\perp}$ having nontrivial components only on $O X$ and $O Y$ :

$$
\mathbf{E}_{v}=\frac{\partial \varphi_{v}}{\partial x} ; \mathbf{E}_{\perp}=\frac{\partial \varphi_{\perp}}{\partial y} .
$$

Therefore in this case it is more correct to speak not about the concept of the scalar potential of charge, which depends on the speed, but about the concept of invariant longitudinal scalar potential and transverse scalar potential depending on the speed.

\section{2) Potential electric field of Mende}

Let us fix certain moment of time. Let us select the system of rectangular Cartesian space coordinates $O X Y Z$ so that at this moment the time the origin of coordinates would coincide with the moving point charge $e$, axis $O Y$ would be directed posigrade of charge $\mathbf{v}$, and vector $\mathbf{r}$, directed from one point of the concentration of charge to the next, at which the field is determined, it lay in the plane $X O Y$. Then vector $\mathbf{r}$ can be assigned by two coordinates $x$, also, $y$ along the axes $O X$ and $O Y$ respectively.

Let us assume that electric field is the potential field, given by the scalar potential

$$
\varphi(\mathbf{r})=e \operatorname{ch}\left(v_{\perp}(\mathbf{r}) / c\right) /\left(4 \pi \varepsilon_{0} r\right)
$$

where $\mathbf{r}=(x, y) \quad$ the radius-vector of the point, at which is determined the field; $r=\sqrt{\mathbf{r}^{2}}=\sqrt{x^{2}+y^{2}} \quad$ - the distance between the charge and the point, at which is determined the field; $v_{\perp}(\mathbf{r})$ - the component of charge rate $e$, is normal $\mathrm{k} \mathbf{r}$.

Let there be $x>0, y>0$, then:

$$
v_{\perp}(x, y)=\frac{v x}{\sqrt{x^{2}+y^{2}}}=\frac{v x}{r} ; \varphi(x, y)=\frac{e}{4 \pi \varepsilon_{0} \sqrt{x^{2}+y^{2}}} \operatorname{ch} \frac{v x}{c \sqrt{x^{2}+y^{2}}}=\frac{e}{4 \pi \varepsilon_{0} r} \operatorname{ch} \frac{v x}{c r} \text {. }
$$

The strength of this field has transverse component $E_{x}(x, y)$ and longitudinal component $E_{y}(x, y)$, which they are determined from the formulas:

$$
\begin{gathered}
E_{x}(x, y)=-\frac{\partial \varphi(x, y)}{\partial x}=\frac{e}{4 \pi \varepsilon_{0} r^{3}}\left(x \operatorname{ch} \frac{v x}{c r}-\frac{v y^{2}}{c r} \operatorname{sh} \frac{v x}{c r}\right) \\
E_{y}(x, y)=-\frac{\partial \varphi(x, y)}{\partial y}=\frac{e y}{4 \pi \varepsilon_{0} r^{3}}\left(\operatorname{ch} \frac{v x}{c r}-\frac{v x}{c r} \operatorname{sh} \frac{v x}{c r}\right) . \\
-115-
\end{gathered}
$$




\section{3) Potential electric field with the transversely deformed scalar potential}

Let us select the system of rectangular Cartesian space coordinates in the manner that in the case 2 . The electrostatic potential of charge takes the form:

$$
\varphi(x, y)=e /\left(4 \pi \varepsilon_{0} r\right)=e /\left(4 \pi \varepsilon_{0} \sqrt{x^{2}+y^{2}}\right),
$$

where $\mathbf{r}=(x, y) \quad$ - the radius-vector of the point, at which is determined the field; $r=\sqrt{\mathbf{r}^{2}}=\sqrt{x^{2}+y^{2}}$ - the distance between the charge and this point.

Let us assume that the dynamic potential of charge is the transversely deformed electrostatic potential. Then we obtain the potential electric field, described by the scalar potential,

$$
\varphi_{\perp}(v, x, y)=\varphi(x / \operatorname{ch}(v / c), y),
$$

where $v=\sqrt{\mathbf{v}^{2}}$ - the module of charge rate, moreover

$$
\varphi_{\perp}(0, x, y)=\varphi(x, y) \text {. }
$$

Taking into account expression for the electrostatic potential, we obtain formula for the dynamic potential:

$$
\varphi_{\perp}(v, x, y)=\frac{e \operatorname{ch}(v / c)}{4 \pi \varepsilon_{0} \sqrt{x^{2}+y^{2} \operatorname{ch}^{2}(v / c)}} .
$$

The transverse $E_{\perp x}(v, x, y)$ and longitudinal $E_{\perp y}(v, x, y)$ components of the field strength will be written down in the form

$$
\begin{aligned}
& E_{\perp x}(v, x, y)=-\frac{\partial \varphi_{\perp}(v, x, y)}{\partial x}=\frac{e x \operatorname{ch}(v / c)}{4 \pi \varepsilon_{0} \sqrt{\left(x^{2}+y^{2} \operatorname{ch}^{2}(v / c)\right)^{3}}} ; \\
& E_{\perp y}(v, x, y)=-\frac{\partial \varphi_{\perp}(v, x, y)}{\partial y}=\frac{e y \operatorname{ch}^{3}(v / c)}{4 \pi \varepsilon_{0} \sqrt{\left(x^{2}+y^{2} \operatorname{ch}^{2}(v / c)\right)^{3}}} .
\end{aligned}
$$

With $x \neq 0, y=0$ we have:

$$
E_{\perp x}(v, x, y)=\frac{e \operatorname{ch}(v / c)}{4 \pi \varepsilon_{0} x^{2}} ; E_{\perp y}(v, x, y)=0 .
$$

With $x=0, y \neq 0$ we have:

$$
E_{\perp x}(v, x, y)=0 ; E_{\perp y}(v, x, y)=\frac{e}{4 \pi \varepsilon_{0} y^{2}} .
$$

\section{Conclusions of three cases examined are the following}

Thus, three versions of the determination of electrical pour on in the concept of the scalar- vector potential proposed:

1) the nonpotential field, whose longitudinal component of tension does not depend on speed, but transverse - depends; 
2) potential field in Mende's version;

3) potential field with the transversely deformed scalar potential.

Last two versions characterize the potential field, in which in general case both components depend on speed; however, for this field it is possible to isolate two extreme special cases for the points, at which this field is determined: if to point (at the coordinate system proposed these points lie on the axis the transverse component $O Y$ ) of field it is absent into some, then longitudinal does not depend on speed; if to point (at the coordinate system proposed these points lie on the axis the longitudinal component $O X$ ) of field it is absent into some, then transverse depends on speed by the simple dependence, which is obtained by the multiplication of the corresponding strength of electrostatic field to the coefficient $\operatorname{ch}(v / c)$.

The potentiality of electric field means that during the motion in this field of trial charge along the locked trajectory the work is not accomplished. But if scalar potential changes in the time, then in the time changes electric field the, as a result of which this property of the absence of the perfect work, strictly speaking, it is not carried out. However, in this case for each assigned moment of time potential electric field (last two versions) can be defined as the undertaken with the opposite sign gradient of scalar potential.

An important difference in the first and third versions in the first version lies in the fact that occurs the lateral deformation of the strength of the field (it it leads to the nonpotentiality of field), and in the third - the lateral deformation the scalar potential field (it leads to the dependence on the speed in general case of both components of the field strength).

In the present monograph the basis is undertaken the second version of concept, although the third version from a physical point of view is presented to us not less, but, possibly, by more promising, and the first version is also is sufficiently promising. In connection with this, one of the most important tasks of further studies - the determination of version, most adequate of reality.

\section{Laws of the electro-electrical induction}

Ampere law in the vector form known long before the appearance of Maxwell's equations determines magnetic field at the particular point:

$$
\mathbf{H}=\frac{1}{4 \pi} \int \frac{I d \mathbf{l} \times \mathbf{r}}{r^{3}},
$$

where $I$ - current in the element $d \mathbf{l}, \mathbf{r}$ - vector, directed from to the point $d \mathbf{l}$. It is possible to show that

$$
\frac{d \mathbf{l} \times \mathbf{r}}{r^{3}}=\operatorname{grad}\left(\frac{1}{r}\right) \times d \mathbf{l}=\operatorname{rot}\left(\frac{d \mathbf{l}}{r}\right)-\frac{1}{r} \operatorname{rot} d \mathbf{l}=\operatorname{rot}\left(\frac{d \mathbf{l}}{r}\right),
$$

therefore finally we will obtain:

$$
\mathbf{H}=\operatorname{rot} \int I\left(\frac{d \mathbf{l}}{4 \pi r}\right)=\operatorname{rot} \mathbf{A}_{\mathbf{H}},
$$

where 


$$
\mathbf{A}_{\mathbf{H}}=\int I\left(\frac{d \mathbf{l}}{4 \pi r}\right)
$$

the remarkable property of this expression is that that the vector potential depends from the distance to the observation point as $1 / r$. Specifically, this property makes it possible to obtain emission laws.

Since. $I=g v$, where $g$ - a charge of the unit of the length of conductor, from (18.1) we have:

$$
\mathbf{A}_{\mathbf{H}}=\int \frac{g v d \mathbf{l}}{4 \pi r} .
$$

for the single charge $e$ this relationship takes the form:

$$
\mathbf{A}_{\mathbf{H}}=\frac{e \mathbf{v}}{4 \pi r} .
$$

Since

$$
\mathbf{E}=-\mu \frac{\partial \mathbf{A}_{\mathbf{H}}}{\partial t},
$$

that in the general case we have (here $a$ - the acceleration of charge):

$$
\mathbf{E}=-\mu \int \frac{g \frac{\partial v}{\partial t} d \mathbf{l}}{4 \pi r}=-\mu \int \frac{g a d \mathbf{l}}{4 \pi r}
$$

and we will obtain for a special case of single charge:

$$
\mathbf{E}=-\frac{\mu e \mathbf{a}}{4 \pi r} .
$$

If we in (18.2), (18.3) consider the propagation time delay $(t-r / c)$ of potentials and that the fact that for the vacuum $\mu=1 /\left(\varepsilon_{0} c^{2}\right)$, these equalities will take the form:

$$
\begin{gathered}
\mathbf{E}=-\mu \int \frac{g a(t-r / c) d \mathbf{l}}{4 \pi r}=-\int \frac{g a(t-r / c) d \mathbf{l}}{4 \pi \varepsilon_{0} c^{2} r}, \\
\mathbf{E}=-\frac{e \mathbf{a}(t-r / c)}{4 \pi \varepsilon_{0} c^{2} r} .
\end{gathered}
$$

Wave equations (18.4), (18.5) are here obtained with the use of concepts of magnetic field and its vector potential, but by themselves they only give the dependence of electric field on its inducing moving charges. The knowledge of this dependence allows with the description of the processes of emission not at all to use the concepts of magnetic field and its vector potential.

Let the charge $e$ vary along the axis $y$ in the environment of point 0 with the amplitude, considerably smaller than the distance $r$ from the observed point (1) with $y=0$ to the charge (Fig. 13). This relationship is wave equation and defines both the amplitude and phase responses of the wave of the electric field, radiated by the moving charge. 


$$
E_{y}(x, t)=-\frac{e}{4 \pi \varepsilon_{0} c^{2} x} \frac{\partial v_{y}(t-x / c)}{\partial t}=-\frac{e a_{y}(t-x / c)}{4 \pi \varepsilon_{0} c^{2} x}
$$

where $v_{y}(t-x / c), \quad a_{y}(t-\not x) c$ - being late speed and the acceleration of charge.

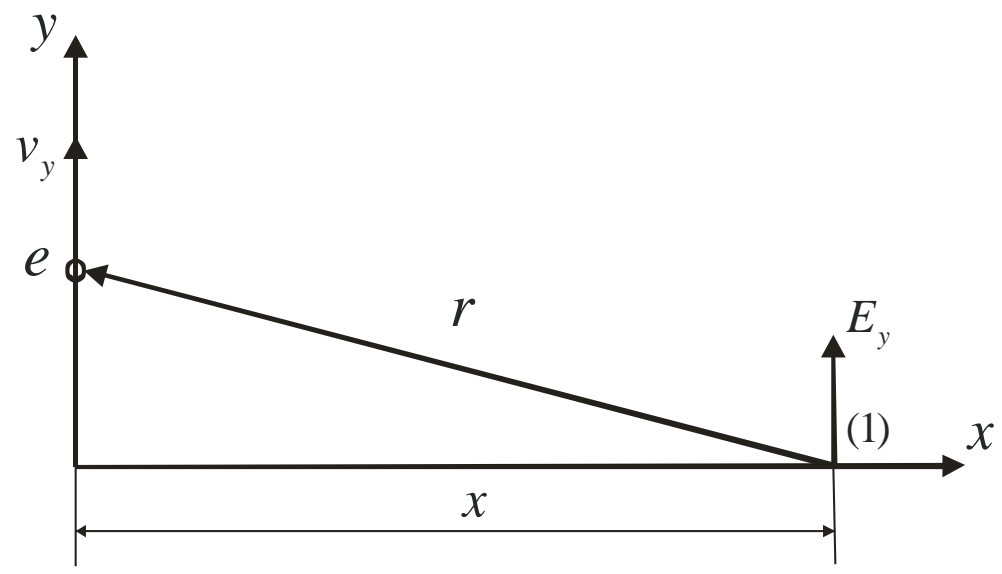

Fig. 13. Diagram of shaping of the induced electric field

After selecting by the direction of emission vector in the plane $x y$, component with the axis $y$ angle $\alpha$, from (18.6) we will obtain the relationship, which determines the known radiation pattern of dipole source (complete diagram it is symmetrical relative to the axis $y$ ):

$$
E_{y}(x, t, \alpha)=-\frac{e a_{y}(t-x / c) \sin \alpha}{4 \pi \varepsilon_{0} c^{2} x}=-\frac{1}{\varepsilon_{0} c^{2}} \frac{\partial A_{H}(t-x / c)}{\partial t}=-\mu_{0} \frac{\partial A_{H}(t-x / c)}{\partial t} .
$$

Known in the electrodynamics being late vector potential

$$
\frac{e v_{y}(t-x / c)}{4 \pi x}=A_{H}(t-x / c)
$$

it is introduced on the basis of the law of electro-electrical induction. The electric fields of wave are gradient, but are not vortex as in Maxwell's equations.

With the aid of (18.7) it is possible to describe the dipole emission of the charges, which are varied in the electric field. Time derivative of the dipole moment (vector $\mathbf{d}$ it is directed from the negative charge toward the positive) $\mathbf{p}=e \mathbf{d}$ is connected with the current:

$$
e \mathbf{v}=e \frac{\partial \mathbf{d}}{\partial t}=\frac{\partial \mathbf{p}}{\partial t}, \mathbf{v}=\frac{1}{e} \frac{\partial \mathbf{p}}{\partial t}, \mathbf{a}=\frac{\partial \mathbf{v}}{\partial t}=\frac{1}{e} \frac{\partial^{2} \mathbf{p}}{\partial t^{2}}
$$

The substitution of last equality for $\mathbf{a}$ in (18.7) gives the known [1] emission law of the being varied dipole

$$
\mathbf{E}=-\frac{1}{4 \pi r \varepsilon_{0} c^{2}} \frac{\partial^{2} \mathbf{p}(t-r / c)}{\partial t^{2}}
$$

Thus, the field of the being varied electric dipole is determined by the superposition of electrical induction pour on the emissions (18.6)-(18.8), of those 
connected with the acceleration of charge, and electrical pour on static dipole, that are changed according to dependence on the time of the distance between the charges. The process of emission is connected with the transformation of energy pour on static dipole into the energy pour on emissions.

Pour on the absence (18.6)-(18.8) in the magnetic and vector potentials confirms the fundamentality of electrical pour on.

Relationship (18.7) makes it possible to obtain the laws of reflection and scattering of electrical pour on by the totality of the charges, set to the forced motion by external (strange) electric field. The superposition of electrical pour on, radiated by all charges, it is electrical wave.

The acceleration of charge under the action of strange electric field $E_{y}^{\prime}=E_{y 0}^{\prime} \sin \omega t$ is equal

$$
a=-\frac{e}{m} E_{y 0}^{\prime} \sin \omega t .
$$

Taking into account this relationship (18.5) assumes the form

$$
E_{y}(x, t, \alpha)=\frac{e^{2} \sin \alpha}{4 \pi \varepsilon_{0} c^{2} m x} E_{y 0}^{\prime} \sin \omega\left(t-\frac{x}{c}\right)=\frac{K}{x} E_{y 0}^{\prime} \sin \omega\left(t-\frac{x}{c}\right)
$$

where the determining ability of charge to re-emit external field the value

$$
K=\frac{e^{2} \sin \alpha}{4 \pi \varepsilon_{0} c^{2} m}
$$

let us name the coefficient of scattering (re-emission) charge in the assigned direction.

The current wave of the displacement accompanies the wave of electric field:

$$
j_{y}(x, t)=\varepsilon_{0} \frac{\partial E_{y}}{\partial t}=-\frac{e \sin \alpha}{4 \pi c^{2} x} \frac{\partial^{2} v_{y}(t-x / c)}{\partial t^{2}} .
$$

If charge accomplishes its motion under the action of the electric field of , then bias current in the distant zone will be written down as

$$
j_{y}(x, t)=-\frac{e^{2} \omega}{4 \pi c^{2} m x} E_{y 0}^{\prime} \cos \omega(t-x / c)
$$

The wave of electric field (18.9) and laggard behind it on $\pi / 2$ the current wave of displacement (18.10) form the wave, for the first time described into [11, 13] that named there electriccurent wave.

In parallel with the electrical waves it is possible to introduce magnetic waves, if we assume that

$$
\begin{gathered}
\mathbf{j}=\varepsilon_{0} \frac{\partial \mathbf{E}}{\partial t}=\operatorname{rot} \mathbf{H}, \\
\operatorname{div} \mathbf{H}=0 .
\end{gathered}
$$

Such magnetic field - is vortex. Comparing (18.9) and (18.10) we obtain:

$$
\frac{\partial H_{z}(x, t)}{\partial x}=\frac{e^{2} \omega \sin \alpha}{4 \pi c^{2} m x} E_{y 0}^{\prime} \cos \omega\left(t-\frac{x}{c}\right) .
$$

Integrating this relationship on the coordinate, we find the value of the magnetic field 


$$
H_{z}(x, t)=\frac{e^{2} \sin \alpha}{4 \pi c m x} E_{y 0}^{\prime} \sin \omega\left(t-\frac{x}{c}\right) .
$$

Thus, relationship (18.9), (18.10) and (18.12) can be named the laws of electrical induction, since they give the direct coupling between the electric fields, applied to the charge, and by fields and by currents induced by this charge in its environment. Charge itself comes in the role of the transformer, which ensures this reradiation. Magnetic field (18.12) is directed normally both toward the electric field and toward the direction of propagation. In this case it is executed:

$$
\frac{E_{y}(x, t)}{H_{z}(x, t)}=\frac{1}{\varepsilon_{0} c}=\sqrt{\frac{\mu_{0}}{\varepsilon_{0}}}=Z,
$$

where $Z$ - wave drag of free space.

Wave drag determines the active power of losses on the single area, located normal to the direction of propagation of the wave:

$$
P=Z E_{y 0}^{2} / 2 \text {. }
$$

This relationship determines the power flux of the electriccurent wave through this area according to Poynting's theorem.

Thus, any wave process in the fields of electromagnetic nature is reduced to the the electriccurent to waves in the space, characterized by its wave drag. This examination does not require the attraction of the concepts of magnetic field and its vector potential. Nevertheless, these concepts can be additionally introduced, clearly dividing fields to the gradient (electrical) and the vortex (magnetic) according to Helmholtz's theorem, which says, that any single-valued and continuous vectorial field $\mathbf{F}$, turning into zero at infinity, can be represented, and besides uniquely, in the form the sum of the gradient of a certain scalar function $\varphi$ and rotor of a certain vector function $\mathbf{C}$, divergence of which is equal to zero:

$$
\mathbf{F}=\operatorname{grad} \varphi+\operatorname{rot} \mathbf{C}, \operatorname{div} \mathbf{C}=0 .
$$

The grit, which are erected around the magnet poles, they indicate the presence of some power pour on potential nature, but in its time from this they did not make proper conclusions, and significant [3]: "underwater part of the iceberg" remained invisible of almost two hundred years.

Thus, the construction of electrodynamics should have been begun from the acknowledgement of the dependence of the electric field of charge on the parameters of its motion, as this many chief scientists of that time proposed. But nature very deeply hides its secrets, and in order to come to this simple conclusion, it was necessary to pass way by length almost into two centuries.

Is exponential quotation from 3: but "in what does consist the basic initial reason for the discrepancy of the built by Maxwell electrodynamics? For the single-valued answer to this question... it should be noted that even in its time of amperes, Grossman, Gauss, Lentz, Neumann, Veber, Riemann and other they stood on the point of view, that, without being turned to the concept "of magnetic field", any magnetic interactions can be reduced to usual interactions of current elements or moving charges... in the electrodynamics repossessed then the point of view of Faraday and Maxwell, that the electrical and "magnetic" fields are the independent physical essences, although connected together. In the pre- 
vailing then historical situation given, erroneous from a physical point of view, assumptions predetermined by themselves entire further motion of the development of electrodynamics with the deliberately placed into it insoluble contradictions and the paradoxes". And further there: "for the noncontradictory reflection of the physical essence of the laws of electromagnetism necessary to completely forego any concepts "magnetic field" as certain independent physical essence... for determining the forces of interaction of moving in the physical vacuum of real space electric charges completely sufficient to consider the deformation of electrical pour on these charges, caused by the trivial effects of the being late potentials... To there remains only be surprised at the sagacity of the ampere, which warned that if we in the electrodynamics do not forego ourselves the concept "magnet", then subsequently this threatens by incredible confusion in the theory".

This is how electrodynamics during entire its history little moved. The phenomenon of electromagnetic induction Faraday opened in 1831, and already almost 200 years his study remains practically constant, but the physical causes for many electrodynamic phenomena - not understood. Certainly, for his time Faraday was genius, but that they did make physics after it? Even brilliant Maxwell and Hertz did not establish the fundamental role of the dependence of the electric field of charge on the parameters of his motion. Subsequently such scientists as Nikolayev G.V. and Marinov S. their theoretical and experimental studies were conducted in this direction, but proper acknowledgement as scientific association these works so did not obtain.

After entire aforesaid it is possible to remove construction forests, such as magnetic field and magnetic vector potential, which do not allow here already almost two hundred years to see the building of electrodynamics in entire its sublimity and beauty.

One should assume that at the basis of electrodynamics it is necessary to place three laws: Coulomb law, the law of the dependence of the electric field of charge on the speed of its motion and the law of the dependence of the electric field of charge on its acceleration. Coulomb law determines the electric field of fixed charge. The presence in the charge of speed leads to increase in its electric field in the direction transverse relative to speed. But the acceleration of charge leads to the induction of additional electric field in the direction longitudinal relative to acceleration. Coulomb law and law of the dependence of the electric field of charge on its acceleration has already been contained in Maxwell's electrodynamics. However, the law of the dependence of the electric field of charge on the speed of its motion, which realizes the concept of scalar- vector potential, is fundamentally new. The association of all these three laws into the united foundation of electrodynamics radically changes its ideology. It makes it possible to examine from the united positions and static interaction of charges, and the laws of power interaction in the case of their mutual motion, and the emission laws and scattering. This approach made it possible to overcome problems 
of electrodynamics described in this monograph the theoretical, to explain SR phase aberration and transverse Doppler effect without the attraction, which could not make within the framework a traditional ideology of electrodynamics.

\section{$\S 19$. Power interaction of the current carrying systems, homopolar induction and the pondermotive forces}

It was already said, that Maxwell equations do not include information about power interaction of the current carrying systems. In the classical electrodynamics for calculating such an interaction it is necessary to calculate magnetic field in the assigned region of space, and then, using a Lorentz force, to find the forces, which act on the moving charges. Obscure a question about that remains with this approach, to what are applied the reacting forces with respect to those forces, which act on the moving charges.

The concept of magnetic field arose to a considerable degree because of the observations of power interaction of the current carrying and magnetized systems. Experience with the iron shavings, which are erected near the magnet poles or around the annular turn with the current into the clear geometric figures, is especially significant. These figures served as occasion for the introduction of this concept as the lines of force of magnetic field. In accordance with third Newton's law with any power interaction there is always a equality of effective forces and opposition, and also always there are those elements of the system, to which these forces are applied. A large drawback in the concept of magnetic field is the fact that it does not give answer to that, counteracting forces are concretely applied to what, since. magnetic field comes out as the independent substance, with which occurs interaction of the moving charges.

Is experimentally known that the forces of interaction in the current carrying systems are applied to those conductors, whose moving charges create magnetic field. However, in the existing concept of power interaction of the current carrying systems, based on the concepts of magnetic field and Lorentz force, the positively harged lattice, which is the frame of conductor and to which are applied the forces, it does not participate in the formation of the forces of interaction. That that the positively charged ions take direct part in the power processes, speaks the fact that in the process of compressing the plasma in transit through its direct current (the so-called pinch effect) it occurs the compression also of ions.

Let us examine this question within the framework of the concept of scalarvector potential. We will consider that the scalar-vector potential of single charge is determined by relationship (16.4), and that the electric fields, created by this potential, act on all surrounding charges, including to the charges positively charged lattices.

Let us examine from these positions power interaction between two parallel conductors (Fig. 14), along which flow the currents. We will consider that $g_{1}^{+}, g_{2}^{+}$ and $g_{1}{ }^{-}, g_{2}{ }^{-}$present the respectively fixed and moving charges, which fall per unit of the length of conductor. 
The charges $g_{1}^{+}, g_{2}{ }^{+}$present the positively charged lattice in the lower and upper conductors. We will also consider that both conductors prior to the start of charges are electrically neutral, i.e., in the conductors there are two systems of the mutually inserted opposite charges with the specific density to $g_{1}{ }^{+}, g_{1}{ }^{-}$and $g_{2}{ }^{+}, g_{2}{ }^{-}$, which electrically neutralize each other.

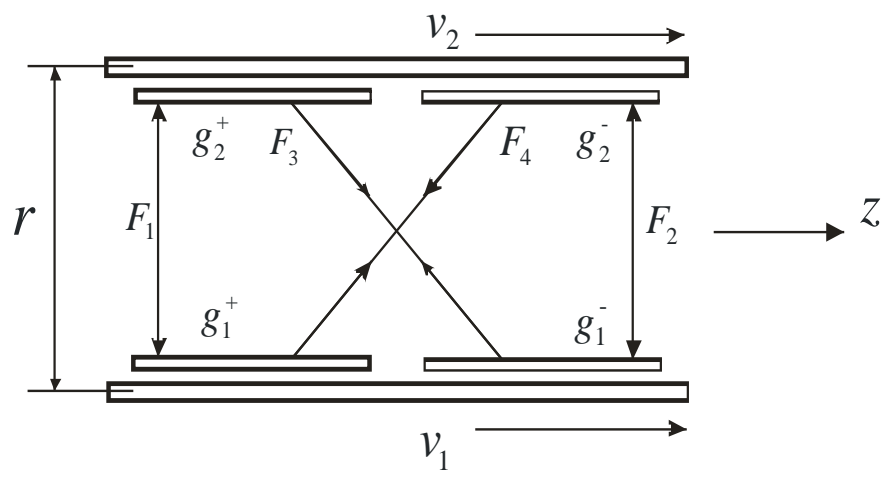

Fig. 14. Schematic of power interaction of the current carrying wires of two-wire circuit taking into account the positively charged lattice

In Fig. 14 these systems for larger convenience in the examination of the forces of interaction are moved apart along the axis $z$. Subsystems with the negative charge (electrons) can move with the speeds of $v_{1}, v_{2}$. The force of interaction between the lower and upper conductors we will search for as the sum of four forces, whose designation is understandable from the figure. The repulsive forces $F_{1}, F_{2}$ we will take with the minus sign, while the attracting force $F_{3}, F_{4}$ we will take with the plus sign.

For the single section of the two-wire circuit of force, acting between the separate subsystems, will be written down

$$
F_{1}=-\frac{g_{1}^{+} g_{2}^{+}}{2 \pi \varepsilon r}, F_{2}=-\frac{g_{1}{ }^{-} g_{2}{ }^{-}}{2 \pi \varepsilon r} \operatorname{ch} \frac{v_{1}-v_{2}}{c}, F_{3}=+\frac{g_{1}^{-} g_{2}{ }^{+}}{2 \pi \varepsilon r} \operatorname{ch} \frac{v_{1}}{c}, F_{4}=+\frac{g_{1}{ }^{+} g_{2}}{2 \pi \varepsilon r} \operatorname{ch} \frac{v_{2}}{c} .
$$

Adding all force components, we will obtain the amount of the composite force, which falls per unit of the length of conductor,

$$
F_{\Sigma}=\frac{g_{1} g_{2}}{2 \pi \varepsilon r}\left(\operatorname{ch} \frac{v_{1}}{c}+\operatorname{ch} \frac{v_{2}}{c}-\operatorname{ch} \frac{v_{1}-v_{2}}{c}-1\right)
$$

In this expression as $g_{1}, g_{2}$ are undertaken the absolute values of charges, and the signs of forces are taken into account in the bracketed expression. For the case $v$ $<<c$, let us take only two first members of expansion in the series $\operatorname{ch} \frac{v}{c}$, i.e., we will consider that $\operatorname{ch} \frac{v}{c} \cong 1+\frac{1}{2} \frac{v^{2}}{c^{2}}$. From relationship (18.2) we obtain

$$
F_{\Sigma 1}=\frac{g_{1} v_{1} g_{2} v_{2}}{2 \pi \varepsilon c^{2} r}=\frac{I_{1} I_{2}}{2 \pi \varepsilon c^{2} r},
$$


where $g_{1}, g_{2}$ are undertaken the absolute values of specific charges, and $v_{1}, v_{2}$ take with its signs.

Since the magnetic field of straight wire, along which flows the current $I$, we determine by the relationship

$$
H=\frac{I}{2 \pi r},
$$

From relationship (18.2) we obtain

$$
F_{\Sigma 1}=\frac{I_{1} I_{2}}{2 \pi \varepsilon c^{2} r}=\frac{H_{1} I_{2}}{\varepsilon c^{2}}=I_{2} \mu H_{1},
$$

where $H_{1}$ - the magnetic field, created by lower conductor in the location of upper conductor.

It is analogous

$$
F_{\Sigma 1}=I_{1} \mu H_{2},
$$

where $\mathrm{H}_{2}$ - the magnetic field, created by upper conductor in the region of the arrangement of lower conductor. These relationships completely coincide with the results, obtained on the basis of the concept of magnetic field.

The relationship (19.3) represents the known rule of power interaction of the current carrying systems, but is obtained it not by the phenomenological way on the basis of the introduction of phenomenological magnetic field, but on the basis of completely intelligible physical procedures, under the assumption that that the scalar potential of charge depends on speed. In the formation of the forces of interaction in this case the lattice takes direct part, which is not in the model of magnetic field. In the model examined are well visible the places of application of force. The obtained relationships coincide with the results, obtained on the basis of the concept of magnetic field and by the axiomatically introduced Lorentz force. In this case is undertaken only first member of expansion in the series $\operatorname{ch} \frac{v}{c}$. For the speeds $v \sim c$ should be taken all terms of expansion. In terms of this the proposed method is differed from the method of calculation of power interactions by the basis of the concept of magnetic field. If we consider this circumstance, then the connection between the forces of interaction and the charge rates proves to be nonlinear. This, in particular it leads to the fact that the law of power interaction of the current carrying systems is asymmetric. With the identical values of currents, but with their different directions, the attracting forces and repulsion become unequal. Repulsive forces prove to be greater than attracting force. This difference is small and is determined by the expression

$$
\Delta F=\frac{v^{2}}{2 c^{2}} \frac{I_{1} I_{2}}{2 \pi \varepsilon c^{2} \varepsilon},
$$

but with the speeds of the charge carriers of close ones to the speed of light it can prove to be completely perceptible.

Let us remove the lattice of upper conductor (Fig. 14), after leaving only free electronic flux. In this case will disappear the forces $F_{1}, F_{3}$, and this will indicate interaction of lower conductor with the flow of the free electrons, which move with 
the speed of $v_{2}$ on the spot of the arrangement of upper conductor. In this case the value of the force of interaction is defined as:

$$
F_{\Sigma}=\frac{g_{1} g_{2}}{2 \pi \varepsilon r}\left(\operatorname{ch} \frac{v_{2}}{c}-\operatorname{ch} \frac{v_{1}-v_{2}}{c}\right)
$$

Lorentz force assumes linear dependence between the force, which acts on the charge, which moves in the magnetic field, and his speed. However, in the obtained relationship the dependence of the amount of force from the speed of electronic flux will be nonlinear. From relationship (19.4) it is not difficult to see that with an increase in $v_{2}$ the deviation from the linear law increases, and in the case, when $v_{2}>v_{1}$, the force of interaction are approached zero. This is very meaningful result. Specifically, this phenomenon observed in their known experiments Thompson and Kauffmann, when they noted that with an increase in the velocity of electron beam it is more badly slanted by magnetic field. They connected the results of their observations with an increase in the mass of electron. As we see reason here another.

Let us note still one interesting result. From relationship (19.3), with an accuracy to quadratic terms, the force of interaction of electronic flux with the rectilinear to determine according to the following dependence:

$$
F_{\Sigma}=\frac{g_{1} g_{2}}{2 \pi \varepsilon r}\left(\frac{v_{1} v_{2}}{c^{2}}-\frac{1}{2} \frac{v_{1}^{2}}{c^{2}}\right)
$$

From expression (19.5) follows that with the unidirectional electron motion in the conductor and in the electronic flux the force of interaction with the fulfillment of conditions $v_{1}=\frac{1}{2} v_{2}$ is absent.

Since the speed of the electronic flux usually much higher than speed of current carriers in the conductor, the second term in the brackets in relationship (19.5) can be disregarded. Then, since

$$
H_{1}=\frac{g_{1} v_{1}}{2 \pi \varepsilon c^{2} r}
$$

we will obtain the magnetic field, created by lower conductor in the place of the motion of electronic flux

$$
F_{\Sigma}=\frac{g_{1} g_{2}}{2 \pi \varepsilon r} \frac{v_{1} v_{2}}{c^{2}}=g_{2} \mu v_{2} H
$$

In this case, the obtained value of force exactly coincides with the value of Lorentz force. Taking into account that

$$
F_{\Sigma}=g_{2} E=g_{2} \mu v_{2} H
$$

it is possible to consider that on the charge, which moves in the magnetic field, acts the electric field $E$, directed normal to the direction of the motion of charge. This result also with an accuracy to of the quadratic terms $\frac{v^{2}}{c^{2}}$ completely coincides with the results of the concept of magnetic field and is determined the Lorentz force, which acts from the side of magnetic field to the flow of the moving electrons. 
As was already said, one of the important contradictions to the concept of magnetic field is the fact that two parallel beams of the like charges, which are moved with the identical speed in one direction, must be attracted. In this model there is no this contradiction already. If we consider that the charge rates in the upper and lower wire will be equal, and lattice is absent, i.e., to leave only electronic fluxes, then will remain only the repulsive force $F_{2}$.

Thus, the moving electronic flux interacts simultaneously both with the moving electrons in the lower wire and with its lattice, and the sum of these forces of interaction it is called Lorentz force. This force acts on the moving electron stream.

Regularly does appear a question, and does create magnetic field most moving electron stream of in the absence compensating charges of lattice or positive ions in the plasma? The diagram examined shows that the effect of power interaction between the current carrying systems requires in the required order of the presence of the positively charged lattice. Therefore most moving electronic flux cannot create that effect, which is created during its motion in the positively charged lattice. At the same time, if we examine two in parallel moving electron streams, then appears the extra force of interaction, which depends on the relative speed of these flows.

Let us demonstrate still one approach to the problem of power interaction of the current carrying systems. The statement of facts of the presence of forces between the current carrying systems indicates that there is some field of the scalar potential, whose gradient ensures the force indicated. But that this for the field? Relationship (19.3) gives only the value of force, but he does not speak about that, the gradient of what scalar potential ensures these forces. We will support with constants the currents $I_{1}, I_{2}$, and let us begin to draw together or to move away conductors. The work, which in this case will be spent, and is that potential, whose gradient gives force. After integrating relationship (18.3) on $r$, we obtain the value of the energy:

$$
W=\frac{I_{1} I_{2} \ln r}{2 \pi \varepsilon c^{2}} .
$$

This energy, depending on that to move away conductors from each other, or to draw together, can be positive or negative. When conductors move away, then energy is positive, and this means that, supporting current in the conductors with constant, generator returns energy. This phenomenon is the basis the work of all electric motors. If conductors converge, then work accomplish external forces, on the source, which supports in them the constancy of currents. This phenomenon is the basis the work of the mechanical generators of emf.

Relationship for the energy can be rewritten and thus:

$$
W=\frac{I_{1} I_{2} \ln r}{2 \pi \varepsilon c^{2}}=I_{2} A_{z 1}=I_{1} A_{z 2},
$$

where

$$
A_{z 1}=\frac{I_{1} \ln r}{2 \pi \varepsilon c^{2}}
$$


is $z$ - component of vector potential, created by lower conductor in the location of upper conductor, and

$$
A_{z 2}=\frac{I_{2} \ln r}{2 \pi \varepsilon c^{2}}
$$

is $z$-component of vector potential, created by upper conductor in the location of lower conductor.

The approach examined demonstrates that large role, which the vector potential in questions of power interaction of the current carrying systems and conversion of electrical energy into the mechanical plays. This approach also clearly indicates that the Lorentz force is a consequence of interaction of the current carrying systems with the field of the vector potential, created by other current carrying systems. Important circumstance is the fact that the formation of vector potential is obliged to the dependence of scalar potential on the speed. This is clear from a physical point of view. The moving charges, in connection with the presence of the dependence of their scalar potential on the speed, create the scalar field, whose gradient gives force. But the creation of any force field requires expenditures of energy. These expenditures accomplishes generator, creating currents in the conductors. In this case in the surrounding space is created the special field, which interacts with other moving charges according to the special vector rules, with which only scalar product of the charge rate and vector potential gives the potential, whose gradient gives the force, which acts on the moving charge. This is a Lorentz force.

In spite of simplicity and the obviousness of this approach, this simple mechanism up to now was not finally realized. For this reason the Lorentz force, until now, was introduced in the classical electrodynamics by axiomatic way.

Let us examine the still one interesting consequence, which escapes from the given examination. If we as the planes of long line use an superconductor, then the magnetic field on its surface, equal to specific current, can be determined from the relationship:

where $\lambda=\sqrt{\frac{m}{n e^{2} \mu}}$ - depth of penetration of magnetic field into the superconductor.

If we substitute the value of depth of penetration into relationship (19.6), then we will obtain the unexpected result:

$$
H=v \sqrt{\frac{n m}{\mu}} .
$$

Occurs that the magnetic field strength completely does not depend on the magnitude of the charge of current carriers, but it depends on their mass. Thus, the density energy of magnetic fields

$$
W_{H}=\frac{1}{2} \mu H^{2}=\frac{n m v^{2}}{2}
$$


is equal to density of the kinetic energy of charges. But the magnetic field, connected with the motion of current carriers in the surface layer of superconductor, exists not only on its surface, also, in the skin-layer. Volume, occupied by magnetic fields, incommensurably larger than the volume of this layer. If we designate the length of the line, depicted in Fig. 2 as $l$, then the volume of skin-layer in the superconductive planes of line will compose $2 l b \lambda$. Density energy of magnetic fields on in this volume we determine from the relationship:

$$
W_{H, \lambda}=n m v^{2} l b \lambda,
$$

however, density energy of magnetic fields on, accumulated between the planes of line, it will comprise:

$$
W_{H, a}=\frac{n m v^{2} l b a}{2}=\frac{1}{2} l b a \mu_{0} H .
$$

If one considers that the depth of penetration of magnetic field in the superconductors composes several hundred angstroms, then with the macroscopic dimensions of line it is possible to consider that the total energy of magnetic fields on in it they determine by relationship (19.8). Therefore, the formation of magnetic fields on $H$ between the planes of line, which appear in connection with the motion of charges in the skin-layer, it requires the same expenditures of energy, as if entire volume of line was filled with the particles, which move with the speed of $v$, whose density and mass compose respectively $n$ and $m$.

Is obvious that the effective mass of electron in comparison with the mass of free electron grows in this case into $\frac{a}{2 \lambda}$ of times. This is the consequence of the fact that the mechanical electron motion leads not only to the accumulation of their kinetic energy in the skin-layer, but in the line also occurs accumulation and potential energies, whose gradient gives the force, which acts on the conducting planes of line. Thus, becomes clear nature of such parameters as inductance and the effective mass of electron, which in this case depend, in essence, not from the mass of free electrons, but from the configuration of conductors, on which the electrons move.

The homopolar induction was discovered still by Faraday almost 200 years ago, but in the classical electrodynamics of final answer to that as and why work some constructions of unipolar generators, there is no up to now. Is separately incomprehensible the case, when there is a revolving magnetized conducting cylinder, during motion of which between the fixed contacts, connected to its axis and generatrix, appears emf. Is still more incomprehensible the case, when together with the cylindrical magnet revolves the conducting disk, which does not have galvanic contact with the magnet, but fixed contacts are connected to the axis of disk and its generatrix. In some sources it is indicated that the answer can be obtained within the framework SR, but there are no concrete references, as precisely SR explain the cases indicated. It will be further shown that the concrete answers to all these questions can be obtained within the framework the concept of the dependence of the scalar potential of charge on its relative speed.

Let us examine the case, when there is a single long conductor, along which flows the current. We will as before consider that in the conductor is a system of 
the mutually inserted charges of the positive lattice $g^{+}$and free electrons $g^{-}$, which in the absence current neutralize each other (Fig.15).

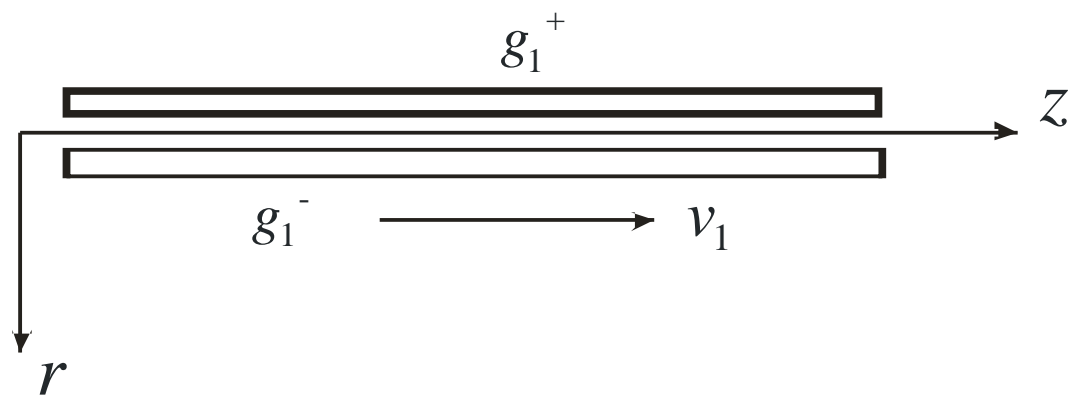

Fig. 15. Section is the conductor, along which flows the current

The electric field, created by rigid lattice depending on the distance $r$ from the center of the conductor that is located along the axis $z$ it takes the form

$$
E^{+}=\frac{g^{+}}{2 \pi \varepsilon r}
$$

We will consider that the direction of the vector of electric field coincides with the direction $r$. If electronic flux moves with the speed $v_{1}$, then the electric field of this flow is determined by the equality

$$
E^{-}=-\frac{g^{-}}{2 \pi \varepsilon r} \operatorname{ch} \frac{v_{1}}{c} \cong-\frac{g^{-}}{2 \pi \varepsilon r}\left(1+\frac{1}{2} \frac{v_{1}^{2}}{c^{2}}\right)
$$

Adding (19.9), (19.10), we obtain:

$$
E^{-}=-\frac{g^{-} v_{1}^{2}}{4 \pi \varepsilon c^{2} r}
$$

This means that around the conductor with the current is an electric field, which corresponds to the negative charge of conductor. However, this field has insignificant value, since in the real conductors $v \leq c$. This field can be discovered only with the current densities, which can be achieved in the superconductors, which is experimentally confirmed in works.

Let us examine the case, when very section of the conductor, on which with the speed $v_{1}$ flow the electrons, moves in the opposite direction with speed $v$ (Fig. 16). In this case relationships (19.9) and (19.10) will take the form

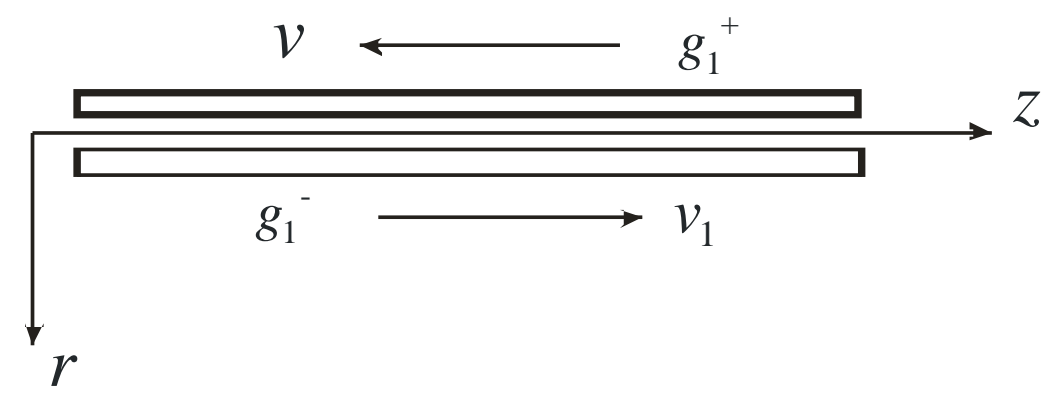

Fig. 16. Moving conductor with the current 


$$
\begin{gathered}
E^{+}=\frac{g^{+}}{2 \pi \varepsilon r}\left(1+\frac{1}{2} \frac{v^{2}}{c^{2}}\right) \\
E^{-}=-\frac{g^{-}}{2 \pi \varepsilon r}\left(1+\frac{1}{2} \frac{\left(v_{1}-v\right)^{2}}{c^{2}}\right)
\end{gathered}
$$

Adding (18.20), (18.21), we obtain

$$
E^{+}=\frac{g}{2 \pi \varepsilon r}\left(\frac{v_{1} v}{c^{2}}-\frac{1}{2} \frac{v_{1}^{2}}{c^{2}}\right) .
$$

In this relationship as the specific charge is undertaken its absolute value. since the speed of the mechanical motion of conductor is considerably more than the drift velocity of electrons, the second term in the brackets can be disregarded. In this case from (19.13) we obtain

$$
E^{+}=\frac{g v_{1} v}{2 \pi \varepsilon c^{2} r} .
$$

The obtained result means that around the moving conductor, along which flows the current, with respect to the fixed observer is formed the electric field, determined by relationship (19.14), which is equivalent to appearance on this conductor of the specific positive charge of the equal

$$
g^{+}=\frac{g v_{1} v}{c^{2}} .
$$

If we conductor roll up into the ring and to revolve it then so that the linear speed of its parts would be equal $v$, then around this ring will appear the electric field, which corresponds to the presence on the ring of the specific charge indicated. But this means that the revolving turn, which is the revolving magnet, acquires specific electric charge on wire itself, of which it consists. During the motion of linear conductor with the current the electric field will be observed with respect to the fixed observer, but if observer will move together with the conductor, then such fields will be absent.

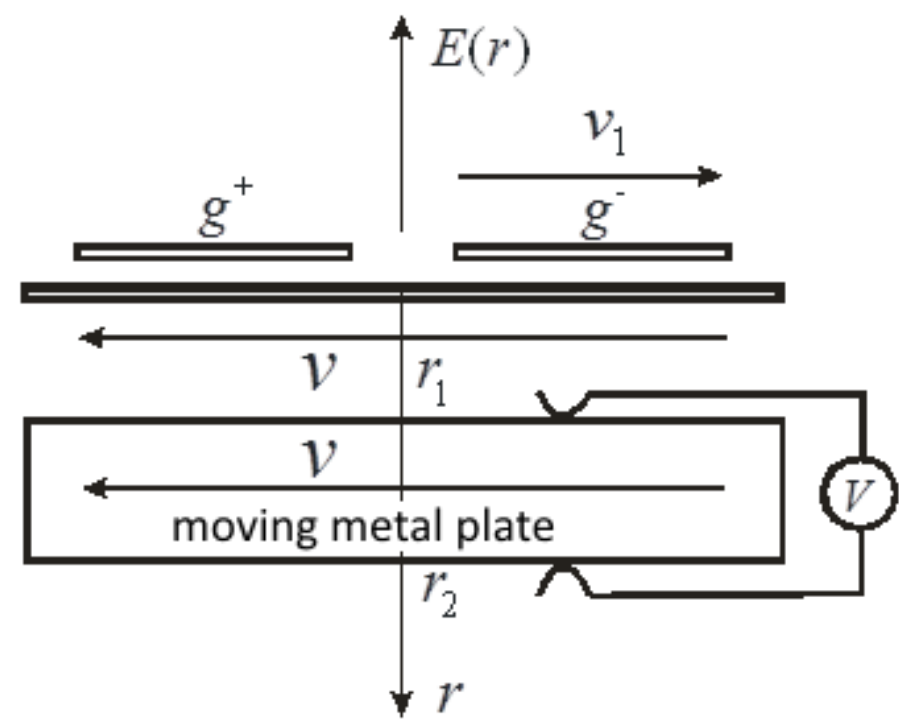

Fig. 17. Diagram of formation emf homopolar induction. 
As is obtained the homopolar induction, with which on the fixed contacts a potential difference is obtained, it is easy to understand from Fig. 17.

We will consider that $r_{1}, r_{2}$ of the coordinate of the points of contact of the tangency of the contacts, which slide along the edges of the metallic plate, which moves with the same speed as the conductor, along which flows the current. Contacts are connected to the voltmeter, which is also fixed. Then, it is possible to calculate a potential difference between these contacts, after integrating relationship (19.14):

$$
U=\frac{g v_{1} v}{2 \pi \varepsilon c^{2}} \int_{r_{1}}^{r_{2}} \frac{d r}{r}=\frac{g v_{1} v}{2 \pi \varepsilon c^{2}} \ln \frac{r_{2}}{r_{1}} .
$$

But in order to the load, in this case to the voltmeter, to apply this potential difference, it is necessary sliding contacts to lock by the cross connection, on which there is no potential difference indicated. But since metallic plate moves together with the conductor, a potential difference is absent on it. It serves as that cross connection, which gives the possibility to convert this composite outline into the source emf. with respect to the voltmeter.

Now it is possible wire to roll up into the ring (Fig. 18) of one or several turns, and to feed it from the current source. Moreover contacts 1 should be derived on the collector rings, which are located on the rotational axis and to them joined the friction fixed brushes. Thus, it is possible to obtain the revolving magnet. In this magnet should be placed the conducting disk with the opening, which revolves together with the turns of the wire, which serves as magnet, and with the aid of the fixed contacts, that slide on the generatrix of disk, tax voltage on the voltmeter. As the limiting case it is possible to take continuous metallic disk and to connect sliding contacts to the generatrix of disk and its axis. Instead of the revolving turn with the current it is possible to take the disk, magnetized in the axial direction, which is equivalent to turn with the current, in this case the same effect will be obtained.

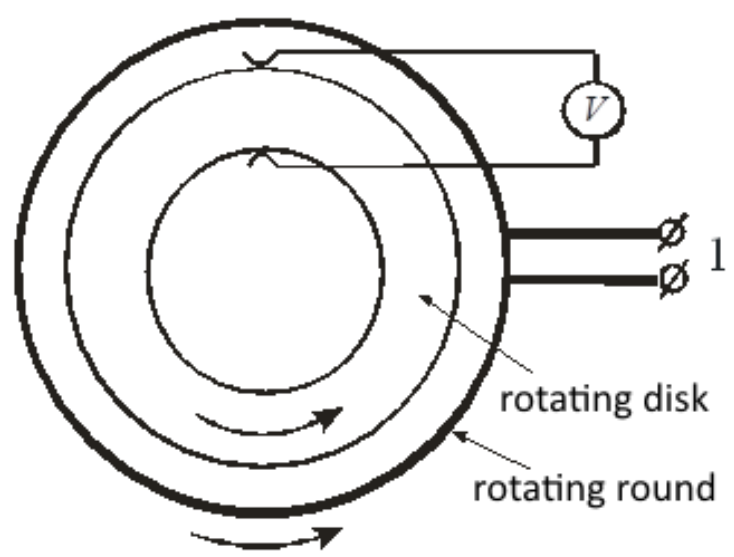

Fig. 18. Schematic of unipolar generator with the revolving turn with the current and the revolving conducting ring. 
The case with the fixed magnet and the revolving conducting disk is characterized by the diagram, depicted in Fig. 19, if the conducting plate was rolled up into the ring.

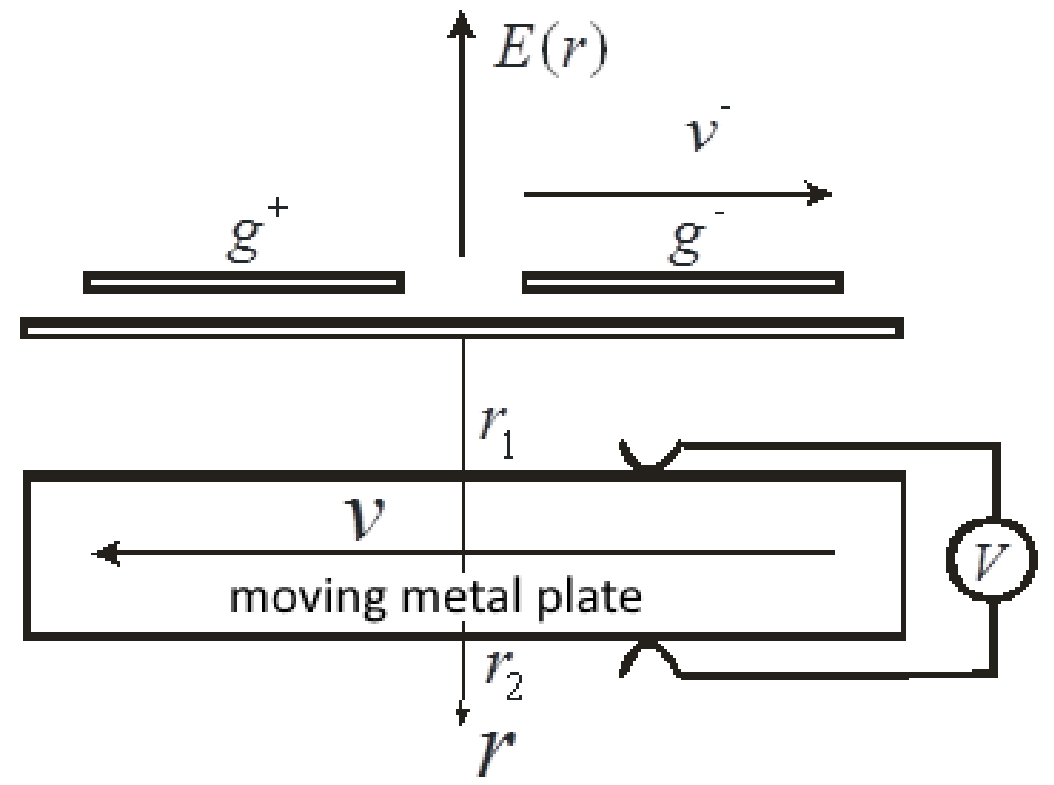

Fig. 19. The case of fixed magnet and revolving disk.

Different combinations of the revolving and fixed magnets and disks are possible. In this case the following relationships are fulfilled:

The electric field, generated in the revolving disk by the electrons, which move along the conductor, is determined by the relationship

$$
E^{-}=-\frac{g^{-}}{2 \pi \varepsilon r} \operatorname{ch} \frac{v_{1}-v}{c}=-\frac{g^{-}}{2 \pi \varepsilon r}\left(1+\frac{1}{2} \frac{\left(v_{1}-v\right)^{2}}{c^{2}}\right),
$$

and by the fixed ions

$$
E^{+}=\frac{g^{+}}{2 \pi \varepsilon r} \operatorname{ch} \frac{v}{c}=\frac{g^{-}}{2 \pi \varepsilon r}\left(1+\frac{1}{2} \frac{v^{2}}{c^{2}}\right) .
$$

The summary tension of electric field in this case will comprise

$$
E_{\Sigma}=\frac{g}{2 \pi \varepsilon r}\left(\frac{v v_{1}}{c^{2}}\right),
$$

and a potential difference between the points $r_{1}$ and $r_{2}$ in the coordinate system, which moves together with the plate, will be equal

$$
U=\frac{g\left(r_{2}-r_{1}\right)}{2 \pi \varepsilon r}\left(\frac{v v_{1}}{c^{2}}\right) .
$$

Since in the fixed with respect to the magnet of the circuit of voltmeter the induced potential difference is absent, the potential difference indicated will be equal by the electromotive force of the generator examined. As earlier moving conducting plate can be rolled up into the disk with the opening, and the wire, along which flows the current into the ring with the current, which is the equivalent of the magnet, magnetized in the end direction. 
Thus, the concept of the dependence of the scalar potential of charge on the relative speed gives answers to all presented questions and SR here it is not necessary.

From these positions it is possible to examine the ponderomotive action of electrical and magnetic fields on to any interface. Current in the region of boundary must be scalar multiplied by the vector potential. The gradient of this work will give the forces, which act on the surface. With this approach calculation of the dependence of the potential gradient energy on the coordinate gives information about the internal stresses, which act in the region of boundary.

Is most easy this it is possible to understand based on the example of superconductors, EM of the waves or presence on their surface of constant magnetic or electrical fields on with the drop on them. In the superconductors the current density is unambiguously connected with the vector potential, and the work of current to the vector potential is potential energy. But since currents in the superconductor diminish exponentially, potential energy of these currents diminishes thus, and the potential gradient energy in the surface layer and On the Border superconductor is the reason for the appearance of ponderomotive forces. By here what defined by example means magnetic field and the incident electromagnetic wave exerts pressure on the surface of superconductor.

Potential electric current energy, which flow in the superconductor is determined by the relationship

$$
W=\frac{1}{2} \mu \int \mathbf{j} \mathbf{A} d V .
$$

Current density in the superconductor changes according to the law

$$
\mathbf{j}(z)=\mathbf{j}(0) e^{-\frac{z}{\lambda}} .
$$

According to the same law changes the vector potential $\mathbf{A}$. Thus

$$
W=\frac{1}{2} \mu \int \mathbf{j} \mathbf{A} d V=\frac{1}{2} \mu \int \mathbf{j}(0) \mathbf{A}(0) e^{-\frac{2 z}{\lambda}} d z
$$

Consequently, ponderomotive force will have a value

$$
\mathbf{F}=-\operatorname{grad} W=\frac{1}{2} \mu \mathbf{j}(0) \mathbf{A}(0) e^{-\frac{2 z}{\lambda}} .
$$

In the superconductor the current density is determined by the relationship

$$
\mathbf{j}=-\frac{\mu}{L_{k}} \mathbf{A},
$$

where $L_{k}=\frac{m}{n e^{2}}$ - the kinetic inductance of charges, $\lambda=\sqrt{\frac{L_{k}}{\mu}}$ - London depth of penetration.

Therefore

$$
\mathrm{F}=\frac{1}{2} \mathrm{j}^{2}(0) L_{k} e^{-\frac{2 z}{\lambda}}
$$

This force is equal on the surface

$$
F=\frac{1}{2} j^{2}(0) L_{k} .
$$


The magnetic field on its surface of superconductor, equal to specific current, can be determined from the relationship by the current, which flows under the surface

$$
H=I=\int j(0) e^{-\frac{z}{\lambda}} d z=\lambda j(0) .
$$

Is consequently the force applied to the surface of superconductor

$$
\mathrm{F}=\frac{1}{2} \mathrm{j}^{2}(0) L_{k}=\frac{1}{2} H^{2} \frac{L_{k}}{\lambda^{2}}=\frac{1}{2} \mu H^{2} .
$$

If electromagnetic wave is incident on the surface of superconductor, $H=H_{0} \sin \omega t$, then it generates on its surface the specific current

$$
I=H=H_{0} \sin \omega t .
$$

Ponderomotive force in this case will be equal

$$
\mathrm{F}=\frac{1}{2} \mu H_{0}^{2} \sin ^{2} \omega t
$$

The constant component of this force to be determined by the relationship

$$
\mathrm{F}=\frac{1}{4} \mu H_{0}^{2}
$$

Since the superconductor does not absorb energy of electromagnetic wave, it will be completely reflected. This is equivalent to the elastic reflection of material object from the body surface, whose mass is considerably greater than the mass of the falling body.

\section{§ 20. Experimental confirmation of the dependence of the scalar potential of charge on its relative speed}

If we in relationship (19.1) place $g_{2}^{+}=0$ and $v_{2}=0$,, i.e., to examine the case of interaction of the lower conductor, along which flows the current, with the fixed charge of the upper conductor $g_{2}^{-}$in the absence of lattice, then for the force of in teraction we will obtain: $F_{\Sigma 2}=-\frac{1}{2} \frac{g_{1} g_{2} v_{1}^{2}}{2 \pi \varepsilon c^{2} r}$.

This means that the current when flows along the conductor, it ceases to be electrically neutral, and around it must be formed the radial static electric field

$$
E_{\perp}=-\frac{g_{1} v_{1}^{2}}{4 \pi \varepsilon c^{2} r}
$$

which is equivalent to appearance on the lower conductor of additional negative potential, which is, in turn, equivalent to appearance on this conductor of the additional specific static charge

$$
g=-2 g_{1} \frac{v_{1}^{2}}{c^{2}} .
$$

This fact attests to the fact that the adoption of the concept of scalar- vector potential indicates the acknowledgement of the dependence of charge on the speed. 
However, up to now no one obtained experimental confirmation the validity of relationships (20.1) and (20.2).

When by Faraday and by Maxwell were formulated the fundamental laws of electrodynamics, to experimentally confirm relationship (20.1) it was impossible, since. the current densities, accessible in the usual conductors, are too small for the experimental detection of the effect in question. Thus, position about the independence of scalar potential and charge from the speed and the subsequent introduction of magnetic field they were made volitional way on the phenomenological basis.

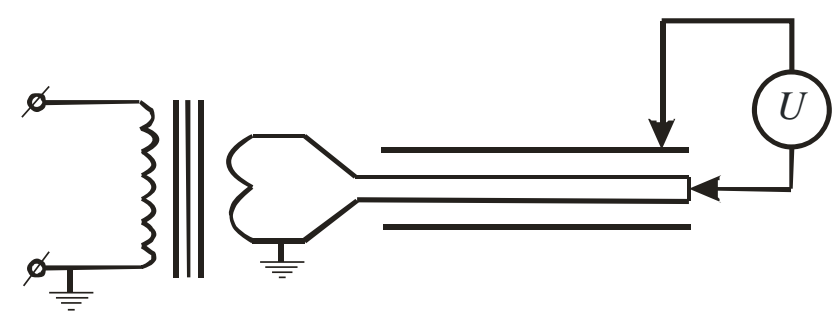

Fig. 20. Experimental confirmation of the dependence of the scalar potential of charge on its relative speed

The current density, which can be achieved in the superconductors, make it possible to experimentally detect the electric fields, determined by relationship (20.1) [32]. If such fields will be discovered, then this means that the scalar potential of charge depends on its relative speed.

Let us examine setting the experiment, which must give answer to the presented questions. The diagram of experiment is depicted in Fig. 20. If the folded in half superconductive wire (we will call its bifilar) to surround by the conducting cylinder and to introduce into it current in an induction manner, then in the case the dependence of charge on the speed the electrometer with the high internal resistance, connected between the cylinder and the wire, must show the presence of a potential difference. The noncontact induction introduction of current adapts with that purpose in order to exclude the presence of contact potential differences with the contact introduction of current. The difficulty of conducting this experiment consists in the fact that the input capacitance of the electrometer (usually several ten Pico farads) it will be considerably more than the capacity between the bifilar loop and the cylinder. Since we measure not emf, but a potential difference, with the connection to this device of the input capacitance of electrometer the charge, induced on the cylinder to redistribute between both capacities. If we consider that an initial potential difference between the loop and the cylinder was $U_{1}$, and the capacity between them composed $C_{1}$, then with the connection between loop and cylinder of the additional tank of the electrometer $C_{2}$ a potential difference $U_{2}$ to be determined by the relationship:

$$
U_{2}=\frac{C_{1} U_{1}}{C_{1}+C_{2}}=k_{1} U_{1} .
$$

Is obvious that if $C_{1} \ll<C_{2}$, then $U_{2}<<U_{1}$. In the final analysis it turns out that in order to obtain a maximum voltage drop across electrometer itself should be in- 
creased the capacity between the loop and the cylinder, increasing the length of entire construction.

Let us begin from the determination of the expected effect the calculation of the parameters of the measuring system, intended for detecting the expected effect.

If is located the plane layer of charges with the density $n$ and the thickness $\lambda$, the like both sides from this layer it is created the electric field:

$$
E_{\perp}=\frac{1}{2} \frac{n e \lambda}{\varepsilon_{0}} .
$$

Thus far this layer of charges does not move its electric field is completely compensated by the positive charges of lattice. But, when layer begins to move, is created additional electric field equal

$$
\Delta E \cong \frac{1}{2} E_{\perp} \frac{v^{2}}{c^{2}} .
$$

The speed of the motion of charges is connected with the magnetic field with the relationship:

$$
H=n e v \lambda .
$$

If, speed $v$, obtained from this relationship was substituted in (20.4), then we will obtain

$$
\Delta E_{\perp}=\frac{1}{2} \frac{H^{2}}{\varepsilon_{0} n e \lambda c^{2}}=\frac{1}{2} \frac{\mu_{0} H^{2}}{n e \lambda} .
$$

For enumerating a maximally expected magnitude of effect as $H$ should be taken the value of critical field for this type of superconductor.

Let us calculate the maximum magnitude of this effect for the case of superconductive niobium, after assuming: $H_{c}=1,5 \cdot 10^{5} \frac{A}{M}, \lambda \cong 10^{-7} \mathrm{M}, n \cong 3 \cdot 10^{28} \frac{1}{\mathrm{M}^{3}}$. With such values of the parameters indicated we obtain $\Delta E_{\perp} \cong 3 \frac{B}{M}$. We will consider that the diameter $d$ bifilar loop composes the doubled value of the diameter of the utilized superconductive wire with a diameter $0,25 \mathrm{~mm}$. If we take the diameter $D$ the cylinder of equal $10 \mathrm{~mm}$, then a potential difference between the loop and the cylinder will comprise: $U=\Delta E_{\perp} \frac{d}{2} \ln \frac{D}{d} \cong 3 m B$.The linear capacity of coaxial there will be $C_{0} \cong 15 \frac{n \Phi}{M}$.

In conducting the experiments at our disposal was located vibrating reed electrometer with a input capacitance $\sim 60 \mathrm{pF}$ and the sensitivity $\sim 1 \mathrm{mV}$. In order to ensure at least the same capacity of the coaxial (in this case a voltage drop across the capacity of electrometer after its connection to the coaxial it will be $1.5 \mathrm{mV}$ ) it is necessary to take the length of the coaxial of 4 meters. Certainly, for the technical reasons it is difficult to cool this coaxial to helium temperatures and furthermore and effect itself proves to be insufficient for its reliable measurement. Therefore the magnitude of effect must be increased at least 100 times. This can be carried out, after increasing a quantity of central cores of coaxial, after bringing it to 
two hundred, for which to be required of 400 meters of wire. Certainly, in this case it is necessary to increase the diameter of its cylindrical part. It is possible to again produce calculation, but use of an experimental model with the coaxial of this size nevertheless unacceptably in view of its unwieldiness, although the possibility of the precise calculation of the expected effect is the great advantage of this solution. In this case us even does not so much interest the precise agreement of calculated and experimental data, as reliable detection of effect itself. Therefore experimental model was created according to another diagram. For purposes the introduction of current into the superconductive winding with the small inductance was used the cooled to helium temperatures transformer with the iron core. Using as the secondary winding of transformer the superconductive winding, connected with the solenoid, it is possible without the presence of galvanic contacts to introduce current into it. In the transformer was used ring-shaped core made of transformer steel with a cross section $9 \mathrm{~cm}^{2}$. The primary and secondary windings of transformer were wound by niobium-titanium wire with the copper coating and contained 150 and 10 turns respectively. Thus, transformer has a transformation ratio 15 . The wire diameter composed $0.25 \mathrm{~mm}$. The secondary winding of transformer is connected in series with the solenoid with the small inductance, which is wound bifilar and contains 2448 turns of the same wire. The overall length of coil composes 910 m.

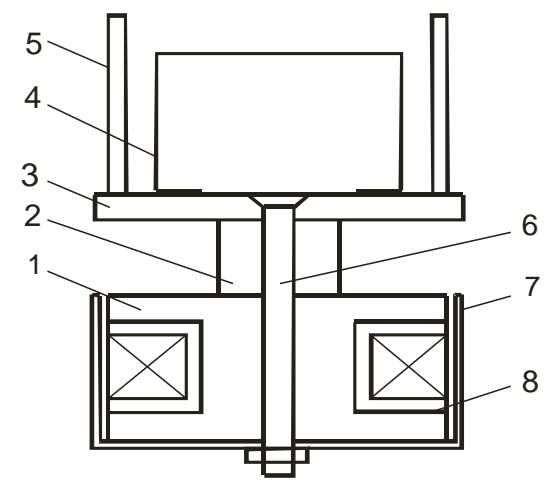

Fig. 21 Construction of the low-inductive superconductive solenoid

The ends of solenoid and secondary winding of transformer are welded with the aid of the laser welding. Solenoid is wound on the body from Teflon resin. Inside and outside diameter of the winding of solenoid 35 and $90 \mathrm{~mm}$ of respectively, the width of the coil $30 \mathrm{~mm}$. To the midpoint of solenoid is connected internal wiring of the coaxial, which emerges outside cryostat, the same coaxial is connected also to the screen of solenoid. The construction of low-inductive solenoid is shown in Fig. 21

By numbers in the figure are designated the following elements: 1 - aluminum body, 2 - Teflon bushing, 3 - Teflon disk, 4- clamp, 5 - counter, 6 - bolt, 7 - copper screen, 8 -Teflon body is eighth. Solenoid is wound on Teflon body 8 , which is concluded in aluminum body 1 . Outside solenoid is surrounded by copper screen 7 , which together with body 1 is the screen of solenoid. To body 1 by means of bolt 6 and Teflon bushing 2 is fastened Teflon disk 3, on which is installed clamp 4 . The turns of the secondary winding of transformer cover clamp 4, through which, with- 
out concerning it, is passed the magnetic circuit of transformer. Entire construction is attached to the transformer by means of counters 5. Transformer together with the solenoid is placed in the tank of helium cryostat. The diagram of the connection of coaxial to the solenoid is shown in Fig. 22.

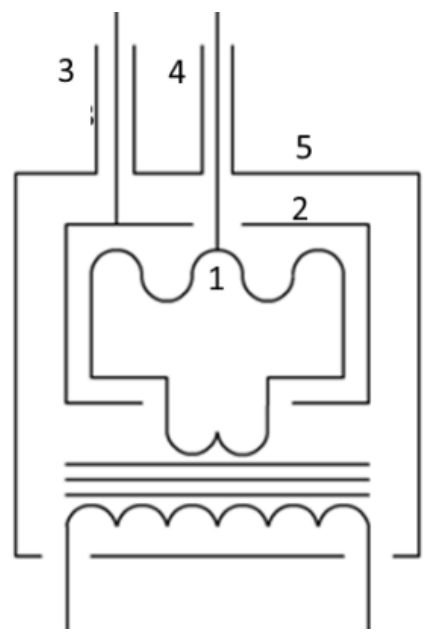

Fig. 22. Diagram of connection of low-inductive solenoid

By the figure are accepted the following designations: 1 - solenoid, 2 - the screen of solenoid, 3,4 - coaxial, 5 - the common screen, which the helium tank is. Resistance between the grounded elements, the screen of solenoid and solenoid itself composes not less than $10^{14} \mathrm{Ohm}$. The elements, utilized in the construction, had the following capacities relative to the earth: the coaxial $3-44 \mathrm{pF}$, coaxial $4-27$ $\mathrm{pF}$, capacity screen - the earth it comprises - $34 \mathrm{pF}$, capacity screen-solenoid compose $-45 \mathrm{pF}$, as the electrometer was used by capacitive vibrating reed electrometer with a input capacitance $60 \mathrm{pF}$ and a input resistance $10{ }^{14} \mathrm{Ohm}$.

During this construction of the superconductive solenoid and its surrounding screen it cannot be produced the precise calculation of electrostatic fields on, that appear around the solenoid to, however, establish the presence of effect itself, this construction allows.

With the measurements electrometer was connected directly to the screen by means of coaxial 4 , and the midpoint of the superconductive solenoid by means of coaxial 3 was grounded. Current into the primary winding of transformer was introduced from the source of direct current, indication of electrometer in this case they did not depend on direction of flow. With the strengths of introduced current $\sim 9$ A occurred the spontaneous discharge of the indications of electrometer. This means that the current in the winding of solenoid reached its critical value, and winding converted to normal state. Iron core in this case seized magnetic flux, also, with the decrease of the current introduced into the solenoid, the curve of the dependence of the measured potential on the current was repeated, and potential reached its maximum value with current zero.

The obtained experimental dependence of the measured potential difference is given in Fig. 23. 
Thus, experimental results indicate that the value of scalar potential, and, therefore, also charge depends on speed.

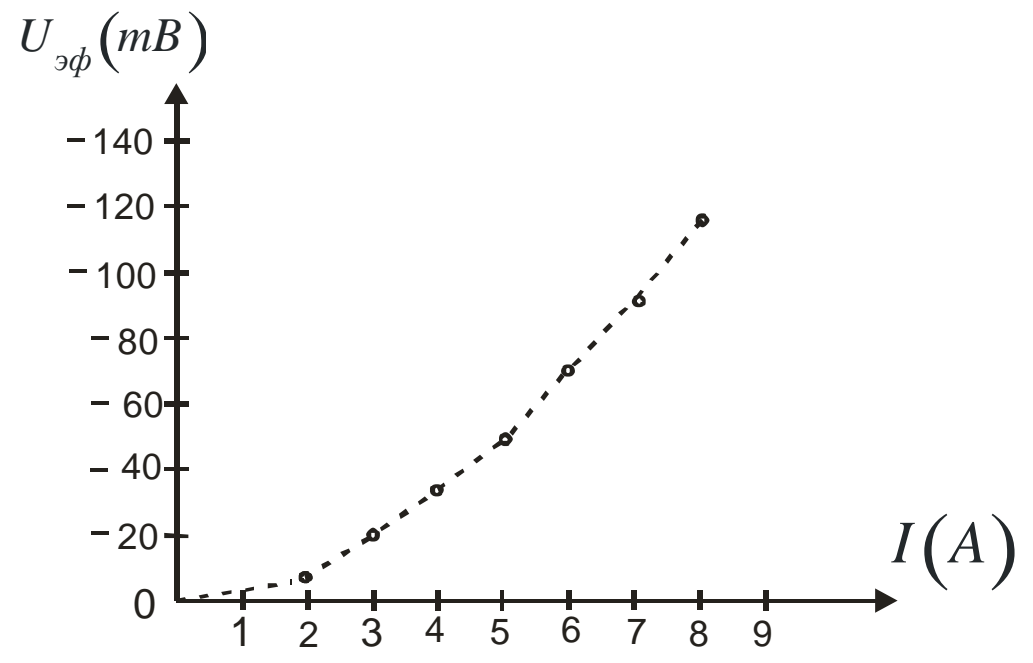

Fig. 23. Dependence of the given potential difference between the screen and the low-inductive solenoid on the current in its winding

However in this diagram of experiment occurs the direct galvanic connection of electrometer to the superconductive solenoid. This can cause questions, but are not the reason for the appearance of a potential difference between the solenoid and the screen some contact phenomena in the place of the contact of wire, which connects electrometer with the solenoid? The experiments with the superconductive niobium torus were carried out for the answer to this question. The diagram of experiment is shown in Fig. 24.

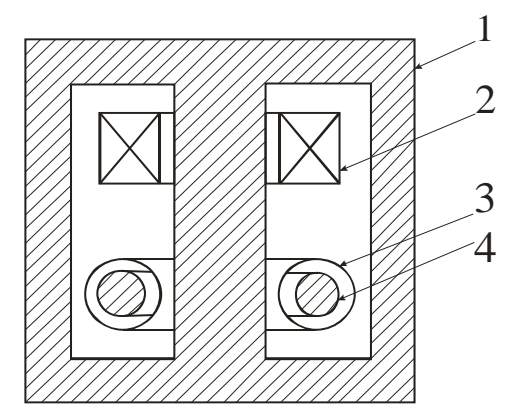

Fig. 24. Diagram of experiment with the superconductive torus

If we inside the conducting screen arrange the second conducting screen, and between them let us connect electrometer, then charge when will appear inside the internal screen, a potential difference will appear between the internal and external screen. In the experiment, as external screen 1, the yoke of transformer, made from transformer steel, was used. On the central rod of this yoke was located primary winding with 2, wound by niobium-titanium wire, which contains 1860 turns. Torus-shaped metal screen 3 , made from copper, was located on the same rod. Torus 4 , made from niobium, was located inside this screen. The outer diameter of niobium torus was $76 \mathrm{~mm}$, and internal $49 \mathrm{~mm}$. Transformer was placed in the tank of helium cryostat and was cooled to the helium temperature, in this case the yoke of 
transformer and helium tank were grounded. The current was induced during the introduction of direct current into the primary winding of transformer in the superconductive torus, and electrometer fixed the appearance between screen 3 and yoke of transformer a potential difference $U$. This means that the niobium torus, located inside screen 3 during the introduction into it of direct current ceases to be electrically neutral. The constant value current in the superconductive torus 1860 times exceeded the current, introduced into the primary winding of transformer.

The dependence of a potential difference $U$ on the current $I$, introduced into the primary winding of transformer, it is shown in Fig. 25.

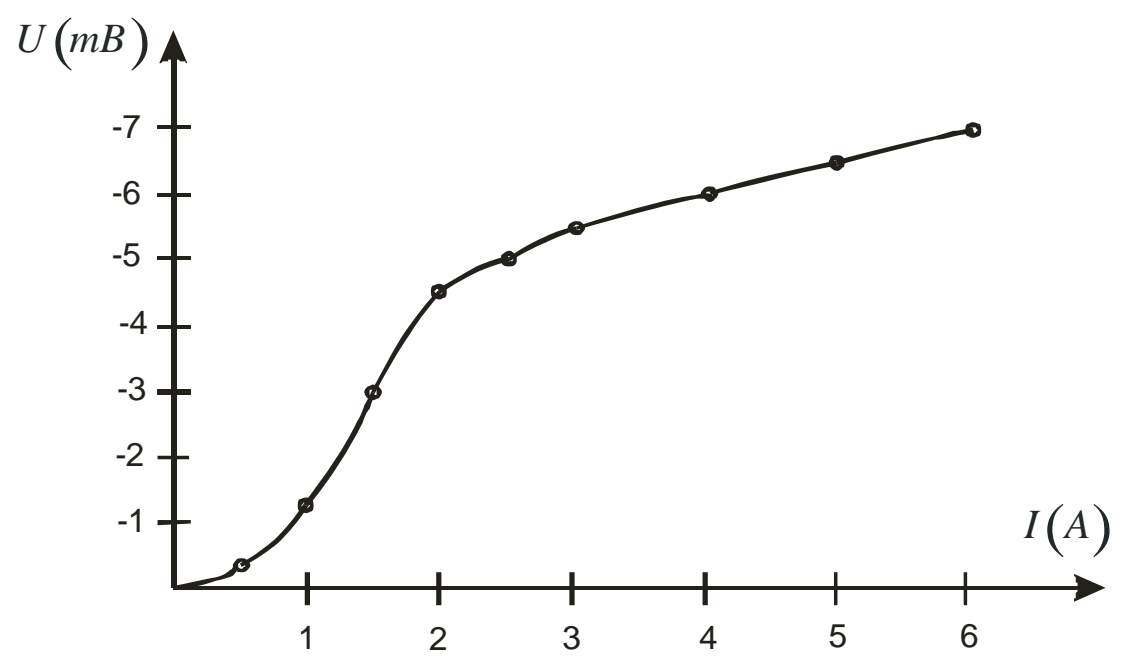

Fig. 25. Dependence of a potential difference boundary by screen 3 by the yoke of transformer 1 on the current, introduced into the primary winding of transformer

The obtained values of a potential difference, in comparison with the case of the superconductive wire winding, proved to be considerably smaller, this is connected with the considerably smaller surface of torus, in comparison with the surface of wire winding. The form of the dependence of a potential difference on the introduced current also strongly differs. Quadratic section is observed only in the very small initial section up to the values of currents into $2 \mathrm{~A}$, introduced into the primary winding. Further this dependence becomes rectilinear with small angle of inclination with respect to the $\mathrm{x}$-axis. It was not observed moreover of stalling the indications of electrometer in this case.

With which are connected such differences in the behavior of a potential difference in comparison with the wire version? In the case of wire solenoid the superconductive current is evenly distributed over the surface of wire and reaches its critical value in all its sections of surface simultaneously, with which and is connected the simultaneous passage of the entire winding of solenoid into the normal state, with the reaching in the wire of the critical value of current.

In the case of torus the process of establishing the superconductive current on its surface occurs differently. That introduced into the direct current superconducting torus is very unevenly distributed over its surface. Maximum current densities occur on the internal surface of torus, and they are considerably less on the periphery. With this is connected the fact that the internal surfaces of torus begin to convert to 
normal state earlier than external. The process of passing the torus into the normal state occurs in such a way that with an increase of the current in the torus into the normal state pass the first interior and normal phase begins to be moved from the interior to the external. Process lasts until entire torus passes into the normal state. But why in this case up to the moment of passing the torus into the normal state does not occur the discharge of current, as it takes place in the case of wire solenoid? This niobium is connected with the fact that the superconductor of the second kind, and it immediately abruptly does not convert to normal state, but he has the sufficiently significant region of current densities, with which it is in the mixed state, when Abrikosov vortices penetrate inside the massive conductor. The circumstance that the indications of electrometer do not have a discharge of indications, he indicates that the superconductive torus is in the mixed state, but the presence of the vortex of the structures in it, which also present the superconductive currents, they lead to the fact that the torus ceases to be electrically neutral. From this it is possible to draw the conclusion that the vortices bear on themselves not only magnetic-flux quanta, but still electric charges.

If we change direction of flow in the primary winding, then the dependence, similar to that depicted in Fig. 24, is repeated, however, it is observed strong hysteresis. This is connected with the fact that the vortices, which penetrated into the depths of the superconductor, they are attached on the stacking faults, falling into potential wells, that also leads to hysteresis.

Thus, the results of the carried out experiments unambiguously indicate the dependence of scalar potential and magnitude of the charge from their speed, which was predicted still in the work [10] and it is experimentally confirmed in the works $[59,60]$.

The dependence of the scalar potential of the charge and its values from the speed were investigated also with the warming-up of the plasma [50]. In the experiments for the warming-up of plasma the micro-bursts with the discharge of the chemical capacitors of the great capacity through the discharger. In the discharger was used the copper wire, with the connection to which the charged capacitors it was melted and evaporated, being converted into the plasma. The diagram of experiment is shown in Fig. 26 and Fig. 27.

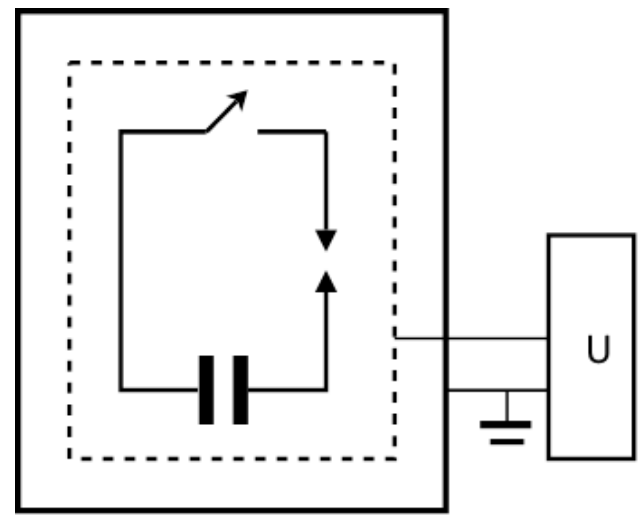

Fig. 26. Diagram of experiment with one external screen 
In the second case (Fig. 26) the oscillography was connected between the external screen and the intershield, located between the screen of the Faraday cage and the external screen.

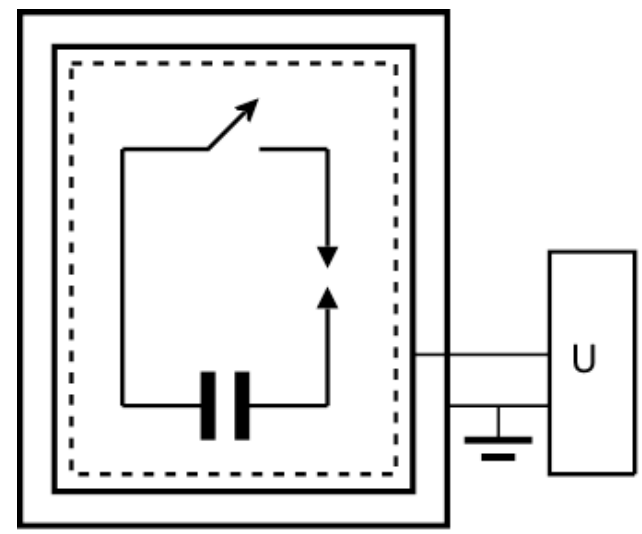

Fig. 27. Diagram of experiment with the intershield

In Faraday cell, which serves the continuous metal screen (on the figures it is depicted as dotted line) are placed the chemical capacitors of great capacity, the discharger and the key, which makes it possible to connect to the discharger the charged capacitors. The chains of outline, which include capacitor, key and discharger did not have galvanic contact with the screen of Faraday cage. Faraday cage surrounds one (Fig. 26) or two (Fig. 27) metallic of screen. Characteristic measurement of electric pulse it was achieved with the aid of the digital memory oscillography SIGLENT SDS 1072CNL.

In the first case (Fig. 26) oscillography was connected between the screen of the Faraday cage and the external screen.

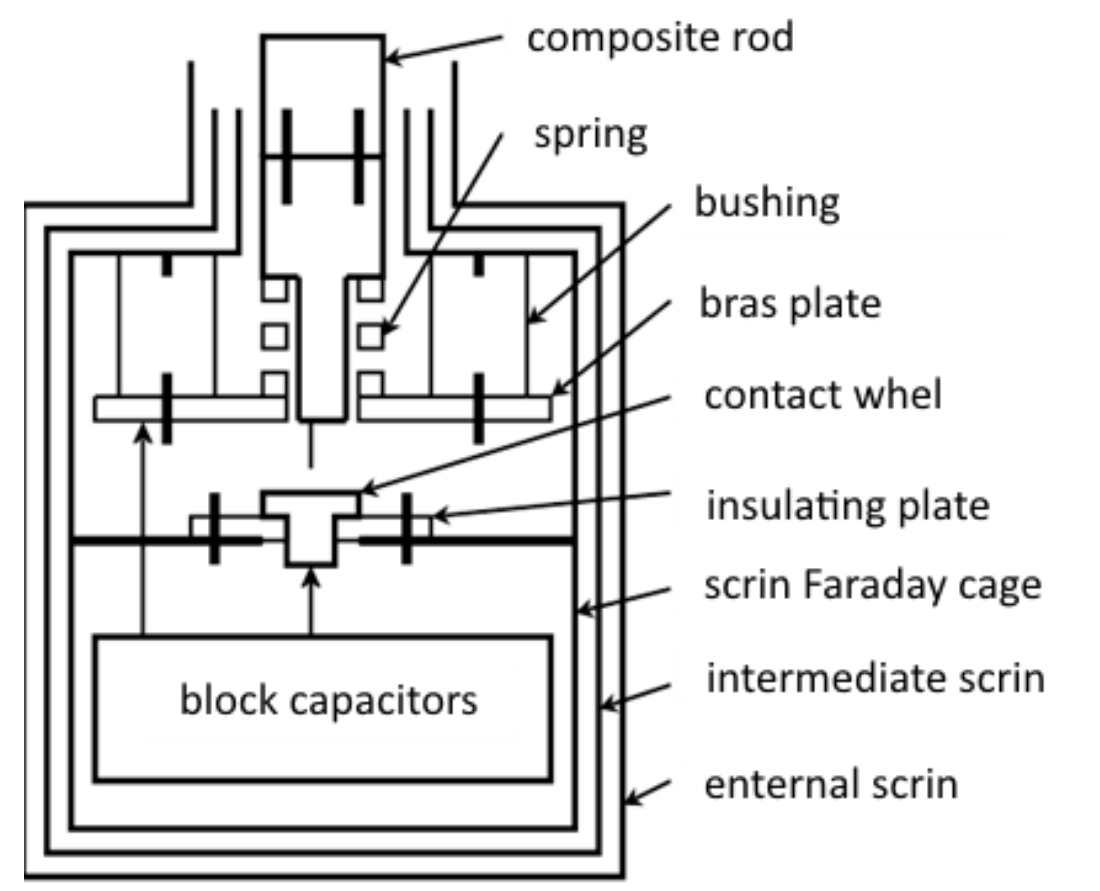

Fig. 28. The schematic of experimental installation 
The schematic of experimental installation is shown in Fig. 28.

The composite stock, which forms part of installation, consists of two parts. Its upper part is made from textolite, the lower part, made from brass, is fastened to it with the aid of the fastening pin. Between the lower part of the stock and the brass plate there is a spring, which ensures the electrical contact between the brass part of the stock and the brass plate. Inside the screen of the Faraday cage is a partition, to which is attached the insulating plate. Contact washer is located on this plate. The unit of capacitors is connected between the brass plate and the contact washer. To the lower part of the stock are attached thin copper wire, gauge $0.2 \mathrm{~mm}$, its length, which comes out from the stock $-5 \mathrm{~mm}$. During lowering of stock the wire concerns contact washer, and the charged capacitors are connected to it: wire is melted and evaporates, being converted into the plasma. In the installation they were used the collection of the chemical capacitors with a total capacity $3000 \mathrm{mi}-$ crofarad, which were charged up to the voltage $300 \mathrm{~V}$.

Fastening bolts and pins are shown in the figure by the fatty sections of lines. The joints, which make it possible to connect the oscillography between the screen of the Faraday cage and the external screen, and also between the external and intershield in the diagram are not shown. Are not shown also the joints, through which is achieved the charge of capacitor. With the measurements the cable, through which is achieved the charge of capacitor, from Faraday cage is disconnected.

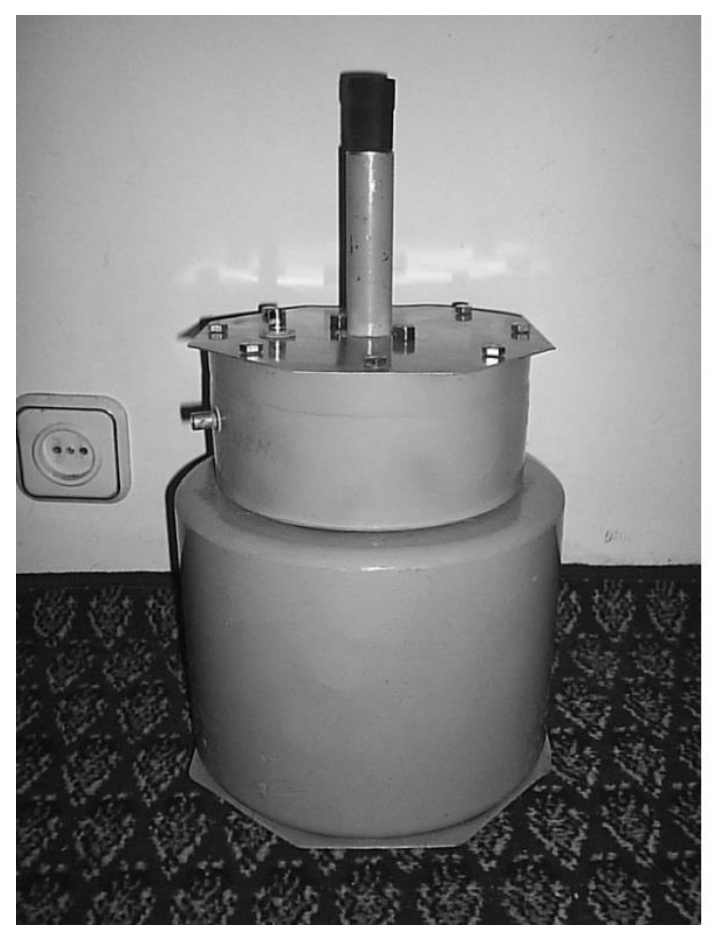

Fig. 29. The photograph of the screen of the Faraday cage

The photograph of the screen of the Faraday cage it is shown in Fig. 29.

Diameter of the upper and lower part of the screen of the Faraday cage $180 \mathrm{~mm}$ and $220 \mathrm{~mm}$ respectively. Height of the upper part $80 \mathrm{~mm}$, and lower $-220 \mathrm{~mm}$. The upper part of the screen is capped, to which is attached the tube, into which is 
put composite stock. Length of tube $100 \mathrm{~mm}$. The screen of the Faraday cage is covered with three layers of acrylic auto-enamel. This layer presents the insulator, above which stuck the aluminum foil, which presents intershield.

In Fig. 29 the separate parts of installation are depicted. The lower part of the photograph presents external screen. Its diameter $300 \mathrm{~mm}$, and a height $600 \mathrm{~mm}$. On top on the external screen, closed with cover, stands the Faraday cage. In the installation in the assembled form the Faraday cage is located inside the external screen on the insulating table.

In the process of experiments it was established that the surge voltage appears with the capacitor discharge through the discharger between the screen of the Faraday cage and the external screen.

In order to be certified in the fact that with the warming-up of plasma in Faraday cage actually is formed the unitary charge, was carried out the following experiment. It is known that with the rubbing by the fur of amber on it is formed the negative charge. By the fur of model from the amber it through the tube in the upper lid of camera was rapidly introduced after rubbing, and then just as rapidly it was withdrawn from the Faraday cage. The pulse, registered with the connection of the oscillography between the screen of the Faraday cage, it is shown in Fig. 30.

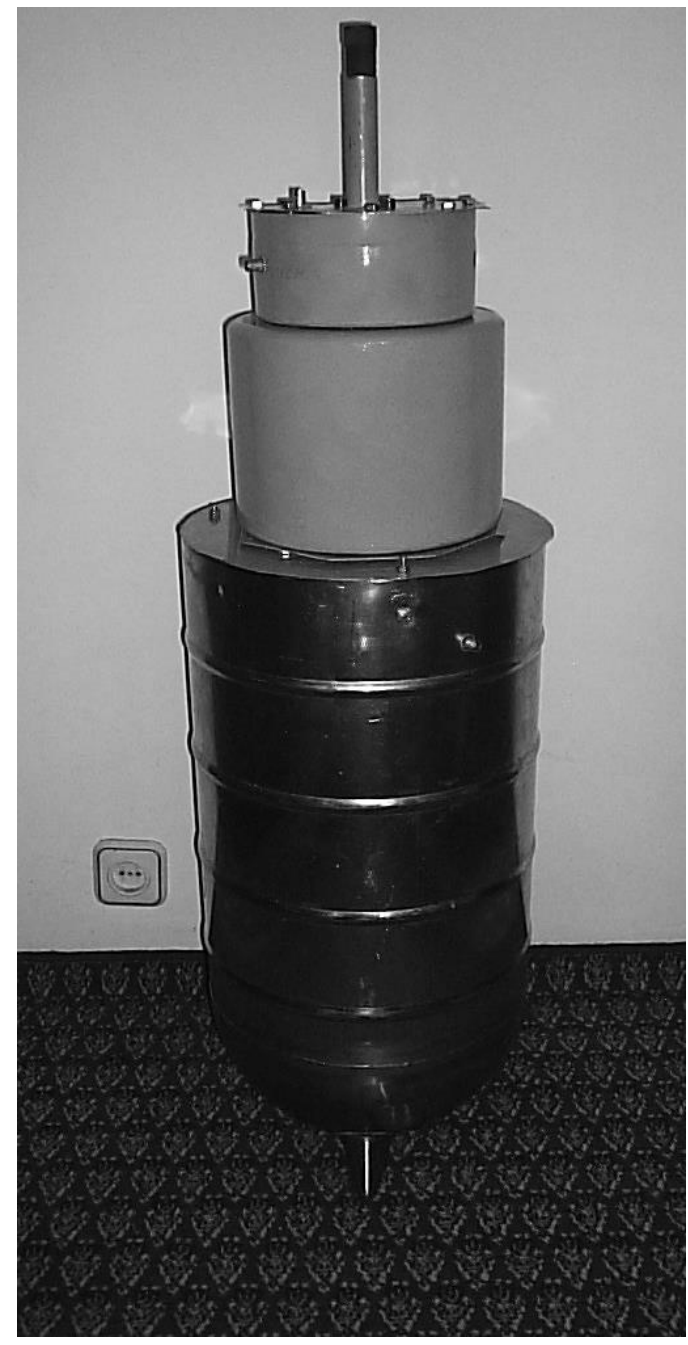

Fig. 30. Photograph is installation in the dismantled form 


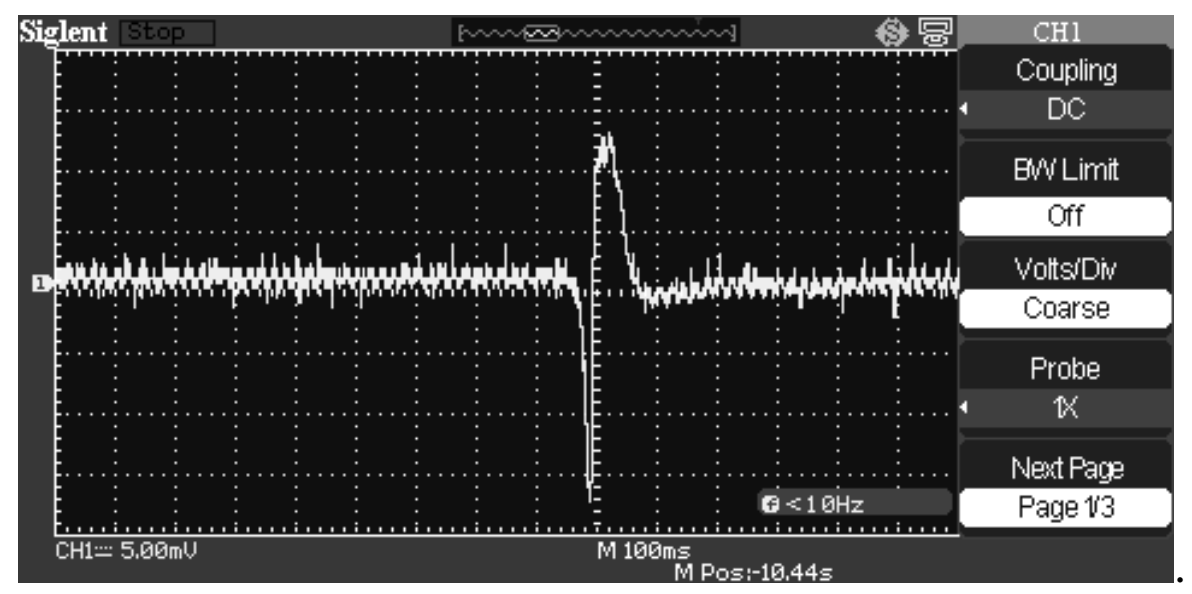

Fig. 31. Shape of pulse with the rapid withdrawal of the model of the charged amber from the Faraday cage

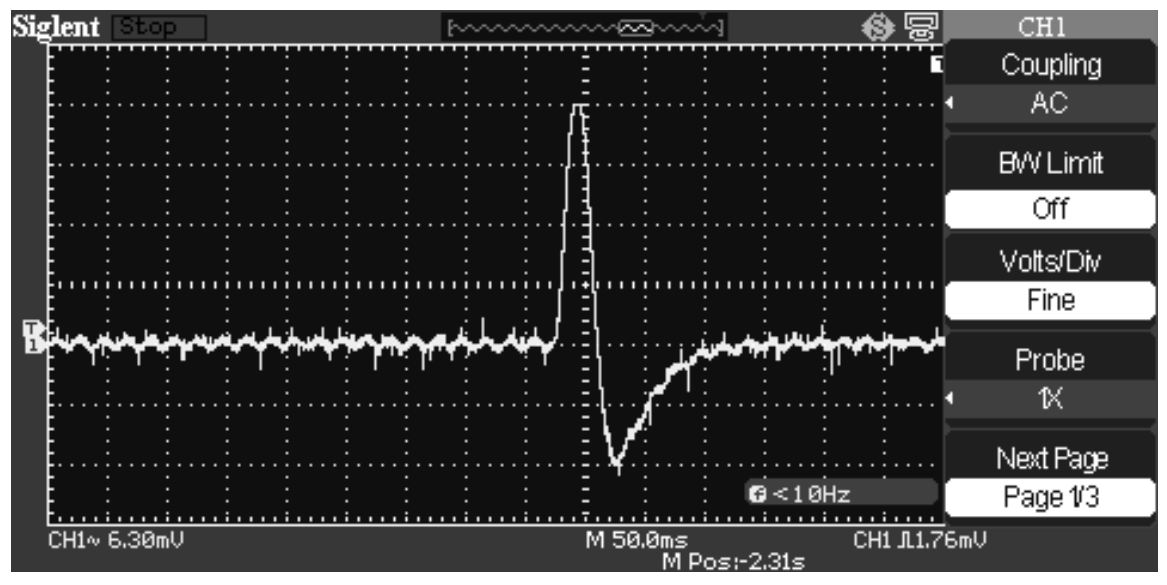

Fig. 32. Shape of pulse with the rapid withdrawal of the model of the charged amber from the Faraday cage

If we model from the amber slowly introduce into the cell, to and then rapidly withdraw it from there, then is observed the pulse, shown in Fig. 32.

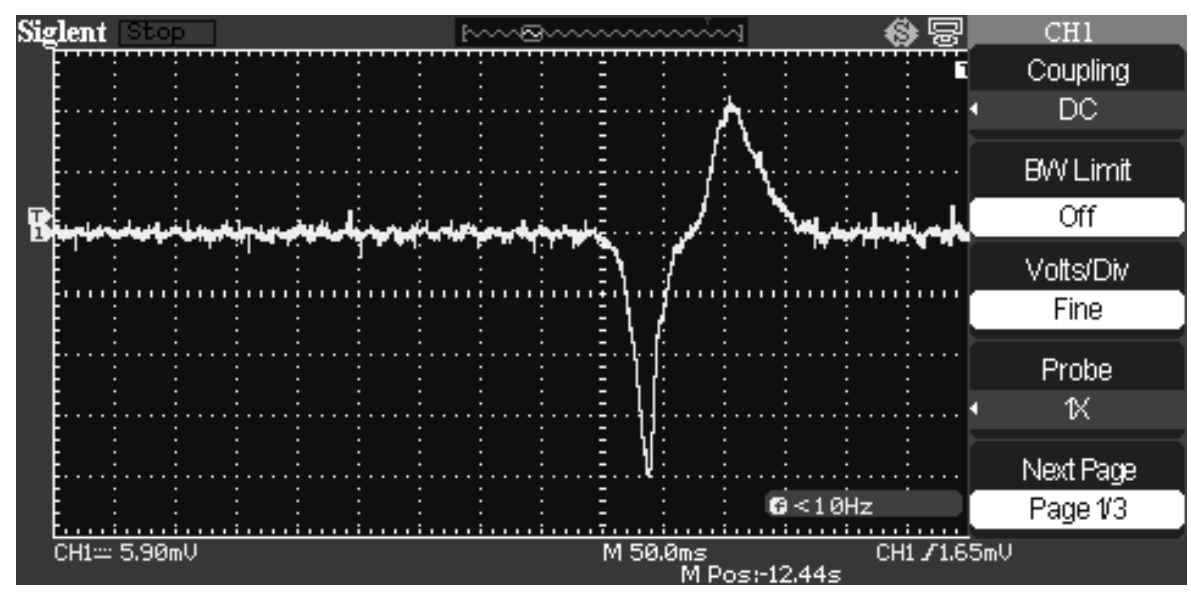

Fig. 33. Voltage pulse, obtained with the rapid introduction and the subsequent withdrawal from the Faraday cage of the charged model of amber 
If we the charged model from the amber rapidly introduce into the cell and to immediately just as rapidly withdraw it from there, then is observed pulse shown in Fig. 33. It is evident that between the negative and positive part of the pulse is a region, where the derivative of the pulse amplitude on the time decreases. This is connected with the fact that with the mechanical introduction and the withdrawal of the model of amber from the Faraday cage it is not possible to instantly change the speed of stock for the reverse.

In the following stage of studies it was explained, in what time charged the capacitors are discharged through the plasma formed in the discharger.

In Fig. 34 the oscillogram of transient process with the capacitor discharge through the discharger is represented.

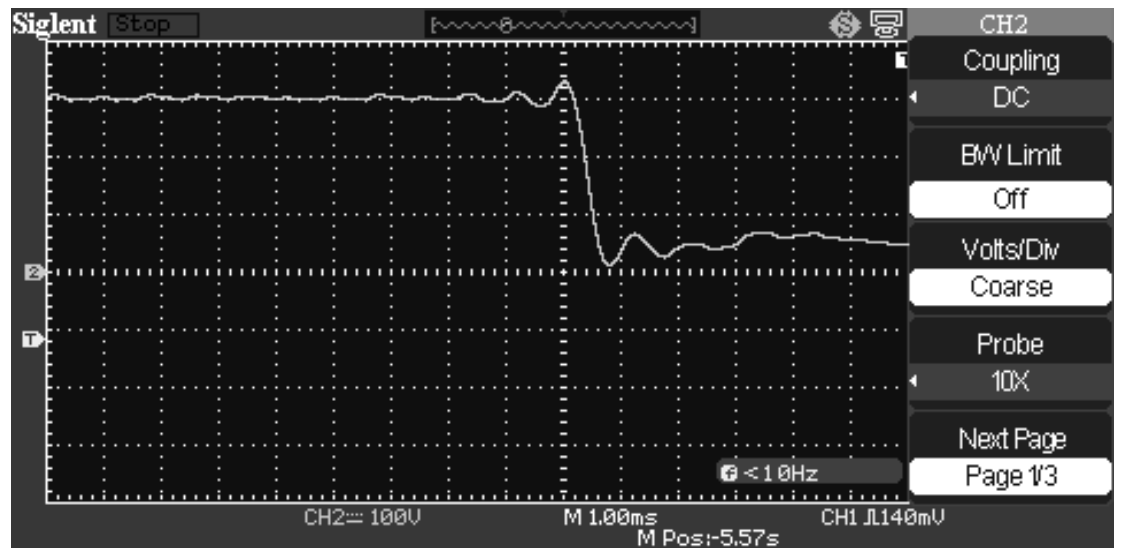

Fig. 34. The oscillogram of transient process with the capacitor discharge through the discharger is represented

It is evident that in discharge time voltage across capacitors falls with $300 \mathrm{~V}$ to $50 \mathrm{~V}$. In this case the discharge time is approximately one 600 microsecond. The difference between the energy of the capacitors, charged to $300 \mathrm{~V}$ in those charged to $50 \mathrm{~V}$ composes $162 \mathrm{~J}$; therefore the average power of micro-burst is $270 \mathrm{~kW}$. In the initial stages of the formation of the plasma, when current and voltage on the discharger are maximum this value to strands more.

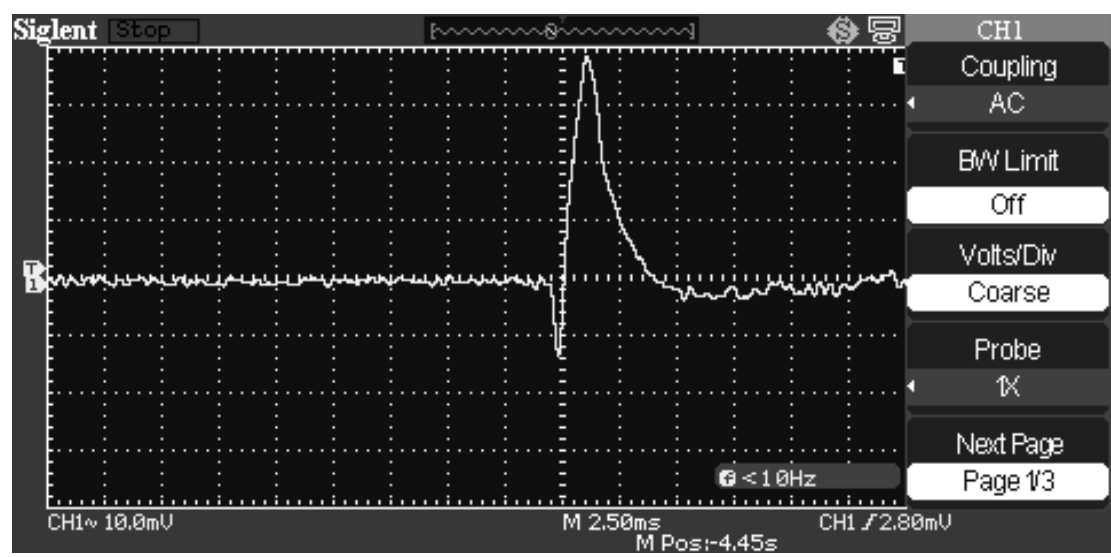

Fig. 35. Form of the voltage pulse 
The form of the voltage pulse between the external screen and the screen of the Faraday cage, obtained with the discharge through the discharger of the capacitors with a capacity 6000 microfarad, charged to the voltage $300 \mathrm{~V}$, it is shown in Fig. $35,36$.

The same pulse with the scale X-axis is $1 \mathrm{~ms}$ is shown in Fig. 36. Should be focused attention on the fact that the formation of the negative part of the pulse practically it coincides with the capacitor discharge time (Fig. 31), when through the plasma maximum current flows precisely in this time and the maximum warmingup of plasma occurs, since with the flow through it of high currents the warmingup is connected not only with its effective resistance, but also with the pinch effect.

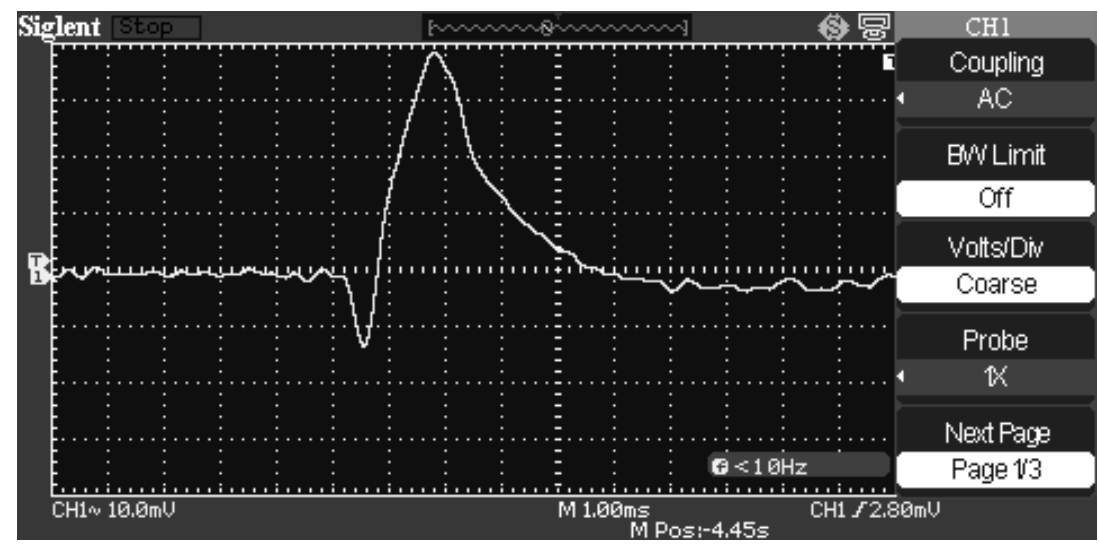

Fig. 36. Form of the voltage pulse

If we compare Fig. 31, where is shown the shape of pulse with introduction into of the Faraday cage of the charged amber and Fig. 35, that it is possible to see that the shapes of pulses it is very similar. The difference only in the fact that with the mechanical introduction and the withdrawal of amber from the cell it is not possible to ensure this pulse time and the steepness of its fronts as with the electrical discharge. in Fig. 35 the stages of warming-up and cooling of plasma are well visible, evident also that its heating occurs much faster than cooling.

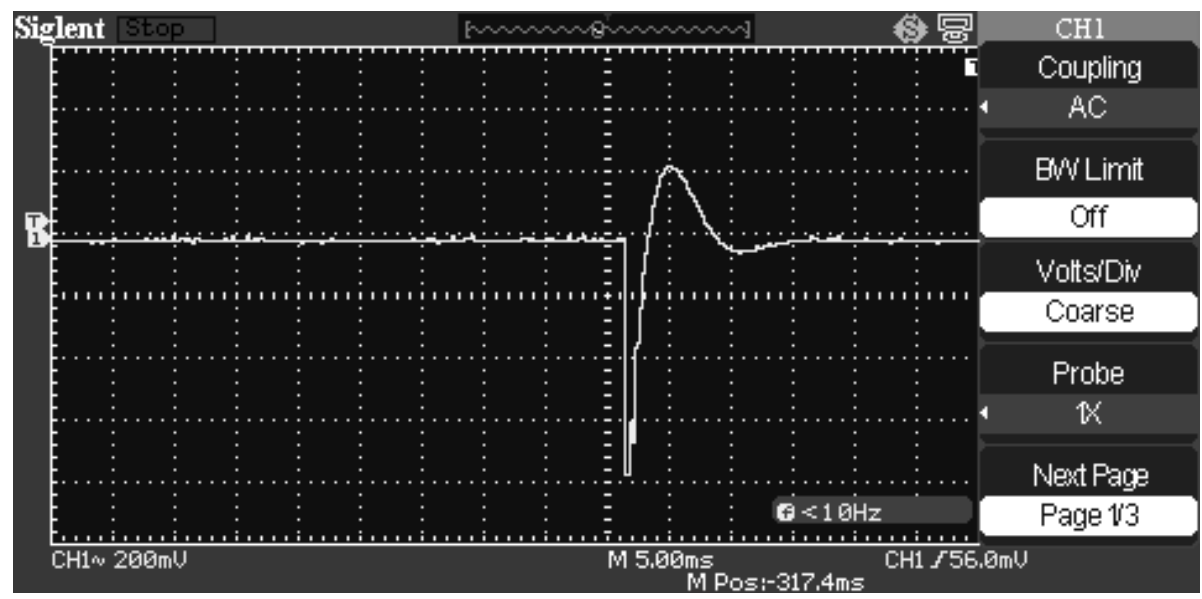

Fig. 37. Derivative of the current, which flows through the plasma 
The total capacitance of the input circuit of oscillography and capacity between the screen of the Faraday cage and the external screen is $204 \mathrm{pF}$, and the resistance of the input circuit of oscillography equally by $1 \mathrm{Mom}$, therefore, the input circuit of oscillography is differentiating. Therefore the oscillograms, depicted in Fig. 34 and Fig. 36 they present the derivative of the voltage pulse, which appears between the screen of the Faraday cage and the external screen. Naturally to assume that the temperature of plasma, since it has effective resistance, it is proportional to the current, which flows through it.

The derivative of the current, which flows through the plasma was removed with the aid of the chain, inductively connected with the conductors of the outline, along which flows the current of discharge. This dependence is represented in Fig. 36.

Comparing the pulses, represented in Fig. 34 and Fig. 36 it is possible to see that they are identical. This means that in the case in question we deal concerning the generation and the disappearance in the Faraday cage of the unitary charge, connected with the electron motion. In the formed plasma the number of electrons and positive ions is equal, but electrons have high speed, than ions.

Given experimental data are the proof of the fact that in the process of the warming-up of plasma with an equal quantity in it of electrons and positive ions in it is formed the unitary negative charge, not compensated by positive ions the experiment examined it directly confirms that the fact that the invariant of speed is only the polarity of the moving electric charge, but its absolute value depends on speed.

\section{$\S 21$. Electric pulse of nuclear and other explosions}

According to the program "Starfish" 1962 of the USA exploded in space above Pacific Ocean H-bomb. This event placed before the scientific community many questions [20,21]. It is earlier into 1957 future Nobel laureate doctor Hans Albrecht Bethe being based on the theory of dipole emission, predicted that with a similar explosion will be observed the electromagnetic pulse (EMI), the strength of field of which on the earth's surface will comprise not more than $100 \mathrm{~V} / \mathrm{m}$. Therefore entire measuring equipment, which had to record electromagnetic radiation, was disposed for registering such tensions fields on. But with the explosion of bomb discomfiture occurred, fields on the tension of electrical, beginning from the epicenter of explosion, and further for the elongation of more than $1000 \mathrm{~km}$ of it reached several ten thousand volt per meters.

There is a post form an electrical pulse (EMP), made at a distance of 1,300 km from the explosion site (Fig. 38) obtained by on «Starfish» Program tests.

From the above figure shows that the electromagnetic pulse is not only a very large amplitude, but also a very short duration $150 \mathrm{~ns}$.

Since doctor Bethe forecast did not justify, it was subsequently advanced a number of the theories, intended to explain experimental data.

The most famous was the theory in which is assumed that the formation EMI is obliged to the relativistic Compton electrons, which the rigid X-radiation knocks out from the molecules of air and which cophasal with gamma-radiation move with the relativistic speeds in the direction of propagation of electromagnetic wave. It 
assumes this model that the process of the pulse shaping is not the property of explosion itself, but is the second effect, connected X-radiation it with the fact that knocks out from the molecules of air Compton electrons. It follows that the pulse is extended from the ionosphere into the lower layers of the atmosphere, and its field higher than ionosphere, directly in space itself, they be absent from it.

But, if we with the aid of the theories examined even somehow possible explain the presence of electrical fields on in the visibility range of explosion, then the fact of strong ionospheric disturbances at large distances from the explosion, which it accompanied, to explain difficultly. Thus, after explosion in the course of several ten minutes there is no radio communication with Japan and Australia, and even at a distance into $3200 \mathrm{~km}$ from the epicenter of explosion were fixed ionospheric disturbances, which several times exceeded those, which are caused by the most powerful solar flares. Explosion influenced also the automatic spacecraft. Three satellites were immediately disabled by electromagnetic pulse. The charged particles, which were appeared as a result explosion, were seized by the magnetosphere of the Earth, as a result of which their concentration in the artificial Earth radiation belt it increased by 2-3 orders. The action of radiation belts led to the very rapid degradation of solar batteries and electronics in seven more satellites, including in the first commercial telecommunication satellite Tele-Star. On the whole explosion derived from system third of the automatic spacecraft, which were being found in low orbits at the moment of explosion.

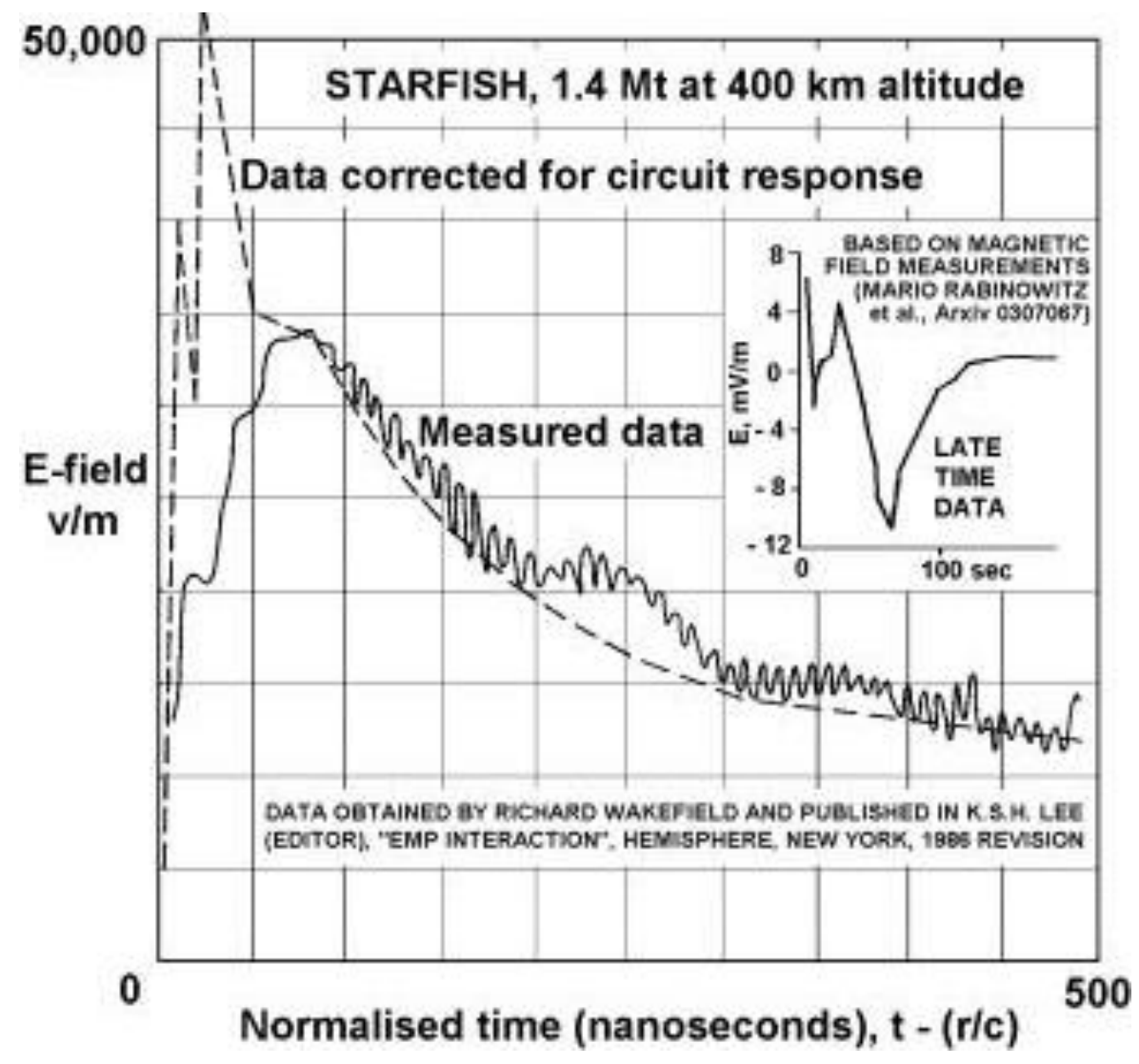

Fig. 38. Experimental dependence of the amplitude of the EMP time obtained by on «Starfish» Program tests 
With the explosion of nuclear charge according to the program "Program K", which was realized into the USSR, the radio communication and the radar installations were also blocked at a distance to $1000 \mathrm{~km}$. As a result these tests it was established that the high-altitude nuclear explosions are accompanied by the emission of the powerful pulse, which considerably exceeds in the amplitude the value of the pulse, which occurs with the surface explosions of the same power. It was discovered, that the registration of the consequences of space nuclear explosion was possible at the large (to 10 thousand kilometers) distances from the point of impact.

From the point of view of the existing concepts of classical electrodynamics Compton models cause serious questions. For example, why all Compton electrons must move cophasal with the front of gamma-radiation with the relativistic speed. In Compton electrons the velocity vector has spatial distribution, in connection with this it is not possible to obtain such short of the pulse rise, as it takes place in actuality. In the electrodynamics such mechanisms, which give the possibility to obtain the single-pole pulse of electric field without the three-dimensional separation of charges in this place theoretically be absent. But in the pulse rise time, which is calculated by tens of nanoseconds, to obtain the three-dimensional separation of charges, which will ensure the field strength obtained during the experiment, it is impossible. Compton ionization itself leaves entire system as a whole of electrically neutral. In addition to this, the ionosphere does not have sharp boundary; therefore its ionization by X-radiation will pass gradually in proportion to the advance of the wave of emission, which will lead to an increase in the duration of terminal impulse up to several milliseconds.

Is known that the problem of this phenomenon attempted together with his students to solve and academician Zeldovich [61]. However, in the existing sources there is no information about the fact that this problem was solved by it. Consequently, the everything indicates that within the framework existing classical electrodynamics the results, obtained with the tests according to the program "Starfish" of and "Program K", cannot be explained thus far.

In what does consist the danger of the forecasts, which does give the model of Compton electrons? Problem in the fact that this model excludes the possibility of the presence fields on pulse in space. The let us assume that indicated model is accurate, and, relying on it as in the past for the predictions of doctor Bethe, will be produced sequential nuclear explosion in space, which will put out of action a large quantity of satellites. Moreover this explosion can be both the planned and realized for terrorist purposes. Then be justified already is late.

Let us undertake the attempt, using a concept of scalar-vector potential, to explain obtained experimental data, and let us also show that with the explosion of nuclear charge in space, there are not fields of electromagnetic pulse (EMP), but pulse electric fields (PEF), in which the magnetic field is absent. The fields PEF in space having much more significant magnitudes, than in the atmosphere and on the earth's surface.

According to the estimations at the initial moment of thermonuclear explosion the temperature of plasmoid can reach several hundred million degrees. At such 
temperatures the electron gas is no longer degenerate and is subordinated to the distribution of Boltzmann. Let us assume that the temperature of the plasmoid at the initial moment formed with the explosion composes $\sim 10^{8} \mathrm{~K}$, and the total weight of bomb and head part of the rocket, made from metal with the average electron density of $\sim 5 \times 10^{22} 1 / \mathrm{sm}^{3}$ composes $1000 \mathrm{~kg}$. General a quantity of free electrons in the formed plasma, on the assumption that all atoms will be singly ionized with the specific weight of the metal $\sim 8 \mathrm{~g} / \mathrm{cm}^{3}$, will comprise $\sim 5 \times 10^{27}$. The most probable electron velocity at the temperature indicated let us determine from the relationship:

$$
v=\sqrt{\frac{2 k_{b} T}{m}},
$$

where $k_{5}$ - Boltzmann constant, and $m$ - mass of electron.

Now, using relationship (16.4) for enumerating the increase scalar-vector potential and taking into account only terms of the expansion $\sim \frac{v^{2}}{c^{2}}$, we obtain

$$
\Delta \varphi \cong \frac{N e k_{B} T}{4 \pi \varepsilon_{0} r m c^{2}},
$$

where $e$ - electron charge, $r$ - distance from the burst center to the observation point. We determine from the formula the tension of radial electric field, which corresponds to this increase in the potential:

$$
E=\frac{N e k_{b} T}{4 \pi \varepsilon_{0} r^{2} m c^{2}}=\frac{\Delta q}{4 \pi \varepsilon_{0} r^{2}}
$$

where

$$
\Delta q=\frac{N e k_{b} T}{m c^{2}}
$$

is an equivalent charge of explosion. By this value it is necessary to understand exceeding the charge of electron gas in comparison with its equilibrium value in the metal.

One should say that with the warming-up of plasma the ions also acquire additional speed, however, since their mass considerably more than the mass of electrons, increase in their charges can be disregarded.

In accordance with formula (21.2) the tension of radial electric field in the epicenter of explosion with the assigned above parameters will compose $\sim 7 \times 10^{5}$ $\mathrm{V} / \mathrm{m}$. Certainly, are unknown neither the precise initial of the temperature of plasmoid nor mass of bomb and launch vehicle, in which it undermine nor materials, from which are prepared these elements. Correcting these data, it is possible sufficiently simply to obtain values fields on those being approaching experimental values. With resolution of this question should be considered also the screening effect of the ionosphere. 
Let us first examine the case, when the ionosphere is absent (Fig. 39). For simplification in the task we will consider that the ideally conducting limitless plane represents by the earth's surface. The solution of allocation problem fields on for the charge, which is been located above this plane, well known [4].

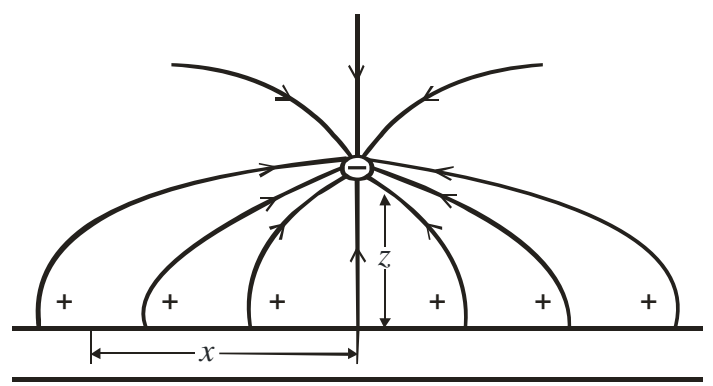

Fig. 39. Negative charge above the limitless conducting plane

The horizontal component of electric field on the surface of this plane is equal to zero, and normal component is equal:

$$
E_{\perp}=\frac{1}{2 \pi \varepsilon_{0}} \frac{z q}{\left(z^{2}+x^{2}\right)^{\frac{3}{2}}},
$$

where $q$-magnitude of the charge, $z$ - shortest distance from the charge to the plane, $x$ - distance against the observation points to the point of intersection of vertical line, lowered from the point, where is located charge, to plane itself.

Lower than conducting plane electric fields be absent. This configuration fields on connected with the fact that charge, which is been located above the conducting plane, it induces in it such surface density of charges, which completely compensates horizontal and vertical component of the electric field of charge in the conducting plane and lower than it. The dependence of the area charge from the coordinate of $x$ can be determined from the relationship:

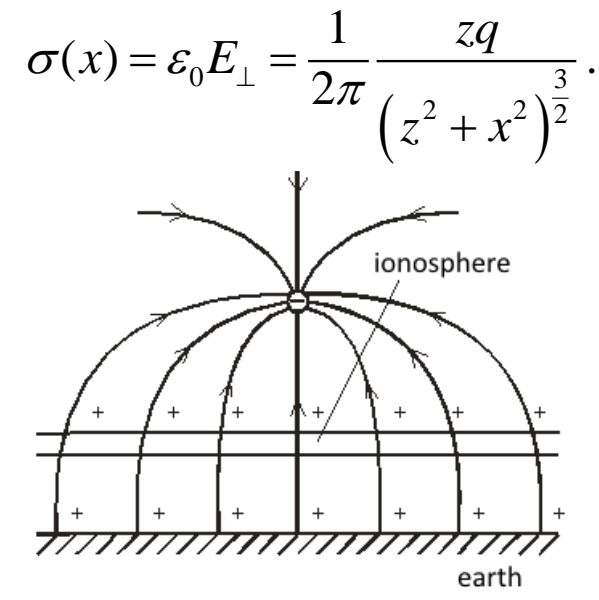

Fig. 40. Negative charge above the earth's surface with the presence of the Ionosphere

If we integrate $\sigma(x)$ with respect to the coordinate $x$, then we will obtain magnitude of the charge, which is been located above the conducting plane. In such a 
way as not to pass the electric fields of the charge $q$ through the conducting plane, in it must be contained a quantity of free charges, which give summary charge not less than the charge $q$. Let us examine from these positions the screening effect of the ionosphere (Fig. 39).

If charge will appear at the indicated in the figure point, thus it will gather under itself the existing in the ionosphere free charges of opposite sign for compensating those fields on, which it creates in it. However, if a quantity of free positive charges in the ionosphere will be less than first, which is necessary for the complete compensation for the equivalent charge of explosion, then its fields will penetrate through the ionosphere. In this case the penetrated fields, in view of the screening effect of the ionosphere, can be less than the field above it. Entire this picture can be described only qualitatively, because are accurately known neither thickness of the ionosphere nor degree of its ionization on the height.

The sphericity of the ionosphere also superimposes its special features on the process of the appearance of the compensating surface charges. This process is depicted in Fig. 41.

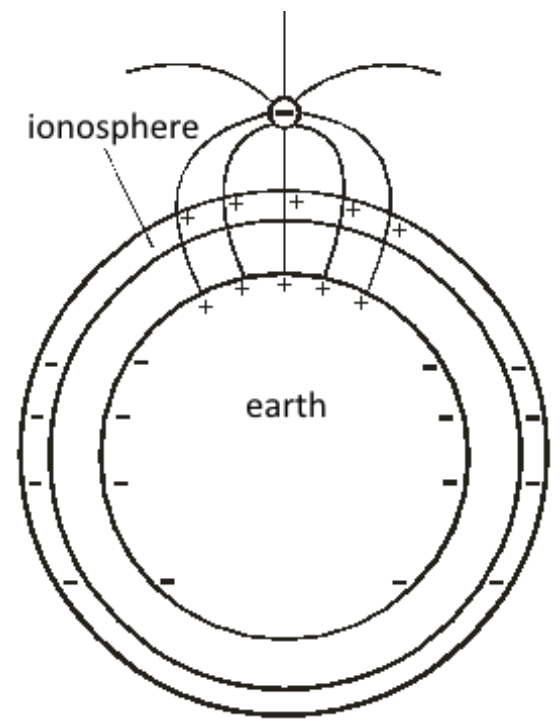

Fig. 41. Negative charge above the earth's surface with the presence of the ionosphere

The tendency of the emergent charge to gather under itself the compensating charges will lead to the longitudinal polarization of the substantial part of the ionosphere. The compensating positive charges will be located in the ionosphere directly in the straight visibility under the charge and here them it will be in the surplus, while beyond the line-of-sight ranges in the surplus they will be negative charges. And entire system charge - the ionosphere - the earth will obtain additional dipole moment. The distribution of induced charge in the ionosphere will depend on the height, at which is located the charge, and also from the position of the sun with respect to the charge, since. the degree of ionization of the ionosphere depends on its position.

With the nuclear explosion is synchronous with the electrical radial fields, which are moved from the plasmoid with the speed of light, moves the front of X- 
radiation. This emission will ionize the atmosphere, increasing its conductivity, while this will, in turn, increase the shielding functions of the atmosphere from the penetration into it of the pulses of the subsequent explosions, if such arise. Furthermore, since the negative potential of plasmoid at the initial moment of the explosion of very large, from the cluster will be temporarily rejected some quantity of electrons, which also after a certain time will fall into the ionosphere. The partial neutralization of the electrons, which fell into the ionosphere, will occur, when the positive ions of plasmoid will also reach the ionosphere. But this will concern only those ions, the radial component of speed of which was directed to the side of the ionosphere. The same electrons and ions, whose radial component was directed to the side from it, will leave the limits of the earth's gravity and they will present the similarity of that solar wind, which is the consequence of the evaporation of the solar corona or flashes on the solar surface. Those complex processes, which accompany nuclear explosion, now are only schematically outlined, and is in prospect still extensive work, on the recreation of these processes for the actual conditions. It is obvious that to make this is possible only numerical methods.

The model examined speaks, that nuclear explosion will lead not only to the appearance PEF in the zone of straight visibility, but also to the global ionospheric disturbance. It is known that the explosions according to the program "Starfish" and according to the program "Program K" led to the presence of large interferences with radio-technical and radar systems at large distances from the epicenter of explosion. Certainly, the electric fields in space, generated by this explosion, have very high values and present the major threat for the automatic spacecraft.

Now let us return to the horizontal component of electrical fields on the earth's surface, generated with the explosion. It is understandable that these fields represent the tangential component of radial fields on, that go from the point of explosion. Specifically, these fields cause the compensating currents, which create the compensating surface charges. It is possible to calculate the order of the summed currents, which will have radial directivity with respect to the epicenter of explosion. For this let us calculate summary compensating grain surface on the earth's surface, which must be formed with the explosion of nuclear charge. This charge is equal to the charge of plasmoid with the opposite sign

$$
q=4 \pi \varepsilon_{0} r^{2} E .
$$

After conducting calculations according to this formula, on the basis of the actually measured vertical tensions of electrical fields on in the epicenter of explosion $\left(5.2 \times 10^{4} \mathrm{~V} / \mathrm{m}\right)$, with the distance to the explosion $400 \mathrm{~km}$ of we obtain the charge $\sim 10^{6}$ coulomb. However, the value of charge, calculated according to formula (20.3) they will compose $\sim 1.2 \times 10^{7}$ coulomb. This divergence, as it is already said, can be connected with the screening effect of the ionosphere. If the building-up of electric field it is $\sim 150 \mathrm{~ns}$, then the summed current, directed toward the epicenter of explosion, must compose $\sim 3 \times 10^{11} \mathrm{~A}$. Certainly, this number is somewhat overstated, because the compensating charges are attracted not to one point, which is been the epicenter of explosion, but to the sufficiently extensive region in its environment. But even if this value decreased several orders, previous the strength of compensating currents will be very large. It is now understandable, why on Oahu 
island, that is been located at a distance $1300 \mathrm{~km}$ of from the epicenter of explosion, burnt 300 street lamps, and near Dzheskazgan in the air telephone line with the extent $570 \mathrm{~km}$ of arose the currents $\sim 2.5 \mathrm{kA}$, which burnt in it all safety fuses. Even to the power cable by extent is more than $1000 \mathrm{~km}$, which connects AlmaAta and Akmola, and the having armored screen from lead, braiding from the steel tape, and located on the depth $0.8 \mathrm{~m}$, such focusing arose, that operated the automata, after opening from the cable power station. Certainly, the pulse of tangential currents, although the less significant than on the earth's surface, will be also in the ionosphere, which will lead to its disturbance on global scales.

Entire process of formation PEF with the explosion of charge in space can be described as follows. At the moment of explosion in the time of the detonation of nuclear charge, which lasts several nanoseconds, is formed dense plasmoid with the temperature in several ten and even hundreds of millions of degrees. This cluster generates the powerful gamma emission, which is extended in different directions from the cluster with the speed of light. Simultaneously is generated the radial electric field, which also is extended in the radial direction from the cluster with the speed of light. Radial electric fields PEF and gamma-radiation reach the ionosphere simultaneously. During its further motion to the side of the earth's surface, if explosive force for this it is sufficient, $\mathrm{X}$-radiation begins to ionize and the layers of the atmosphere, which are been located lower than the ionosphere. The process of the ionization of upper air and the penetrations in them of radial electric field will simultaneously occur. In the ionized layers due to the presence of radial electric field will arise the radial currents, which will lead to the stratification of charges and to the vertical polarization of conducting layers. The processes of the polarization of the atmosphere will last as much time, as will exist radial field, and also conductivity of ionized air. Since the ionosphere will not be able to ensure the charge, necessary for the complete compensation for the radial field of plasmoid, these fields, although in the weakened form, they will continue to be extended in the direction of the earth's surface. Reach its, electric fields will create powerful radial currents. The process of propagation of X-radiation and radial fields on through the ionosphere it will lead to its additional ionization and polarization, and also to the appearance of a pulse of tangential currents. The pulse of tangential currents in the ionosphere will apply to distances considerably greater than the visibility range of explosion, which will lead to the global ionospheric disturbances.

Up to that moment, when the flow of rigid gamma emission and ionization of atmosphere cease, the part of the atmosphere, ionized lower than the existing boundary of the ionosphere, will cease to be conductor, and is, therefore, the threedimensional divided charges will prove to be closed in it. The electrons closed in the atmosphere will as before create some static potential difference, which will slowly relax to the extent of the presence of the residual conductivity of the atmosphere. It should be noted that the polarity of this field will be opposite to the polarity of initial PEF that also is observed in actuality. This means that the radial electric field, observed on the earth's surface, will be first directed from the earth toward the epicenter of explosion, but at some moment of time it will change its polarity. 
Becomes clear and that, why after space nuclear explosion an even longer time is observed the residual glow of the atmosphere under the point of impact. This glow is obliged to those electrons, which during the first stage development PEF were displaced of the ionosphere into the denser layers of the atmosphere, and then, after the termination of the ionizing effect of gamma emission, they remained closed in the little conducting atmosphere, continuing to ionize it.

Thus, the appearance PEF with the nuclear explosion are the properties of explosion itself, but not second phenomena. Its properties and characteristics can be explained within the framework to the concept of scalar- vector potential. Studying topology PEF on the earth's surface, it is possible to judge also the subsequent processes of polarization and depolarization of the ionosphere, atmosphere and earth's surface. With the explosion in the atmosphere very process of formation PEF and its development are connected with the presence of the atmosphere, and also by the presence of conductivity on the earth's surface and this will also superimpose its special features on shaping fields on PEF. With the explosions in immediate proximity from the earth's surface the equivalent charge of the cloud of explosion will see its mirror reflection under the earth's surface, forming the electric dipole. For this reason the region of propagation fields on PEF it will be strongly reduced, since the fields of dipole diminish according to the cubic law.

Now should be made one observation apropos of term itself the electromagnetic pulse EMI, utilized in the literary sources. From this name should be excluded the word magnetic, since. this process presents the propagation only of radial electrical fields on, and in this case magnetic fields be absent. It is another matter that electric fields can direct currents in the conducting environments, and these currents will generate magnetic fields, but this already second phenomenon.

Would seem, everything very well converges, however, there is one basic problem, which is not thus far examined, it concerns energy balance with the explosion. If we consider that one ton of trotyl is equivalent $4.6 \times 10^{9} \mathrm{~J}$, then with the explosion of bomb with the TNT equivalent $1,4 \mathrm{Mt}$. are separated $6.44 \times 10^{15} \mathrm{~J}$. If we consider that the time of detonation is equal to $150 \mathrm{~ns}$, then explosive force composes $\sim 1.3 \times 10^{23} \mathrm{~W}$. Let us say for an example that the power of the radiation of the Sun $\sim 3.9 \times 10^{26} \mathrm{~W}$. Let us examine a question, where how, in so short a time, can be the intake, isolated with this explosion.

In accordance with Stephan equation Boltzmann the power, radiated by the heated surface, is proportional to the fourth degree of its temperature:

$$
P=\sigma s T^{4}
$$

where $\sigma=5.67 \cdot 10^{-8} \frac{B m}{M^{2} K^{4}}$ - Stefan-Boltzmann constant, and $s$ - area of radiating surface.

If we take the initial temperature of the plasmoid $\sim 10^{8} \mathrm{~K}$, then with its initial diameter $1 \mathrm{~m}$ of (in this case the surface area of cluster it is $\sim 3 \mathrm{~m}^{2}$ ) entire explosive energy will be radiated in the time $\sim 0.4 \mathrm{~ns}$. But if we take the initial temperature $\sim 10^{7}$, then this time will be already $\sim 400 \mathrm{~ns}$. Thus, one should assume that the initial temperature of plasmoid to be located somewhere between the undertaken val- 
ues. Wavelength, on which will be radiated a maximum quantity of energy, is determined by the Wiens law

$$
\lambda_{\max }=\frac{0,28975}{T} \frac{\mathrm{sm}}{K} .
$$

If we substitute here the value of the temperature $5 \times 10^{7} \mathrm{~K}$, then we will obtain the wavelength on the order $6 \AA$, which corresponds to rigid X-radiation. Its temperature will begin to fall in proportion to cooling cluster and $\lambda_{\text {макс }}$ will begin to be shifted into the visible part of the spectrum.

But the mechanism of losses examined is not only. Since with the temperature of cluster are unambiguously connected its electric fields, immediately after detonation they will be maximum, and then with a temperature drop of cluster they will begin to decrease proportional to temperature. However, the energy, necessary for their creation, will fall not as rapidly as energy necessary for creating the $\mathrm{X}$ radiation.

Appears one additional important question about which a quantity of electrons it will leave plasmoid. In order to answer it, let us examine the condition of the electro neutrality of plasma. At that moment when metal is converted into the plasma, occurs not only the passage of substance from one state of aggregation to another, but also changes the statistics of the description of electron gas. In the solid state statistician Fermi-Dirac's this, while in the state of plasma - statistician Boltzmann's this. When electron gas was located in the steadfast conductor, then in the state of electro neutrality to each ion it was fallen along one free electron. Let us determine from the point of view of the concept of scalar- vector potential, what relationship must be observed between the electrons and the ions in the plasma so that it would also remain electrically neutral. Before solid became plasma, the electron density and ions was identical and, therefore, the absolute values of their charges were equal, i.e.,

$$
e N_{e}=e N_{n p} \text {. }
$$

After the transformation of substance into the plasma general equivalent electron charge increased, to the value, determined by relationship (20.3), and in ions it remained practically before. Now already for observing the electro neutrality must be observed the relationship:

$$
N_{e(n n)}\left(1+\frac{k_{5} T}{m_{e} c^{2}}\right)=N_{n p},
$$

where $N_{e(n \pi)}$ - equilibrium quantity of electrons in the plasma.

Is evident that this equilibrium quantity is less than to the passage of substance into the state of plasma. Difference composes

$$
\Delta N=N_{n p}\left(1-\frac{1}{1+\frac{k_{b} T}{m_{e} c^{2}}}\right) .
$$

For example, at a temperature $\sim 10^{8}$ the value, which stands in the brackets, will compose approximately 0.13 . This means that at the temperature indicated, for re- 
taining the electro neutrality of plasma, $13 \%$ of a total initial quantity of electrons had to it leave. We will call this effect the effect of temporarily excess electrons. Word "is temporarily" used by in the sense that temporary they appear as long as plasma is hot. In this connection clear to become that, from where, for example, on the surface of the sun appear powerful magnetic fields, especially when at it appear spots. These fields are induced by those currents, which overflow between the regions of plasma, which have a different temperature.

We in sufficient detail examined the behavior of the static charge above the conducting plane. But in actuality there is not a static charge, but a charge, which lives only several hundred nanoseconds. If in the origin of coordinates is located the charge $Q(t)$, depending on time, then the electric fields, created by it in the surrounding space, can be found from the relationship

$$
\varphi(r, t)=\frac{Q(t)\left(t-\frac{r}{c}\right)}{4 \pi \varepsilon r}
$$

which correspond the being late longitudinal electric fields

$$
E(r, t)=\frac{Q(t)\left(t-\frac{r}{c}\right)}{4 \pi \varepsilon r^{2}} .
$$

In accordance with relationships (21.6), (21.7) the short-lived charge generates so short-term a pulse of longitudinal electrical fields on, which in the space are extended with the speed of light and is formed the spherical layer, whose thickness is equal to the lifetime of charge, multiplied by the speed of light. If we consider that for our case the time of life of charge composes the half-width of pulse PEF (somewhere about $100 \mathrm{~ns}$ ), then the thickness of this layer will be about $30 \mathrm{~m}$. Spherical layer, reach first of the ionosphere, the earth will and then direct there the same radial currents, as if static charge appeared and, after existing $100 \mathrm{~ns}$, it disappeared.

As was already said, analyzing the topology of pulse PEF, it is possible to judge about the temperature of plasma and the processes of proceeding in it. This method can be used also for diagnostics of other forms of plasma. For plasma itself there is no difference whatever in by what form of its energy they heat up, is important only quantity of free electrons, i.e., the degree of ionization, which depends on the final temperature of plasma. Laser warming-up is considered as the promising method of its warming-up for realizing of thermonuclear fusion. In this case the samples under investigation undergo the action of powerful laser pulse. Model in short time is converted into the high-temperature plasma, i.e., there is a certain similarity of the behavior of plasma with the nuclear explosion. For these purposes it suffices to surround sample under investigation by two conducting screens and to connect between them high-speed to oscillography with the high input resistance. External screen in this case should be grounded. At the moment of the warming-up of plasma will arise PEF. Moreover a potential difference between the screens will arise much earlier than the material particles of plasma they will reach the walls of the first screen. Studying the topology of the recorded pulse, it is possible to judge the temporary energy processes of the warming-up of plasma. It is not difficult to 
calculate the expected potential difference between the screens depending on the temperature and quantities of free charge carriers in the heat plasma. After using relationships (21.5) and (21.7), for the case, when $k_{B} T \ll m c^{2}$ we obtain:

$$
U=\frac{N e k_{b} T}{4 \pi \varepsilon_{0} m c^{2}}\left(\frac{1}{r_{1}^{2}}-\frac{1}{r_{2}^{2}}\right)
$$

where $r_{1}, r_{2}$ - radius of external and internal screens respectively, and $N$ - quantity of free electrons in the heated plasma.

The fact of the presence of excess electrons should be considered, also, with realizing of controlled thermonuclear fusion, since this phenomenon must influence also the stability of plasma with its warming-up.

\section{§ 22. Theoretical bases of giperkontinual physics and giperkontinual electrodynamics}

According to Coulomb's law, the module $F$ of the force $\mathbf{F}$ of interaction of two fixed (fixed) point charges $q_{0}, q$ in the vacuum, directed along the straight line, which connects these charges, is proportional to their absolute values $\left|q_{0}\right|,|q|$ and it is inversely proportional to the square of the distance $r$ between them:

$$
F=\frac{\left|q_{0} q\right|}{4 \pi \varepsilon_{0} r^{2}}=4 \pi \varepsilon_{0} r^{2} E_{0} E,
$$

where $E_{0}, E$ - the modules of the tension of the electric field of charge $q_{0}$ at the point of the presence of charge $q$ and tension of the electric field of charge $q$ at the point of the presence of charge $q_{0}$ respectively.

If we remove the fastening of charges $q_{0}, q$, that they will arrive in the motion with the appropriate initial accelerations $\mathbf{a}_{0}=\mathbf{F} / m_{0}, \mathbf{a}=\mathbf{F} / m$, directed along the straight line, there that connecting. The moduli of these accelerations are equal

$$
a_{0}=\frac{F}{m_{0}}=\frac{4 \pi \varepsilon_{0} r^{2} E_{0} E}{m_{0}} ; a=\frac{F}{m}=\frac{4 \pi \varepsilon_{0} r^{2} E_{0} E}{m},
$$

where $m_{0}, m$ - the mass of charges $q_{0}$ and $q$.

If we pass in IRS, which moves perpendicularly to the straight line, which connects charges, with speed $\mathbf{v}$ with the module $v$, that in this IRS transverse component the tensions electrical fields on charges they will increase, by virtue of which the modules of the tension of the electric field of charge $q_{0}$ at the point of the presence of charge $q$ and tension of the electric field of charge $q$ at the point of the presence of charge $q_{0}$ they will be in this IRS equal no longer $E_{0}, E$, and respectively 


$$
E_{\perp 0}=E_{0} \operatorname{ch} \frac{v}{c} ; E_{\perp}=E \operatorname{ch} \frac{v}{c} .
$$

As a result, the module of the force of interaction of charges in this IRS also will grow and it will be equal no longer $F$, and to the value

$$
F_{\perp}=4 \pi \varepsilon_{0} r^{2} E_{\perp 0} E_{\perp}=F \operatorname{ch}^{2} \frac{v}{c}
$$

Since, according to the law of relativity, the initial accelerations of charges upon transfer into another IRS do not change, we have:

$$
a_{0}=\frac{F}{m_{0}}=\frac{F_{\perp}}{m_{0} \operatorname{ch}^{2} \frac{v}{c}} ; a=\frac{F}{m}=\frac{F_{\perp}}{m \operatorname{ch}^{2} \frac{v}{c}} .
$$

Values in the denominators

$$
m_{\perp 0}=m_{0} \operatorname{ch}^{2} \frac{v}{c}, m_{\perp}=m \operatorname{ch}^{2} \frac{v}{c}
$$

let us name the transverse masses of charges $q_{0}, q$, and usual masses $m_{0}, m$ it is possible to still otherwise name their longitudinal masses.

Thus, in contrast to the independent of the speed longitudinal mass, transverse mass depends on speed. Analogous situation occurs, also, with respect to the tension of the electric field of charge, namely, in contrast to independent of the speed longitudinal component of tension, transverse component depends on speed. In this case the equality is executed:

$$
\frac{m_{\perp}}{m}=\left(\frac{E_{\perp}}{E}\right)^{2}=\operatorname{ch}^{2} \frac{v}{c} .
$$

In the state of longitudinal rest $(v=0)$ transverse mass is equal to longitudinal, which makes it possible to interpret longitudinal mass as the transverse mass of longitudinal rest or simply rest mass.

The dependence of transverse mass on the speed it is natural to associate with the analogous dependence on the speed of the new form of rest energy, which let us name transverse energy of the transverse rest:

$$
W_{\perp}=m_{\perp} c^{2} .
$$

In contrast to the rest energy independent of the speed

$$
W=m c^{2} \text {, }
$$

which can be still otherwise named longitudinal rest energy, transverse energy of transverse rest on speed depends, moreover by the dependence of the same form as the transverse mass:

$$
W_{\perp}=W \operatorname{ch}^{2} \frac{v}{c} .
$$

It is analogous with situation with the longitudinal and transverse masses, in the state of longitudinal rest $(v=0)$ transverse energy of transverse rest is equal to longitudinal rest energy, which makes it possible to interpret longitudinal rest energy as transverse energy of transverse and longitudinal rest. 
The presence of mass $m$ and speed $\mathbf{v}$ causes existence of two forms of the energy: the kinetic energy

$$
T=\frac{m v^{2}}{2},
$$

which can be still otherwise named longitudinal kinetic energy, and the energy

$$
T_{\perp}=W_{\perp}-W=\left(m_{\perp}-m\right) c^{2},
$$

which can be still otherwise named transverse kinetic energy. The latter, in turn, causes existence of additional with respect to the longitudinal mass $m$ component of transverse mass $m_{\perp}$, which let us name the kinetic mass:

$$
\tilde{m}=m_{\perp}-m .
$$

Transverse kinetic energy easily is expressed as the kinetic mass:

$$
T_{\perp}=\tilde{m} c^{2} .
$$

Using dependence of transverse energy of transverse rest on the speed, let us write down equality for the transverse kinetic energy in the form:

$$
T_{\perp}=W_{\perp}-W=W\left(\operatorname{ch}^{2} \frac{v}{c}-1\right) .
$$

It is well known that in order to accelerate the material bodies have to expend energy, which is necessary to make them effective. My work is converted into kinetic energy of motion. When braking, the body gives this energy surrounding bodies, which require strength, return to that body to accelerate. This is the phenomenon of inertia. In the special theory of relativity (STR) electrical field charges depend on the speed, and it would seem, this theory was to give an answer to this interesting question. But in STR charge is an invariant speed. Although his field changes during acceleration, but this change occurs so that the increase of the field component normal to the direction of movement is compensated by the decrease of the longitudinal component. As a result, the electric field flux through a surface surrounding the charge does not depend on charge motion and remains constant.

However, within the concept of scalar charge capacity depending on the speed of the normal component of the electric field is increased, while the longitudinal remains unchanged. This approach makes it possible to explain the phenomena of inertia and kinetic energy. All material bodies consist of charged elementary particles, so the phenomena of inertia and kinetic energy of the body can be reduced to the same particle phenomena. In view of the identity of the consideration for any charged particle, the particle will be considered an electron without loss of generality.

Specific electric field energy composes

$$
w=\frac{1}{2} \varepsilon E^{2}
$$

it is expressed as the strength of this field

$$
E=\frac{e}{4 \pi \varepsilon_{0} r^{2}} .
$$


Using of the element the volume $4 \pi r^{2} d r$, we obtain of energy of field on of that being resting of the electron $(v=0)$ :

$$
W=\int_{a}^{\infty} \frac{e^{2} d r}{8 \pi \varepsilon_{0} r^{2}}=\frac{e^{2}}{8 \pi \varepsilon_{0} a},
$$

where $e$ - the charge of the electron, $\mathbf{v}$ - it's a radius.

If electron moves with speed $\mathbf{v}$, that normal component its electric field increases:

$$
E_{\perp}=E \operatorname{ch} \frac{v}{c} \approx E\left(1+\frac{1}{2} \frac{v^{2}}{c^{2}}\right) .
$$

We write the electric field normal to the direction of motion in the coordinate system shown in Fig. 42.

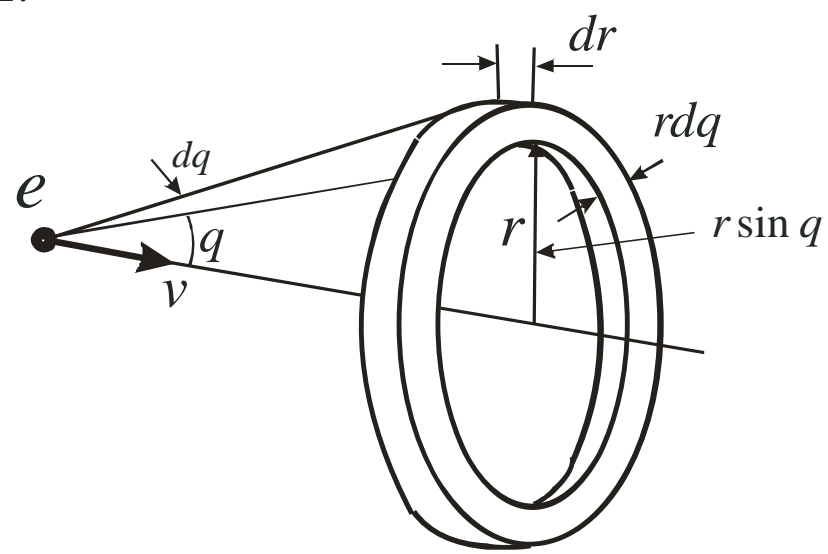

Fig. 42. The element of the volume $2 \pi r^{2} \sin q d q d r$, utilized for the calculation of energy of fields of that moving electron

$$
E_{\perp}=E\left(1+\frac{1}{2} \frac{v^{2}}{c^{2}}\right) \sin q .
$$

Then energy of fields on of that moving electron it will be written down

$$
W_{v}=\left(1+\frac{1}{2} \frac{v^{2}}{c^{2}}\right)^{2} \int \frac{e^{2} \sin ^{3} q d q d r}{8 \pi \varepsilon_{0} r^{2}} .
$$

The integration to the angle gives

$$
\int_{0}^{\pi} \sin ^{3} q d q=-\int_{0}^{\pi}\left(1-\cos ^{2} q\right) d(\cos q)=-\cos q+\frac{\cos ^{3} q}{3}=\frac{4}{3},
$$

therefore we have:

$$
W_{v}=\frac{4}{3}\left(1+\frac{1}{2} \frac{v^{2}}{c^{2}}\right)^{2} \int_{a}^{\infty} \frac{e^{2} d r}{8 \pi \varepsilon_{0} r^{2}}=\frac{4}{3}\left(1+\frac{v^{2}}{c^{2}}+\frac{1}{4} \frac{v^{4}}{c^{4}}\right) \frac{e^{2}}{8 \pi \varepsilon_{0} a} .
$$


For speeds much lower than the speed of light, the member $\frac{1}{4} \frac{v^{4}}{c^{4}}$ may be neglected, so

$$
W_{v}=\frac{4}{3}\left(1+\frac{v^{2}}{c^{2}}\right) \frac{e^{2}}{8 \pi \varepsilon_{0} a} .
$$

The relationship between the fields of energy and rest mass of the electron is given by [4]

$$
W=\frac{4}{3} \frac{e^{2}}{8 \pi \varepsilon_{0} a}=m c^{2},
$$

Consequently, additional energy of electron, connected with the fact that of its field they depend on speed, to be determined by the relationship

$$
W_{v}-W=W\left(\operatorname{ch}^{2} \frac{v}{c}-1\right) \approx m v^{2} .
$$

The same relationship is fulfilled for the transverse kinetic energy:

$$
T_{\perp}=W_{v}-W \approx m v^{2} .
$$

Thus it is apparent that entire additional electric field energy wholly proceeds with shaping of transverse kinetic energy; therefore it is possible to identify them, considering as the different manifestations of one and the same energy.

Came out extremely interesting result - transverse kinetic energy is approximately equal to the doubled longitudinal kinetic energy:

$$
T_{\perp} \approx 2 T .
$$

Question does arise, must not this equality be precise. If this in reality then, then it is possible to obtain simpler equality, carrying longitudinal and transverse kinetic energies to the separate coordinate axes.

Let us introduce indices $\alpha=\overline{1,3}, \beta=\overline{1,3}$, Kronecker symbol $\delta_{\alpha \beta}$. Let the radius-vector of electron in the system of rectangular Cartesian space coordinates with the unit vectors relating to this inertial reference system $\mathbf{e}_{\alpha}=\left(\delta_{\alpha \beta}\right)$ take the form $\mathbf{x}=\left(x_{\alpha}\right)$. Let us select the orientation of the system of space coordinates so that the unit vector $\mathbf{e}_{3}$ would be collinear to velocity vector $\mathbf{v}=\left(v_{\alpha}\right)$, i.e. $v_{\alpha}=\delta_{\alpha 3} v$. Let us introduce into the examination those referred to the concrete axis $\alpha=\overline{1,2}$ of space coordinates (coordinate-by-coordinate) kinetic mass $\tilde{m}_{1}=\tilde{m}_{2}=\tilde{m}_{\uparrow}$ and transverse kinetic energy $T_{\perp 1}=T_{\perp 2}=T_{\uparrow}$ as follows:

$$
T_{\uparrow}=\tilde{m}_{\uparrow} c^{2}=\frac{T_{\perp}}{2} ; \tilde{m}_{\uparrow}=\frac{m_{\perp}}{2} .
$$

Thus, occurs the simple equality:

$$
T_{\uparrow}=T .
$$

If this equality is actually precise, then is thus obtained the new fundamental physical law, which can be formulated as follows: coordinate-by-coordinate kinetic energies for the different space coordinates are equal to each other. This situation 
can be called kinetic balance, and law itself - by the law of conservation of kinetic balance.

New law (if it is valid) is one of the dynamic laws of conservation, together with the known even in classical, nonrelativistic physics laws of conservation of energy, pulse and the moment of momentum. These three known laws are derived from the requirement of the invariance of Lagrangian relative to three transformation groups of the coordinates: the law of conservation of energy is derived from the invariance of Lagrangian relative to the group of temporary displacements (property of the uniformity of time), momentum conservation - of the three-dimensional translations (property of the uniformity of space), the moment of momentum - of threedimensional rotations (property of the isotropy of space). In this case in classical nonrelativistic physics with the examination of space and conversion time of Galileo they always stood by house, about which mentioned already Minkowski in his famous report "space and time". In view of this, from the requirement of the invariance of Lagrangian relative to the transformation group of Galileo or some of the subgroup of this group no dynamic law of conservation is derived. Into STR Galileo conversions they were substituted with the conversions of Lorenz, and already from the requirement of the invariance of Lagrangian relative to the Lorenz conversionsis obtained its dynamic law of conservation, connected with the appropriate components of the tensor of energy-momentum. Now time is alien to fill this missing gap, after introducing the law of conservation of kinetic balance.

In the Lagrange formalism of classical nonrelativistic the physicists of the conversion Galileo are considered very uniquely in comparison with other transformation groups of coordinates. Namely, it is possible to isolate two aspects of this calculation. It is first, considered that the Galileo conversions are one of the manifestations of the isotropy of space, in consequence of which is advanced one of the requirements for Lagrangian - it must depend only on the absolute value of speed, but not of direction. It is in the second place, considered that the Galileo conversions reflect the physical law of relativity of Galileo, in consequence of which is advanced another requirement for Lagrangian - it must reduce to the equations of motion, invariant relative to data of conversions. A drawback in this approach is the fact that the space and time in classical nonrelativistic physics are considered as the independent forms of existence of material, not connected into the united space-time. In the theory of relativity the space and time are considered connected with each other into the common space-time continuum with the united certificate (pseudo-Euclidean into STR and pseudo-Riemann with the specific curvature in General theory of relativity), thanks to which this certificate can be introduced directly into Lagrangian, ensuring its invariance relative to the appropriate transformations of coordinates of the type of the Lorenz conversions.

In time-spatial giperkontinuum already there is no united certificate. It is the hierarchically structured totality of the infinite set of particular space-time continua with its own certificate and level conversions of Lorenz. This does not make it possible to introduce some certificate of united space-time directly into Lagrangian. Nevertheless, in giperkontinuum are the transformations of coordinates, which play in it fundamental role, - the Galileo conversions, since they treat as the level 
Lorenz conversionsof infinitely high level. Thus, space and time are not the independent forms of existence of material, but they are connected into united giperkontinuum by the Galileo conversions, which, for this reason, must play more fundamental role in the Lagrange formalism.

In the group of all possible Galileo conversions it is possible to separately isolate the subgroup of those Galileo conversions, which leave constant the module of speed, changing only its direction (izovelotsitar Galileo conversions). In classical nonrelativistic physics Lagrangian must be invariant relative to precisely such Galileo conversions. However, with independent examination of space and time characteristic for such a physics the subgroup of the izovelotsitar Galileo conversions actually identifies with the group of three-dimensional rotations. This is the base of the fact that the Galileo conversions are considered one of the manifestations of the isotropy of space. But in connection with to giperkontinuum this identification hardly can be considered justified, the isotropy of giperkontinuum should be distinguished from the Isotropy of space, and reflecting Isotropy giperkontinuum the izovelotsitar Galileo conversions - from the reflecting the isotropy of space threedimensional rotations.

Thus, in giperkontinnum besides the requirements of the uniformity of time, the uniformity of space and isotropy of space to Lagrangian should be imposed even the requirement of the Isotropy of giperkontinuum. Thus, to the invariance of Lagrangian relative to temporary displacements, three-dimensional translations and three-dimensional rotations must be added invariance relative to the izovelotsitar Galileo conversions. As will be shown below, precisely, the invariance of Lagrangian relative to the izovelotsitar Galileo conversions it leads to the law of conservation of kinetic balance.

In classical nonrelativistic physics Lagrangian of particle in the inertial reference system takes the form:

$$
L=T-U,
$$

where $T=m v^{2} / 2$ - the kinetic energy, $U$ - potential energy of particle.

Lagrangian is determined with an accuracy to by complete time derivative of the arbitrary function of coordinates and time. The addition of this function to Lagrangian does not influence the form of equations of motion, although it leads to another expression for the energy of particle. In particular, from Lagrangian it is possible to deduct a certain constant, having sense of rest energy of particle. If we take energy fields on as this constant $W=m c^{2}$, that Lagrangian of signs the form:

$$
L=T-W-U \text {. }
$$

The total energy of the particle corresponds to this Lagrangian

$$
W_{T}=\frac{\partial L}{\partial v} v-L=\frac{m v^{2}}{2}+m c^{2}+U=T+W+U .
$$

One should separately emphasize that with this examination and the mass, and Lagrangian, and total energy, and kinetic energy are scalars. With the examination of similar Lagrangian in giperkontinuum it is necessary such scalar quantities to replace by the appropriate vector:

$$
\begin{aligned}
L_{\alpha} & =T_{\alpha}-W-U . \\
& -166-
\end{aligned}
$$


If the different components of the vector of Lagrangian are not equal to each other, then the turning of velocity vector will lead to the turning of the vector of Lagrangian. Therefore the invariance of the vector of Lagrangian relative to the izovelotsitar Galileo conversions indicates the equality between themselves of all three components of the vector of Lagrangian, and, therefore, also all three components of the vector of kinetic energy. Rectilinear motion with a constant velocity vector $\mathbf{v}=\left(\delta_{\alpha 3} v\right)$ occurs only with $U=0$ and $T_{3}=T$. In view of the internal symmetry of motion, which is expressed in its invariance relative to the transposition of the first two space coordinates, we have: $T_{1}=T_{2}=T_{\uparrow}$. The equality between themselves of the components of the vector of kinetic energy now indicates $T_{\uparrow}=T$. If longitudinal kinetic energy is formed by means of the speed, then transverse - by means of increase in the transverse mass. As a result, the components of entering the vector Lagrangian of the vector of kinetic energy will take the form:

$$
T_{1}=\tilde{m}_{1} c^{2} ; T_{2}=\tilde{m}_{2} c^{2} ; T_{3}=T=\frac{m v^{2}}{2} .
$$

The equality between themselves of these values is exactly the law of conservation of kinetic balance.

Thus, speaking about the value of kinetic energy, it is possible not to refine, with what precisely kinetic energy the discussion deals: by longitudinal or coordinateby-coordinate transverse; nevertheless they are equal to each other. Since transverse mutually orthogonal directions only two, then summary transverse kinetic energy is equal to the doubled coordinate-by-coordinate transverse kinetic energy, it is equal to the doubled longitudinal kinetic energy, and it is equal to the additional energy, connected with the dependence of field on the speed:

$$
W_{v}-W \approx 2 T \text {. }
$$

Thus, is established the physical cause for the presence in the moving material bodies of kinetic energy, and, therefore, also their inertia properties (both longitudinal and transverse). These properties are connected with the dependence of the scalar potential of the charges, of which consist all material bodies, from the relative charge rate. An increase in the transverse kinetic energy is caused by an increase in the transverse component of field. Energy of increase in the transverse component of field due to the nontrivial speed of motion exactly is equal to transverse kinetic energy.

The establishment of the physical cause for inertia and kinetic energy makes it possible to establish also the physical cause for the dependence of the scalar potential of electric charge on the speed. The it turned out that this dependence is the direct consequence of the new law of conservation of the kinetic balance, caused by the invariance of Lagrangian relative to the izovelotsitar Galileo conversions.

But the establishment of the physical cause for the dependence of the scalar potential of electric charge on the speed makes it possible to refine the concrete form of this dependence, and the, and also, therefore, corresponding dependences on the speed of the tension of electric field and transverse mass: 


$$
\phi_{\perp}=\phi \sqrt{1+\frac{v^{2}}{c^{2}}} ; E_{\perp}=E \sqrt{1+\frac{v^{2}}{c^{2}}} ; m_{\perp}=m\left(1+\frac{v^{2}}{c^{2}}\right) .
$$

The precise dependences of scalar potential and tension of electric charge on the speed do not contradict the trans-coordinate formulation of electrodynamics, since formulas (17.71) establish the form of the trans-coordinate derived field functions only of first order, leaving opened a question about the derivatives of higher order. In this case of formula (17.71) they remain the same.

Introduction into physics of the law of conservation of kinetic balance conducts to radical reconstruction not only of electrodynamics, but also generally physics as a whole, but in particular, mechanics. Since present monograph is dedicated to electrodynamics, questions of such global of the reconsideration of physical ideas about the peace exceed the limits of its thematic. Nevertheless, to give one exponential example is nevertheless appropriate.

As this example let us examine the dynamics of uniform rotatory mechanical motion around our axis of the uniform cylinder of a radius $R$ with the module of the angular velocity $\omega$. If in the state of rest this cylinder has a mass $m$, that in rotation state its mass in the direction of axis will increase to the certain value $m_{\perp}$ This means that force $\mathbf{F}$, acting in the direction of the axis of the revolving cylinder (it can have different physical nature, including gravitational), it is capable of giving to it in this direction acceleration $\mathbf{F} / m_{\perp}$, which it will be on the module less $m_{\perp} / m$ once of that acceleration $\mathbf{F} / m$, which it reports the same force to the cylinder in the absence of its rotation.

This constant of proportionality is determined by the following formula:

$$
m_{\perp} / m=\frac{2}{R^{2}} \int_{0}^{R} r\left(1+\frac{\omega^{2}}{c^{2}} r^{2}\right) d r=1+\frac{\omega^{2} R^{2}}{2 c^{2}} .
$$

Physical the effect examined ensures an increase in the sluggishness of the revolving bodies, however, up to now this physical phenomenon it did not have satisfactory theoretical explanation. Physical nature of this phenomenon now not only explained, but also were obtained the corresponding calculation formulas. Although the effect is very small, for the corresponding improvement of the experimental technique it can be used for the experimental confirmation of the law of conservation of kinetic balance. From this law follows the electrodynamics, at the basis of dynamic laws of which lies the dependence of the scalar potential of electric charge on the speed. And from the same law follows the mechanics of high (relativistic) speeds, at basis of which lies another (giperkontinual) formulation of second Newton's law.

Let the force $\mathbf{F}$ transfer to material point by the mass $m$, of that moving with the acceleration rate $\mathbf{v}$, a. Then second Newton's law in classical nonrelativistic physics does not contain speed and is written in the form

$$
\frac{\mathbf{F}}{m}=\mathbf{a} \text {. }
$$


However, in time-spatial giperkontinuum this formula is carried out only approximately in the case of small (nonrelativistic) speeds, i.e., with $v<<c$, since in giperkontinuum are distinguished longitudinal mass $m$ and transverse mass

$$
m_{\perp}=m\left(1+\frac{v^{2}}{c^{2}}\right) .
$$

Longitudinal and transverse directions here and throughout they are determined with respect to the direction of velocity vector $\mathbf{v}$.

For the convenient idea of second Newton's law in time-spatial giperkontinuum it is expedient to use calculation of quaternions with the collection of the imaginary units $\mathbf{i}, \mathbf{j}, \mathbf{k}$. Let us assume the unit vectors $\mathbf{e}_{1}, \mathbf{e}_{2}, \mathbf{e}_{3}$ of the system of the rectangular Cartesian space coordinates of this inertial reference system to be equal:

$$
\mathbf{e}_{1}=\mathbf{i}, \mathbf{e}_{2}=\mathbf{j}, \mathbf{e}_{3}=\mathbf{k}
$$

In this case the radius-vector $\mathbf{x}$ occurs purely vector quaternion, i.e., by quaternion with the zero real part)

$$
\mathbf{x}=x_{1} \mathbf{i}+x_{2} \mathbf{j}+x_{3} \mathbf{k} \text {. }
$$

Force $\mathbf{F}$ and acceleration $\mathbf{a}$ are spread to appropriate mutually orthogonal longitudinal $\mathbf{F}_{\uparrow}, \mathbf{a}_{\uparrow}$, and $\mathbf{F}_{\perp}, \mathbf{a}_{\perp}$ - transverse components:

$$
\mathbf{F}=\mathbf{F}_{\uparrow}+\mathbf{F}_{\perp} ; \mathbf{a}=\mathbf{a}_{\uparrow}+\mathbf{a}_{\perp},
$$

moreover $\mathbf{F}, \mathbf{a}, \mathbf{F}_{\uparrow}, \mathbf{a}_{\uparrow}, \mathbf{F}_{\perp}, \mathbf{a}_{\perp}, \mathbf{v}$ similar to radius-vector $\mathbf{x}$ they are also purely vector quaternions.

The quaternions of longitudinal and transverse accelerations are expressed as the quaternions of acceleration and speed as follows:

$$
\mathbf{a}_{\uparrow}=\left(\operatorname{Re} \frac{\mathbf{a}}{\mathbf{v}}\right) \mathbf{v} ; \mathbf{a}_{\perp}=\mathbf{a}-\left(\operatorname{Re} \frac{\mathbf{a}}{\mathbf{v}}\right) \mathbf{v} .
$$

Taking into account these two quaternions second Newton law in time-spatial giperkontinuum in the language of the calculation of the quaternions of signs the form:

$$
\mathbf{F}=m\left(\operatorname{Re} \frac{\mathbf{a}}{\mathbf{v}}\right) \mathbf{v}+m_{\perp} \mathbf{a}-m_{\perp}\left(\operatorname{Re} \frac{\mathbf{a}}{\mathbf{v}}\right) \mathbf{v} .
$$

Expressing the transverse mass through the longitudinal, finally we will obtain:

$$
\frac{\mathbf{F}}{m}=\left(1+\frac{v^{2}}{c^{2}}\right) \mathbf{a}-\frac{v^{2}}{c^{2}}\left(\operatorname{Re} \frac{\mathbf{a}}{\mathbf{v}}\right) \mathbf{v} .
$$

With $v<<c$ this formula converts to traditional formula for this law. However, in the general case it is possible to establish even no coincidence of the directions of the vectors of force and acceleration. 


\section{$\S 23$. Special features of the mathematical apparatus for the classical electrodynamics}

Vector analysis is the basic mathematical apparatus for electrodynamics. Such vector quantities, as force, speed, acceleration, electric field and current demonstrate well the physical nature of these values. However, with the use of a vector apparatus for describing the physical processes are introduced such of vector, which do not reflect the physical essence of those processes, which they describe. We will call such vectors vector- phantoms. Let us give several examples.

If is located the disk, which revolves with the angular velocity $\omega$, then they depict this process as the vector, which coincides with the rotational axis of disk and rests in its center. It does ask itself, is there this vector in reality and that it does represent? There is no doubt about the fact that this vector can be introduced by arrangement, but any physical sense as, for example, velocity vector, it does not have. Thus the vector of momentum is accurately introduced. This vector also coincides with the rotational axis, it rests in the center of the plane of rotation and it is equal to the work of radial velocity to a radius. Similarly is introduced the vector of the magnetic dipole moment, which for the ring current is equal to the work of the current strength to the area of the circle streamlined with current. This vector coincides with the rotational axis of circle and rests on its plane. But any physical sense these a vector do not have.

Let us recall what is the vector is, which presents rotor. This vector is introduced as follows

$$
\operatorname{rot} \mathbf{a}=\left(\begin{array}{ccc}
\mathbf{i} & \mathbf{j} & \mathbf{k} \\
\frac{\partial}{\partial x} & \frac{\partial}{\partial y} & \frac{\partial}{\partial z} \\
a_{x} & a_{y} & a_{z}
\end{array}\right)
$$

in order to explain the geometric sense of rotor let us examine solid body, which revolves with the angular velocity $\omega$ around the axis $z$. Then the linear speed of the body $v$ at point $(x, y, z)$ is numerically equal

$$
v=\omega r=\omega \sqrt{x^{2}+y^{2}},
$$

and component it along the axes, for the right-handed coordinate system, will be equal

$$
\begin{gathered}
v_{x}=-\frac{v y}{\sqrt{x^{2}+y^{2}}}=-\omega y, \\
v_{y}=-\frac{v x}{\sqrt{x^{2}+y^{2}}}=-\omega x, \\
v_{z}=0 .
\end{gathered}
$$

The vector components rot $\mathbf{v}$ in this case to be determined by the relationships

$$
\begin{gathered}
\operatorname{rot}_{x} v=\operatorname{rot}_{y} v=0, \\
-170-
\end{gathered}
$$




$$
\operatorname{rot}_{z} v=\frac{\partial v_{y}}{\partial x}-\frac{\partial v_{x}}{\partial y}=2 \omega .
$$

Is again obtained the vector, directed in parallel to rotational axis and normal toward the plane of rotation. This vector also is introduced by arrangement and of any physical sense it does not have. The same argument can be extended to the vector product.

Thus, with the use of vector analysis for describing the physical phenomena are introduced two types of vectors. The first of them represents the real physical of vector, which characterize physical quantity itself taking into account of its value and direction (for example, the vector of force, speed, acceleration, tension of electric field and current). Another category of vectors - this those of vector, which can be presented with the aid of the operation of rotor or vector product. These vector do not represent physical quantities and they are introduced by arrangement, being vector- phantoms. Specifically, the vector of such type includes magnetic field.

Magnetic field is introduced or with the aid of the rotor of the electric field

$$
\frac{\partial \mathbf{H}}{\partial t}=-\frac{1}{\mu} \operatorname{rot} \mathbf{E},
$$

or as the rotor of the vector potential

$$
\mathbf{H}=\operatorname{rot} \mathbf{A}_{H} .
$$

This means that the magnetic field is not physical field, but represents the certain vector symbol, which is introduced by arrangement and of physical sense it does not have.

However, that does occur further? During writing of Maxwell equations rotor from the magnetic field they make level to the full current

$$
\operatorname{rot} \mathbf{H}=\operatorname{rot} \operatorname{rot} \mathbf{A}_{H}=\mathbf{j}_{\Sigma}
$$

is obtained so that rotor from the vector $\mathbf{H}$, which is introduced by arrangement, gives the real physical vector of current density. Thus, the vector of magnetic field represents typical vector- phantom.

It is possible to give another example. The Lorentz force, which acts on the moving charge, is determined by the vector product of the real velocity vector and of magnetic field:

$$
\mathbf{F}=\mu[\mathbf{v} \times \mathbf{H}]
$$

is again obtained so that the operation of vector product, which itself physical sense does not have, with the participation of real vector and vector of phantom it gives real physical force taking into account of its value and direction. Of this consists the sense of introduction in vector analysis of such operations as rotor and vector product. If we look to the mathematical apparatus for physics in connection with to vector analysis, then it appears that this apparatus represents the mixture of real physical vectors and vectors of the phantoms, the relation between which it is regulated with the aid of the, including and operations indicated. 
But then appears a question, and is it possible generally to exclude from the custom the vector- phantoms, which introduce only by arrangement and to build electrodynamics without their use. Above it was convincingly shown that entire electrodynamics can be built without the use of this concept as magnetic field. In this case as its basis must be assumed such fundamental concepts as the electric field, which is the gradient of scalar potential, and also the vector potential, by the reason for appearance of which there is dependence of the scalar potential of charge on the speed of its relative motion. This approach - this is revolution in the classical electrodynamics. It places in the chapter of classical electrodynamics the scalar potential of charge and its dependence on the speed and deprives the magnetic field of the rights of physical field.

The concept of magnetic field as real vector exists since scientific they noted, how organizationally the iron shavings near the magnets or the annular currents behave. This behavior seemed by the almost obvious consequence of the presence of some force vector, which acts on them. And this vector acknowledged magnetic field. However, obvious is not always real. It occurs that this behavior of the iron shavings is connected not with existence of magnetic field as physical material field, but with the fact that currents possess potential energy according to the relation to friend to the friend, and this energy for the case of stable equilibrium is always approached the minimum. Specifically, for this reason the iron shavings, in which separate atoms present microscopic annular currents, and behave thus.

\section{$\S 24$. Mechanical electrization}

Earthquakes are accompanied by the appearance of electrical phenomena which they did not up to now find its explanation. In 1847 before the earthquake into Sinsyu (Japan) against the background of dark sky arose the revolving fiery cloud. It moved to the side of mountain it was ide and, its reach, it disappeared. In 1911 on the eve of the earthquake in Germany in the cloudless sky appeared the fireball, and 26 November 1930 before the earthquake in before the earthquake in peninsula Idzu (Japan) of the aurora borealis. Survived Ashkhabad tragedy 1948 they say, that on the eve of the earthquake they saw the arc from the electrical discharges flying on the sky, then, immediately after wind gust, it was heard the first underground push. During the Tashkent earthquake 1966 from under the earth was pulled out the gigantic luminous torch, it swiftly rose upward and it was dissolved in air. In 1976 occurred super-power Tien-Shan earthquake, during which light flashes they were controlled hundreds of kilometers from the epicenter of earthquake.

Earthquake begins from break and displacement of the rocks in the depth of the Earth [62-65]. This place is called seismic center or hypocenter. Its depth usually is not more than $100 \mathrm{~km}$, but sometimes it reaches also $700 \mathrm{~km}$. In some cases the layers of the earth, situated along the sides of breaking, are moved to each other. In others - the earth on one side of breaking descends, forming discharges. Underwater earthquakes are the reason for the tsunami, of the long waves, generated by powerful action on entire thickness of water in the ocean, during which occurs the 
sharp shift (raising or lowering) of the section of the sea bottom. Tsunami are formed with the earthquake of any force, but large forces those, which appear as a result of the strong earthquakes, reach. The sharp displacement of the large masses of the earth in the seismic center is accompanied by the mechanical impact of colossal force. The energy, isolated with such impacts, can repeatedly exceed energy of the nuclear explosions [66]. It is natural that such processes are accompanied by colossal mechanical voltage and powerful gaps of the layers of species. From the seismic center the seismic waves, which are also characterized by periodic mechanical compression and tension of layers and rocks, are propagated. Sometimes earthquakes are accompanied by the appearance of lightning. From the aforesaid follows the consequence about the fact that the electrical phenomena, which accompany earthquakes, can be connected with the mechanical processes in the layers of species.

It follows from the carried out examination that also the appearance of rapid (impact) mechanical loads also must lead to the appearance in the isolated metallic model of pulse potential. This question was investigated on the installation, whose schematic was given in Fig. 43.

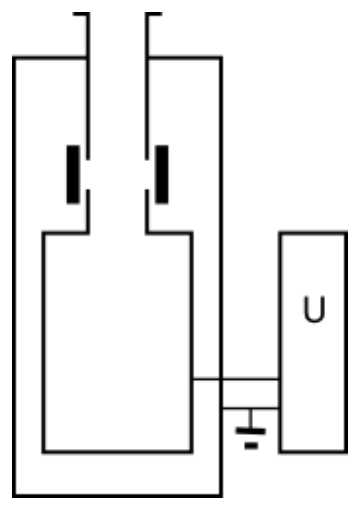

Fig. 43. Installation diagram for investigating the appearance of the pulses of electric field with the impact

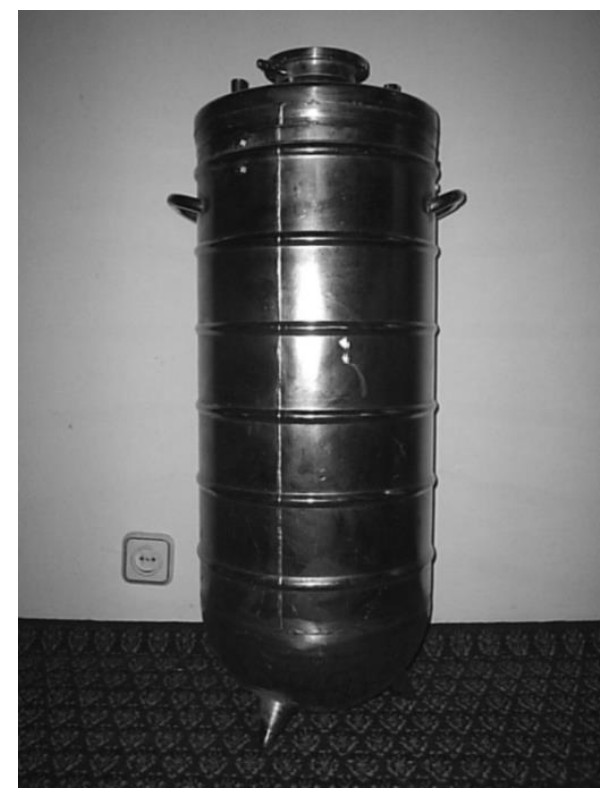

Fig. 44. The common form of installation for investigating the dynamic loads 


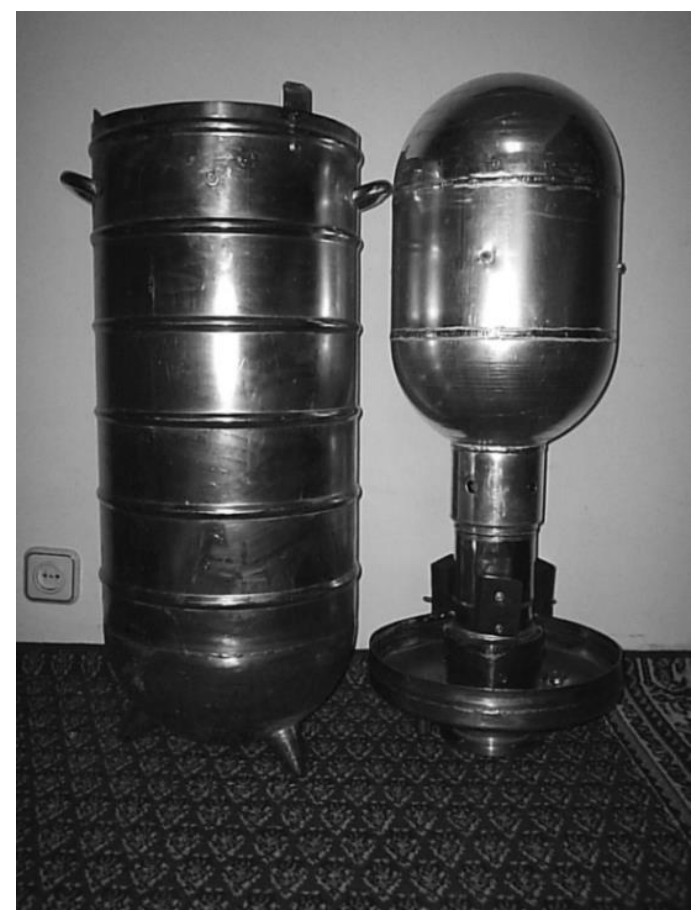

Fig. 45. Type of installation in the dismantled form

Internal capacity is suspended to the external screen with the aid of wide neck. For eliminating the galvanic contact between the external screen and the internal capacity the neck has a section. Odd parts of the neck are connected by the insulating plates, which in the figure are designated by the short black sections of lines. Internal capacity is prepared from aluminum in the form of flask, its end walls are executed in the form hemispheres. This construction of end walls is necessary in order to avoid their severe strain with the realization of the explosions of explosive in the internal capacity. Common form installations for investigating the dynamic loads on the aluminum flask and the component parts of the installation are shown in Fig. 44 and Fig. 45.

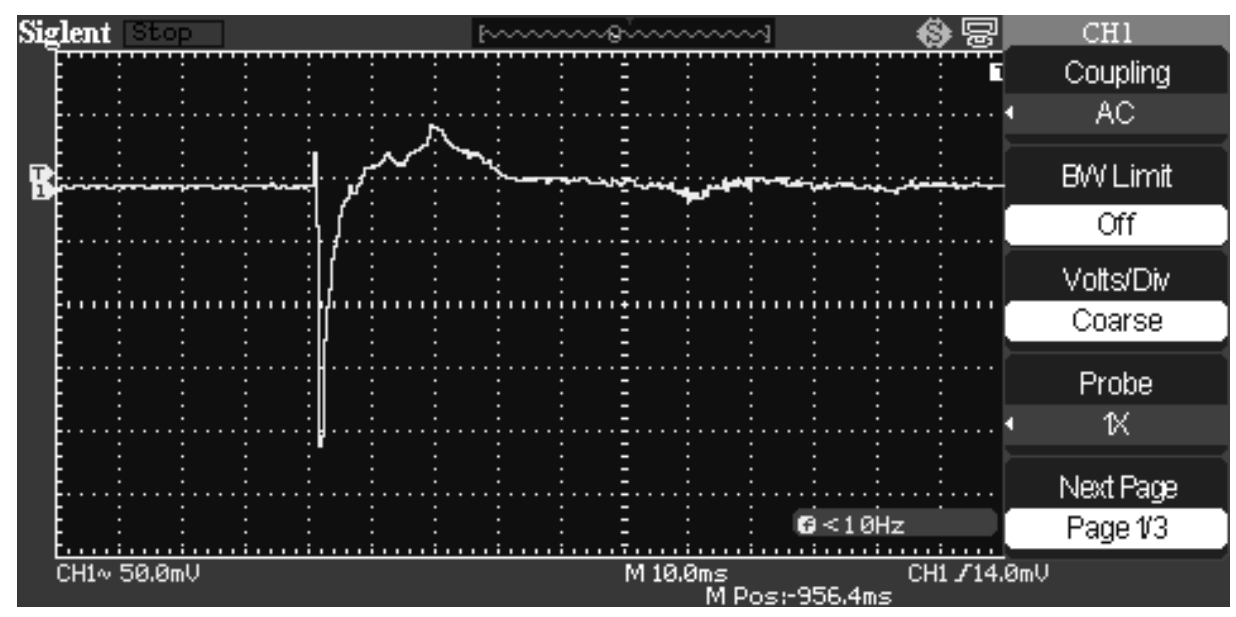

Fig. 46. Shape of pulse after a drop in the rod on the bottom of internal capacity 
During the inclusion into neck from a height $1 \mathrm{~m}$ of the bottom of the internal capacity of the rod with a weight $200 \mathrm{~g}$ between the external screen and the internal capacity is observed the voltage pulse, shown in Fig. 46.

In order to avoid to the appearance of additional pulses with a lateral drop in the rod after the impact of its end about the bottom of flask, the side of rod is wound by soft tissue. Data of this experiment correspond to the experimental data, obtained with the copper flask, the code its tension led to the appearance on the flask of negative potential. With the impact of the end of the rod about the bottom of flask also occurs the local deformation of its bottom, with which in the point of impact occurs the tension.

If we inside the aluminum flask explode the charge of small value, then is observed the voltage pulse, shown in Fig. 47.

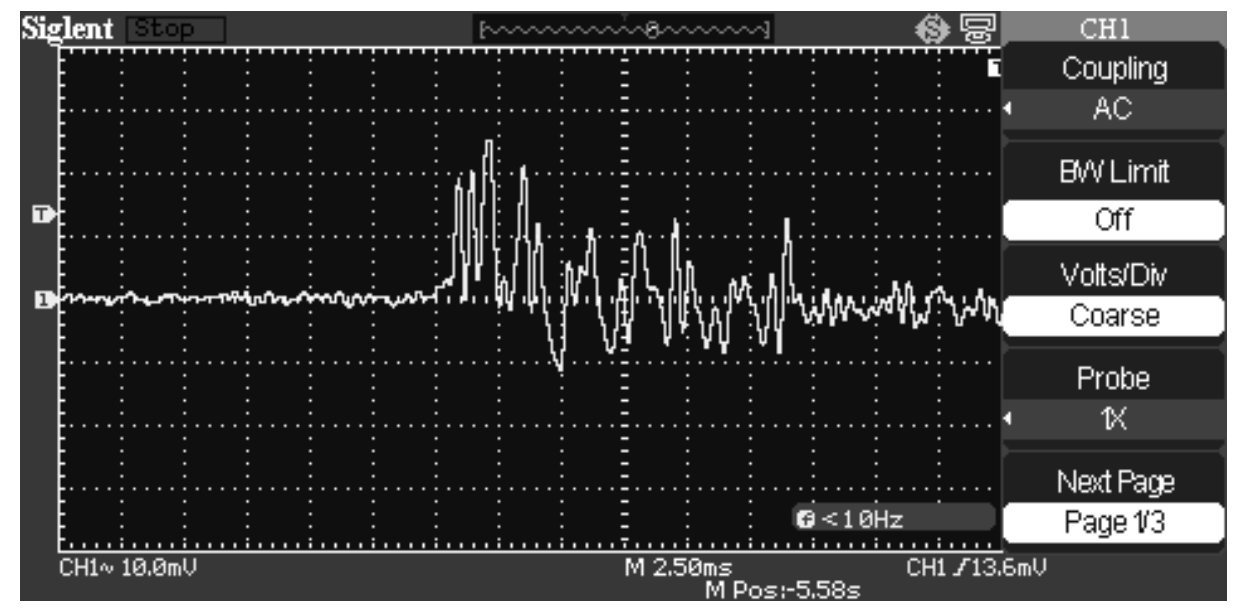

Fig. 47. The form of the voltage pulses, obtained with the explosion of explosive in the aluminum flask

The heteropolar repetitive pulses, which are been the consequence of the multiple reflection of shock wave from the walls of the flasks, which lead to its deformation, are observed in the oscillogram, in this case there are pulses corresponding to both the tension of the walls of flask and to their compression.

If we into the aluminum flask place the spring, isolated from the flask, and to force it periodically to be compressed, then potential on the flask also bears periodic nature. The experiment indicated was conducted according to the diagram, depicted in Fig. 48.

To the cotton cord, which emerges outside flask, is fastened the spring, from which is suspended the load. This system is had the mechanical resonance, whose resonance frequency, determined by spring constant and by cargo weight. If we toward the end thread exert periodic force at the frequency of resonance, then it is possible to attain the periodic deformation of spring at this frequency with in effect constant position of load. 


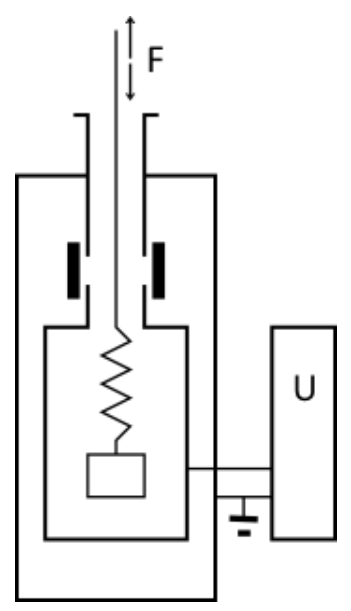

Fig. 48. Diagram of experiment with the spring

The dependence of electric potential on the flask, obtained in this experiment, it is shown in Fig. 49.

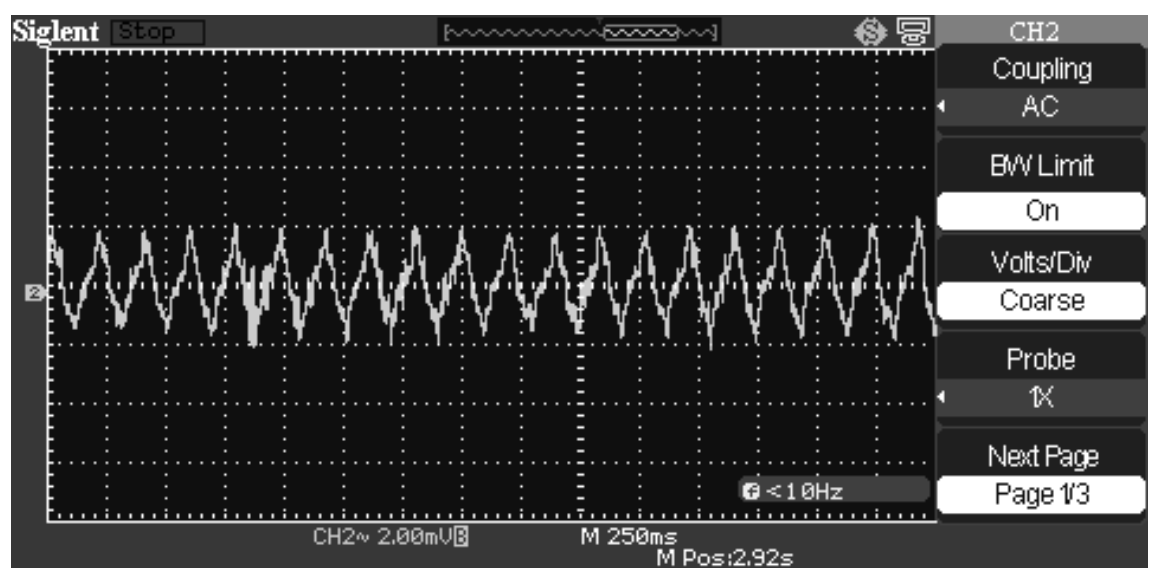

Fig. 49. An alternation in the potential on the flask

Obtained data attest to the fact that in the process of the deformation of spring, in the flask the alternating unitary charge is formed.

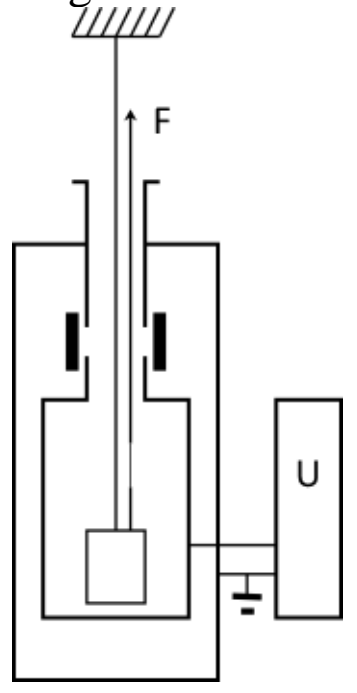

Fig. 50. Experiment on the break inside the flask of copper wire 
If we inside the flask tear thin copper wire, then the voltage pulse also is observed between the flask and the external screen. This experiment was conducted according to the diagram, shown in Fig. 50.

The load is suspended inside the flask from the cotton cord. In parallel with the thread, from which is suspended the load, is located another kapron thread, in break of which is fixed the section of the copper wire with a diameter $0.3 \mathrm{of} \mathrm{mm}$. At the moment of the break of the wire between the flask and the external

Screen is observed the pulse, depicted in Fig. 51.

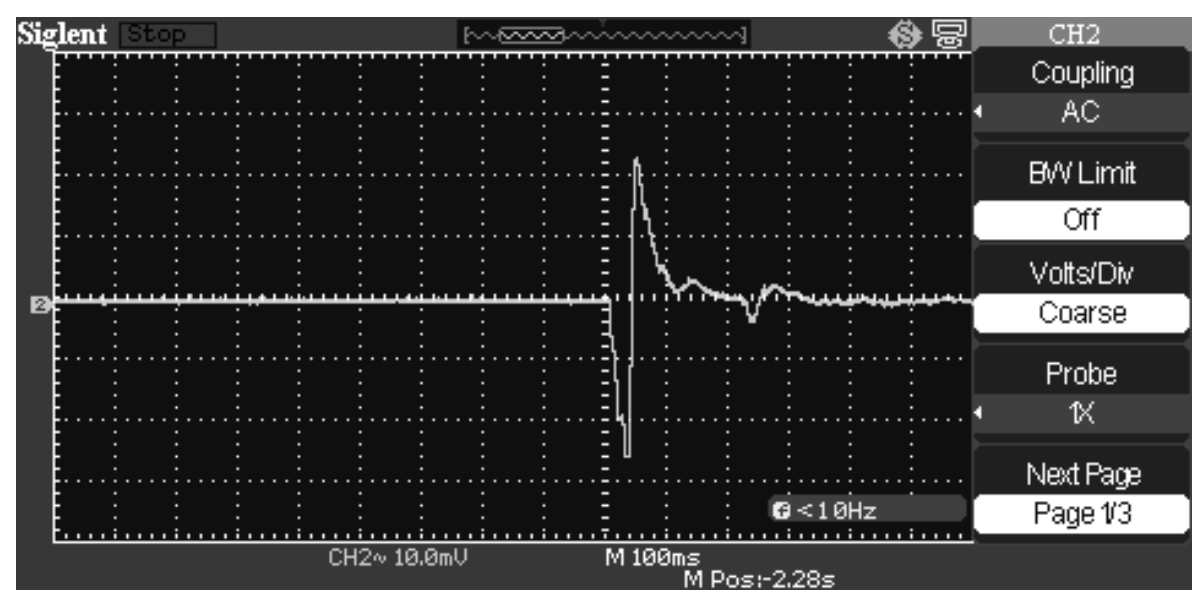

Fig. 51. Pulse, obtained with the break of wire

The negative part of the pulse corresponds to the tension of wire, which precedes its break. The positive part of the pulse corresponds to relaxation of deformation voltage two parts of the torn wire.

In such a manner both the mechanical deformation of wire and its break it is accompanied by the appearance of unitary charge inside the flask.

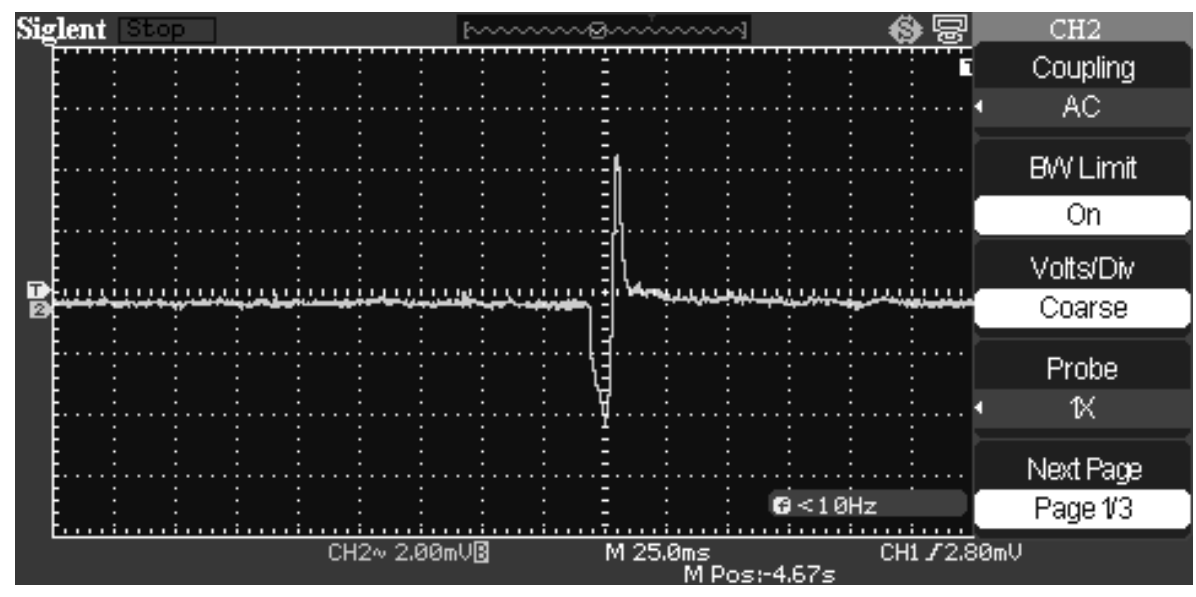

Fig. 52. With the break of silk thread is observed the oscillogram

Electrization appears also with the mechanical dielectric strains. If we conduct experiment with the dielectrics employing the procedure, depicted in Fig. 50, it is possible to obtain the following results. With the break of silk thread is observed the oscillogram, given in Fig. 52. 
In Fig. 53 is depicted the oscillogram, observed with the break of thread from vinyl chloride.

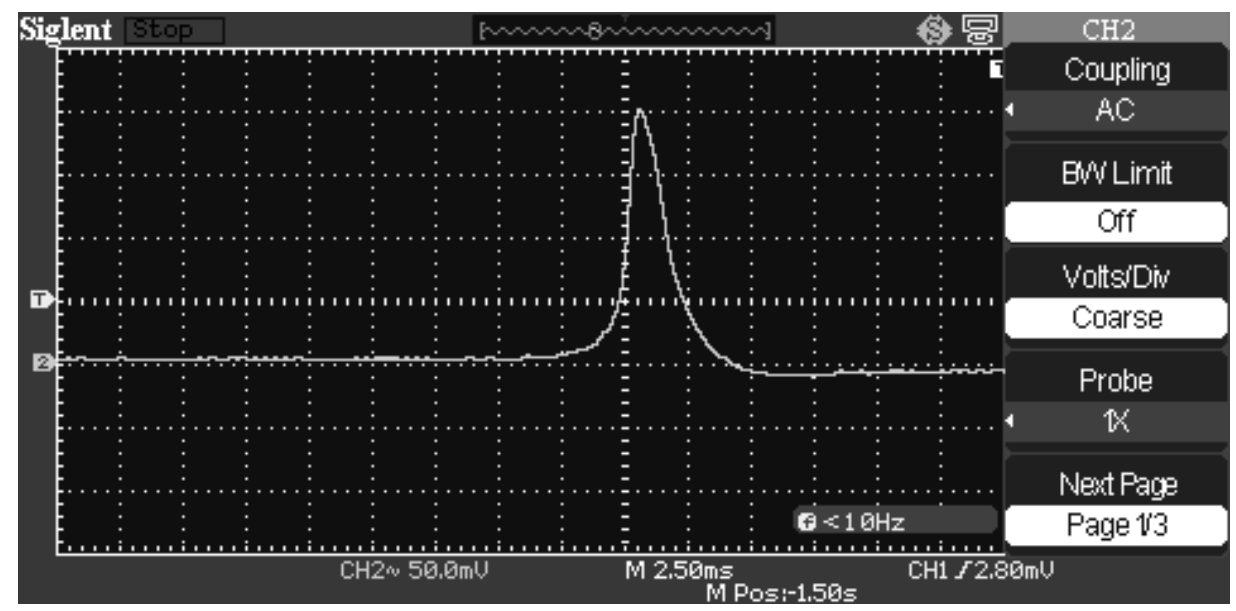

Fig. 53. Is depicted the oscillogram

If we as the thread use the lace, interlaced from the synthetic fibers, and to subject to its periodic mechanical loads, then will be obtained the oscillogram, given in Fig. 54.

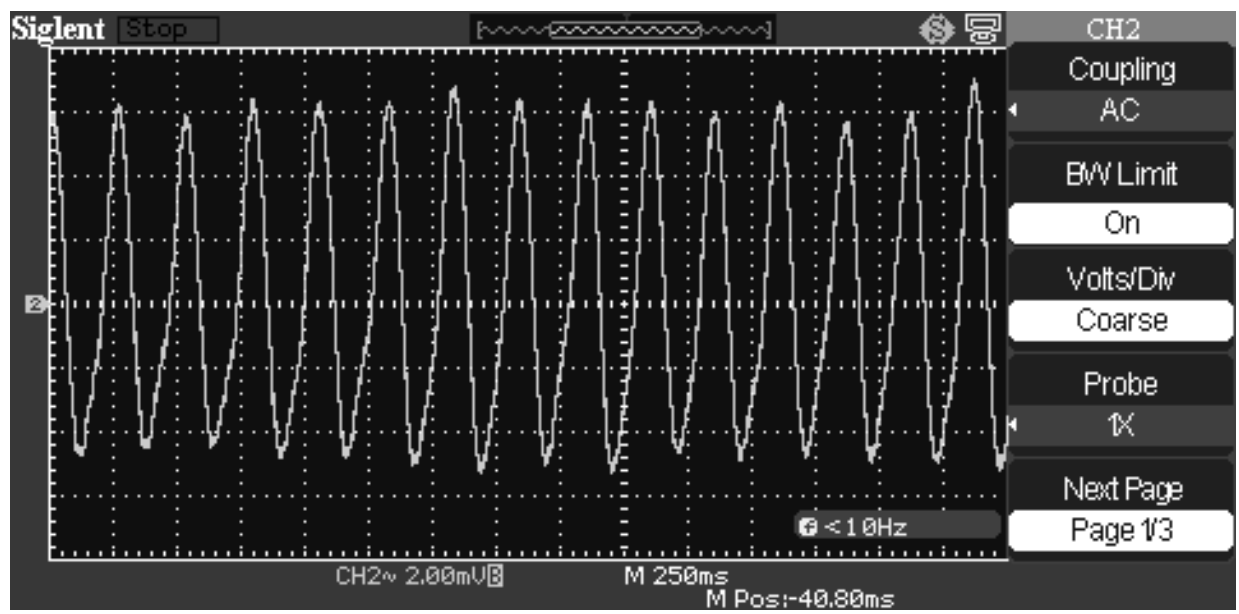

Fig. 54. Oscillogram, observed during application to the lace of periodic mechanical loads

Such properties of dielectrics earlier in the scientific publications are not described. Obtained experimental data speak, that by the way of compression, tension or destruction of conductors and dielectrics, placed in Faraday cage, it is possible to obtain in it the unitary charge of different signs, whose fields without difficulty penetrate through the metal screen of Faraday cage. Friction between the separate threads of dielectric generates the same effect, about than testify the experiments with the lace, made from such threads.

The electrization of models with their mechanical deformations was observed also in the works $[67,68]$. 


\section{PART III \\ OTHER NEW HYPOTHESES, EXPERIMENTAL RESULTS AND THE TECHNICAL SOLUTIONS}

\section{CHAPTER 5 \\ OTHER HYPOTHESES, EXPERIMENTAL RESULTS AND THE TECHNICAL SOLUTIONS}

\section{$\S 25$. Is laser quantum generator?}

The lasers are considered as the quantum generators. It is known that the laser emission possesses high coherence and directivity. Known in radio engineering principle of the construction of radiating systems with such characteristics - the use of a large quantity of elementary phased emitters, located in the determined order. Such systems are called the phased-array antenna (FA). Moreover, the greater the quantity of elementary sources it is used and the greater the dimensions of space, on which they are located, the greater the directivity and the radiated power can be obtained. For obtaining the high directivity the linear dimensions of system must be considerably more than the length of radiated wave.

In the work substance of laser also always is contained a huge quantity of elementary sources, which the atoms or the molecules of work substance are. If the discussion deals with the solid-state lasers, for example on the basis of ruby, then the radiating atoms, which are the atoms of chromium, it is also located in the crystal of work substance in the strictly defined order. If such atoms are synchronously excited by any means then so that their fluctuations would be phased as in phased-array antenna, then from a radio-technical point of view this system can give the very narrowly-directed emission, since. a quantity of emitters is very great, and the length of radiated wave is much less than the linear dimensions of the field of emission. But as to excite atoms? One of such methods - the collision excitation, when the work substance of laser they irradiate by short pulse from the flashbulb, as is done in ruby laser. But with the aid of the flashbulb it is not possible to excite atoms so that the phase of their fluctuations would correspond to the conditions of phased-array antenna. For this selection external macroscopic resonator serves. Selection indicated condition - agreement of one of the resonance modes of external resonator with the natural vibration frequency of the atoms of chromium. But this it is insufficient. The phasedarray antenna will compose only the atoms of chromium, located in the places, where their phase of fluctuations coincides with the phase of the fluctuations of macroscopic resonator. But in a total quantity of excited atoms of such it is not much, a total of several percentages of a total quantity. Therefore efficiency in ruby laser is low the efficiency.

Consequently, this generator works according to all laws of electrodynamics and radio engineering, and there is nothing in it quantum, although the name in it very beautiful - two-level quantum generator. 
The multilevel quantum generators, which radiate by lowering downward consecutively through several levels of quanta from the higher levels, work according to the radio-technical laws of nonlinear parametric systems. Occurs either parametric strengthening or parametric generation according to relationships To menli-Rou - to the energy relationships, which characterize interaction of fluctuations or waves in the nonlinear systems with the concentrated or distributed parameters:

$$
\sum_{m=1}^{\infty} \sum_{n=-\infty}^{\infty} \frac{m P_{m n}}{m \omega_{1}+n \omega_{2}}=0 ; \quad \sum_{m=-\infty}^{\infty} \sum_{n=1}^{\infty} \frac{n P_{m n}}{m \omega_{1}+n \omega_{2}}=0,
$$

where $P_{m n}$ - a change in the power at the combination frequency $m \omega_{H}+n \omega_{C}$, $\omega_{1}, \omega_{2}$ - the frequency of initial vibrations (waves). Relation $\omega_{2} / \omega_{1}$ must be irrational, since otherwise, it is possible to express all frequencies as the harmonics of one fundamental frequency.

The relationships of Manley -Rou are valid for the system with the arbitrary reactive nonlinear coupling. In conjunction with the laws of conservation of energy and momentum, they determine the nature of nonlinear interaction of waves (fluctuations) and make it possible to calculate the maximum effectiveness of frequency converter on the reactive nonlinearity.

Let per unit time it appear or disappear $A_{m n}$ the quanta of combination frequency. Then power at this frequency is equal

$$
P_{m n}=A_{m n} \cdot \hbar \cdot\left(m \cdot \omega_{1}+n \cdot \omega_{2}\right),
$$

In view of conservation of energy in the system total power is equal to zero:

$$
\sum_{m, n} P_{m n}=\sum_{m, n} \hbar A_{m n} \cdot\left(m \cdot \omega_{1}+n \cdot \omega_{2}\right)=0 .
$$

Since $\omega_{2} / \omega_{1}$ it is irrational, and $m, n, A_{m n}$ - integers, this equality is carried out, only if both terms are equal to zero:

$$
\sum_{m, n} m \cdot A_{m n}=\sum_{m, n} n \cdot A_{m n}=0 .
$$

After expressing $A_{m n}$ from (25.1) and after substituting into the last expression, we will obtain:

$$
\sum_{m, n} \frac{m \cdot P_{m n}}{m \cdot \omega_{1}+n \cdot \omega_{2}}=\sum_{m, n} \frac{n \cdot P_{m n}}{m \cdot \omega_{1}+n \cdot \omega_{2}} .
$$

Let us examine the relationships of Manley-Rou in the particular case trekhchastotnogo interaction. Let, for example, combination be the difference frequency $\omega_{0}=\omega_{1}-\omega_{2}$. Then system has three frequencies:

$$
\omega_{0}(m=1, n=-1) ; \quad \omega_{1}(m=1, n=0) ; \quad \omega_{2}(m=0, n=1) .
$$

In this case the relationships of Manley -Rou take the form:

$$
P_{0,1} / \omega_{2}=P_{1,-1} / \omega_{0}=P_{1,0} / \omega_{1} .
$$

They describe nonlinear medium with three interconnected resonances. Moreover if we excite one of the resonances, then I will be excited and rest. The energy, stored up in each of the resonances, is proportional to resonance frequency, that quantum mechanics interprets as the presence on Wednesday of the energy 
levels, proportional to frequency. But the processes of energy transfer of one resonance in another, which ensures the nonlinearity of medium, quantum mechanics interprets as triple from one energy level to another. This scholastic diagram hides the true physical sense of process.

The first, so-called, quantum generator, in which the electromagnetic vibrations OF SHF were generated with the aid of the molecules $\mathrm{NH}_{3}$, was created in 1954 Basov and Prokhorov. The generators worked on the molecular beam of ammonia. Its work thus was explained from the point of view of quantum mechanics: molecules $\mathrm{NH}_{3}$, possessing electrical dipole moment, flying through the heterogeneous electric field, are differently slanted by this field depending on their internal energy. In the first molecular generator the sorting system was the quadrupole capacitor, which consists of the parallel 4 rods, connected in pairs through one with the high-voltage rectifier. The electric field of this of capacitor is nonhomogeneous, it causes the bend of the trajectories of molecules, which fly along its longitudinal axis. Molecules, which are found in the upper energy state, are slanted to the condensor spindle and fall inside the cavity resonator, that are located in the lower - are rejected to the sides.

Falling inside the resonator, the excited molecules emit photons under the action of the field of resonator. Photon energy strengthens field in the resonator, increasing the probability of the stimulated emission for the molecules, which fly later (the feedback). If the probability of the stimulated emission of photon is more than the probability of absorption in the walls of resonator and emission in its limits, then the intensity of the field of resonator at the transition frequency rapidly grows due to the internal energy of molecules. Growth ceases, when field in the resonator reaches the value, with which the probability of the stimulated emission becomes so large, that in the transit time of resonator manages to emit photon exactly half of the molecules of beam. In this case for the beam as a whole the probability of absorption the equal probability of the stimulated emission becomes (saturation). The power of molecular generator with molecular beam $\mathrm{NH}_{3}$ is equal $10^{-11} \mathrm{~W}$.

But how in reality does work molecular generator with the molecular beam of ammonia? The molecule of ammonia has a dipole moment, and in the space this molecule can have two positions: one steady, but another - no. If with the thermal molecular excitation falls into the unsteady state, i.e. the electric dipole overturns and it begins to overturn with the resonance frequency. Since the size of dipole, determined by molecular dimension, is considerably lower than the wavelength of emission, this resonance has very high quality. If we with the aid of the quadrupole capacitor filter out molecules in the unsteady state, to and then throw in them into the macroscopic resonator, whose resonance frequency coincides with the frequency of the vibrations of molecular dipole, then such mole cules will excite in the resonator of fluctuation, and macroscopic resonator will independently select molecules with the phase of fluctuations, which coincides with the phase of fluctuations in the ger. Only difference from the ruby laser - in the latter the vibration frequency lies at the region of light frequencies, and in ammonium - in HF range. Therefore the principle of work of molecular genera- 
tor as ruby laser, is plotted in the radio-technical concepts without the attraction of quantum-mechanical concepts.

One should separately emphasize that in the existing constructions of lasers the selection of the atoms, which form the phased lattice, which forms the directed laser beam, achieves the macroscopic resonator, in which is located the active material. Macroscopic resonator is the separator, which selects only corresponding to the specified conditions oscillations of the atoms located in it. The excited by impact method atom, which presents dipole source, can be located in the resonator in any place, and its emission can have any polarization and direction. But only atoms with the frequency, which coincides with frequency of one of the modes of macroscopic resonator, have chances to give the contribution to the laser beam. For this it is necessary that the direction of the emission of these atoms would coincide with the axis of resonator, and so that the distance from the atoms to both lenses would be multiple to half of the wavelength of the emission of atom. Then the emissions of atom after multiple reflections from the mirrors of external resonator form exsponetsially the diminishing standing wave. If such atoms much, and they are excited in the different time, they all together form the nondamping standing wave between the mirrors, which, after traversing the semi-transparent mirror, gives laser beam of light. This beam, in view of Huygens's principle, weakly disperses if the size of semi-transparent mirror much more than wavelength, that also is observed in practice.

Atoms with the oscillations of those atoms, which do not satisfy these conditions, will consume their vibrational energy for the creation of the scattered noncoherent radiation. Because we see the bright glow of the transparent tube of gas laser, through which this emission leaves.

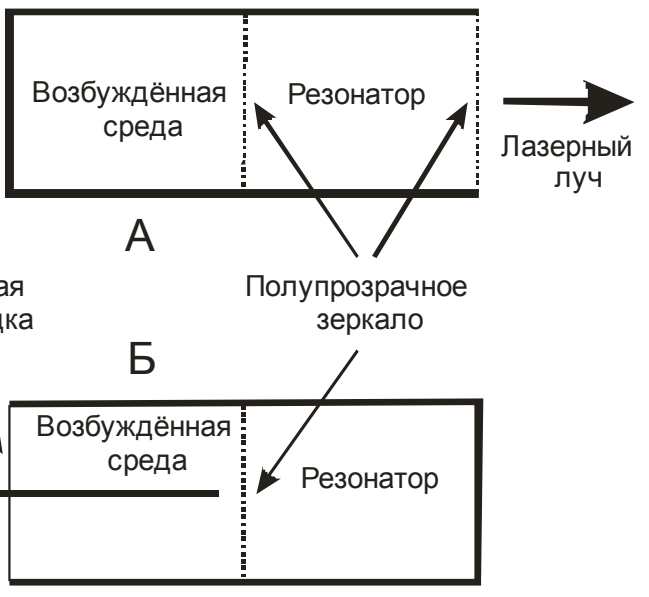

Fig. 55. Schematics of lasers with the active material beyond the limits of the resonator

Two constructions of laser, in which the active material is located beyond the limits of resonator, are represented in Fig. 55. In the version A external resonator has two semi-transparent mirrors: through the left mirror it occurs its wash- 
ing, while laser beam - leaves through the right. From the side of active material on the semi-transparent wall of resonator be incident the waves with the most varied phases and the polarization, but only wave with the polarization and the frequency, that coincide with one of the modes of resonator, and which with the drop on the mirror will prove to be coherent, they will excite in the resonator of fluctuation. These fluctuations, after passing outside through the semitransparent mirror, and form laser beam. In the version B the external resonator is had only one semi-transparent mirror, through which is produced and its washing, and the emergent beam of laser. The constructions indicated are called to finally break myth about the fact that the laser is quantum generator.

The principle of the quantization of action leads to the fundamental, actually, to insoluble problems in contemporary theoretical physics. From this principle it turns out that the energy can change only spasmodically (by portions). Idea about the quanta of energy, which the physical systems are exchanged between themselves hence arose. The fault of this approach lies in the fact that the discontinuity of a change in the energy unavoidably leads to the local (at the level of microcosm) disturbance of the law of conservation of energy even for the conservative systems. Namely, is allowed this disturbance of the law of the conservation of energy of conservative system, with which the value of action does not exceed Planck's constant. Thus, is allowed even an infinitely large increase in the energy, but only during the infinitely small time interval (the greater the increase in the energy, the less the corresponding time interval).

Similarly, as in the general relativity is broken the rule of conservation of energy for the totality of massive material (substance, antimatter and nongravitational fields) and gravitational field, the quantization of action also leads to the specific disturbance of the law of conservation of energy. But this is too great a loss for physics, to accept which without the most direct experimental confirmation is inadmissible.

\section{§ 26. Physical substantiation of Huygens principle and the reciprocity theorem}

The Huygens principle says, that each element of wave front can be examined as the center of the second disturbance, which generates second spherical waves, and the resulting light field at each point of space will be determined by the interference of these waves. This principle is the basic postulate of geometric optics; however, it does not reveal physical nature of this phenomenon. From geometric optics it is known that any beam of light is dispersing and that the area of its section in the process of propagation always increases. This phenomenon is subordinated to Huygens's principle. But is there any physical explanation of this principle? Let us show that this substantiation exists [69].

Let us examine flat monochromatic TEM wave, passing through the slot, whose width is considerably longer more than wavelength (Fig. 56). 


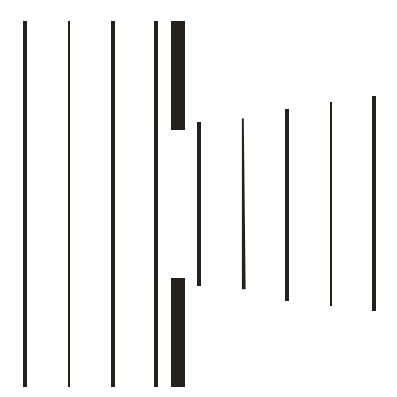

Fig. 56. Passage of the plane wave through the slot

After passage through the slot the wave begins to be enlarged in the transverse direction, and this expansion is subordinated to Huygens principle, when in the expanding wave the ends of the paths of constant phase in the process of their motion in the direction of propagation of wave move with the speed of light still, also, in the transverse direction. But since with this expansion increases beam section, begins to decrease Pointing vector, which indicates the decrease of electrical and magnetic field on the paths of constant phase. This process of the self-expansion of electric vectors on the paths of constant phase is such to the process of the selfexpansion of electriccurent wave in the long line described in the thirteenth paragraph. Difference is only the fact that in the line the wave of transverse electric field is propagated, and self-expansion occurs in the direction of propagation. In the long line there is no such expansion, since wave in the transverse direction I limit the conductors of line. The transverse transformation of wave is accompanied by the fact that, beginning from the center of path of constant phase along it begins leak bias current. This process is very similar to the expansion of the compressed elastic, when all its sections begin evenly to be enlarged. In this case the energy density of electromagnetic wave begins to decrease, being evenly distributed in the increasing volume, occupied by the expanding wave. This simple examination, he indicates the physical causes for Huygens's postulate and is in fact new physical law.

With this phenomenon is connected the so-called reciprocity theorem for the antenna systems, which does not up to now have its physical substantiation. This theorem says, that the mu-factor of the directional antennas is identical both with the emission and with the reception of signal. The strangeness of this theorem consists in the fact that the directional antenna can form the narrowly directed beam, when the radiated energy is concentrated in some direction. This means that the energy density is concentrated in the space limited from the lateral sides. This one can see well based on example of laser beam. However, receiving directional antenna is located in the fields of transmitter, which are evenly distributed in the space, and in order to increase its mu-factor to the directional receiving antenna it is necessary to know how to gather energy from the lateral space. This actually so, but as this it makes, until now, it remains riddle. A question does consist in that, is it possible to find some physical causes for this strange behavior of the directional receiving antennas. 
Is known that the laws of geometric optics, when ray can be considered practically rectilinear, work when beam width considerably more than wavelength. In this case Huygens principle works. Therefore, if we limit the width of beam with the aid of the slot, then its divergence will begin grow, and when the width of slot will become commensurate with the wavelength, after the slot we will obtain the strongly divergent ray, and when slot will become less than the wavelength, then after slot we will obtain the radially divergent waves.

However that will be, if we on the way of transverse wave place obstacle? Let us examine two cases. In Fig. 57 is depicted the case, when the width of obstacle is considerably lower than the wavelength

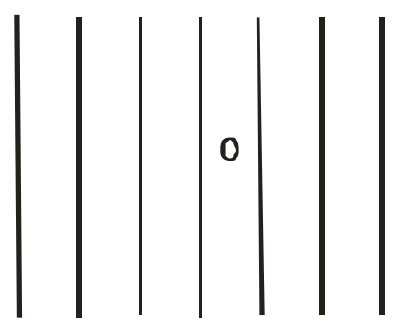

Fig. 57. Diffraction by the waves of the obstacle, whose dimensions are considerably lower than the wavelength

Practically it they do not feel with the diffraction of this obstacle of wave.

We see other entirely picture, when obstacle is considerably more than wavelength (Fig.58)

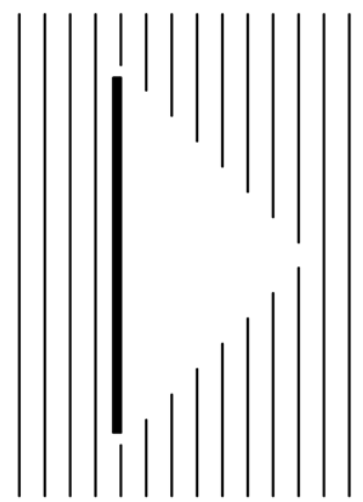

Fig. 58. The case, when on the oscillation loop is located the obstacle, whose dimensions are greater wavelength

If obstacle on the oscillation loop is executed so that it completely absorbs energy of the incident to it waves, then the picture of wave process appears in the manner that shown in Fig. 56. It is evident that from the right side from the absorbing obstacle there is a shadow zone, where the waves be absent. But passing obstacle, the ragged ends of the waves in accordance with Huygens principle again begin to converge, bringing additional energy into the space of shadow from the lateral space, which is located beyond the limits of the possible arrangement of the following elements of the directional antenna. This phenomenon bears the name of diffraction. And it is characteristic for any wave processes, including for the elec- 
tromagnetic waves. But this process has one special feature, which is obvious. Since the absorbing obstacle absorbed the part of wave energy, the wave amplitude to the right of shadow, will be somewhat less than wave amplitude to the left of the absorbing obstacle. This being connected with the fact that the sections of the waves to the right of obstacle, enlarged, they redistribute their energy in the shady section of space after the obstacle. Direction the motions of energy in the expanding section of wave are shown by pointers. This means that in order to liquidate shadow and to restore normal wave process, wave energy begins to be pumped over from the lateral sections of those removed from the shadow zone, enlarging the sections of waves torn by obstacle.

Let us return to the receiving directional Yagi antenna and let us assume that the second director we arranged out of the shadow in that place, where electric fields arrived, after bringing additional energy from the lateral regions. Situation in this case will be repeated, again shadow is formed after this director. The third director we also can arrange beyond the limits of the shadow of the second director, etc. And each time each new director will occur in the fresh electric fields, taking away in them the energy assumed to him.

But situation with the real directed antenna of the type wave duct is somewhat another. First, the length of director is equal to half of wavelength, and after it practically there is no shadow and the ragged pieces of wave practically immediately are clamped after the director, but in this case energy transfer from the lateral sections into the region of the arrangement of the following director nevertheless occurs.

Let us examine, as behaves this antenna in the field by the flat TEM wave (Fig. 59). The let us assume that numbered wave consecutively occupies positions 1,2 , 3 and 4. After flying so far to the first director, it excites in it currents, making with its emitter, but in this case the part of its energy loses. In this case the first director re-emits the obtained from the wave energy into that surrounding space correspondence with his radiation pattern. Therefore wave after the passage of the first director directly in the region after it has the smaller amplitude of electric field, than to its passage.

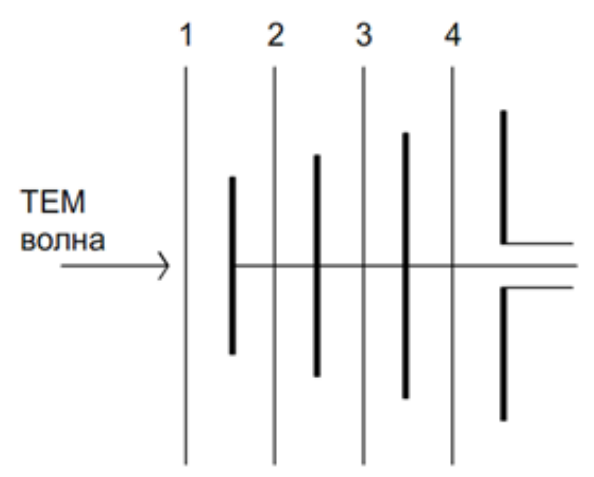

Fig. 59. The Yagi antenna in the field of TEM of the wave 
In order to compensate for the losses indicated energy from the lateral sections, which lie beyond the limits of antenna field, will begin to be pumped over to the side of the axis of antenna. After the passage of the second director will occur the same. Thus, the phenomenon of diffraction will lead to the fact that the wave energy from the sections beyond the limits of antenna field will begin to be pumped over into the region of finding the directors. But the currents, induced in the directors, will make the active vibrators, which will increase currents in each subsequent director and after reaching the pick-up dipole, of them, these fields will considerably exceed the fields of wave itself in position 1 . With this is connected the circumstance that the directional receiving antenna possesses larger effectiveness than single vibrator. Although to, of course, assert that the mu-factors, both in the regime of transfer and in the reception mode they will be identical, are cannot. Therefore reciprocity theorem although is carried out, most likely, not fully.

\section{$\S 27$. New system of units}

Is well known the word: mass, space, time. However, in the literary sources there is no precision determination of these concepts. If we speak about the mass, then to us, at least, are known its three properties, which it characterize as mass. The first property consists in the fact that any mass has linear dimensions. If it did not have this property, then it would be unobservable. The mass is had two additional fundamental properties, in connection with which to be introduced this concept as force. The law of universal gravitation indicates this concept. Actually, two masses are always attracted in accordance with this law. This property is the consequence of the fact that around the mass is observed the potential field, whose gradient is critical for the appearance of this force. This also means that the system of two remote bodies possesses potential energy. The third fundamental property of mass is this its inertia properties, which indicate that for accelerating the mass, it is necessary to exert force. From this property escapes the fact that the moving mass possesses kinetic energy. Thus, mass as physical concept possesses the following fundamental properties: it has linear dimensions, and also it can possess potential and kinetic energy.

The concept of space with the concept of linear dimensions or length, space is connected three-dimensional. Coordinate systems are introduced for the formal realization of this concept. But the space has one additional characteristic, which can be named exclusion principle. This principle consists in the fact that at one and the same point of space at the given instant, cannot be located two different masses. Strictly this principle defines one of the characteristics of this concept as time, that attests to the fact that the different bodies simultaneously can be located only at the different points of space.

What is time as why it flows, scientists philosophers argue to the these rapids. It is known that time on the level with the mass and the length, enters into all systems of units as primary not on what the not depending value. However, it is known that, in order to measure the time, hours are necessary. There are many types of different hours, but all them unites one special feature. It occurs that in all conceiv- 
able hours, always occurs interaction of other primary physical quantities, after all as masses, length and force. In the pendulum hours their motion is determined by the mass of the Earth and by the length of pendulum. The same relates also to the satellites, which revolve around their stars or planets. In the hours with the mechanical springs the motion is determined by mass and dimensions of pendulum, and also by elastic properties of spring. Mechanical resonance systems can be used as the hours, but also in the required order here occurs interaction of three primary parameters: force, mass and length. Electromagnetic resonance systems also can be used as the hours, but also here their motion will depend on the dimensions of resonator, and also on the dielectric and magnetic properties of medium.

But give let us visualize that in this inertial system suddenly for some reasons changed the gravitational constant, either the inertia properties of mass changed, or the electrodynamic properties of medium finally changed - all this will involve a change in the rate of the motion of hours. Thus, asserts itself the conclusion that time is not primary physical quantity as, for example, the mass length and force, but directly it depends on the values indicated it can be through them expressed [70, 71].

Important is a question about side to what, and as rapidly flows the time. It is known that practically all laws of microcosm are invariant with respect to sign change of time; therefore for these laws does not have a value to what side it flows time.

If we have a frame of reference, which passes of one inertial system to another, which is unavoidably connected with the processes of retarding or accelerating this system, then in this system the process of retarding or accelerating the time must occur. Thus, it is possible to consider that the time can leak unevenly, first being accelerated, then slowing down. But if this is so, that arises question, and time can generally stop, or change its direction. Almost obvious it is the fact that, if any motion suddenly ceased, and all bodies, including atoms, suddenly they stood still at its places, then the concept of time would lose its sense. The same would occur when the universe was absolutely empty. Thus, asserts itself the conclusion that the concept of time is the consequence of existence of material objects and their properties.

Is known that time reversal, i.e., sign change of time does not change the form of equations of motion. This means that for any possible motion of system can be achieved the time-reversed motion, when system consecutively passes to the reverse order of the states, symmetrical to states, passed in the previous motion. In this posing of the question naturally to assume that, when in the system it occurs no changes, then time for this system not at all flows. When in the system some reversible changes occur, i.e., it after a certain evolution returns reversibly to its initial state, the time flows first in one, and then in other direction. Since in this case the concept of time is used to in application to this concrete system, it is possible to introduce the proper time of system, i.e., to assume that in each separately undertaken system there is its proper time. States symmetrical on the time are characterized by opposite directions of the speeds (pulses) of particles and magnetic field. Temporary invariance leads to specific ratios between the probabilities of direct 
and reverse reactions, to the prohibition of some states of the polarization of particles in the reactions, to the equality to zero electrical dipole moment of elementary particles and T. d. It follows from the general principles of the quantum field theory that all processes in nature are symmetrical relative to the work of three operations: the time reversal, three-dimensional inversion and charge conjugation.

However the existing systems of units do not assume the application of time with the different signs. Why thus it did happen? Most likely because the time as physical quantity was introduced not on the basis any deep physical principles, but on the basis the solutions of the chamber of measures and weights. Simply for measuring the time were undertaken the existing in nature periodic processes, which frequently have different nature.

As was said, the hours, with the aid of which the time is measured, compulsorily operates with other physical quantities, such, for example, as mass, length and force. And if we express time through these parameters, then their combination will prove to be under the root, and it means and time will be able to assume both positive and negative values. But, although mass, length and force exist as the primary objectively existing physical quantities, we will encounter that difficulty, that by the existing systems of units force itself is evinced after the already introduced time. Is there a way of overcoming this difficulty? Yes, this way is located.

The mass itself in accordance with the law of universal gravitation is the carrier of force, since. two masses, spread in the space, are attracted. It is known from other side that there is a principle of the equivalence of heavy (gravitational) and inert mass. Moreover it is experimentally proven that this principle is observed with the very high degree of accuracy. Specifically, these two principles can be undertaken as the fundamental bases for the introduction to time as physical quantity.

If are located two identical masses $m$, located at a distance $2 r$, then, in accordance with the law of universal gravitation, the force of their attraction determines the dependence:

$$
F_{g}=\frac{m m}{4 r^{2}} .
$$

We will not thus far introduce any conversion factors, since. it is built new system of units. It will be shown below, what it is necessary to use conversion factors in order to switch over to customary us to the units of time.

If the masses indicated revolve around the overall center of masses and acts the principle of the equivalence of gravitational and inert mass, then the equality will be carried out:

$$
T=4 \pi \sqrt{\frac{r^{3}}{m}}
$$

where $T$ - period of revolution of masses around the overall center.

The relationship (28.1) includes immediately two laws: the law of universal gravitation and the principle of the equivalence of gravitational and inert mass. It also determines the dimensionality of time. Certainly, this dimensionality to us is unusual, but became accustomed we to other dimensionality in physics, into which 
enters incomprehensibly from where undertaken second. The advantage of this approach is the fact that the time as physical quantity is introduced on the basis of the fundamental laws of physics and it, as a consequence of this, corresponds to the principle of time reversal.

If we as the unit of length take meter, and as the unit of mass - kilogram, the unit of time in this system will be the value $4 \pi$. The dimensionality of time in this case follows from relationship (28.1) of in order to transfer this value into seconds, should be divided it into square root of gravitational constant. If we this make, then let us see, that the newly introduced unit of time is approximately five orders more than second. This, of course, is not very convenient, but in order to avoid these inconveniences, it is possible to introduce the dimensionless coefficient, equal to square root of the absolute value of gravitational constant. In this case the relationships between the values of all physical quantities will be preserved, although the dimensionality in them will be others. All mechanical values in this case will be expressed only through the length and the mass.

Since time now has its own dimensionality, passage to the electrical systems of units also does not compose labor, simply into the appropriate dimensionality of ones it is necessary to put the new dimensionality of time with the selected dimensionless conversion factor. If we for measuring the electrical units use to Gauss a system and to express in it time in the units of mass and length, then all electrical and magnetic units will be also expressed in the units of mass and length.

It should also be noted that the adoption of this innovation can lead to serious reconstruction of our views.

\section{$\S 28$. The liquid-drop model of electron and atom}

In the article is examined the liquid-drop model of electron and atom, which assumes existence of electron both in the form the ball-shaped formation and in the form liquid [72-75]. This model is built on the same principles, on which was built the liquid-drop model of nucleus, proposed by Bohr and Weizsacker. Phenomenon of the electrization of dielectrics known long ago. With the friction the dielectrics acquire booster charge, in this case the electrons pass from the dielectrics with the smaller dielectric constant to the dielectric, whose dielectric constant is more. Millikan established that with the dispersion in air of oil of his drop the discrete charges acquire. This made it possible to make the conclusion that the charges can have only discrete variable and the measured discrete magnitude of the charge of drops was defined as electron charge. Experience does not give the possibility to establish, from where drops obtained discrete charges. These charges could be obtained with the transformation of oil into the drops in the process of its dispersion.

The discrete charge of drop they could obtain also with interaction with the nozzle of atomized-spray injector, or in the process of interaction with atmospheric air. In air be contained to always vapors of water, and since the dielectric constant of water is great, i.e. molecules could take away charges in the drops of oil. As a result these experiments electron began to consider ball-shaped formation with the specific sizes and the discrete charge. Since was established that the electron has 
discrete charge and has ball-shaped form, became a question about the special features of its presence in the constitution of atom. The idea of the Bohr orbits of electron in the atom thus was born. This idea assumes that the electrons revolve around the positively charged nucleus, being found in specific orbits. Passage from one orbit to another is accompanied by the emission of the quanta of the electromagnetic radiation, when each quantum bears the specific bundle of energy. These assumptions became the basis of quantum mechanics. But in this model there exist the contradictions, which are not removed to the these rapids. With its electron motion must continuously emit electromagnetic waves, but, moving in the constitution of atom, it does not emit. In addition to this the simplest atom of hydrogen, which consists of the proton and the electron revolving around it, must have magnetic moment, but hydrogen atom of this moment does not have. We must conclude for this reason that the physically substantiated model of the simplest atom, which is hydrogen atom, until there exists.

But problems are located not only with electron and atom of hydrogen. Is not clear nature of structure and proton, or complex nuclei, in which act nuclear forces. It proposed the liquid-drop model of nuclear structure in 1936. Boron in order to explain the long times of life of the excited nuclei of the heavy elements, the generatrix during capture of the slow neutrons [72]. It developed Weizsacker, considering nucleus as the spherical drop of incompressible charged nuclear fluid [73]. The proposed model had large haste, and with its aid it was possible to explain many properties of nuclei and to, in the first place, obtain semi-empirical formula for the nuclear binding energy.

The electron can be found in the bound state in the constitution of atom, and also in the free state in the form of electron beams or near the incandescent cathode in the electronic devices. In the free state electron to be found also in the conductors, when it can freely be moved into the tele-conductor. But if we consider electron the ball-shaped formation of the specific sizes, then problems here appear. In the superconductive state the depth of penetration of magnetic fields on and currents composes values the strand of several hundred angstroms, while the value of the surface roughness it is measured by microns. The electron velocity in superconductive niobium with the critical magnetic field is about $300 \mathrm{~m} / \mathrm{s}$. If electron was ball, then moving along so twisting a trajectory, it due to the inertial forces would destroy surface, but this it does not occur. Therefore possible being to assume that located in the composition of conductors, electrons present liquid, and they move according to its laws. When conductor they warm to the high temperature, this liquid similar to water vapor evaporates from the surface of conductor. After exceeding the limits of conductor, vapors of this liquid are condensed into the drops, forming electrons.

Liquid has the surface tension, because of which the drop of liquid acquires ballshaped form. In this case internally the pressure in the drop is created by the forces of surface tension, which act on the surface. The pressure, created by the surface of drop is determined by the relationship

$$
p_{\sigma}=\frac{2 \sigma}{r},
$$


where $\sigma$ - coefficient of surface tension, $r$ - radius of drop.

The electron is had the external electric field, which attempts to tear electron, these force fields on in the direction they are reverse to the forces of surface tension. Their pressure on the surface of electron is determined by the relationship.

$$
p_{E}=\frac{1}{2} \varepsilon_{0} E_{s}^{2},
$$

where $E_{s}$ - tension of electrical fields on the surface of electron. The tension of electrical fields on the surface of electron it is determined by the relationship

$$
E_{s}=\frac{e^{2}}{32 \pi^{2} \varepsilon_{0} r_{e}^{3}}
$$

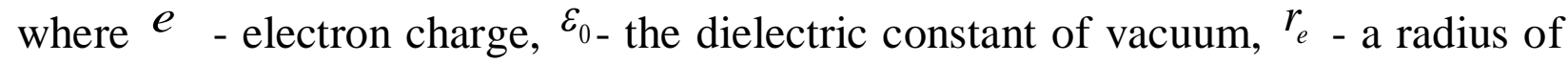
electron. Equalizing relationships (27.1) and (27.2) and taking into account relationship (27.3) we obtain the coefficient of surface tension for the electronic liquid

$$
\sigma_{e}=\frac{e^{2}}{64 \pi^{2} \varepsilon_{0} r_{e}^{3}}
$$

Substituting in relationship (29.4) tabulated data, we obtain $\sigma_{e}=1.5 \times 10^{14} \frac{Д \varkappa}{M^{2}}$.

For the comparison let us point out that for the water the value of surface tension is $73 \mathrm{~J} / \mathrm{m}^{2}$, and for mercury it is equal to $487 \mathrm{~J} / \mathrm{m}^{2}$.

A classical radius of electron composes $2.8 \times 10^{-15} \mathrm{~m}$. Experiments on the measurement of a radius of proton showed that its diameter was equal $9 \times 10^{-16} \mathrm{~m}$.

If we attempt ourselves to place proton inside the electron, then the fields of proton neutralize the charge of electronic liquid, after converting it into the usual badly compressible liquid. Volumetric drop will begin to be enlarged, being converted into the shell (Fig. 60) of This shell will be extended until sets in the equilibrium between the electric forces, which attempt to press sphere and to the elastic forces of the electronic liquid, which prevent this compression. This process will determine the radius of the atom of hydrogen, which is equal $5.3 \times 10^{-11} \mathrm{~m}$. Since the charge of electronic liquid is equal to the charge of proton, electric fields outside the atom will be absent.

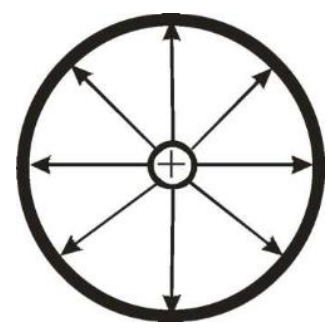

Fig. 60. The liquid-drop model of atom 
Sufficient conductivity of normal metals extended by theory the Drude model appears. Electrons in the metal are considered as electron gas, to which it is possible to apply kinetic theory of gases. It is considered that the electrons, as the atoms of gas in the kinetic theory, are the identical solid spheres, which move along the straight lines until they encounter with each other. It is assumed that the duration of separate collision is negligible, and that between the atoms it acts no other forces, except the forces, which appear at the moment of collision. Since electron is negatively charged particle, then for observing the condition of electro neutrality in the solid tele- also must be the particles of another type, i.e., the positively charged ions. Drude assumed that the compensating positive charge belongs to the ions, which it considered fixed.

Despite the fact that gas density of conduction electrons is approximately 1000 times more than the density of classical gas at normal to temperature and pressure, in the Drude model the methods of the kinetic theory of the inert rarefied gases adapt. The basic assumptions of the theory of the Drude consist of the following:

In the interval between the collisions is not considered interaction of electron other electrons and ions even it is considered that each electron moves with the constant velocity along the straight line. Further, it is considered that in the presence of external fields on electron it moves in accordance with Newton's law. In the Drude model, as in the kinetic theory, collisions are the instantaneous events, which suddenly change the electron velocity, and time between two sequential collisions of is called relaxation time. This time enters into the relationship, which determines the conductivity of the metal

$$
\sigma=\frac{n e^{2} \tau}{m} .
$$

In this case the connection between the current density in the metal and the tension of electric field takes the form:

$$
\mathbf{j}=\sigma \mathbf{E} .
$$

It is assumed that the electrons come into the state of thermal equilibrium with the lattice exclusively because of the collisions.

The theory of the Drude satisfactorily describes the phenomenon of the conductivity of metals and up to now successfully it is used in the electrodynamics.

The drop theory, when electronic component in the metal is considered as electronic liquid, changes approach to the determination of the conductivity of metal. Task is converted into the hydrodynamic task along the flow around obstacles of the moving liquid. With the flow of the liquid about the fixed obstacles are two regimes: laminar and turbulent. For each form of flow there is critical Reynolds number, which determines passage from the laminar flow to the turbulent. With the fulfillment of conditions $\operatorname{Re} \leq \operatorname{Re}_{c r}$ occurs laminar flow, with $\operatorname{Re} \geq \operatorname{Re}_{c r}$ in the liquid appear turbulences. With the laminar flow of liquid energy losses be absent, and, therefore, is absent resistance. In the turbulent regime, with the diffraction of obstacles in the liquid appear turbulences, which lead to the energy losses. Specifically, by this it is possible to explain the fact that even at temperatures, which are approached absolute zero, the end resistance is observed in metals. But if the obstacles streamlined with liquid accomplish oscillatory or other motions, then this 
leads to additional turbulences, and, therefore, also to an increase in the resistance. And the greater the amplitude of the fluctuations of the streamlined obstacles, the greater the resistance. This circumstance leads to the dependence of the resistance of metals on the temperature, since. with an increase in the temperature the amplitude of the oscillations of lattice ions increases.

The approach examined can be used for explaining this phenomenon as the superconductivity, which can be the consequence of the passage of the flow of electronic liquid from the turbulent to the laminar.

Superconductors have the critical temperature, lower than which they convert to the superconductive state. This means that with the amplitudes of the oscillations of lattice ions of superconductor the laminar possible flow of electronic liquid is lower than the certain critical value. In the superconductors of the second kind there is a phase of the mixed state, when vortex formations can be created with the way of the imposition of external magnetic field. In this case Abrikosov vortices are formed. With the flow of electronic liquid these vortices begin to move, which leads to the appearance of resistance. The case, when in connection with the presence of the defects of crystal lattice, vortices are attached on such defects, is exponential, in this case the vortices cannot move, and resistance is absent. In the usual hydrodynamics this situation is realized be it cannot.

The liquid-drop model of atom examined transfers a question about the presence of resonances in the atom into the mechanical task. If there is an elastic shell, then it has the infinite number of mechanical resonances. These resonances can be to bear the axial nature, when standing wave has axial symmetry. Are possible also the resonances, when the integer of half-waves is plotted along the equator of sphere. But this system will possess still one type of the fluctuations, which generate the circularly polarized electromagnetic radiation. During the collision with other it will pass the displacement of electron shell on the relation to the nucleus as atoms. As a result this is formed the being varied and simultaneously revolving electric dipole. The emission of this dipole will be received by receiver as the emission of the specific frequency, modulated in the amplitude, and which, therefore, contains the carrier frequency and side frequencies. The totality of such frequencies will compose the radiation spectrum of atom.

The proposed liquid-drop model of electron and atom this thus far only hypothesis, but it has right to existence as the liquid-drop model of nucleus. We attempted to describe only very idea of drop approach to the circumscription of electron, further development of these ideas in addition to of the liquid-drop model of nucleus can lead to the creation of the generalized liquid-drop model of atom.

\section{§ 29. Gravitational mass defect}

Let us examine one phenomenon, which refers straight to the case of liberating the large quantities of energy [76]. Let us take the case, when trial body with the mass $m$ falls on the very massive body with the mass $M$, whose radius is equal $R$ (subsequently the body $m$ and the body $M$ ). Let us assume that at the initial moment of time the distance between the bodies is very great and that is fulfilled the 
relationship $\quad M>>m$. We assume to also consider that the density of the massive body $\rho$. The rate of the fall of the body $m$ on the body surface $M$ in this case can be found from the relationship:

$$
v=\sqrt{\frac{2 \gamma M}{R}}
$$

where $\gamma$-gravitational constant. If we switch over to substance density of massive body, then relationship (29.1) can be rewritten as follows:

$$
v=2 R \sqrt{\frac{2 \pi \gamma \rho}{R}} .
$$

Is obvious that kinetic energy, which possesses the falling body, it obtained from the gravitational field of the body $M$. This kinetic energy of the falling body with its drop on the surface of massive body to become thermal energy will be radiated into the surrounding space in the form of electromagnetic waves.

From the aforesaid it is possible to conclude that the final summary mass of two bodies will not be equal to the sum of the masses of bodies prior to the beginning of the drop [76]:

$$
M_{\Sigma} \neq M+m,
$$

i.e. there is a gravitational mass defect. The relationship of the honey $M_{\Sigma}$ and $M+m$ can be found, knowing that kinetic energy, which possessed the body $m$ with the drop on the body $M$. This energy can be calculated from the relationship

$$
E_{k}=m c^{2}\left(\left(1-\frac{v^{2}}{c^{2}}\right)^{-1 / 2}-1\right) .
$$

During the record of this expression is taken into account the circumstance that with the fall of body in the gravitational field the acceleration of this body does not depend on its mass. Therefore relationships (29.1) and (29.2) are accurate even for the relativistic speeds. It is now not difficult to calculate gravitational mass defect.

$$
\Delta m=E_{k} / c^{2}=m\left(\left(1-\frac{v^{2}}{c^{2}}\right)^{-1 / 2}-1\right) .
$$

This effect comprises with the drop on the earth's surface $\sim m \times 10^{-9}$.

From relationship (29.3) is evident that the addition $\Delta m$ can be both less and it is more than $m$. If $\Delta m<m$, then with the fall of body summary mass increases. But if $\Delta m=m$, then an increase in the summary mass ceases, and entire mass of the falling body is converted into the thermal radiation. In this case massive body is converted into the ideal anvil, which converts entire mass of the falling body into the energy of electromagnetic radiation. 
As can easily be seen of relationship (29.3), the rate of the fall of the body $m$ (let us name this speed of critical) to the body surface $M$ will be determined by the relationship

$$
v_{\kappa p}=\frac{c \sqrt{3}}{2},
$$

i.e. it is considerably less than the speed of light.

If the density of massive body is known, then, using relationships (29.2) and (29.4), it is not difficult to find a critical radius of this body:

$$
R_{\kappa p}=\frac{3 c}{4 \sqrt{2 \pi \gamma \rho}} .
$$

By this concept we will understand the value of the radius, with reaching of which further increase in the mass of the body $M$ due to the fall on it of another body becomes impossible.

Can occur the situation for the space objects examined, for example for the neutron stars. It is known that the neutron stars (pulsars), have very high density [77]. So pulsar with a mass $\sim 2 \times 10^{30} \mathrm{~kg}$ (mass of the sun) would have a radius a total of near $10 \mathrm{~km}$ of. Its density in this case would compose $\sim 5 \times 10^{17} \mathrm{~kg} / \mathrm{m}$. With this density a critical radius would comprise near $15 \mathrm{~km}$ of; and mass would compose 3.4 of masses of the sun. This means that with reaching of such sizes and this mass the neutron star no longer can increase neither its sizes nor its mass, since any falling to it objects will be completely converted into the radiant energy.

According to preliminary calculations in our galaxy is counted about 300 thousand neutron stars [77]. What to happen, if neutron star does encounter the same neutron star as it itself? It is obvious that the complete annihilation of neutron substance and its transformation into the energy will occur. Taking neutron star with a critical radius $15 \mathrm{~km}$ of. and with the mass $\sim 3.4$ of masses of the sun, we obtain the value of energy $5 \times 10^{47} \mathrm{~J}$. This value of energy is very close to that energy, which characterizes explosion in the nucleus of galaxy NGC 3034 [77]. During this explosion from the nucleus of galaxy was rejected a huge quantity of material throughout its mass equal $6 \times 10^{7}$ of the masses of the sun. This of phenomenon does not find its thus far explanation, since are not known those energy sources, which can lead to so immense an explosion. The process of the collision of neutron stars examined can be precisely such source.

In its essence this explosion - this is the explosion of the nuclear charge of very large power. The isolation of such significant quantities of energy will be accompanied by warming-up and transformation into the plasma of large quantities of surrounding material. This in turn will lead to the appearance of the same electrical fields on as with the explosion of nuclear bomb, only much more significant. The presence of such fields on in the surrounding space they must lead to the appearance of specific polarization effects. To them can be attributed polarization in the electric fields of atoms and molecules and the appearance of the electric dipoles, which will lead to the polarization of the electromagnetic waves of those extending in the plasma. 


\section{§ 30. Three-dimensional coherence}

With the aid of the phased antenna arrays it is possible to create the pencil beams of electromagnetic waves. The process of the formation of such beams is connected with the addition in the space spatially coherent waves. Addition (interference) is accomplished in such a way that in the determinate directions the phases of waves from the separate emitters are added, and in others - they are read. This is the only way of creating the narrowly-directed beams, independent of nature of wave processes and wavelength. Is correct reverse, if we see the narrowly directed beam, for example laser beam, then it is possible to assert that it is formed with the aid of the separate emitters, which emit signals with the large length of coherence and phase of which in the space they are added in those places, where we see ray itself. But unidirectional beams of light can be obtained by simpler way and this we not one time observed. If sunlight was passed through the opening, thus almost rectilinear ray is formed. But indeed sun itself emits monochromatic and far from coherent waves. But in than then the matter? If we make a good hologram with the aid of the laser of that emitting red light and to illuminate by its sunlight, then it is possible to see holographic picture in the red light. This experiment again confirms that in the composition of sunlight is this spectral line with the large length of coherence, since only with the aid of the coherent light it is possible to see hologram. But as so, indeed the surface of the sun emits in no way coherent emission, and for some reason this no coherent radiation from the sun suddenly at large distances becomes coherent. But give to move from the reverse, once is located the narrowly directed beam, which means, it is formed with the aid of the addition of coherent signals. If we with the aid of the opening cut out ray from the distant star, thus will possess still smaller divergence and larger coherence than solar. The same experience can be made by the distant lamp, which is practically point-source radiator, and to obtain from it pencil beam. If we continue these experiments, then it is possible to see that the less the solid angle, at which is visible the source, the greater the coherence of signal it gives, moreover in entire range of the spectrum radiated by it. If we from this signal with the aid of the filter isolate the specific narrow spectrum band, then this ray will in no way differ from laser.

But why this strange special feature possess point luminous sources, nothing it is written about this in the existing literature. But the answer the like of the essence of the matter is very simple. If at the particular point space we observe the narrowly directed beam, then, as we already said, it can be formed only by the way of the additions of coherent components, which give separate emitters. On the sun or the star each atom is emitter, moreover each with the radiation pattern, inherent in dipole source. Being this excited by thermal shocks, atom the specific time emits into the space its oscillation spectrum, until it encounters another atom. But, since such atoms very much phase their emissions are chaotic, will always be located the specific quantity of atoms, the phases of emission of which will coincide into what that remote place, where we see the result of this addition in the form of rectilinear ray. Therefore any point source this is of its kind the laser, which emits spherical polychromatic ray. And already from this ray we can with the aid of the openings 
obtain narrow radial rays, and with the aid of the filters make with their monochromatic.

\section{§ 31. Ferroelectric transformer}

In connection with the fact that the law of magnetic electric and electric magnetic induction is symmetrical, must exist the symmetrical technical solutions. Such solutions are located. For example, with the aid of the revolving magnetic field it is possible to create electric motors. For the same purposes it is possible to use the revolving electric field, and the engines, which use this principle, exist. There exists the transformers witch ferromagnetic slug in which with the aid of the magnetic flux they transfer energy of one winding into another. The symmetry of the laws indicated tells us, that must exist the transformer, whose core will be executed not of the ferromagnetic material, but of the ferroelectric. In the technology the transformers with the ferromagnetic cores widely are used. Their incapacity to work at the high frequencies is a large drawback in such transformers. Is connected this with the large inertness of the processes of the reversal of polarity of transformer core. And in this connection question arises, and is it possible to create the transformer, in which as the core is used not the ferromagnetic material, but ferroelectric. Since the processes of electrical polarization have very small inertia, this transformer could work at the very high frequencies.

Let us examine the possible the schematics of ferroelectric transformer. Into the composition of transformer (Fig. 61) enters the parallel-plate capacitor, between plates of which is placed the cylinder from the ferroelectric with the large dielectric constant. On the cylinder is placed the winding of torus, whose ends are connected to terminals 2. During the supplying to the capacitor of alternating voltage in the cylinder there will be leak polarization currents and the time-varying circulation of magnetic field will arise around the cylinder. This circulation will excite in the torus-shaped winding currents and a variable potential difference will appear on terminals 2 .

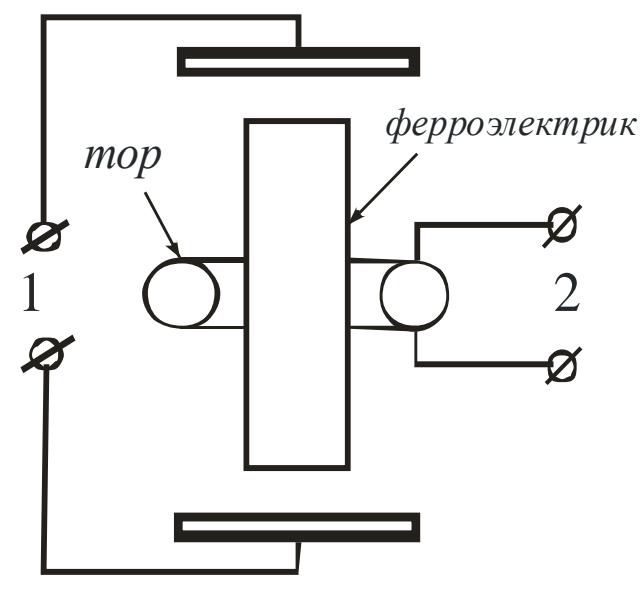

Fig.. 61. The schematic of ferroelectric transformer

The transformer with the torus-shaped ferroelectric core is depicted in Fig. 62. 


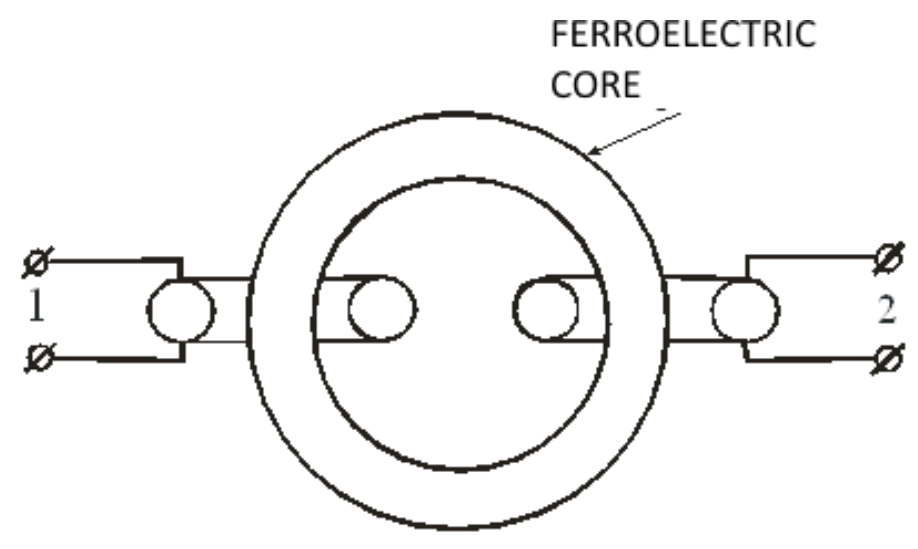

Fig, 62. The transformer with the torus-shaped ferroelectric core is depicted

It consists of the torus-shaped core, made from the ferroelectric, on which are placed two torus-shaped windings. The transformation ratio of this transformer depends on the relationship of the number of turns in the windings. The merit of transformer is the fact that it can work at the very high frequencies. Furthermore, by the way of connection to the windings of the transformer of capacitors it is possible to convert into the resonance transformer.

In spite of simplicity of idea and construction, unfortunately, the transformers of this type before the appearance of works [78] is nowhere described. But indeed they open very large prospects. It is known that the magnetic amplifiers, which possess high reliability, cannot find wide application only because they work at the low frequencies. In this case there are no such limitations in practice, since the processes of electrical polarization have very small inertia, and, using the transformer examined, it is possible to create the reliable wideband amplifiers, which work at the very high frequencies. 


\section{Conclusion}

As a result conducted investigations is established the dependence of the electric field of charge on the parameters of its motion. At the basis of electrodynamics three laws must lie: Coulomb law, the law of the dependence of the electric field of charge on the speed of its motion and the law of the dependence of the electric field of charge on its acceleration. Coulomb law determines the electric field of fixed charge. The presence in the charge of speed leads to increase in its electric field in the direction transverse relative to speed. But the acceleration of charge leads to the induction of additional electric field in the direction longitudinal relative to acceleration. Coulomb law and law of the dependence of the electric field of charge on its acceleration has already been contained in Maxwell's electrodynamics.

However, the law of the dependence of the electric field of charge on the speed of its motion is fundamentally new. The association of all these three laws into the united foundation of electrodynamics radically changes its ideology. It makes it possible to examine from the united positions and static interaction of charges, and the laws of power interaction in the case of their mutual motion, and the emission laws and scattering. This approach made it possible to overcome problems of electrodynamics described in this monograph the theoretical, to explain RS phase aberration and transverse Doppler effect without the attraction, which could not make within the framework a traditional ideology of electrodynamics.

The meaningful result of work - the establishment of the dependence of the scalar potential of electric charge on the speed of its relative motion (concept of scalar-vector potential) and the substantiation of this dependence within the framework of Lagrange formalism. The adoption of this concept is critical, but it is physically transparent, indeed the factor of the motion of charge upon consideration of its influence on the surrounding charges can be reflected in its energy characteristics, so that an increase in the kinetic energy with the acceleration of charge is connected with a change in its electrical pour on.

To experimental confirmation of concept is given in the work significant place. One of them - the appearance of the pulse of electric field with the powerful explosion, so that space nuclear explosions opened at the beginning of the 60 's new page even in fundamental physics.

Pour on Maxwell's equations, which reduce to the wave equations for the electromagnetic, and relationships for power interaction of the current carrying systems with the postulated Lorentz force be the basis of two actually independent divisions of classical electrodynamics. The concept proposed connects them on the united ideological basis.

Are obtained conversions pour on upon transfer of one IRS to another, $v^{2} / c^{2}$ with an accuracy down to the terms coinciding with the relativistic. All experimental data confirm RS not more precise than the order indicated.

It is shown that together with the parameters $\varepsilon \varepsilon_{0}$ and $\mu \mu_{0}$, those characterizing those accumulated or those transferred on medium electrical and magnetic 
energy, exist the two additional fundamental material parameters, the analogously characteristic kinetic and potential energies: kinetic inductance $L_{k}$ and the kinetic capacity $C_{k}$. But only kinetic inductance was used with the description of some physical phenomena, for example, in the superconductors. Use of all four fundamental parameters gives the clear physical picture of the wave and resonance processes, which exist in the material media with the presence in them of electromagnetic pour on, and it makes it possible to solve all existing problems of electrodynamics for the linear media. Electromagnetic waves were considered as the only carriers of energy in the material media that it does not consider all forms of the energy, accumulated and transferred in them by fields and currents. New approach introduces into the examination the magnetoelectrokinetic and electromagnetopotentsial waves, in which the part of the energy is accumulated and is transferred purely mechanically. Resonances in the material media also bear their specific character. In contrast to the electromagnetic resonances in the locked planes, when the energy exchange occurs between the magnetic and electric fields, in the material media there are two forms of resonances. The first electrokinetic, when electric field energy is converted into the kinetic energy of charge carriers and vice versa, but magnetic pour on no generally. The second resonance it is possible to name magnetnopotentsial wave, when the potential energy, accumulated in the precessional motion of magnetic moments, can return into the external space at the frequency of precession.

The physical interpretation of values in the electrodynamics requires caution, since not all they are physical quantities. However, rest are expressed as any physical quantities mathematical parameters, as examples of which it is possible to give the dispersive (depending on the frequency) dielectric and magnetic constants of medium. The phenomenon of the dispersion of electromagnetic waves with their passage through the material media indicates the dependence of phase wave velocity on the frequency. In the conventional interpretation this dependence is generated by the dispersion of the dielectric constant of medium. The more adequate new interpretation, which reveals the mechanism of the formation of this dependence is proposed by the parameters immediately several independent from the frequency. For the plasma the dielectric constant of vacuum and the kinetic inductance of charge carriers, which present plasma, are them. In the dielectrics this process somewhat more complex, because, together with the dielectric constant of vacuum and the kinetic inductance of bound charges, in this process participate the polarization or orientational properties of the electric dipoles, existing in the dielectric. Consequently, the dispersion of electromagnetic waves, which is observed with their propagation in the dielectrics, is connected not with the dispersion of the nonexistent parameter, which was considered the frequency-dependent dielectric constant, but with the dispersion of reactive dielectric conductance. In the formation of this dispersion of conductivity participate the parameters indicated, they on frequency not depending.

The use of that of well being suitable for the case of the dielectrics of polarization vector in the electrodynamics of conducting media at the microscopic level 
limits the adequacy of the corresponding theoretical models, since the charges in the conductors are free and cannot form the electric dipoles. This did not make it possible to reveal new physical phenomenon - the transverse plasma resonance, which can occur in the nonmagnetized confined plasma together with the longitudinal Langmuir resonance, the frequencies in these resonances coinciding, i.e., they are degenerate. But this means that the entire scientific direction, which has great applied value, is passed, since. on its basis can be created the lasers on the collective plasma oscillations, tuned filters, the phenomenon indicated also can be used for the warming-up of plasma. The phenomenon indicated also can be used for the warming-up of plasma.

In the works $[9,17,21,22,80,81]$ a number of the imperfections of the electrodynamics is noted: the presence of exceptions in the Faraday law, the postulation of Lorentz force, the absence of the connection between the Maxwell equations and the description of interaction of the current carrying systems, the introduction of postulates for obtaining the conversions pour on upon transfer of one IRS to another. In the physical plan all these imperfections remove the acknowledgement of the fundamentality of the concept of electric field and the acknowledgement of the dependence of the electric field of charge on the parameters of its motion, making it possible on this basis to build united noncontradictory electrodynamics. Mathematically this is combined with the improvement of the apparatus for differential calculus of field functions in the direction of the possibility to operate with trans-coordinate differential and integral equations.

Many outstanding scientists (amperes, Grossman, Gauss, Lentz, Neumann, Veber, Riemann, Nikolayev, Marinov and other) they subjected the concept of the electromagnetic field of Faraday- Maxwell to doubt. The alternative ideology of electrodynamics proposed in the monograph completely rejects this concept. In contrast to the electric field, the magnetic field does not exist as physical field, but it is the mathematical fiction. Hence it follows that for the complete and noncontradictory description of electromagnetic phenomena one should forego both the postulate about the Lorentz force and Maxwell's equations in any formulation of them, including original in the language of quaternions and idea of Hertz- Heaviside.

One of inherent components of Faraday- Maxwell concept is idea about the electromagnetic waves as the special material substance, which possesses pulse similar to material particles. Therefore the famous experiences of Lebedev in the detection of light pressure convinced then of the validity of Faraday- Maxwell concept thus far of its irreconcilable opponents. But ponderomotive action renders not only wave, but also stationary field. In the monograph it is shown that the ponderomotive action of electromagnetic wave is only a particular manifestation of the more fundamental phenomenon - of power interaction of the current carrying systems, which is obeyed the law momentum conservation. However, the concept about the pressure of electromagnetic wave does not have the definite meaning. Thus, following the failure of the concept of electromagnetic field one should forego also the idea about the presence of pulse in EM waves. 
The concepts of pulse and kinetic energy are not applied to the electric field, as such, and they are applicable only to the material particles and the material bodies formed by them. Thus, there is a fundamental difference between the fields and the particles, so that hardly it is possible to speak about the waveparticle duality as about one of the fundamental physical principles. LagrangeHamiltonian formalism in the field theory is based on the idea of the field in the form of mechanical system with the infinite number of degrees of freedom. But if electric field does not bear pulse, then this idea in the form of mechanical system is deprived of physical sense, but indeed it is the basis of the quantum field theory. The alternative ideology of electrodynamics moreover, proposed in the monograph makes it possible to reveal the nonquantized mechanisms of physical phenomena and technical processes, traditionally considering quantum. Thus, it is shown that the work of quantum generators (lasers) is completely plotted within the framework radio-technical models, if we represent by their phased antenna arrays with the microscopic emitters.

The main result of this work - the substantiation of the long ago ripened need for reorganization not only in the classical electrodynamics with its mathematical apparatus, but also in physics as a whole (including mechanics and physics of microcosm) together with mathematical physics.

The dependence of the electric field of charge on the speed is not sequential physical postulate, but it has more fundamental nature, which far falls outside the limits strictly of electrodynamics. The fundamental physical causes for this dependence indicate the need of constructing new physics, based on the new ideas about the space and the period. In accordance with them the space and time are not the independent, independent forms as in classical nonrelativistic physics, but they are connected with each other into the united space-time. But, it occurs that the space and time are connected not the conversions of Lorenz into the united continuum, but "old good" by the conversions of Galileo into united giperkontinuum. Such ideas about the space and the time can be considered neoclassical in the sense that they return to the conversions of Galileo their fundamental role.

At the basis of giperkontinual physics lie four dynamic laws of conservation: three borrowed from classical nonrelativistic physics (law of conservation of energy, the law of momentum conservation, the law of conservation of momentum of pulse) and the new - law of conservation of kinetic balance, the substituting law of conservation of 4-momentom from the theory of relativity. All four dynamic laws of conservation follow from the variation least-action principle of the under the assumption proper properties of space and time, giperkontinuum connected into the united time-spatial. Just as in classical nonrelativistic physics, from the uniformity of time (invariance of Lagrangian relative to temporary displacements) follows the law of conservation of energy, from the uniformity of space (invariance of Lagrangian relative to three-dimensional translations) - the law of momentum conservation, from the isotropism of space (invariance of Lagrangian with respect to the three-dimensional rotations) - the law of conservation of momentum of pulse. The law of conservation of 4-momentom, which in 
the theory of relativity escaped from the Lorentz invariance of the certificate of space-time (invariance of Lagrangian relative to the Lorenz conversions) in giperkontinual physics it loses its force, being inferior the place for the new law of conservation of the kinetic balance, which escapes from the isotropism of giperkontinuum (invariance of Lagrangian relative to izovelotsitarny, i.e., preserving the module of the velocity vector, the Galileo conversions).

One of the consequences of the law of conservation of kinetic balance is appeared as once the dependence of the scalar potential of charge on the speed. From the same law follows the dependence on the speed of a number of other values, in particular, mass. RS accumulation by the moving body of kinetic energy it is connected with an increase in its mass, but not with a change in the parameters of the created by it physical pour on, that correspond for this accumulation. However, in giperkontinual physics the acceleration of the body with relative changes in all similar connected pour on, including gravitational, whose dynamics is also different from the statics.

Thus, proves to be possible mechanical motion with the faster-than-light speed, is revealed the essential specific character of rotary motion, are opened the prospects of overcoming number of the problems of physics of microcosm, in particular, the local disturbance of the dynamic laws of conservation. Similarly, as the relativistic ideology of electrodynamics it led to the construction of relativistic physics, the proposed in this work alternative ideology of electrodynamics pretends to the role of the initiator of the process of creating new, giperkontinual physics. However, what can prevent putting into theory and practice of such productive ideas? This question has already been discussed in the introduction. Our task, the task of scientists, to bring this matter to the end.

In conclusion let us note still one practically important circumstance. The existing theory of the appearance EMI of nuclear explosion, which is rested on the prevailing ideology of electrodynamics, does not maintain criticism. There were already not justified, although completely substantiated within the framework of this theory of the prediction of Nobel laureate Hans Albrecht Bethe. The new "substantiated" nuclear explosion in space will ruin entire satellite information system, then be justified is late. 


\section{APPRECIATION}

The authors thank professor Henri Amvrosevich Rukhadze and Professor Nikolai Nikolayevich Gorobez for useful advice, which contributed to an improvement in the monograph, and also Nikolai Alexandrovich Drobyshev and Ivan Fedorovich Gorin for the participation in the solution of the separate problems, examined in the monograph. 


\section{СПИСОК ЛИТЕРАТУРЫ}

1. Джемс Клерк Максвелл. Избранные сочинения по теории электрического поля. М.: Государственное издательство технико-теоретической литературы, 1954.

2. П. К. Рашевский. Риманова геометрия и тензорный анализ. М.: Наука, 1967.

3. С. Маринов (Marinov S.) Физическая мысль России 1. 1995. С. 52 - 77.

4. Р. Фейнман, Р. Лейтон, М. Сэндс. Фейнмановские лекции по физике. М: Мир, 1977.

5. F. F. Mende, Problems of Lorentz Force and Its Solution. International Journal of Physics, 2014, Vol. 2, No. 6, p. 211-216.

URL: http://pubs.sciepub.com/ijp/2/6/5

6. М. Фарадей. Экспериментальные исследования по электричеству, в трёх томах. М.: Изд. АН СССР, 1959.

7. М. Фарадей. Избранные работы по электричеству. Москва - Ленинград: Государственное объединённое научно-техническое издательство редакция научно-технической литературы, 1939.

8. Ф. Ф. Менде, А. И. Спицын. Поверхностный импеданс сверхпроводников. Киев: Наукова думка, 1985.

9. Ф. Ф. Менде. Новая электродинамика. Революция в современной физике. Харьков: НТМТ, 2012.

10. Ф. Ф. Менде. К вопросу об уточнении уравнений элетромагнитной индукции. Харьков, депонирована в ВИНИТИ, №774-В88 Деп., 1988, 32c.

11. A. A. Rukhadze, A.F. Alexandrov, L.S. Bogdankevich, Principles of Plasma Electrodynamics, M. URSS, KRASAND 2013, 489 (Second Edition, first Edition, Spriger, Hidelberg, New-York, Tokio 1984).

12. Л. Д. Ландау, Е. М. Лифшиц. Электродинамика сплошных сред. М.: Физматгиз, 1973.

13. В. Л. Гинзбург. Распространение электромагнитных волн в плазме. М.: Наука. 1967.

14. Л. Д. Ландау, Е. М. Лифшиц. Теория поля. М.: Наука, 1988.

15. В. Г. Левич. Курс теоретической физики. М.: Физматгиз, 1962.

16. Л. А. Арцимович. Что каждый физик должен знать о плазме. М.: Атомиздат, 1976.

17. F. F. Mende. Kinetic Induktance Charges and its Role in Classical Electrodynamics. Global Journal of Researches in Engineering, J General Engineering, 2014, Vol. 3, No. 5, p. 51-54.

URL: http://www.engineeringresearch.org/index.php/GJRE/article/view/1260

18. F. F. Mende. On refinement of certain laws of classical electrodynamics. arXiv, physics/0402084.

19. Ф. Ф. Менде. Роль и место кинетической индуктивности зарядов в классической электродинамике. Инженерная физика, 2012, №11, с. 10-19. 20. Ф. Ф. Менде. Новые подходы в современной классической электродинамике. Часть I. Инженерная физика, 2013, №1, с. 35-49.20. Ф. Ф. Менде, 
Кинетическая индуктивность и её роль в электродинамике. LAP LAMBERT Academic Publishing, 2013.

21. F. F. Mende. What is Not Taken into Account and they Did Not Notice Ampere, Faraday, Maxwell, Heaviside and Hertz. AASCIT Journal of Physics, 2015, Vol.1, No. 1, p. 28-52.

URL: http://www.aascit.org/journal/archive2?journalId=977\&paperId=1653

22. Ф. Ф. Менде, Проблемы современной физики и пути их решения. PALMARIUM Academic Publishing, 2010.

23. F. F. Mende. Concept of the dispersion of electric and magnetic inductivities and its physical interpretation. Global Journal of Researches in Engineering: A Mechanical and Mechanics Engineering, 2014, Vol. 14, No. 8, p.11-18. URL: https://globaljournals.org/GJRE_Volume14/3-Concept-of-the-Dispersion. 24. F. F. Mende Conception of the scalar-vector potential in contemporary electrodynamics, arXiv.org/abs/physics/0506083.

25. F. London, Superfluids. Vol.1. Microscopic theory of superconductivity. Nev York: Dower publ., 1950.

26. F. F. Mende. Transversal plasma resonance in a nonmagnetized plasma and possibilities of practical employment of it. arXiv, physics/0506081.

27. F. F. Mende, Transverse Plasma Resonans Mode in an Nonmagnetized Plasma and Its Practical Applications. American Journal of Electrical and Electronic. Engineering, 2014, Vol. 2, No. 5, p. 152-158.

URL: http://pubs.sciepub.com/ajeee/2/5/3

28. А. Ярив. Квантовая электродинамика и нелинейная оптика. М.: Сов. радио, 1973.

29. Ф. Ф. Менде. Кинетическая электрическая ёмкость. Инженерная физика, 2013, №3, с. 49-51.

30. F. F. Mende. Symmetrization and the Modification of the Equations of Induction and Material Equations of Maxwell. AASCIT Journal of Physics, 2015, Vol.1, No. 3, p. 171-179.

URL: http://www.aascit.org/journal/archive2?journalId=977\&paperId=2196

31. В. В. Никольский, Т. И. Никольская. Электродинамика и распространение радиоволн. М.: Наука, 1989.

32. F. F. Mende. Updated Electrodynamics. AASCIT Journal of Physics, 2015, Vol.1, No. 3, p. 149-170.

URL: http://www.aascit.org/journal/archive2?journalId=977\& paperId=2187 33. Ф. Ф. Менде. Существуют ли ошибки в современной физике. Харьков: Константа, 2003.

34. F. F. Mende, New Properties of Reactive Elements and the Problem of Propagation of Electrical Signals in Long Lines. American Journal of Electrical and Electronic Engineering, 2014, Vol. 2, No. 5, p. 141-145.

URL: http://pubs.sciepub.com/ajeee/2/5/1

35. F. F. Mende. Induction and Parametric Properties of Radio-Technical Elements and Lines and Property of Charges and Their Flows. AASCIT Journal of Physics, 2015, Vol.1, No. 3, p. 124-134.

URL: http://www.aascit.org/journal/archive2?journalId=977\&paperId=2144 
36. F. F. Mende. New Properties of Reactive Elements, Lines of Transmission of Energy and the Relaxation Properties of Electronic Fluxes and Conductors. AASCIT Journal of Physics, 2015, Vol.1, No. 3, p. 190-200.

URL: http://www.aascit.org/journal/archive2?journalId=977\&paperId=2262 37. 150 лет волн Герца, Академия наук СССР, 1938.

38. Ф. Ф. Менде. Как скорость удлинения длинной линии влияет на её входное сопротивление. Инженерная физика, 2015, №12, с. 3-5.

39. Ф. Ф. Менде. Великие заблуждения и ошибки физиков XIX-XX столетий. Революция в современной физике. Харьков: НТМТ, 2010.

40. Ф. Ф. Менде. Новые подходы в современной классической электродинамике, Часть II. Инженерная физика, 2013, №2, с. 3-17.

41. F. F. Mende, Concept of Scalar-Vector Potential in the Contemporary Electrodynamic, Problem of Homopolar Induction and Its Solution. International Journal of Physics, 2014, Vol. 2, No. 6, p. 202-210.

URL:http://pubs.sciepub.com/ijp/2/6/4

42. F. F. Mende, Consideration and the Refinement of Some Laws and Concepts of Classical Electrodynamics and New Ideas in Modern Electrodynamics. International Journal of Physics, 2014, Vol. 2, No. 8, p. 231-263.

URL: http://pubs.sciepub.com/ijp/2/6/8

43. F. F. Mende. Concept of Scalar-Vector Potential and Its Experimental Confirmation. AASCIT Journal of Physics, 2015, Vol.1, No. 3, p. 135-148.

URL: http://www.aascit.org/journal/archive2?journalId=977\& paperId=2176 44. F. F. Mende. Classical Relativistic Corrections to Coulomb Law. AASCIT Journal of Physics, 2015, Vol.1, No. 2, p. 69-75.

URL: http://www.aascit.org/journal/archive2?journalId=977\&paperId=1924 45. F. F. Mende. The Classical Conversions of Electromagnetic Fields on Their Consequences. AASCIT Journal of Physics, 2015, Vol.1, No. 1, p. 11-18.

URL: http://www.aascit.org/journal/archive2?journalId=977\&paperId=1647

46. В. Босс. Уравнения математической физики. М.: ЛИБРОКОМ, 2009.

47. А. С. Дубровин. От эталонной модели защищенной автоматизированной системы к общей теории пространства-времени. Вестник Воронежского института высоких технологий. 2010. № 7. с. 37-41.

48. А. С. Дубровин, А.В. Скрыпников, Т.В. Лютова, Е.В. Чернышова, Е.В. Глазкова. Создание эталонной модели защищенной автоматизированной системы в контексте смены естественнонаучных парадигм. Современные проблемы науки и образования. 2015. № 1.

URL: www.science-education.ru/121-18620.

49. А.С. Дубровин, С.Ю. Хабибулина. Пространство-время и информатика: от критики континуума до критики принципа геометризации. Фундаментальные исследования. 2014. № 6. Часть 4, с. 714-718.

50. F. F. Mende. Mechanical and Thermal Electrization Metal, Dielectrics and Plasma. International Journal of Modern Physics and Application. 2015. Vol. 2. № 6. p. 73-99. 
51. А. С. Дубровин. Транскоординатная электродинамика в пространственно-временном гиперконтинууме. Международный журнал прикладных и фундаментальных исследований, 2015. № 12. с. 34-41.

52. А. С. Дубровин. Алгебраические свойства функций одномерных синусоидальных волн и пространство-время. Вестник Воронежского государственного университета. Физика. Математика, 2013. № 1. с. 5-19.

53. А. С. Дубровин, А. В. Скрыпников, Т. В. Лютова, Е. В. Глазкова, Е. В. Чернышова. Общенаучные итоги создания эталонной модели защищенной автоматизированной системы. Фундаментальные исследования. 2015. № 2. Часть 15. с. 3247-3251.

54. A. S. Dubrovin Application of the principle of hierarchy in computer science to representations about space-time in the theoretical physics. International Journal of Applied And Fundamental Research. 2014. № 1.

URL: www.science-sd.com/456-24490.

55. А. С. Дубровин. Модели и методы комплексного обеспечения надежности информационных процессов в системах критического применения: дис. докт. техн. наук. Воронеж, 2011. с. 433.

56. Дж. Джексон. Классическая электродинамика. М.: Мир, 1965.

57. К. Шимони. Теоретическая электротехника. М.: Мир, 1964.

58. А. С. Дубровин. Преобразования Менде в транскоординатной электродинамике. Международный журнал прикладных и фундаментальных исследований, 2015. № 12. с. 1006-1012.

59. Ф. Ф. Менде. К вопросу о возникновении вторичных электрических полей при протекании через сверхпроводники постоянных токов. Харьков, 1992. - 28 с. № 3182-В92. Деп.

60. F. F. Mende. Experimental corroboration and theoretical interpretation of dependence of charge value on DC flow velocity through superconductors. Proceedings International Conference Physics in Ukraine, Kiev, 1993.

61. Знакомый и незнакомый Зельдович (в воспоминаниях друзей, коллег, учеников). М: Наука, 1993, (под ред. С. С. Герштейна и Р.А. Сюняева).

62. Б.В. Болт. В глубинах Земли: о чем рассказывают землетрясения. М., 1984.

63. Дж. Гир, Х. Шах. Зыбкая твердь. М., Мир, 1988.

64. Х. Гупта, Б. Растоги. Плотины и землетрясения. М., Мир, 1979.

65. Н. В. Короновский. Общая геология. Изд-во Московского ун-та, 2002.

66. F. F. Mende. Electrodynamics and thermodynamics of nuclear explosions and TNT. LAP LAMBERT Academic Publishing, 2014.

67. Ф. Ф. Менде. Электрополевая спектроскопия. Инженерная физика, № 9 , 2012, c. $16-18$.

68. F. F. Mende. Electro Spectroscopy of Materials and Samples. Journal of Materials Sciences and Applications 2015, Vol.1, No. 2, p. 70-77.

69. F. F. Mende, Physical Substantiation of Huygens Principle and the Reciprocity Theorem. American Journal of Electrical and Electronic Engineering, vol. 2, no. 6 (2014): 165-170.

URL: http://pubs.sciepub.com/ajeee/2/6/2 
70. F. F. Mende. Material Space Motion Time - New Ideas and the Practical Results, AASCIT Journal of Physics, Vol.1, No. 4, Publication Date: July 7, 2015, Page: 222-228.

URL: http://www.aascit.org/journal/archive2?journalId=977\&paperId=2306

71. F. F. Mende. Material Space Motion Time Phenomenon of Kinetic Energy and Inertia of Material Bodies, AASCIT Journal of Physics, Vol.1, No. 4, Publication Date: July 13, 2015, Page: 292-296.

URL: http://www.aascit.org/journal/archive2?journalId=977\&paperId=2185

72. Н. Бор. Захват нейтрона и строение ядра. УФН, Том 4, № 4, 1936.

73. К. М. Мухин. Экспериментальная ядерная физика. Москва: Энергоатомиздат, 1993.

74.Ф. Ф. Менде. Капельная модель электрона и атома. Инженерная физика, №3, 2015, c. 15-16.

URL: http://infiz.tgizd.ru/ru/arhiv/13754

75. F. F. Mende. Liquid-Drop Model of Electron and Atom, AASCIT Journal of Physics, Vol.1, No. 2, Publication Date: May 6, 2015, Page: 107-110

URL: http://www.aascit.org/journal/archive2?journalId=977\& paperId=1787

76. F. F. Mende, Gravitational mass defect. International Journal of Physics. 2015, Vol. 3, No. 1, 29-31.

URL: http://pubs.sciepub.com/ijp/3/1/5

77. Т. А. Агекян. Звёзды, галактики, Метагалактика. - М.: Наука, Главная редакция физико-математической литературы, 1981.

78. Ф. Ф. Менде Ферроэлектрический трансформатор. Инженерная физика, №4, 2012, c. 15-16.

URL: http://infiz.tgizd.ru/ru/arhiv/9799 


\section{Review}

to the monograph of Mende F. F. Dubrovin A. S. «Alternative ideology of electrodynamics", 2016, 210 p., Fig. 62 , ref. 78

The authors present the alternative electrodynamics, in which instead of the concepts of the tensor of complex dielectric constant (tensor of magnetic permeability for the anisotropic media (including limited) conventional today it is ambiguously determined and it is not necessary), that considers frequency (temporary) and spatial dispersion, it is proposed to use kinematic inductance and capacity. In this case they seemingly are limited to isotropic media, speaking about the magnetic and dielectric constants. The authors for the completeness of account introduce the concept of the vector potential of electric field and refine the role of the vector potential of magnetic field in the equations of induction. It is shown what concept is, as the kinetic inductance of charges plays in the electrodynamics not less important role, than dielectric and magnetic constant. Is developed the mathematical model of the temporary dispersion of electromagnetic waves in the conductors and the dielectrics with the use of a physical quantity of dielectric constant, which does not depend on frequency. It is shown that in the confined plasma the transverse plasma resonance can exist. The concept of kinetic capacity is introduced. Is introduced the scalar- vector potential, in which the scalar potential of charge and its field they depend on speed. Within the framework the conversions of Galileo are obtained conversions pour on upon transfer of one inertial to another. The introduction of this potential made it possible within the framework the conversions of Galileo to explain the phase aberration and the transverse Doppler effect, and also power interaction of the current carrying systems without the use of a postulate about the Lorentz force. The results of the experimental studies, which showed, are given that the charge is not the invariant of speed.

The approach presented by the authors is convenient for the systems limited in the space, with the sizes, much more than the dimensions of the heterogeneity of field (wavelength of emission). They are borrowed from electrical engineering, and the authors compare their results with the experiment from elektromagnitostatik. The effects of spatial dispersion in this region are unessential, and the authors lawfully them disregard.

The book is intended for the specialists in the region of electrical engineering and electronics, radiophysics and radio engineering, technical protection of 
information. It is possible to recommend also to students and to graduate students, who specialize in the fields of science indicated.

I with the clean conscience recommend the book for the publication, although with some critical observations it is not agreeable. In connection with this he would want to the authors to give of the friendly observations, which they, if they want, can consider.

1. In my opinion the authors assign special theory of relativity greater than that, on what it pretends. Specifically, the Lorenz conversions in Poincare's account relate to the field theory, i.e., to the linear electrodynamics of the three-dimensional unlimited void (I even I do not mention word "vacuum"). Very equations of Maxwell, augmented by equations of motion of medium, can be Lorenz not invariant, although it is correct to describe the process being investigated.

2. Finally, the tensor of the dielectric constant, which considers spatial dispersion, strictly speaking, can be introduced for the unlimited in the space systems (sizes of which much more than the size of heterogeneity pour on). These are not observation, but the wish to the authors, if they will develop further their ideas and will consider spatial dispersion.

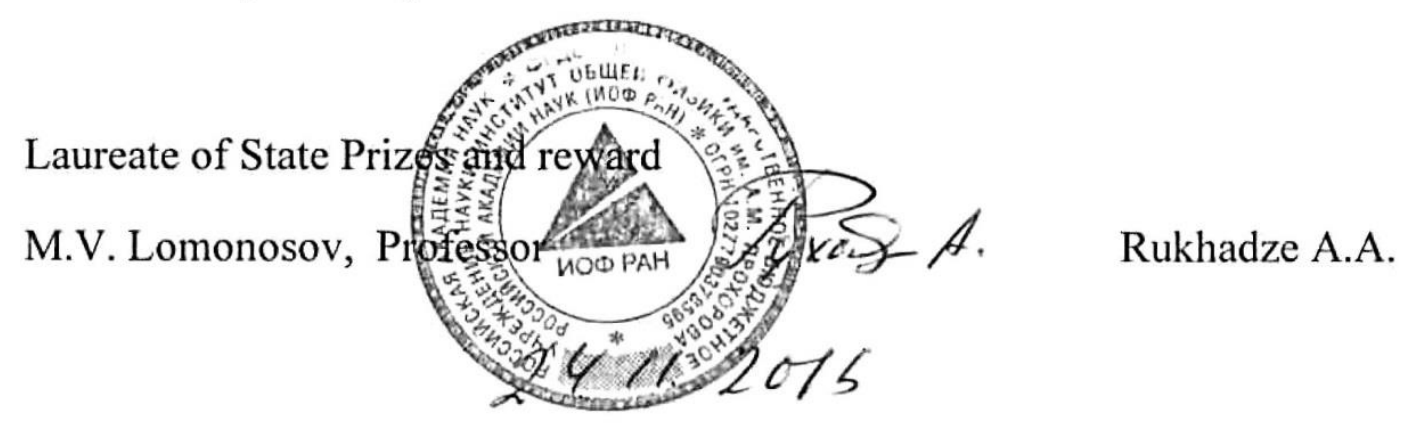




\title{
Review
}

\author{
to the monograph of Mende F. F. Dubrovin A. S. «Alternative ideology of \\ electrodynamics", 2016, 210 p., Fig. 62, ref. 78
}

At present classical electrodynamics consists of two not connected together parts. From one side this of Maxwell's equation, the describing wave processes, from other side - the Ampere law, which describes power interaction of the current carrying systems, and up to now there was no that component, which could combine these odd parts.

For the first time the laws of induction, from which follow Maxwell's equations, were recorded in his famous treatise, where was introduced the concept of bias current and the vector potential of magnetic field. But not all of physics know that those equations, which is conventionally designated as Maxwell's name, these are those equations, which are recorded in his famous treatise. His equations Maxwell wrote in the kvternion form use by the substantional derivative. Subsequently Hertz and Heaviside excluded from the substantional derivative its convective part, after writing down Maxwell's equations in the partial derivatives. A drawback in this approach is the impossibility of obtaining the conversion pour on upon transfer of one inertial system to another. One should, however, note that by Maxwell was used only one law of induction, which is conventionally designated as the law of the induction of Faraday, and who indicates how a change in the magnetic pour on it leads to the appearance pour on electrical. However, the symmetrical law of induction, which shows, how a change in the electrical pour on it leads to the appearance pour on magnetic, Maxwell so did not write down. However, if we follow Maxwell, and to write down these both equations of induction with the use by the substantional derivative, then it is possible to symmetrize these laws. The dependence of the scalar potential of charge on the speed follows from this symmetrization. This approach gives the possibility to introduce the concept of scalar- vector potential, and also transformation laws pour on upon transfer of one inertial system to another within the framework of conversions Galileo. These result should be considered most important in the criticized monograph. Further development of these ideas made possible to obtain the conversions indicated by matrix method, and also within the framework the trans-coordinate electrodynamics, whose basic ideas are also represented in the monograph. Moreover, this approach made it possible to connect the together odd parts of the electrodynamics and showed that the dependence of scalar potential on the speed is the basis of all laws of electrodynamics, charges connected with the motion. The symmetrization of the laws of induction made it possible to also introduce this new concept as kinetic capacity,

In the monograph the role of the vector potential of magnetic field in the equations of induction is refined and the concept of the vector potential of electric field is introduced. It is shown what concept is, as the kinetic inductance of charges plays in the electrodynamics not less important role, than dielectric and magnetic constant. Is developed the mathematical model of the dispersion of electromagnetic waves in the conductors and the dielectrics with the use of a physical quantity of dielectric constant, which does not depend on frequency. It is shown that in the confined plasma the transverse plasma resonance can exist. Provides a new way to display the wave equation. The results of the experimental studies, which showed, are given that the charge is not the invariant of speed. Monograph deserves highly estimation and can be recommend for the publication.

Chairman of department of the applied repentions of the radiophysics department of Kharkov national university V. N. Karazin, dof phiz.- ma Aciences, Professor

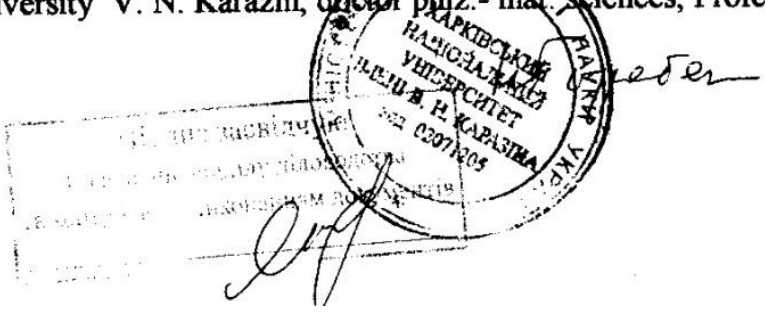

Gorobez N. N. 


\section{CONTENTS}

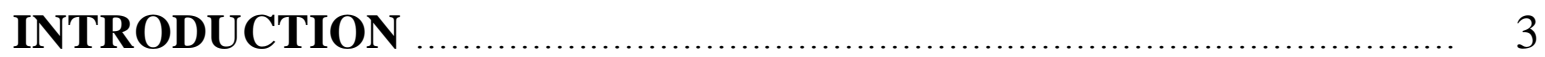

PART I

REFINEMENT OF THE CONCEPTS OF THE CLASSICAL ELECTRODYNAMICS

\section{CHAPTER 1}

THE LAWS OF INDUCTION AND THEIR ROLE

IN THE CLASSICAL ELECTRODYNAMICS …............................ 10

$\S 1$. Maxwell equations and Lorentz force ................................................... 10

$\S 2$. Laws of the magnetoelectric induction .................................................. 13

$\S 3$. Laws of the electromagnetic induction .............................................. 22

$\S 4$. Plurality of the forms of the writing of the electrodynamic laws ................ 24

\section{CHAPTER 2}

THE ROLE AND THE PLACE FOR THE KINETIC INDUCTANCE OF CHARGES IN THE CONTEMPORARY ELECTRODYNAMICS 26

$\S 5$. Who and as introduced the frequency dispersion of the dielectric constant

§ 6. Plasma-like media......................................................................... 27

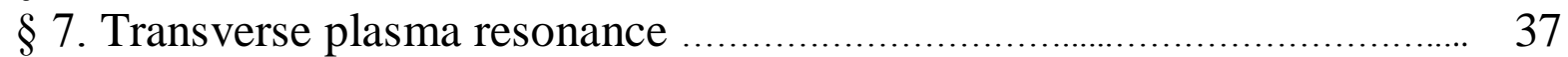

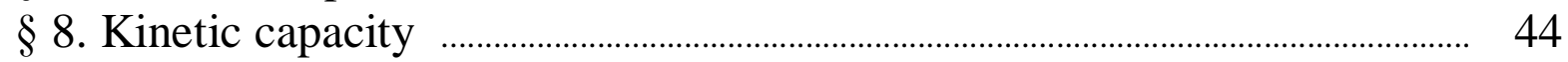

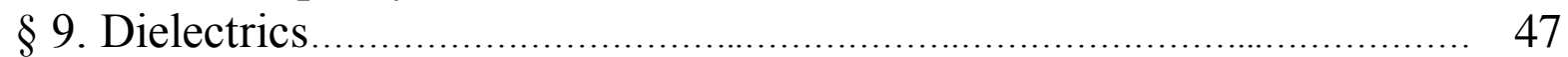

\section{CHAPTER 3}

NEW PROCEDURES AND CONCEPTS _........................................ 56

$\S 10$. Surface kinetic inductance ……....................................................... 56

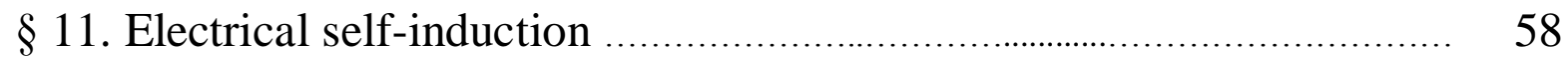

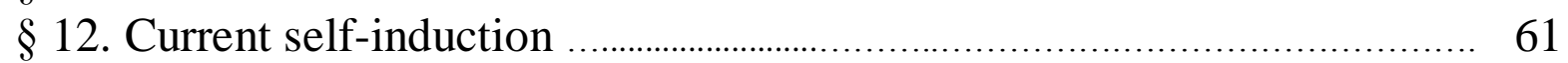

$\S 13$. New method of obtaining the wave equation, the potential

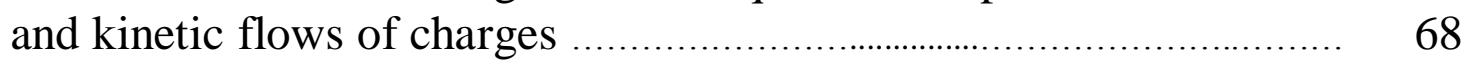

$\S 14$. Transient processes in the sections of the long lines ............................... 76 


\section{PART II}

\section{CHAPTER 4}

NEW APPROACHES AND DETERMINATIONS ……..................... 82

$\S 15$. Dynamic potentials and the field of the moving charges .................... 82

$\S 16$. The Vlasov equations and Lagrangian of the moving charge in the concept of the scalar-vector potential

$\S 17$. Trans-coordinate electrodynamics in time-spatial gipercontinuum and the Mende conversion ........................................................ 95

$\S 18$. Electric fields in the concept of the scalar-vector potential................. 113

$\S 19$. Power interaction of the current carrying systems, homopolar induction and the ponderomotive forces ...

$\S 20$. Experimental confirmation of the dependence of the scalar potential of charge on its relative speed

$\S 21$. Electric pulse of nuclear and other explosions

$\S 22$. Theoretical bases of giperkontinual physics and giperkontinual electrodynamics

$\S 23$. Special features of the mathematical apparatus for the classical electrodynamics

\section{PART III}

\section{OTHER NEW HYPOTHESES, EXPERIMENTAL RESULTS AND THE TECHNICAL SOLUTIONS}

\section{CHAPTER 5}

\section{OTHER HYPOTHESES, EXPERIMENTAL RESULTS AND THE}

\section{TECHNICAL SOLUTIONS}

$\S 25$. Is laser quantum generator?

$\S 26$. Physical substantiation of Huygens principle and the reciprocity theorem

$\S 27$. New system of units

$\S 28$. The liquid-drop model of electron and atom .

$\S 29$. Gravitational mass defect

$\S 30$. Three-dimensional coherence

$\S 31$. Ferroelectric transformer 
PACS 11.10.-Z

Mende F. F., Dubrovin A. S.

Alternative ideology of electrodynamics. M.: Пepo, 2016. - 216 p., Fig. 62, ref. 78. ISBN

Mende Fedor Fedorovich, Dubrovin Anatoliy Stanislavovich mende_fedor@mail.ru asd_kiziltash@mail.ru

\section{ALTERNATIVE IDEOLOGY OF ELECTRODYNAMICS}

